\title{
ACIS-I observations of NGC 2264. Membership and X-ray properties of PMS stars ${ }^{\star}$
}

\author{
E. Flaccomio, G. Micela, and S. Sciortino
}

\begin{abstract}
INAF - Osservatorio Astronomico di Palermo Giuseppe S. Vaiana, Palazzo dei Normanni, 90134 Palermo, Italy e-mail: [ettoref; giusi;sciorti]@astropa.inaf.it
\end{abstract}

Received 24 February 2006 / Accepted 5 April 2006

ABSTRACT

\begin{abstract}
Aims. This paper's goal is to improve the member census of the NGC 2264 star-forming region and study the origin of X-ray activity in young PMS stars.

Methods. We analyze a deep, $100 \mathrm{ks}$ long, Chandra ACIS observation covering a $17^{\prime} \times 17^{\prime}$ field in NGC 2264 . The preferential detection in X-rays of low-mass PMS stars gives strong indications of their membership. We study X-ray activity as a function of stellar and circumstellar characteristics by correlating the X-ray luminosities, temperatures, and absorptions with optical and nearinfrared data from the literature.

Results. We detect 420 X-ray point sources. Optical and NIR counterparts are found in the literature for $85 \%$ of the sources. We argue that more than $90 \%$ of these counterparts are NGC 2264 members, thereby significantly increasing the known low-mass cluster population by about 100 objects. Among the sources without counterpart, about $50 \%$ are probably associated with members, several of which we expect to be previously unknown protostellar objects. With regard to activity we confirm several previous findings: X-ray luminosity is related to stellar mass, although with a large scatter; $L_{\mathrm{X}} / L_{\mathrm{bol}}$ is close to, but almost invariably below, the saturation level, $10^{-3}$, especially when considering the quiescent X-ray emission. A comparison between CTTS and WTTS shows several differences: CTTS have, at any given mass, activity levels that are both lower and more scattered than WTTS; emission from CTTS may also be more time variable and is on average slightly harder than for WTTS. However, we find evidence in some CTTS of extremely cool, $\sim 0.1-0.2 \mathrm{keV}$, plasma which we speculate is heated by accretion shocks.

Conclusions. Activity in low-mass PMS stars, while generally similar to that of saturated MS stars, may be significantly affected by mass accretion in several ways: accretion is probably responsible for very soft X-ray emission directly produced in the accretion shock; it may reduce the average energy output of solar-like coronae, at the same time making them hotter and more dynamic. We briefly speculate on a physical scenario that can explain these observations.
\end{abstract}

Key words. stars: activity - stars: coronae - stars: pre-main sequence - open clusters and associations: individual: NGC $2264-$ X-rays: stars

\section{Introduction}

The collapse of molecular cores and the early evolution of premain sequence (PMS) stars+disk systems involve a variety of complex phenomena leading to the formation of main sequence (MS) stars and planetary systems. Most of these phenomena, and their influence on the outcome of the formation process, are not yet fully understood.

The X-ray observations of star-forming regions have proved an invaluable tool for star formation studies. On one hand, because of the much higher luminosity of PMS stars in the X-ray band with respect to older field stars, deep imaging observations are one of the few effective means of selecting unbiased samples of members comprising both classical T-Tauri stars (CTTS) and, most importantly, the otherwise hard to distinguish weak-line TTauri stars (WTTS). Selection of a complete member sample is of paramount importance for any star formation study, such as those focused on the initial mass function (Salpeter 1955), the star formation history (e.g. Palla \& Stahler 2000), the evolution of circumstellar disks and planetary systems (e.g. Haisch et al. 2005), and binarity (e.g. Lada 2006). On the other hand, the conspicuous X-ray activity of PMS stars is one of the aspects of

* Tables 1, 3, 4 and 6 are only available in electronic form at http://www.edpsciences.org the PMS stellar evolution that are not yet well understood, both with respect to its physical origin and to its consequences for the stellar/planetary formation process. Indeed, the ionization and heating caused by the penetrating X-ray emission might have a significant impact on the evolution of star/disk systems (Igea \& Glassgold 1999; Glassgold et al. 2004), as well as on that of the star forming cloud as a whole (Lorenzani \& Palla 2001).

The high X-ray activity levels of PMS stars (e.g. Preibisch et al. 2005) have often been attributed to a "scaled up" solarlike corona formed by active regions. This is the same picture proposed for MS stars, for which the X-ray activity is related to the stellar rotation (e.g. Pizzolato et al. 2003), evidence that a stellar dynamo is responsible for the creation and heating of coronae. For most non-accreting PMS stars (WTTS), the fractional X-ray luminosity, $L_{\mathrm{X}} / L_{\mathrm{bol}}$, is indeed close to the saturation level, $10^{-3}$, seen on rapidly rotating MS stars (Flaccomio et al. 2003b; Preibisch et al. 2005; Pizzolato et al. 2003). This might suggest a common physical mechanism for the emission of X-rays in WTTS and MS stars or, at least, for its saturation. However, the analogy with the Sun and MS stars may not be fully valid, because: i) the relation between activity and rotation is not observed in the PMS (Preibisch et al. 2005; Rebull et al. 2006); ii) with respect to the Sun at the maximum of its activity cycle, saturated WTTS have $L_{\mathrm{X}} / L_{\mathrm{bol}} \sim 1000$ times greater and plasma temperatures that are also significantly higher. 
The X-ray emission of CTTS, PMS stars that are still undergoing mass accretion, is even more puzzling. With their circumstellar disks and magnetically regulated matter inflows and outflows, CTTS are complex systems. With respect to their $\mathrm{X}$-ray activity, the bulk of the observational evidence points toward phenomena similar to those occurring on WTTS. However, CTTS have significantly lower and unsaturated values of $L_{\mathrm{X}}$ and $L_{\mathrm{X}} / L_{\text {bol }}$ (Damiani \& Micela 1995; Flaccomio et al. 2003a,b; Preibisch et al. 2005). In apparent contradiction to this latter result, high-resolution X-ray spectra of two observed CTTS, TW Hydrae (Kastner et al. 2002; Stelzer \& Schmitt 2004) and BP Tau (Schmitt et al. 2005), have indicated that soft X-rays may also be produced in accretion shocks at the base of magnetic funnels. Moreover, magnetic loops connecting the stellar surface with the inner parts of a circumstellar disk may produce some of the strongest and longer-lasting flares observed on PMS stars (Favata et al. 2005). The recent detection of X-ray rotational modulation (Flaccomio et al. 2005), however, implies that emitting structures are generally compact, so that these long loops cannot dominate the quiescent X-ray emission.

NGC 2264 is a 3 Myr-old star-forming region located at $\sim 760$ pc (Sung et al. 1997) in the Monoceros. Compared to the Orion Nebula Cluster (ONC) and Taurus, NGC 2264 has intermediate stellar density and total population, making it an interesting target for investigating the dependence of star formation on the environment. It is on average older than the ONC $(\sim 1 \mathrm{Myr})$, but star formation is still active inside the molecular cloud in at least two sites where a number of protostars and prestellar clumps have been detected (Young et al. 2006; Peretto et al. 2006). It is therefore a useful target for the study of the formation and time evolution of young stars. Its study is eased by the presence of an optically thick background cloud, effectively obscuring unrelated background objects, and by the low and uniform extinction of the foreground population (Walker 1956; Rebull et al. 2002). Despite being the first star forming region ever identified as such, the low-mass population of NGC 2264 is still not well characterized: proper motion studies (Vasilevskis et al. 1965) have been restricted to high mass objects; several studies have identified the CTTS population using disk and accretion indicators (Park et al. 2000; Rebull et al. 2002; Lamm et al. 2004), but have missed WTTS; past X-ray observations with ROSAT (Flaccomio et al. 2000) have been useful in identifying the WTTS population, but have not been sensitive enough to detect low-mass $\left(M \leq 0.3 M_{\odot}\right)$ and embedded stars. We present here results from the analysis of a deep Chandra observation of the region. Another similar observation of a region just to the north of the one considered here has been analyzed by Ramírez et al. (2004a). The X-ray properties of NGC 2264 members derived in the present paper and by Ramírez et al. (2004a), augmented with similar data for the Orion Flaking Fields (Ramírez et al. 2004b) are studied by Rebull et al. (2006) in comparison with the results of the COUP survey (Getman et al. 2005). Results from the same Chandra observation analyzed here on the peculiar binary system KH 15D have been presented by Herbst \& Moran (2005). Finally, the properties of three embedded X-ray sources near Allen's source, observed with XMM-Newton, have been recently presented by Simon \& Dahm (2005).

The paper is organized as follows. We begin (Sect. 2) with the presentation of the X-ray data, its reduction, source detection, and photon extraction. In Sect. 3 we then introduce the optical and near infrared data used to complement the X-ray observation. In Sect. 4 we present the temporal and spectral analysis of X-ray sources and derive X-ray luminosities. Sections 5 and 6

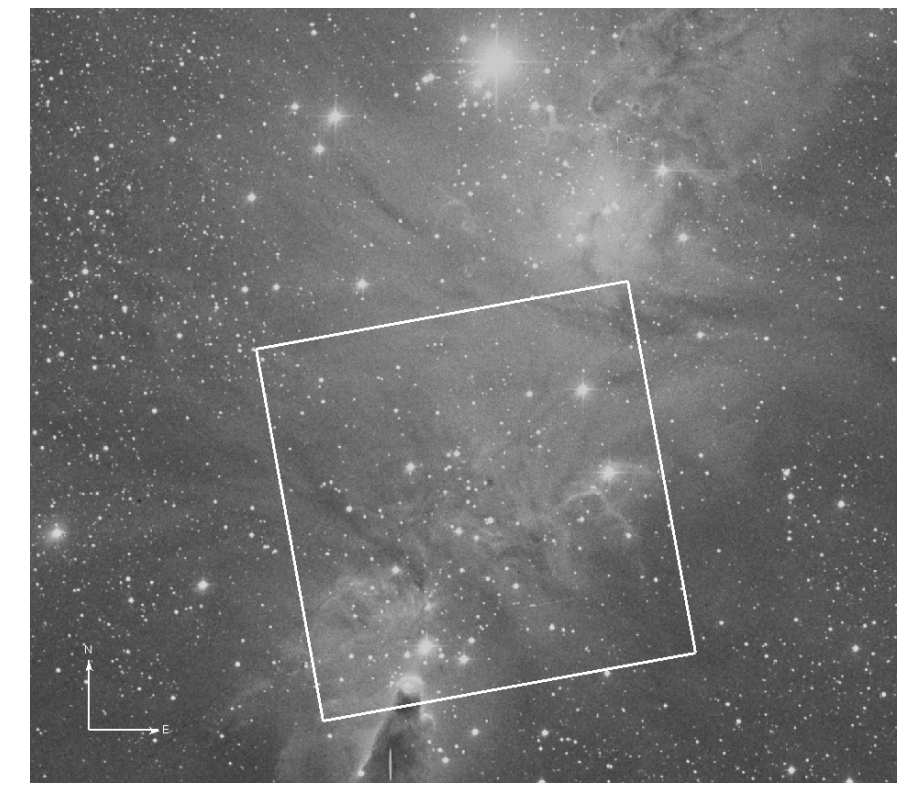

Fig. 1. Digitized Sky Survey image of NGC 2264. The field of view of the Chandra-ACIS observation discussed in this paper is shown as a white square. The famous Cone Nebula is visible toward the bottom of the image and the $\mathrm{O} 7 \mathrm{star} \mathrm{S}$ Mon is close to the upper edge.

then discuss our results with respect to cluster membership and the origin of X-ray activity on PMS stars. We finally summarize and draw our conclusions in Sect. 7.

\section{X-ray data and preparatory analysis}

We obtained a $97 \mathrm{ks}$ long ACIS-I exposure of NGC 2264 on 28 Oct. 2002 (Obs. Id. 2540; GO proposal PI S. Sciortino). The $17^{\prime} \times 17^{\prime}$ field of view (FOV) of ACIS is shown in Fig. 1, superimposed on the Digitized Sky Survey image of the region. It was centered on RA $6^{\mathrm{h}} 40^{\mathrm{m}} 58^{\mathrm{s}} .7$, Dec $9^{\circ} 34^{\prime} 14^{\prime \prime}$ (roll angle: $79^{\circ}$ ). Figure 2 shows a color rendition of the spatial and spectral information we obtained. ACIS was operated in FAINT mode with CCD 0, 1, 2, 3, 6, and 7 turned on. Data obtained with CCD 6 and 7, part of the ACIS-S array, will not be discussed in the following because of the very degraded point-spread function (PSF) and effective area resulting from their large distance from the optical axis.

\subsection{Data preparation}

Data reduction, starting from the level 1 event file, was performed in a standard fashion, using the CIAO 2.3 package and following the threads provided by the Chandra X-ray Center ${ }^{1}$. Several IDL custom programs were also employed. First, we corrected the degradation in the spectral response due to the charge transfer inefficiency (CTI), which occurred in particular during the first months of the Chandra mission, using the ACIS_PROCESS_EVENTS CIAO task. We then produced a level 2 event file by retaining only events with grade $=0,2,3,4,6$ and status $=0$. Finally we corrected the data for the time dependence of the energy gain using the CORR_TGAIN utility.

The X-ray stellar sources have, on average, a different spectrum with respect to the ACIS background. The total signalto-noise ratio (SNR) of sources can therefore be maximized by filtering out events with energy outside a suitable spectral

\footnotetext{
${ }^{1}$ See cxc.harvard.edu
} 


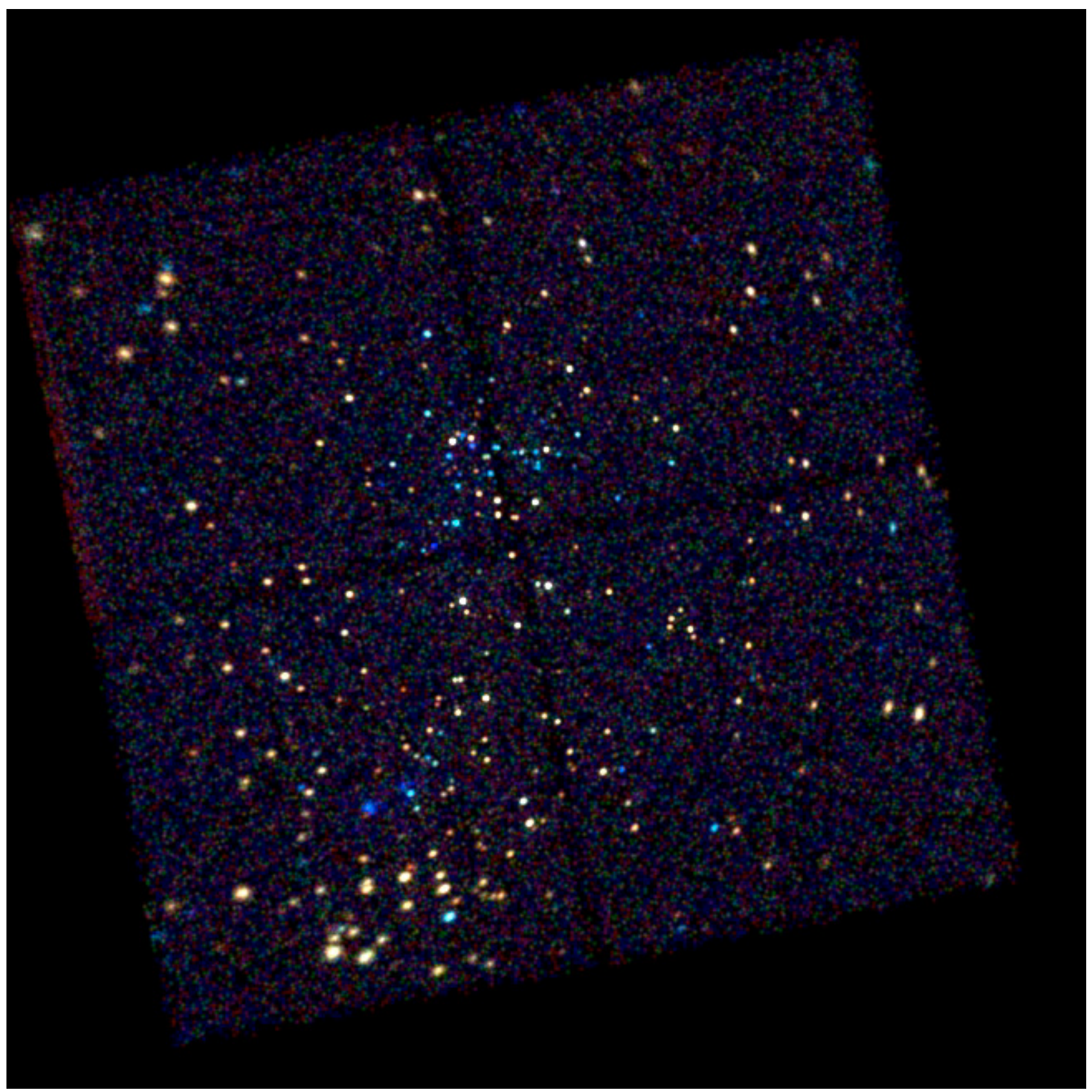

Fig. 2. NGC 2264 as seen in X-rays by ACIS. The true color (RGB) image is constructed from images in three energy bands: [200:1150] eV (red), [1150:1900] (green), and [1900:7000] (blue). Red therefore indicates soft and unabsorbed sources, blue hard and/or absorbed sources.

band: we first performed a preliminary source detection as discussed in Sect. 2.2 on the whole event list. We then defined a radius, $R_{97}$, for each source such that $97 \%$ of the PSF counts fall within this radius (Sect. 2.3). We extracted source photons for all sources from circles with $R=0.5 \times R_{97}$ and background photons from a single background region that excludes photons from all sources within their respective $R_{97}$ (a sort of "Swiss cheese" image with the sources carved out). We then computed the total SNR of sources ${ }^{2}$ for a fine grid of minimum and maximum energy cuts. The highest source SNR was obtained for

${ }^{2} S N R_{\mathrm{tot}}=\sum\left(S^{i}-\alpha_{i} B\right) / \sqrt{\sum\left(S^{i}+\alpha_{i} B\right)}$ where $\alpha_{i}=A_{s}^{i} / A_{b} ; S_{i}$ and $A_{s}^{i}$ are the number of counts in the $i$ th source region and its area, respectively. $B$ and $A_{b}$ are the background counts and relative extraction area, respectively. The background was taken for the present purpose as uniform within the FOV.
$E_{\min }=200 \mathrm{eV}$ and $E_{\max }=7000 \mathrm{eV}$. With these cuts, the number of photons in the source extraction area (including background photons) is reduced to $96 \%$ of the total, while the background is reduced to $28 \%$ of the total. We checked that consistent results are obtained by maximizing the SNR of faint sources only ( $<20$ net counts), which may have a different average spectrum and are the ones we are most interested in for the purpose of detection.

After filtering in energy, the time-integrated background is 0.07 counts per $\operatorname{arcsec}^{2}$, consistent with nominal values. The background was constant in time except for a small flare with a peak reaching about twice the quiescent rate. The flare starts $13 \mathrm{ks}$ after the beginning of the observation and lasts $\sim 1 \mathrm{ks}$. The effect of the background flare on source light curves is negligible however, even for faint sources, i.e. those most affected by the background. This is confirmed by the negative results of 
Kolmogorov-Smirnov variability tests (see Sect. 4.1) performed on the background extraction regions relative to each source. In the study of source lightcurves (Sect. 4.1), we will therefore assume a constant background.

\subsection{Source detection}

We detected sources using the PWDetect code (Damiani et al. $1997)^{3}$. The significance threshold was set to $4.6 \sigma$. According to extensive simulations of source-free fields with the background level of our observation, this threshold corresponds to an expectancy of 10 spurious sources in the whole FOV. PWDetect reports 423 sources. Upon careful inspection we removed three entries relative to sources that were detected twice, leaving a total of 420 distinct sources. Twenty eight of these are below the $5.0 \sigma$ significance threshold, corresponding to the more conservative criteria of one expected spurious source in the FOV. Background-subtracted source counts in the $0.2-7 \mathrm{keV}$ band are derived by PWDetect directly from the wavelet transform of the data. Effective exposure times at the source positions, averaged over the PSF, are also computed by PWDetect from an exposure map created with standard CIAO tools assuming an input energy of $2.0 \mathrm{keV}^{4}$. Detected sources are listed in Table 1. In the first eight columns we report source number, sky positions with uncertainty, distance from the Chandra optical axis, source net counts (in the $0.2-7 \mathrm{keV}$ band), effective exposure time, and the statistical significance of the detection.

\subsection{Photon extraction}

Source and background photon extraction for spectral and timing analysis was performed using CIAO and custom IDL software. We first determined the expected PSF for each source using the CIAO MKPSF tool, assuming a monochromatic source spectrum $(E=1.5 \mathrm{keV})$. We thus determined the expected encircled energy fraction as a function of distance from the source. Photons' extraction circles were defined so as to contain $90 \%$ of the PSF, save for 28 sources for which the encircled PSF fractions were reduced to values ranging from $74 \%$ to $89 \%$ to avoid overlap with neighboring sources. The local background for each source was determined from annuli whose inner radii exclude $97 \%$ of the PSF and whose outer radii are twice as large. In order to eliminate contamination of the background regions due to the emission of neighboring sources, we excluded from these annuli all the intersections with the $97 \%$ encircled counts circles of other sources. The area of background extraction regions were computed through a mask in which we drilled regions outside the detector boundaries and circles containing $97 \%$ of the PSF photons from all sources.

For sources with at least 50 photons, source and background spectra suited to the XSPEC spectral fitting package were then created using standard CIAO tools. Corresponding response matrices and effective areas (RMF and ARF files, respectively) were also produced with CIAO. For spectral analysis, spectra were energy binned so that each bin contains a fixed number of photons, depending on net source counts $N_{\text {src }}$ : 15 photons per bin for $N_{\text {src }}>200,10$ photons for $100<N_{\text {src }}<200$, and

\footnotetext{
3 Available at:

http://wwW . astropa.unipa.it/progetti_ricerca/PWDetect

4 The choice of energy is not crucial: the ratio between the effective area at the source position and on the optical axis, which is the quantity we use to define the effective exposure time, has only a small dependence on energy.
}

5 photons for $N_{\text {src }}<100$. Because spectral analysis (Sect. 4.2) was restricted to energies $>0.5 \mathrm{keV}$, the first energy bin was forced to begin at that energy.

\section{Ancillary data}

In this section we discuss the non-X-ray data that we collected from the literature on the known objects in the FOV of our ACIS observation, and in particular on the detected X-ray sources. We first describe the cross-identifications with spatially complete optical, NIR, and X-ray catalogs (Sect. 3.1) and the informations we collected and/or derived from these catalogs (Sect. 3.2). We then briefly discuss the identifications of our X-ray sources with spatially incomplete mid-IR and millimiter catalogs (Sect. 3.3).

\subsection{Cross-identifications - optical/NIR/X-ray catalogs}

We cross-identified our X-ray source list with catalogs from the following optical/NIR surveys covering the whole area of our ACIS field: 2Mass (NIR photometry), Walker (1956, optical photometry + spectroscopy), Rebull et al. (2002, optical/NIR photometry + low resolution optical spectroscopy $)^{5}$, Lamm et al. (2004, optical photometry + variability), Dahm \& Simon (2005, optical photometry + spectroscopy), Flaccomio et al. (2000, $\mathrm{X}$-ray sources). The seven cross-identified catalogs are listed in the first column of Table 2. In the 2nd column, we indicate the number of objects within the ACIS FOV and, in the third, the number of objects identified with ACIS sources and of those for which the identification is unique. Adopted positional tolerances for cross-identifications are reported in the 4th column, either as a single figure for the whole catalog or as a range when defined for each individual object (see the table footnotes). They are based on the uncertainties quoted for each catalog.

Among the catalogs considered here, the deepest photometric surveys are 2Mass in the NIR $\left(\mathrm{JHKs}^{6}\right)$ and Lamm et al. (2004) in the optical $\left(V R_{\mathrm{c}} I_{\mathrm{c}}\right)$. A comparison with the isochrones of Siess et al. (2000, hereafter SDF) in the optical and NIR colormagnitude diagrams (Figs. 3 and 4) indicates that at the distance of NGC 2264, 2Mass reaches down to about $0.1 M_{\odot}$ for $10 \mathrm{Myr}$ old stars, i.e. the oldest expected in the region, while Lamm et al. (2004) reaches slightly deeper for unabsorbed stars, but is obviously less sensitive to highly absorbed ones. Within the ACIS FOV, spectral types are given for 7 stars by Walker (1956), for 87 stars by Rebull et al. (2002), for 150 stars by Lamm et al. (2004), and for 157 stars by Dahm \& Simon (2005).

We created a master list of cross-identified objects following a step-by-step procedure. In the first step we matched ACIS sources with 2Mass objects: first we registered the ACIS coordinates to the 2 Mass ones by iteratively cross-identifying the two catalogs and shifting the ACIS coordinates by the mean offset of uniquely identified source pairs. Identification radii were chosen as the quadrature sum of the position tolerances defined above (Table 2). We then created a joint catalog of objects containing all matched and unmatched ACIS and 2Mass objects, assigning them coordinates from 2MASS when available. In the following step we repeated the above process using the ACIS+2Mass cata$\log$ as reference and matching it with the Rebull et al. (2002) one. We then repeated the process with the Flaccomio et al. (2000),

\footnotetext{
5 The Rebull et al. (2002), catalog was updated following private communication from L. Rebull.

6 Note that in the following we neglect the small differences among NIR photometric systems and, in particular, that between the 2MASS $K$ s and the standard $K$ bands.
} 
Table 2. Catalogs used for cross-identification

\begin{tabular}{lrrcccc}
\hline \hline Catalog & $N_{\text {obj }}$ & $N_{\text {det }} / N_{\text {unique }}$ & $\begin{array}{c}\text { Id.Rad. } \\
{\left[{ }^{\prime \prime}\right]}\end{array}$ & $\begin{array}{c}\Delta \text { RA } \\
{\left[{ }^{\prime \prime}\right]}\end{array}$ & $\begin{array}{c}\Delta \text { Dec } \\
{\left[{ }^{\prime \prime}\right]}\end{array}$ & $\begin{array}{c}\text { Off }_{50 \%} \\
{\left[{ }^{\prime \prime}\right]}\end{array}$ \\
\hline ACIS & 420 & - & $1.0-12.6^{a}$ & -0.09 & -0.09 & 0.24 \\
2Mass & 1098 & $346 / 344$ & $0.5-1.4^{b}$ & 0.00 & 0.00 & - \\
Walker (1956) & 67 & $52 / 52$ & 1.0 & -0.45 & 0.08 & 0.47 \\
Rebull et al. (2002) & 511 & $236 / 235$ & 1.5 & 0.73 & -0.03 & 0.73 \\
Lamm et al. (2004) & 1598 & $305 / 299$ & 1.0 & -0.13 & 0.45 & 0.47 \\
Dahm \& Simon (2005) & 229 & $183 / 183$ & 1.0 & -0.16 & 0.18 & 0.36 \\
Flaccomio et al. (2000) & 84 & $80 / 80$ & $8.1-42.8^{c}$ & -0.85 & 1.49 & 3.70 \\
\hline
\end{tabular}

${ }^{a} \max \left[2 \sigma_{r d}, 1^{\prime \prime}\right]$. Mean/median: $1.74 " / 1.25^{\prime \prime}$.

${ }^{b} \max \left[2\left(\sigma_{\mathrm{RA}}^{2}+\sigma_{\mathrm{Dec}}^{2}\right)^{1 / 2}, 0.5^{\prime \prime}\right]$. Mean/median: $0.54^{\prime \prime} / 0.50^{\prime \prime}$.

$c 85 \%$ point source encircled energy radii (Flaccomio et al. 2000). Mean/median: 12.0"/9.2"

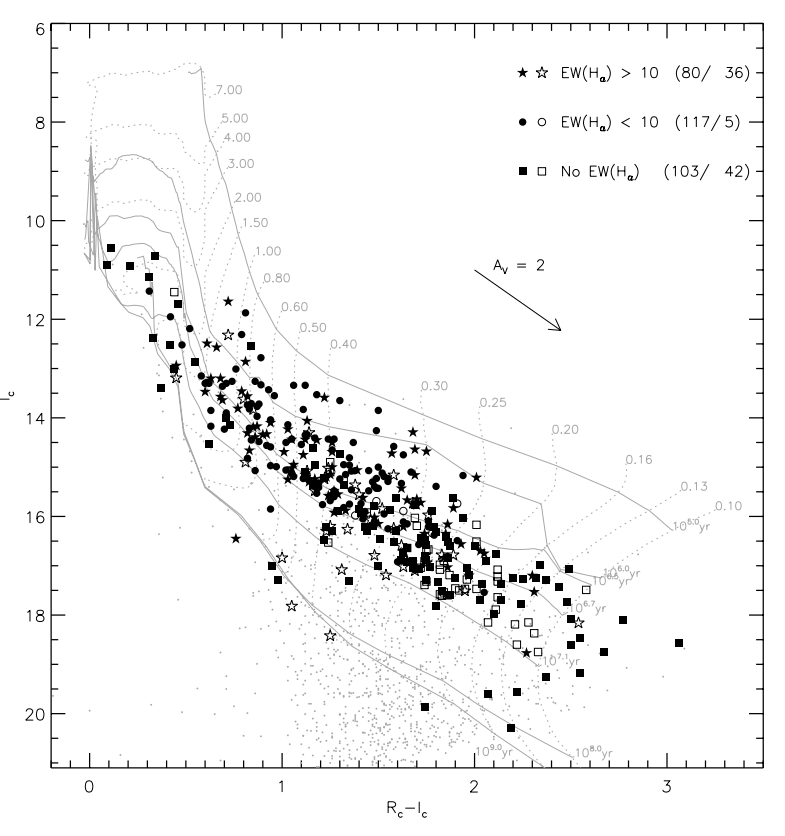

Fig. 3. Optical color-magnitude diagram of all the objects in the ACIS field of view. Larger symbols indicate X-ray sources or likely NGC 2264 members as defined in Sect. 3.2. Filled symbols refer to ACIS detected objects. As indicated in the legend, when possible we distinguish between CTTS and WTTS as defined by the EW of their $\mathrm{H}_{\alpha}$ line. The number of detected and undetected objects in each class is indicated in the legend. The grid shows the SDF evolutionary tracks and isochrones transformed to colors and magnitudes using the conversion table in Kenyon \& Hartmann (1995).

X-ray source list, then with the Lamm et al. (2004), Dahm \& Simon (2005), and Walker (1956) catalogs. The coordinate shifts of all catalogs with respect to the 2Mass system are given in Table 2 (Cols. 5 and 6), along with the median offsets between object positions and the reference 2 Mass catalog. After each step, identified source pairs and unidentified objects were checked individually and a small fraction of the identifications $(<1 \%)$ were modified. In the first step, for example, five identifications between four ACIS and five 2Mass sources were added: in two cases (sources \#64 and \#404), 2Mass sources were only slightly more distant with respect to the identification radii. In another case, the X-ray source, \#102, was situated at the edge of the detector, and its position was therefore more uncertain than the formal error indicated. In the last case the X-ray source, \#237, was detected between two nearby 2Mass objects and an identification was forced with both.

Table 3 lists, for the 1888 distinct objects in the ACIS FOV, consolidated coordinates and cross-identifications numbers for

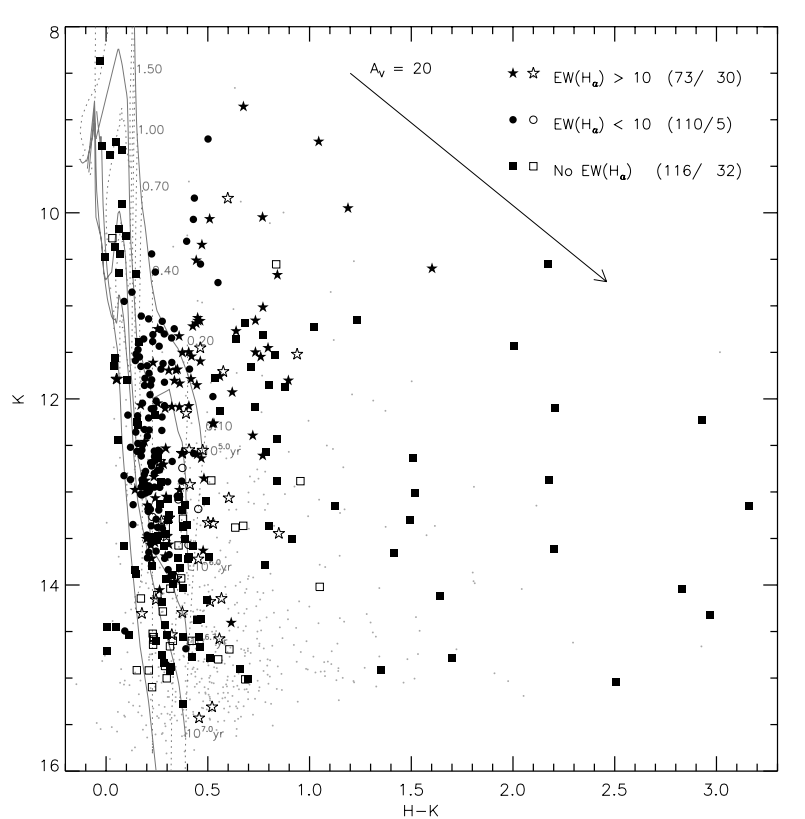

Fig. 4. Near-IR color magnitude diagram for the objects in the ACIS FOV. Symbols and tracks as in Fig. 3.

each of the seven catalogs. 425 rows refer to objects related to one of the 420 ACIS sources: 351 are identified with a single optical/NIR counterpart, two are identified with 5 and 2 counterparts respectively, and 67 do not have any optical or nearinfrared identification. The other 1463 rows in Table 3 refer to non ACIS-detected objects. For these latter we computed upper limits to the ACIS count rate using PWDetect and the same event file used for source detection. Measured count rates, repeated from Table 1, and upper limits are reported in Col. 12 of Table 3.

Focusing on the identification of ACIS sources with optical/NIR catalogs, given the relatively large number objects in the field of view, we can wonder how many of the identifications are due to chance alignment and not to a true physical association. We can constrain the number of chance identifications by assuming that positions in the two lists are fully uncorrelated. Because this is definitively not the case for our full X-ray source catalog, our estimate can only be considered a loose upper limit. Furthermore, limiting our X-ray sample to the 28 sources with significance below $5.0 \sigma, 9$ of which are expected to be spurious and whose positions will indeed be random, we can place an upper limit on the fraction of spurious sources associated with an optical object. This value is of interest when studying the X-ray 
properties of optically/NIR selected samples. In order to estimate the number of spurious identifications assuming uncorrelated positions, we proceeded as follows: for each X-ray source we considered optical objects within a circular neighborhood of area $A_{\text {nei }}$, within which source density is assumed uniform. We then estimated the fraction of $A_{\text {nei }}$ covered by identification circles. The sum of these fractions is our upper limit to the number of chance identifications. We repeated the calculation for radii of the neighborhood circle from $1.0^{\prime}$ to $4.0^{\prime}$. The upper limit to the number of chance identifications for the full sample of ACIS sources ranges from 13 to 15 . For the 28 source with significance $<5.0 \sigma$, we instead estimated no more than 2.5 chance identifications. If we assume that, out of the 28 sources, only the nine spurious detections have positions that are indeed uncorrelated with optical objects, we can scale this result and conclude that $\sim 1$ spurious X-ray source will be identified by chance with an optical object. Conversely, we can most likely locate spurious sources among the 15 sources whose detection significance is below $5.0 \sigma$ and that are not identified with optical/NIR catalogs.

\subsection{Characterization of stars in the FOV}

We collected optical and NIR data from the literature for all the stars in the master catalog assembled in the previous section. In Table 4 we report photometry and spectral types for our X-ray sources with unique optical identification. A total of $300 \mathrm{X}$-ray detected stars were assigned both $R_{\mathrm{c}}$ and $I_{\mathrm{c}}$ magnitudes and are plotted in Fig. 3 as filled symbols; 299 have $H$ and $K$ magnitudes and are plotted in Fig. 4 (264 of these also appear in Fig. 3). In both color-magnitude diagrams (CMDs), we also show SDF tracks and isochrones for reference, transformed to colors and magnitudes using the conversion table given by Kenyon \& Hartmann (1995) and shifted along the reddening vectors by the median extinction of known members $\left(A_{\mathrm{V}}=0.44\right)$ and vertically by the distance modulus corresponding to the adopted distance. The extinction law was adopted from Rieke \& Lebofsky (1985). For stars with spectral types, we derived effective temperatures, $T_{\text {eff }}$, bolometric corrections, $B C_{I}$, and intrinsic colors, $(R-I)_{0}$, using the relations compiled by Kenyon \& Hartmann (1995) and, for the temperatures of $\mathrm{M}$ stars, the intermediate-gravity scale of Luhman (1999). Using the available $R_{\mathrm{c}}$ and $I_{\mathrm{c}}$ photometry, we then derived extinction values $\left(A_{\mathrm{V}}=4.46 \times E(R-I)\right.$, where $\left.E(R-I)=(R-I)_{0}-(R-I)\right)$ and bolometric luminosities $\left(L_{\mathrm{bol}}=-0.4 \times\left[I_{\mathrm{c}}-B C_{I}-A_{\mathrm{V}} / 1.63-D M(760 \mathrm{pc})\right]\right)$. Finally we estimated masses and ages from the theoretical HR diagram, Fig. 5, through interpolation of the SDF evolutionary tracks. In summary, out of the $351 \mathrm{X}$-ray sources with a unique optical/NIR identification, we estimated $T_{\text {eff }}$ for $165 \mathrm{X}$-ray sources, $A_{\mathrm{V}}$ and $L_{\mathrm{bol}}$ for 163 , masses and ages for 161 .

Other than the sample of X-ray detected stars, that are likely cluster members, as discussed in Sect. 5, we also consider another sample of 83 X-ray undetected likely cluster members. These, plotted with empty symbols in Figs. 3-5, were chosen according to their position in the $I$ vs. $R-I$ diagram, the strength of the $\mathrm{H}_{\alpha}$ line (measured either spectroscopically or photometrically) and optical variability: first, we defined a cluster locus in the $I$ vs. $R-I$ diagram using the SDF tracks and the observed concentration of X-ray sources. The cluster locus was defined as the area either above the $10^{7.1} \mathrm{Myr}$ isochrone or to the left of the $0.8 M_{\odot}$ evolutionary track ${ }^{7}$. We then considered the following as

\footnotetext{
7 This latter condition was required by the convergence of the isochrones at higher masses and by the observed spread of stars in the "cluster locus", at least in part due to uncertainties.
}

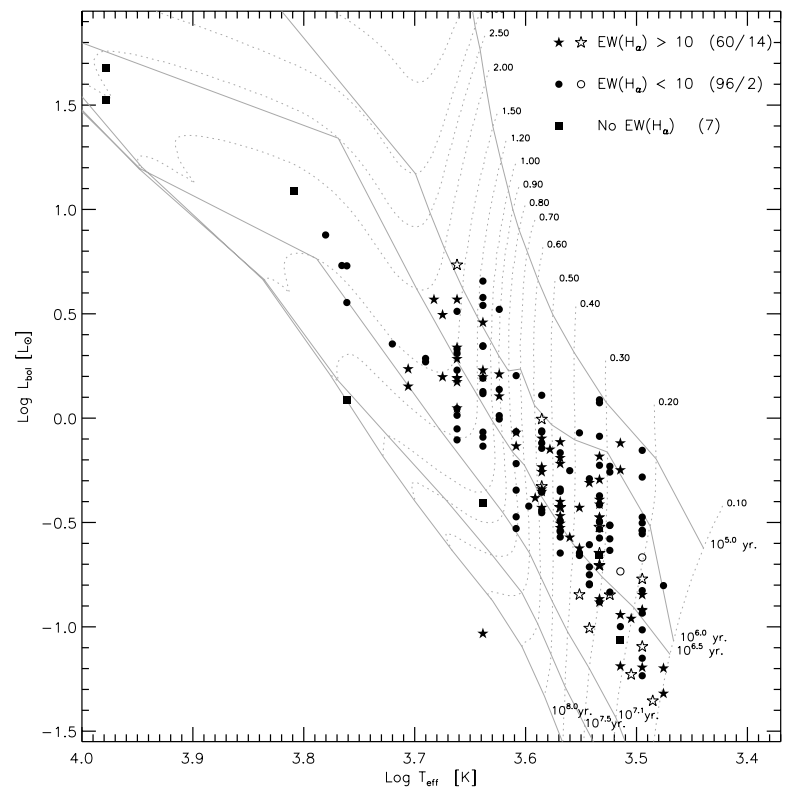

Fig. 5. Theoretical HR diagram for the subsample of likely members that could be placed in this diagram through optical photometry and spectral types. Symbols as in Fig. 3. Tracks and isochrones are by SDF.

likely members: (i) stars in the cluster locus and with strong $\mathrm{H}_{\alpha}$ emission according to the narrow-band $\mathrm{H}_{\alpha}$ photometry of Lamm et al. (2004), using the same criterion discussed by these authors; (ii) stars in the cluster locus with moderate (chromospheric) $\mathrm{H}_{\alpha}$ emission and with variable optical lightcurves (both periodic and irregular), again as discussed by Lamm et al. (2004); (iii) stars for which spectroscopic observations of the $\mathrm{H}_{\alpha}$ line were available and for which the measured EW was larger than 10 (the canonical CTTS threshold). In this sample of 83 X-ray undetected likely members, we were able to determine masses and ages for 16 stars.

Knowledge of the extinction is fundamental for deriving intrinsic X-ray luminosities of detected stars or upper limits for undetected ones (Sect. 4.3). For stars with no optical spectral type, $A_{\mathrm{V}}$ cannot be determined as indicated above. We therefore estimated extinctions from the NIR $J-H$ vs. $H-K$ diagram by dereddening stars that could be placed in this diagram onto the expected intrinsic locus. This was taken from Kenyon \& Hartmann (1995) for the stellar contribution and supplemented with the CTTS locus of Meyer et al. (1997). The degeneracy in the reddening solutions was solved by always taking the first intercept between the intrinsic locus and the reddening vector. Stars whose position was within $1.5 \sigma$ of the loci were assigned zero extinction. In summary, we obtained a total of 278 and 62 extinction values for X-ray detected stars and undetected likely members, respectively. These number exceed the number of extinctions derived from optical data by 115 and 46 for the same samples. Although these NIR extinction values are arguably more uncertain than the ones derived from spectral types, they are especially valuable for highly extinct objects.

\subsection{Cross-identifications - MIR/mm catalogs}

In addition to the wide field surveys described in Sect. 3.1, we also correlated our X-ray sources with three mid-infrared (MIR) and millimeter $(\mathrm{mm})$ catalogs recently published for the IRS 2 and IRS 1 regions: Young et al. (2006, hereafter Y06), Teixeira et al. (2006, T06), and Peretto et al. (2006, P06). These three 
surveys target very young and/or embedded objects and are therefore ideal for checking the nature of X-ray sources with no optical/NIR counterpart. They are, however, limited in area coverage: T06 lists Class I/0 sources detected with SPITZER in $\mathrm{a} \sim 3 \times 3 \operatorname{arcmin}^{2}$ region close to IRS 2, Y06 lists all SPITZER detected objects in a dense but even smaller $\sim 2 \times 2 \operatorname{arcmin}^{2}$ region slightly south-east of IRS 2, P06 list the pre/protostellar cores detected at $1.2 \mathrm{~mm}$ in both the IRS 1 and IRS 2 regions.

Within the area of Y06, the only MIR work available so far that lists all the objects detected in the surveyed region, we find MIR counterparts for 2 out of 4 X-ray sources lacking optical/NIR counterparts. In total, 4 of the 67 unidentified X-ray sources were assigned new counterparts: \#145 was associated with source 12 of T06, a Class I/0 source; \#228 was associated with the D-MM $15 \mathrm{~mm}$ core, indicated by P06 as probably starless; \#244 was associated with source 43 in Y06 and is, judging from its spectral energy distribution (SED), an absorbed class II/III PMS star that is relatively bright in $K$-band $(K=12.15$, Y06) ${ }^{8}$; finally, \#274 was associated with the D-MM $10 \mathrm{~mm}$ core, indicated as starless by P06, with source 1656 in Y06 and source 15 in T06 (characterized by a steeply rising MIR SED).

Among the X-ray sources with optical/NIR counterparts, 17 in the region covered by Y06 were identified with SPITZER sources, of which one, \#281, is a likely Class I source (Y06's source 62). Three more X-ray sources were identified with Class I/0 sources in the list of T06: \#150 and \#242 also having NIR counterparts, and \#194 (see also Sect. 6.3) with both NIR and optical $(I=19.89)$ counterpart. As for the P06 mm-cores, identifications are made uncertain by the limited spatial resolution of the $\mathrm{mm}$ data (cf. P06). The core D-MM 14, classified as protostellar by P06, is probably associated with ACIS source \#89 (offset: $\left.1.2^{\prime \prime}\right)$, an optically faint $(V=20.91)$ and NIR bright $(K=10.6)$ star with intense $\mathrm{H}_{\alpha}$ emission: $E W\left(\mathrm{H}_{\alpha}\right)=458$. Quite similarly, C-MM 1, also indicated as a protostellar core by P06, is offset by $1.3^{\prime \prime}$ from the ACIS source \#361, an optically visible source with strong $\mathrm{H}_{\alpha}\left(V=18.55, K=11.85, E W\left(\mathrm{H}_{\alpha}\right)=231\right)^{9}$ and it is therefore likely to be associated with it. Finally, C-MM 5, classified by P06 as a protostellar core, might be associated with ACIS \# 305 (offset: 1.9"). This ACIS source is in turn most likely associated with IRS 1 (offset: 0.8", Schreyer et al. 2003). Note, however, that P06 suggests that C-MM 5 may not be associated with IRS 1. A more detailed analysis of the X-ray properties of young protostars in NGC 2264 will be the subject of a future paper.

\section{Analysis}

In this section we analyze the X-ray properties of our ACIS sources as indicated by their ACIS lightcurves (Sect. 4.1) and spectra (Sect. 4.2). The results of the spectral analysis are then used in Sect. 4.3 to estimate X-ray luminosities.

\subsection{Temporal analysis}

Source variability was characterized through the KolmogorovSmirnov test. Column 9 of Table 1 reports the resulting probability that the distribution of photon arrival times is not compatible with a constant count-rate. Given the sample size (420 sources), a value below $0.1 \%$ (obtained for 72 sources)

\footnotetext{
8 Note that the source is missed by 2MASS because, at the 2MASS spatial resolution, it is blended with a nearby source.

9 Although Y06 report that C-MM 1 has no NIR counterpart, we associate it with 2MASS source 06411792+0929011 (offset $\left.=1.5^{\prime \prime}\right)$.
}

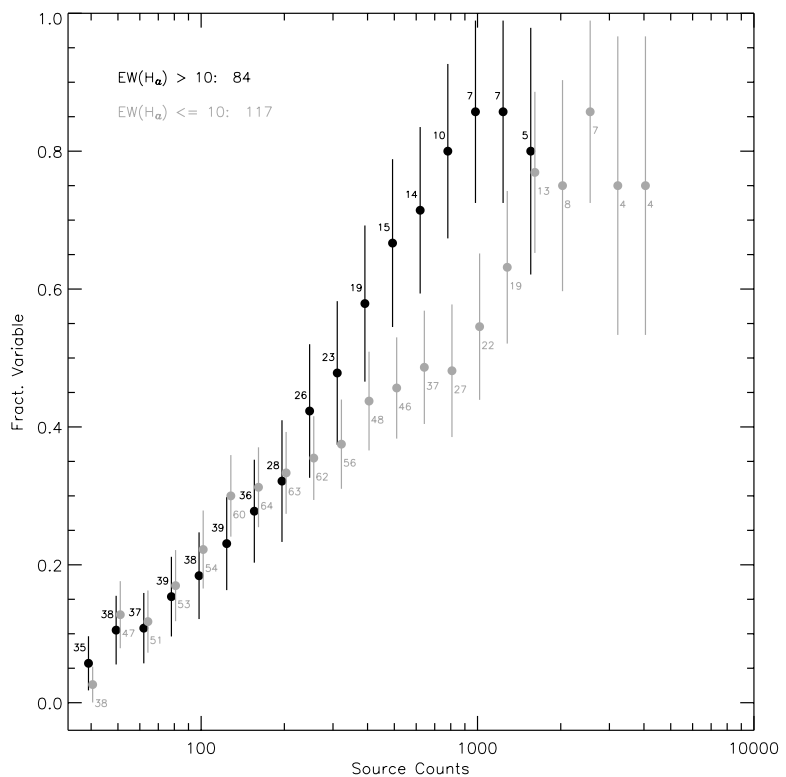

Fig. 6. Fraction of variable stars as a function of count statistics for stars with $\mathrm{H}_{\alpha}$ EW greater and smaller than 10 (i.e. CTTS and WTTS). Note that counts bin are centered at the plot symbol and are 0.8 dex wide, so that successive bins are not independent. Error bars are based on binomial statistics. Symbols referring to the two subsamples are slightly shifted in the horizontal direction with respect to each other so to avoid confusion. The total numbers of objects in the subsamples are given in the legend, while the numbers of objects from which each variability fraction is computed are shown beside the plotting symbols.

indicates the light-curve is almost certainly variable, while a $<1 \%$ value ( 87 sources) indicates probable variability, although up to $\sim 4(=420 \times 0.01)$ of the variable sources might actually be constant. If we are not interested in the individual sources but in the overall fraction of variable sources, we can place a lower limit on this quantity by computing the minimum number of variable sources as a function of probability threshold, $P_{\mathrm{th}}$ : $N_{\text {min }}=N\left(P_{\mathrm{KS}}<P_{\mathrm{th}}\right)-420 \times P_{\mathrm{th}}$. The maximum $N_{\min }$ is obtained for $P_{\text {th }}=15 \%$, for which $N\left(P_{\mathrm{KS}}<P_{\mathrm{th}}\right)=189$ and $N_{\min }=126$. We conclude that within our observation at least $30 \%(126 / 420)$ of the lightcurves are statistically inconsistent with constancy. This fraction is certainly a lower limit to the true number of variable sources, as our ability to tell a variable source from a constant one is influenced by photon statistics. This is clearly indicated by the dependence of the fraction of variable sources on source counts. If for example, we restrict the above analysis to sources with more than $100(500)$ counts, a total of $145(33)$ sources, we find that at least $54 \%(73 \%)$ of these are variable.

We next investigated whether different kinds of stars, classified from optical data, showed different variability fractions. From the previous discussion, it is clear that for a meaningful comparison, source statistics must be taken into account. Figure 6 shows the variability fraction, $f\left(P_{\mathrm{KS}}<1 \%\right)$, as a function of source counts for CTTS and WTTS, as distinguished by their $\mathrm{H}_{\alpha}$ equivalent width. Variability fractions are computed for sources with counts in intervals spanning 0.8 dex (because in the figure points are spaced by 0.1 dex, only one point every eighth is independent). Error bars on the variability fractions are estimated by assuming binomial statistics: $\delta f=\left[f \times(1-f) / N_{\text {src }}\right]^{1 / 2}$, where $N_{\text {src }}$ is the number of sources in each count bin. In addition to the expected increase in the variability fraction with source statistics, we note that CTTS appear to be more variable with respect to WTTS, at least when considering stars with 


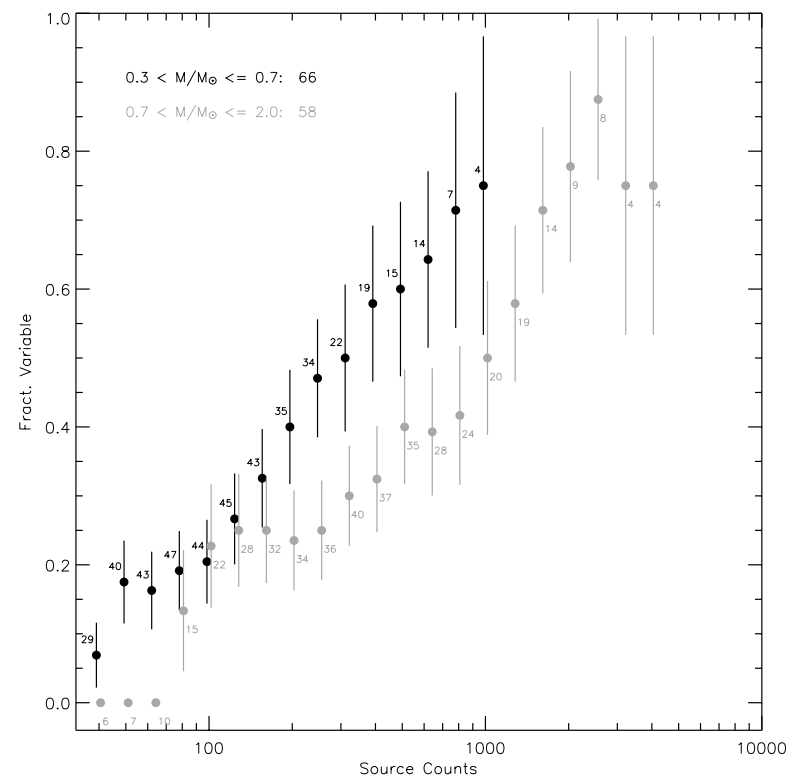

Fig. 7. Fraction of variable stars as a function of count statistics for stars in two different mass ranges: [0.3-0.7] $M_{\odot}$ and [0.7-2.0] $M_{\odot}$. Symbols and legend as in Fig. 6.

more than $\sim 200$ counts. Figure 7 shows a similar comparison for two mass segregated sub-samples: $0.3<M / M_{\odot}<0.7$ and $0.7<M / M_{\odot}<2.0$. Lower mass stars appear to be more variable, and again this difference is noticeable only for stars that are bright enough. We quantified the differences between variability fractions, by testing, for each count bin, the null hypothesis that the two samples are drawn from the same parent population. We chose the difference of the two observed variability fractions, $\Delta f$, as statistics. We then numerically computed the probability that a $\Delta f$ equal or larger than the observed one is obtained by randomly drawing pairs of numbers from binomial distributions appropriate to the two sample sizes but characterized by the same probability of observing a variable source, i.e. the variability fraction. Because this number is not well constrained, we conservatively took as our confidence level the minimum that is obtained varying this fraction between 0 and 1 . The results of these tests indicate that the differences in the variability fractions are statistically not highly significant: in the bin centered at $\sim 400$ counts, the low mass stars are more variable than higher mass stars with a $94 \%$ confidence, and CTTS are more variable than WTTS with a $92 \%$ confidenceand in the bin centered at $\sim 800$ counts. We thus consider these results as tentative. However we note that the significance of the difference between CTTS and WTTS in NGC 2264 is strengthened by the fact that a similar result, with a similar confidence, was also obtained by Flaccomio et al. (2000) using totally independent ROSAT data.

\subsection{Spectral analysis}

We have analyzed the X-ray spectra of the 199 sources with more than 50 detected photons. Spectral fits were performed with XSPEC 11.3 and with several shell and TCL scripts to automate the process. For each source we fit the data in the [0.5-7.0] keV energy interval ${ }^{10}$ with several model spectra: one and two isothermal components (APEC), subject to photoelectric

\footnotetext{
${ }^{10}$ Events with energies below $0.5 \mathrm{keV}$ were not used because of uncertainties in the calibration resulting from the time-dependent degradation of the ACIS quantum efficiency at low energies.
}

Table 5. Initial conditions for XSPEC models.

\begin{tabular}{lccc}
\hline Model name & $N_{\mathrm{H}}\left[\mathrm{cm}^{-2}\right]$ & $k T_{1} / k T_{2}$ & Abund. \\
\hline $1 \mathrm{~T}$ & $0.0,10^{22}$ & $0.5 /-, 1.0 /-, 2.0 /-, 10.0 /-$ & 0.3 \\
$2 \mathrm{~T}$ & $0.0,10^{22}$ & $0.4 / 1.0,1.0 / 3.0,0.5 / 2.0$ & 0.3 \\
$2 \mathrm{Tab}$ & $0.0,10^{22}$ & $0.4 / 1.0,1.0 / 3.0,0.5 / 2.0$ & 0.3 \\
$1 \mathrm{~T}_{A \mathrm{v}}$ & $1.6 \times 10^{21} A_{\mathrm{V}}$ & $0.5 /-, 1.0 /-, 2.0 /-, 10.0 /-$ & 0.3 \\
$2 \mathrm{~T}_{A \mathrm{v}}$ & $1.6 \times 10^{21} A_{\mathrm{V}}$ & $0.4 / 1.0,1.0 / 3.0,0.5 / 2.0$ & 0.3 \\
$2 \mathrm{Tab}_{A \mathrm{v}}$ & $1.6 \times 10^{21} A_{\mathrm{V}}$ & $0.4 / 1.0,1.0 / 3.0,0.5 / 2.0$ & 0.3 \\
\hline
\end{tabular}

Multiple values indicate that all combinations of initial values were adopted. Values in italic indicate that fit parameters were fixed to those values.

absorption from interstellar and circumstellar material (WABS). Plasma abundances for one-temperature (1T) models were fixed at 0.3 times the solar abundances (Wilms et al. 2000), while they were both fixed at that value and treated as a free parameter for two-temperature (2T) models. The absorbing column densities, $N_{\mathrm{H}}$, were both left as a free parameter and fixed at values corresponding to the optically/NIR determined extinctions, when available: $N_{\mathrm{H}}=1.6 \times 10^{21} A_{\mathrm{V}}$ (Vuong et al. 2003). A total of three or six models were thus fit for each source depending on the availability of optical extinction values. For each model, spectral fits were performed starting from several initial conditions for the fit parameters as indicated in Table 5. For example for isothermal models with free $N_{\mathrm{H}}$, two values of $N_{\mathrm{H}}$ and four values of $k T$ were adopted as initial conditions, for a total of eight distinct fits. For each model the adopted fit parameter set was chosen from the model fit that minimizes the $\chi^{2}$. This procedure was adopted in order to reduce the risk that the $\chi^{2}$ minimization algorithm used by XSPEC finds a relative minimum.

Next, we considered which of the available model fits for each source (three or six, reference model names are given in Table 5) was the most representative of the true source spectrum, and thus the one to be adopted for the following considerations. The goal was twofold: to characterize the emitting plasma, in order to investigate its origin, and to determine accurate intrinsic band-integrated X-ray luminosities. Crucial for this latter step is to determine extinction $\left(N_{\mathrm{H}}\right.$, see Sect. 4.3). Generally speaking, models must have enough components to yield statistically acceptable fits according to the $\chi^{2}$ or, equivalently, the null probabilities (n.p.) that the observed spectra are described by the models. However, models with too many free parameters with respect to the spectra statistics, while formally yielding good fits, will not be constrained by the data and will yield limited physical information. A particularly severe problem with CCD-quality (ACIS) low-statistic spectra is the degeneracy between absorption and temperature: an equally good fit can be often obtained with a cool plasma model with a large emission measure but suffering high absorption, or with a warmer temperature and a lower extinction. It is therefore desirable to check the $N_{\mathrm{H}}$ obtained from the fits with independent information from optical/NIR data. Figures 8 and 9 show the cumulative distribution of the n.p. for spectral fits performed with four different models, respectively for faint and bright sources $(50<\mathrm{cnts}<300$, cnts $>300)$. The models are 1T, 2T, and, for sources with independent $N_{\mathrm{H}}$ estimates, $1 \mathrm{~T}_{A \mathrm{v}}$ and $2 \mathrm{~T}_{A \mathrm{v}}$. In this kind of plot the distribution for a perfectly adequate spectral model should follow the diagonal, that for an oversimplified model should fall below the diagonal, and that for an over-specified model should lie above. For faint sources, 1T model appears perfectly adequate. Fixing the $N_{\mathrm{H}}$ to the optically determined value worsens the agreement somewhat but still results in fits that are, when 


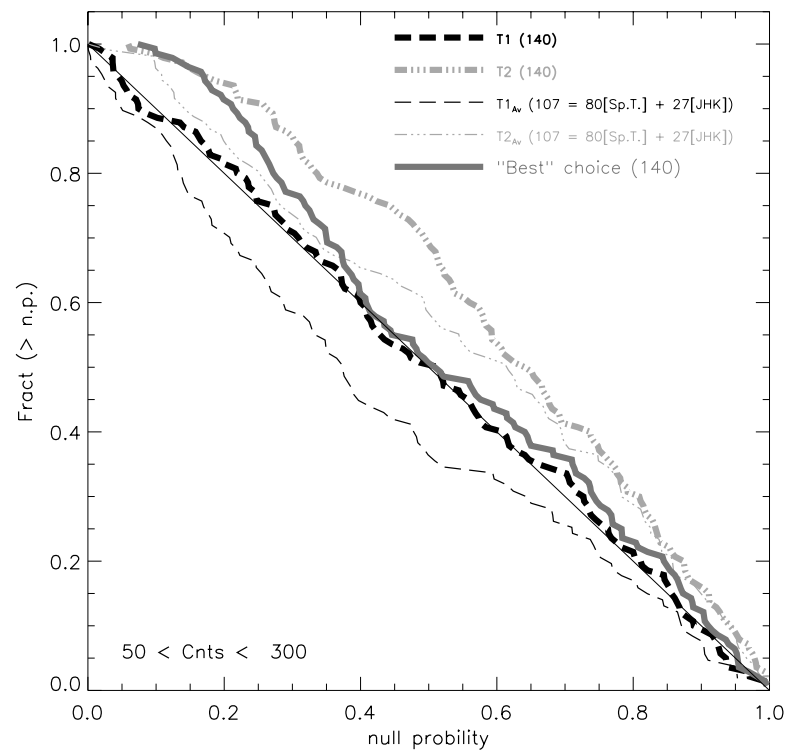

Fig. 8. Cumulative distribution of null probabilities resulting from $\mathrm{X}$-ray spectral fits. The curves refer here to X-ray sources with more that 50 counts (the minimum for which we performed spectral fits) and less than 300 counts. The different lines refer to different physical models (one and two temperature, with free of fixed $N_{\mathrm{H}}$ ) as indicated in the legend, along with the number of sources that enter in each distribution. For the models with fixed $N_{\mathrm{H}}$, we also indicate the number of $N_{\mathrm{H}}$ derived from optical spectral types + photometry and of those derived from 2MASS $J, H$, and $K$ photometry. The gray thick line refers to the "best" choice of models described in Sect. 4.2.

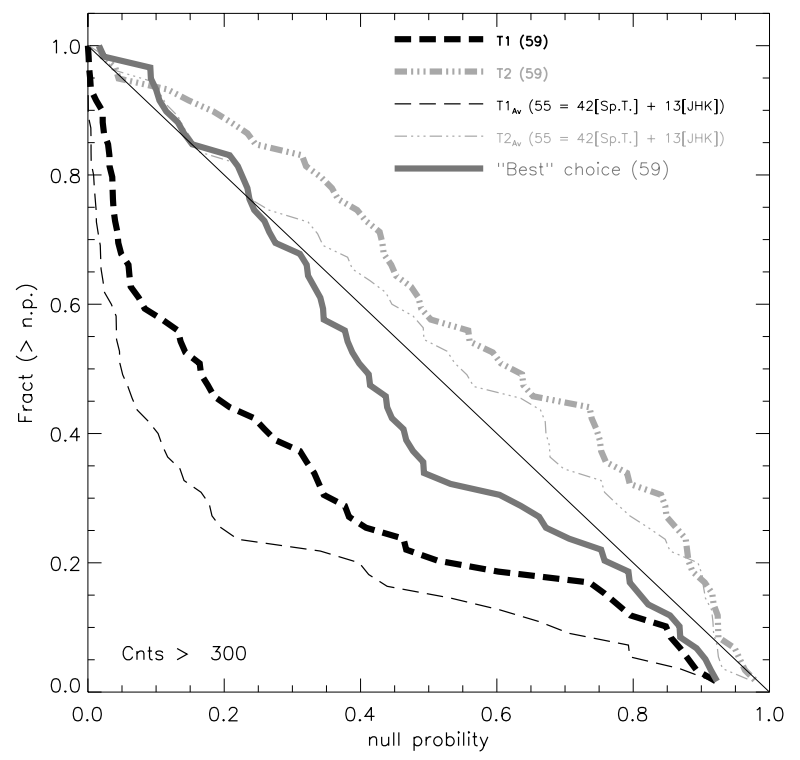

Fig. 9. Same as Fig. 8 for sources with more than 300 counts.

considered individually, acceptable for the most part. A $2 \mathrm{~T}$ model with free $N_{\mathrm{H}}$ appears to be too complicated (and therefore unconstrained), while a $2 \mathrm{~T}$ model with fixed $N_{\mathrm{H}}$ is more acceptable, but still on average too sophisticated for the quality of these low-statistic spectra. For brighter sources we notice instead that $1 \mathrm{~T}$ models are on average not favored, while $2 \mathrm{~T}$ models are still not always needed.

For most sources more than one spectral model is statistically acceptable. We choose a best-guess model as the simplest that still gives a statistically acceptable fit. The compelling reason to choose this approach is the mentioned degeneracy between temperature and absorption. Note for example that, from Fig. 8, one could be tempted to always choose 1T models with free $N_{\mathrm{H}}$ for faint sources, as these models appear to be statistically perfectly adequate for representing the spectra. However, when examined individually, many of these 1T spectral fits have rather degenerate fit solutions, i.e. large and correlated uncertainties on the $N_{\mathrm{H}}$ and $k T$ values, also implying large uncertainties on unabsorbed fluxes. This degeneracy can be broken by using the additional information on absorption coming from optical/NIR data, when available and compatible with the $\mathrm{X}$-ray spectra.

After some experimenting, we chose our best-guess model according to the following empirical scheme: if n.p. $\left(1 \mathrm{~T}_{A_{\mathrm{v}}}\right)>$ $20 \%$ we chose the $1 \mathrm{~T}_{A \mathrm{v}}$ model. Otherwise, if n.p.(1T) $>20 \%$ we chose the $1 \mathrm{~T}$ model (with free $N_{\mathrm{H}}$ ). If both of the previous tests for isothermal models failed, we tried with two temperature models, this time lowering our n.p. threshold to 5\%. First we tried the $2 \mathrm{~T}_{A \mathrm{v}}$ model (n.p. $\left(2 \mathrm{~T}_{A_{\mathrm{v}}}\right)>5 \%$ ) and then the $2 \mathrm{~T}$ model (n.p. $(2 \mathrm{~T})>5 \%$ ). As a last resort, in case none of the previous models could be adopted, we chose the $2 \mathrm{Tab}$ model (free $N_{\mathrm{H}}$, free abundances). Although the above scheme was designed so as to favor simple models, the different n.p. thresholds resulted in four cases (sources \#257, \#275, \#280, and \#300) in adopting $2 \mathrm{Tab}$ models that were either statistically worse or comparable to simpler $1 \mathrm{~T}, 1 \mathrm{~T}_{A \mathrm{v}}$, or $2 \mathrm{Tab}_{A \mathrm{v}}$ models. We therefore adopted these latter. After careful examination of individual fits, the spectral models adopted for four more sources (\#97, \#104,\#127, and \#241) were modified. In these cases the automatic choice would result in unphysical, unusual, or unconstrained temperatures and absorptions, whereas our adopted models are both statistically and physically acceptable ${ }^{11}$.

The solid gray lines in Figs. 8 and 9 refer to the final choice of best-guess models. Table 6 lists the end result of our spectral analysis: we report the adopted model, the source of the adopted $N_{\mathrm{H}}$, the null probability, the plasma temperature(s) and normalization(s), the observed and absorption corrected fluxes in the [0.5-7] keV band.

In summary, out of the 199 sources with more than 50 counts, we adopted isothermal models in 147 cases and two component models in the remaining 52 cases. For 138 sources we adopted a spectral fit in which the $N_{\mathrm{H}}$ was fixed, in 101 cases to the value estimated from the optically determined $A_{\mathrm{V}}$ and in 37 cases from NIR photometry. In the remaining 61 cases, $N_{\mathrm{H}}$ was treated as a free fit parameter. Out of these 61 cases, independent estimates of extinction were available from optical and NIR data in 21 and 3 cases respectively, but our algorithm (or, for sources \#97 and \#127, our choice) preferred the spectral fit with free $N_{\mathrm{H}}$. We examine these cases more in detail to assess the ambiguities in the model fits and the consequences on the derived X-ray fluxes.

Figure 10 illustrates one such case: source \#375. Here the extinction determined from the $1 \mathrm{~T}(k T=1.3 \mathrm{keV})$ spectral fit (90\% confidence interval: $N_{\mathrm{H}}<2 \times 10^{20} \mathrm{~cm}^{-2}$ ) is lower than that estimated from the $A_{\mathrm{V}}\left(9 \times 10^{20} \mathrm{~cm}^{-2}\right)$. Fixing the $N_{\mathrm{H}}$ to that

11 For source \#241 we changed the $2 \mathrm{~T}$ model (n.p. $=32 \%, k T_{1}=$ $0.057_{0.022}^{7.8} \mathrm{keV}, k T_{2}=0.57_{0.30}^{1.30} \mathrm{keV}, N_{\mathrm{H}}=13_{1.8}^{21} \times 10^{21} \mathrm{~cm}^{-2}$ ) to a $1 \mathrm{~T}$ model (n.p. $=17 \%, k T=0.60_{0.38}^{0.84} \mathrm{keV}, N_{\mathrm{H}}=3.3_{0.0}^{6.5} \times 10^{21} \mathrm{~cm}^{-2}$ ). For source \#104 we changed the $1 \mathrm{~T}$ model (n.p. $=40 \%, k T=0.43_{0.29}^{0.73} \mathrm{keV}$, $N_{\mathrm{H}}=3.0_{0.78}^{5.6} \times 10^{21} \mathrm{~cm}^{-2}$ ) to a $2 \mathrm{~T}_{A \mathrm{v}}$ model (n.p. $=32 \%, N_{\mathrm{H}}\left(A_{\mathrm{V}}\right)=$ $0.0 \mathrm{~cm}^{-2}$ and reasonable temperatures). For sources \#97 and \#127 we changed from $1 \mathrm{~T}_{A v}$ models $(p=68 \%$ and $p=21 \%, k T=54 \mathrm{keV}$ and $k T=19 \mathrm{keV}$, both with unconstrained uncertainties) to 1T models $(p=97 \%$ and $p=56 \%, k T=4.7 \mathrm{keV}$ and $k T=3.5 \mathrm{keV})$. 


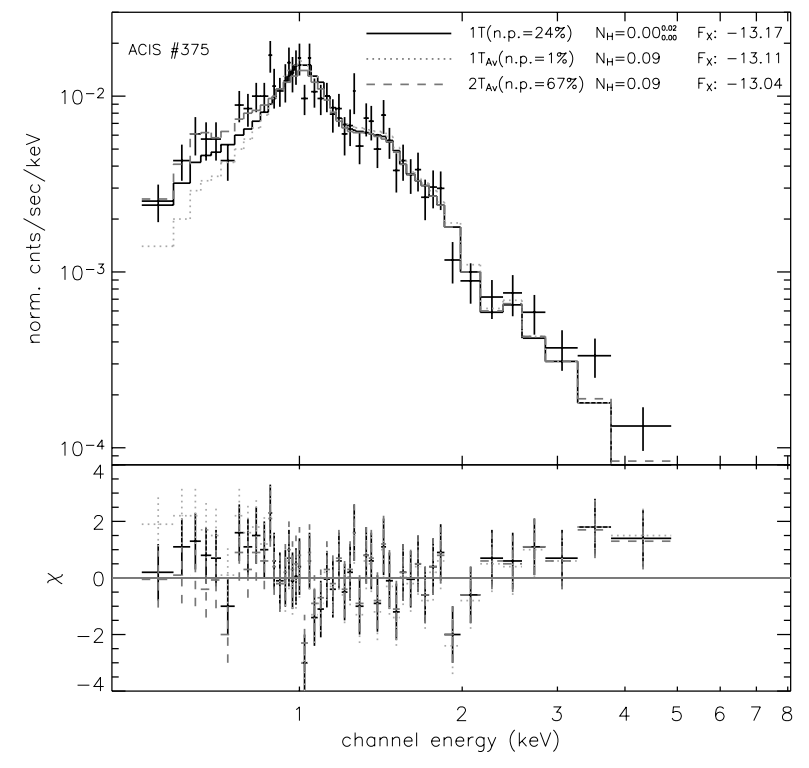

Fig. 10. Upper panel: spectrum of source \#375 with overlaid three different spectral models, $1 \mathrm{~T}$ with both free and fixed $N_{\mathrm{H}}$ (solid and dotted lines, respectively) and $2 \mathrm{~T}$ with fixed $N_{\mathrm{H}}$ (dashed line). Null probabilities, $N_{\mathrm{H}}$ values (in units of $10^{22} \mathrm{~cm}^{-1}$ ), and unabsorbed fluxes for each model are given in the legend. Note that the $1 \mathrm{~T}$ and $2 T_{A \mathrm{v}}$ models are both statistically acceptable. Lower panel: residuals for the three models in terms of sigmas (error bars all of unit size).

value yields an unsatisfactory isothermal fit. However, adding a second cool isothermal component to the spectral model $(k T=$ $0.25 \mathrm{kev}$ ), a good fit can be obtained even when fixing the $N_{\mathrm{H}}$. This is quite a typical example of the degeneracy in the fitting of sources with low/moderate statistics. We can estimate the uncertainty in the X-ray unabsorbed fluxes derived from the spectral fits due to this degeneracy, as the difference between the fluxes derived from the two acceptable fits: for source \#375, for example, this is 0.14 dex. More generally, acceptable fits with $N_{\mathrm{H}}$ fixed to the optical/NIR values could be obtained in 22 of the 24 cases in which our procedure preferred the spectral fit with free $N_{\mathrm{H}}$. In five cases ${ }^{12}$, among which the one described above, in order to reconcile the observed spectra with the optical extinction, an additional cool thermal component $(k T=0.22-0.34)$ would be required to compensate for the higher $N_{\mathrm{H}}$. If for these five sources the true $N_{\mathrm{H}}$ were the optically derived ones, we are underestimating the unabsorbed fluxes by $0.14-0.37$ dex (mean 0.2 dex) by choosing the $1 \mathrm{~T}$ fit with free (lower) $N_{\mathrm{H}}$. For the other 17 sources, fixing the $N_{\mathrm{H}}$ would not require an additional component: reasonable fits (n.p. $>5 \%$ ) were obtained even with the same models (1T in 16 cases, $2 \mathrm{Tab}$ for source \#187). In 10 of these 17 cases the adopted fits with free $N_{\mathrm{H}}$ lead to smaller absorptions and slightly hotter $(0.2-0.3 \mathrm{keV})$ temperatures (the cool temperature for \#187), while the opposite happens in the 7 other cases. Had we fixed the $N_{\mathrm{H}}$ to the optical values in these 17 cases, we would have obtained unabsorbed fluxes on average $\sim 0.03$ dex larger and always within 0.20 dex (in either direction) of the adopted ones.

From this discussion of the 22 cases with most ambiguous extinctions ( $14 \%$ of the sources for which we have optical/NIR $N_{\mathrm{H}}$ ), we conclude that (i) typical uncertainties in the unabsorbed flux are less than 0.2 dex and that (ii) individual $\mathrm{X}$-ray fluxes corrected using an $N_{\mathrm{H}}$ from spectral fits can be as wrong as

12 Sources \#190, \#340, \#352, \#375, \#391.

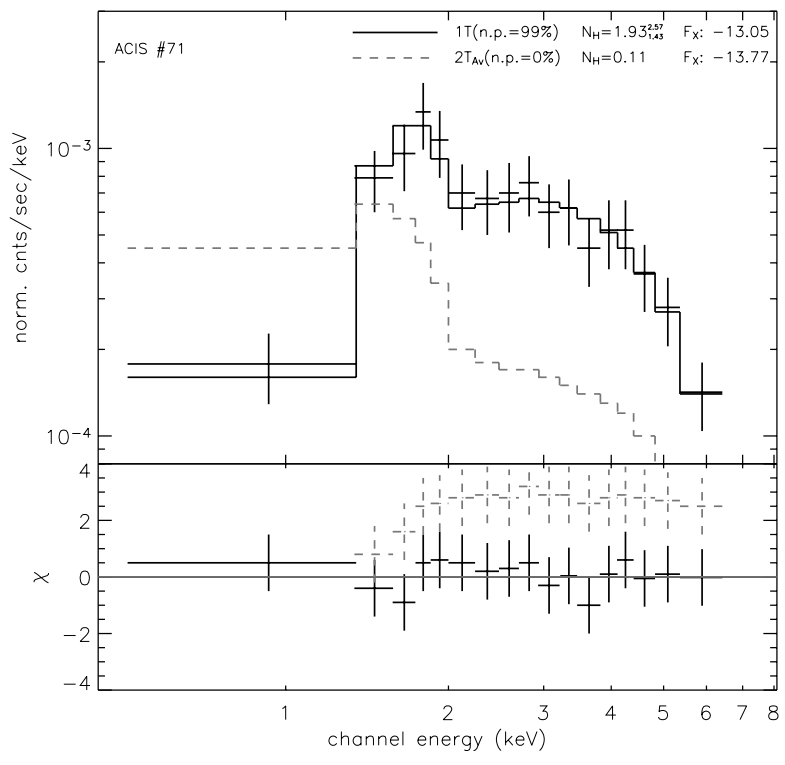

Fig. 11. Some as Fig. 10 for source \#71. In this case we plot the $1 \mathrm{~T}$ model with free $N_{\mathrm{H}}$ and the $2 \mathrm{~T}$ model with $N_{\mathrm{H}}$ fixed to the optical value. Note how even adding a second component, it is impossible to reconcile the observed spectrum with the $N_{\mathrm{H}}$ implied by the optical absorption and a standard absorption law.

$\sim 0.4$ dex, if the cool temperature is altogether missed by the spectral fit with a consequent underestimation of the $N_{\mathrm{H}}$.

Two more sources have incompatible X-ray and optical extinctions according to our choice of best model. Source \#234 did not enter into the previous discussion because it has the least acceptable fits of the whole sample, at most n.p. $=1.6 \%$ for a $2 \mathrm{~T}$ model with free $N_{\mathrm{H}}$ (note, however, that with an adequate spectral model, we would expect three sources out of 199 to have lower n.p.). Adopting the optical $N_{\mathrm{H}}\left(1.8 \times 10^{21}\right)$ would result in an unabsorbed flux that is only $0.07 \mathrm{dex}$ larger with respect to the one obtained leaving $N_{\mathrm{H}}$ as a free fit parameter $\left(N_{\mathrm{H}}=\right.$ $\left.0.59_{0.16}^{0.78} \times 10^{21}\right)$. Source \#71 is a more interesting case. Figure 11 shows its spectrum, with spectral fits obtained with free and fixed $N_{\mathrm{H}}$. The X-ray-derived extinction $\left(N_{\mathrm{H}}=19_{14}^{26} \times 10^{21}\right)$ is about 17 times larger than estimated from the optical reddening $\left(N_{\mathrm{H}}=1.1 \times 10^{21}\right)^{13}$. The discrepancy is highly significant and independent of the considered spectral model. We note that the detected emission from this source is dominated by a powerful flare, with a peak count rate $\sim 100$ times what it was before and after the flare. A possible scenario to explain the high absorption might involve a solar-like coronal mass ejection associated with the flare, providing the additional absorbing material. This hypothesis has been formulated by Favata \& Schmitt (1999) to explain a more modest increase in $N_{\mathrm{H}}$ during a powerful flare observed on Algol. Further discussion of the physical interpretation of spectral fit results is deferred to Sect. 6 .

\footnotetext{
13 For this source we adopted the spectral type (K2) from Dahm \& Simon (2005) and the photometry $\left(R_{\mathrm{c}}-I_{\mathrm{c}}=0.62\right)$ from Lamm et al. (2004). Independent photometry and spectral types are available from Rebull et al. (2002), Lamm et al. (2004), and Dahm \& Simon (2005). The $R_{\mathrm{c}}-I_{\mathrm{c}}$ color ranges between 0.55 and 0.62 and spectral types between G9 and K2. The possible range in $N_{\mathrm{H}}=1.6 \times 10^{21} A_{\mathrm{V}}$ is thus $0.57-1.64 \times 10^{21}$, i.e. between $\sim 9$ and $\sim 46$ times the value derived from the X-ray spectrum, considering its $90 \%$ confidence interval.
} 


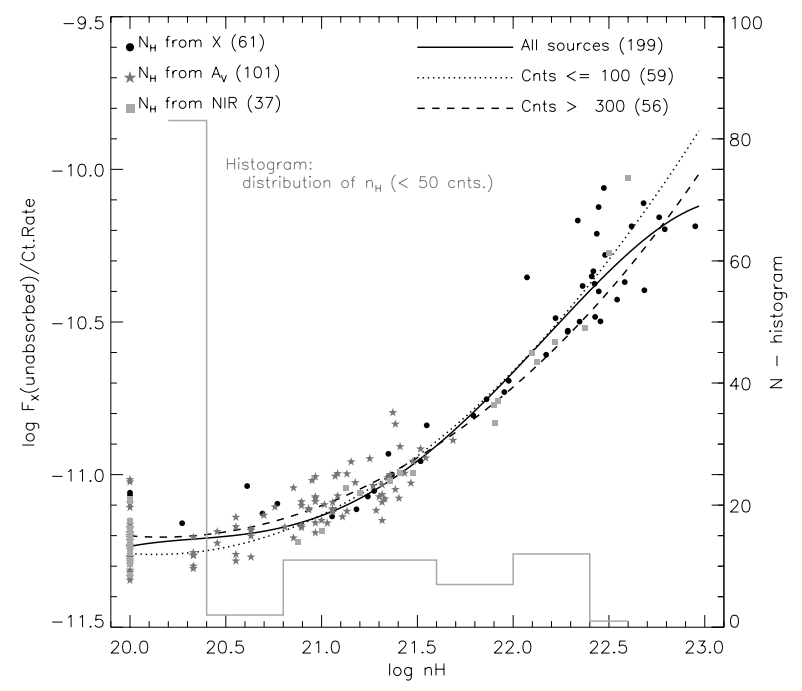

Fig. 12. Count-rate to unabsorbed flux conversion factor as a function of $N_{\mathrm{H}}$ as derived from spectral fits. Circles refer to the spectral fits in which $N_{\mathrm{H}}$ was treated as a free parameter, star-symbols and squares to fits in which $N_{\mathrm{H}}$ was fixed to the values derived from the optical $A_{\mathrm{V}}$ or from the NIR photometry, respectively. The three curves indicate polynomial fits to the data obtained for the whole sample of 199 sources and for subsamples of sources with less than 100 counts (59 sources) or more than 300 counts ( 56 sources). The gray histogram (vertical scale on the right hand side) shows the distribution of absorption values, derived from the $A_{\mathrm{V}}$ or the NIR CCD, of X-ray sources with less than 50 counts, for which count-rate to flux conversion factor was determined using the polynomial fit to faint sources ( $<100$ counts, dotted curve).

\subsection{X-ray luminosities}

Extinction-corrected X-ray luminosities in the [0.5-7.0] keV band were estimated for all sources for which an indication of extinction was available. For the 199 sources with more than 50 counts and for which spectral fits were performed, we computed $L_{\mathrm{X}}$ from the $F_{\mathrm{X}}[u]$ (unabsorbed fluxes) column in Table 6, adopting a distance of $760 \mathrm{pc}$, adequate for NGC 2264 members. For the fainter sources, for which spectral fits were not performed, we derived a count-rate to unabsorbed flux conversion factors using the results of the spectral fits for the brighter stars. Figure 12 shows the run of the flux/rate ratio vs. $N_{\mathrm{H}}$ for this sample. Different symbols indicate different origins of the absorption values (see previous section). We then performed polynomial fits to the data points for the whole sample, for the 56 sources with more than 300 counts, and for the 59 sources with less 100 counts. Results are shown in Fig. 12. Because the sources for which we want to determine fluxes have $<50$ counts, we adopt the latter fit as our relation between $N_{\mathrm{H}}$ and the conversion factor:

$\log \frac{F_{\mathrm{X}}(u)}{\text { Ct.Rate }}=58.62-6.942 \times \log N_{\mathrm{H}}+0.1724 \times\left(\log N_{\mathrm{H}}\right)^{2}$

where the units are $\operatorname{ergs} \mathrm{cm}^{-2}$. The $1 \sigma$ dispersion of the datapoints around this relation is $0.1 \mathrm{dex}$, which can be taken as an upper limit to the error on the source flux introduced by ignoring the spectral shape of faint sources. From the observed count-rates, we thus estimated fluxes for $127 \mathrm{X}$-ray sources with $<50$ counts and with $N_{\mathrm{H}}$ estimates coming from either the $A_{\mathrm{V}}$ or the $J H K$ photometry. The gray histogram in figure 12 shows the distribution of $N_{\mathrm{H}}$ for these sources. Results are reported in Table 1. Note that we do not quote X-ray fluxes for faint sources without an independent extinction estimate. Upper limit fluxes for 425 X-ray undetected stars in the ACIS FOV and with an absorption estimate (out of a total of 1463) were similarly computed from the upper-limits to the count rates.

\section{Results - membership}

In this section we define a sample of 491 likely NGC 2264 members within the FOV of our ACIS observation. The X-ray sources account for 408 objects while the remaining 83 are selected on the basis of the strength of $\mathrm{H}_{\alpha}$ line and of optical variability as described in Sect. 3.2. The likely members are indicated in Table 3 by an asterisk following the identification number. We first consider the possibility of extragalactic contamination and then discuss the nature of X-ray sources with and without optical/NIR counterparts separately.

\subsection{Extragalactic $X$-ray sources}

Extragalactic contamination is expected only among X-ray sources that lack an optical and/or NIR counterpart. We reach this conclusion by considering the 489 ACIS sources of a probably non stellar origin detected in the Chandra Deep Field North (CDFN; Alexander et al. 2003; Barger et al. 2003) ${ }^{14}$. After defining random positions in our FOV for each CDFN source, we compared their observed count rates with upper limits computed from our ACIS data at those positions (see Sect. 3.1). We then selected CDFN sources that would have been detected with our exposure: a total of $139 \pm 3$ objects ${ }^{15}$. Next, we compared the positions of these simulated CDFN AGNs in the optical ( $I$ vs. $R-I$ ) and NIR ( $H$ vs. $H-K$ ) color-magnitude diagrams ${ }^{16}$ with those of the X-ray sources in the NGC 2264 exposure that could be placed in the same diagrams. These plots show that AGNs are considerably fainter on average than our identified X-ray sources that can be placed in either of these diagrams, and only two or three of the AGNs that we could have detected occupy positions that overlap with the loci where our X-ray sources are found. Moreover, in this comparison we have totally neglected the effect of extinction. Because of the dark cloud in our line of sight, the number of AGNs we are sensitive to is certainly much reduced with respect to the above estimate, and their optical and NIR luminosities should also be considerably reduced. We therefore conclude that the contamination of AGNs to the sample of $\mathrm{X}$-ray-selected probable members with optical/NIR identification is negligible.

\subsection{X-ray sources with optical/NIR counterparts}

In Fig. 3 we show the optical CMD for the 300 X-ray sources that can be placed in such a diagram and for the 83 other undetected problable members discussed in Sect. 3.2. Note that the X-ray sources are found for the most part in the locus expected for NGC 2264 members (i.e. the previously defined cluster locus). However some X-ray sources lie below that locus, their position being compatible with the one expected for MS foreground stars. Very few, if any, of the X-ray sources in this diagram may be background MS stars, which are expected to lie below the 1 Gyr isochrone. In order to reduce contamination in the sample of likely members to a minimum, we excluded X-ray sources that lie outside the cluster locus, i.e. below the $10^{7.1} \mathrm{Myr}$

\footnotetext{
14 We excluded 14 sources identified as stars by Barger et al. (2003).

15 Mean value and $1 \sigma$ uncertainty result from repeating the experiment 10 times, each time varying the position of simulated extragalactic sources.

16 The NIR photometry of the CDFN sources was taken from 2Mass.
} 
isochrone and to the right of the $0.8 M_{\odot}$ evolutionary track, but we made an exception for two sources: (i) \#305 ( $I=17.28$, $R-I=0.98)$, associated with the IRAS source IRS-1, classified as a Class I object by Margulis et al. (1989) but more recently suggested as a deeply embedded B star in a more evolved evolutionary stage (Schreyer et al. 2003) ${ }^{17}$; (ii) \#309 $(I=16.45$, $R-I=0.76$ ), a known member, because indeed the peculiar and well-studied accreting binary system KH 15D (e.g. Hamilton et al. 2005; Herbst \& Moran 2005; Dahm \& Simon 2005). The 8 sources that lie to the right of the $0.1 M_{\odot}$ track are considered likely members, either of very low (sub)stellar mass or else very absorbed. Since we lack $A_{\mathrm{V}}$ values from optical spectra, we can check these two possibilities using our X-ray data and the JHK photometry. Out of these 8 sources, 4 (\#125, \#192, \#251, \#273) are highly absorbed as indicated by their X-ray spectra (or hardness ratios) and/or their positions in the $J-H$ vs. $H-K$ diagram. Another three sources (\#215, \#316, \#358) however appear to have negligible absorption and are good candidates for detected brown dwarfs.

Only 10 X-ray sources (out of 300) are excluded as members because they are incompatible with our cluster locus in the optical CMD and might be associated with field stars ${ }^{18}$. Among them only one (source \#258, an X-ray faint, $L_{\mathrm{X}} / L_{\mathrm{bol}}=-4.8$, and soft G5 star) could be placed in the HR diagram of Fig. 5, where it lies on the 100Myr isochrone. Although we exclude these 10 sources from our sample of likely members, some of them might actually be members.

- In Fig. 3 six very likely members, 5 undetected, and one detected CTTS, are found below the cluster locus, indicating that some members do have a position in the optical CMD that differs from expectations.

- The spatial distribution of these 10 sources (Fig. 13) is similar to that of cluster members and not uniform, as expected from foreground stars.

- The ACIS spectra and hardness ratios indicate that 5 of these sources suffer high extinction in the X-ray band, which is incompatible with foreground MS star (but not with background giants).

Our next step was to statistically estimate the number of foreground stars that, if present in our FOV and detected by ACIS, contaminate our list of $290(=300-10)$ X-ray detected clusterlocus candidate members, as well as the $10 \mathrm{X}$-ray sources outside of the cluster locus. We considered the volume-limited stellar samples of the NEXXUS survey (Schmitt \& Liefke 2004), extracting from it a nearly complete sample of stars of spectral types $\mathrm{F}$ to $\mathrm{M}$ within $6 \mathrm{pc}$ from the Sun. These stars are well characterized optically and have known distances and X-ray luminosities as measured with ROSAT. We estimated the number of foreground stars, $N_{\mathrm{fg}}$, in our FOV assuming that the spatial density of field stars in the direction of NGC 2264 is uniform and equal to that in the solar neighborhood ${ }^{19}$. We next randomly drew $N_{\text {fg }}$ stars from the NEXXUS sample and assigned them random distances, $d$, in the range [0-760] pc, according to a uniform stellar density. With these distances and assuming an isothermal X-ray spectrum with $k T=0.3 \mathrm{keV}$, we converted the NEXXUS X-ray luminosities to ACIS count rates using an

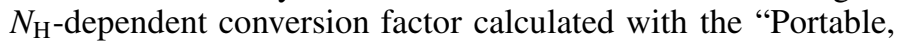

\footnotetext{
17 The ACIS spectrum indicates $N_{\mathrm{H}}=4.8 .8 .9 \times 10^{22}(90 \%$ confidence interval), corresponding to $A_{\mathrm{V}}=20-56$.

18 \#7, \#51, \#110, \#128, \#130,\#171, \#224, \#248, \#258, and \#267.

19 This is justified by the low equatorial longitude of NGC 2264: $2.2 \mathrm{deg}$.
}

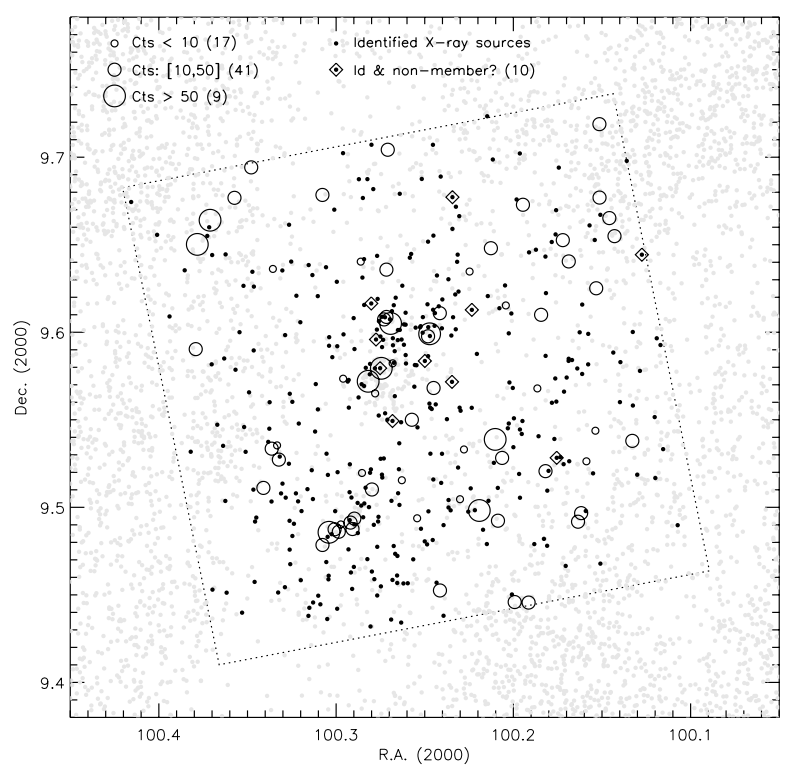

Fig. 13. Spatial distributions of sources around the ACIS FOV (the area within the dotted square). Light gray points: cataloged optical/NIR objects not detected in X-rays. Black symbols: X-ray detected objects. Squares with dots: X-ray sources that might be non-members because of their position in the optical CMD (Sect. 5). Empty circles with size indicating source statistics (see legend): unidentified X-ray sources.

Interactive Multi-Mission Simulator" (PIMMS). The hydrogen column density toward each star was estimated as $\rho \times d$, where the volume density $\rho$ was set to $0.3 \mathrm{~cm}^{-3}$ and the distance is expressed in $\mathrm{cm}^{20}$. We finally compared these simulated countrates with upper limits estimated at random positions in the ACIS FOV estimated using PWDetect (Sect. 3.1). This simulation was repeated 100 times, varying the sample of NEXXUS stars and their position in space. On average, the sample of foreground stars that we would have detected counts 20 stars, 15 of which should fall in the cluster locus and 5 outside of it. About half of the 10 stars outside the cluster locus are thus expected to be cluster members, thus reinforcing the arguments given in the previous paragraph. Within the cluster locus, only 5\% (15/290) of the X-ray sources are estimated to be non-members ${ }^{21}$. Given that eighty of the $\mathrm{X}$-ray sources in the cluster locus had not been previously selected as likely members because of either their $\mathrm{H}_{\alpha}$ emission or optical variability, we estimate that our sample contains $\sim 65$ new optically visible members.

Fifty-one more X-ray sources were identified with optical or NIR objects but could not be placed in the optical CMD (14 have $I$ magnitudes but no $R, 37$ are only detected in 2MASS). Extending the previous result, and because we can exclude an extragalactic nature for these sources, we consider these stars as additional candidate members. Only four of them had previous indication of membership from their $\mathrm{H}_{\alpha}$ or optical variability.

\subsection{Sources with no optical/NIR counterpart}

Sixty-seven ACIS sources are not identified with any object listed in the full-field optical/NIR catalogs we have

\footnotetext{
${ }^{20}$ With the chosen value of $\rho$, taking $N_{\mathrm{H}} / A_{\mathrm{V}}=1.6 \times 10^{21}$ and $d=$ $760 \mathrm{pc}$ (the assumed distance of NGC 2264), we obtain $A_{\mathrm{V}}=0.44$, in agreement with the median of values measured for NGC 2264 members. ${ }_{21}$ The fraction of non-members is expected to be higher among X-ray sources with $M / M_{\odot}>1$ and ages $\sim 10^{7} \mathrm{yr}$.
} 

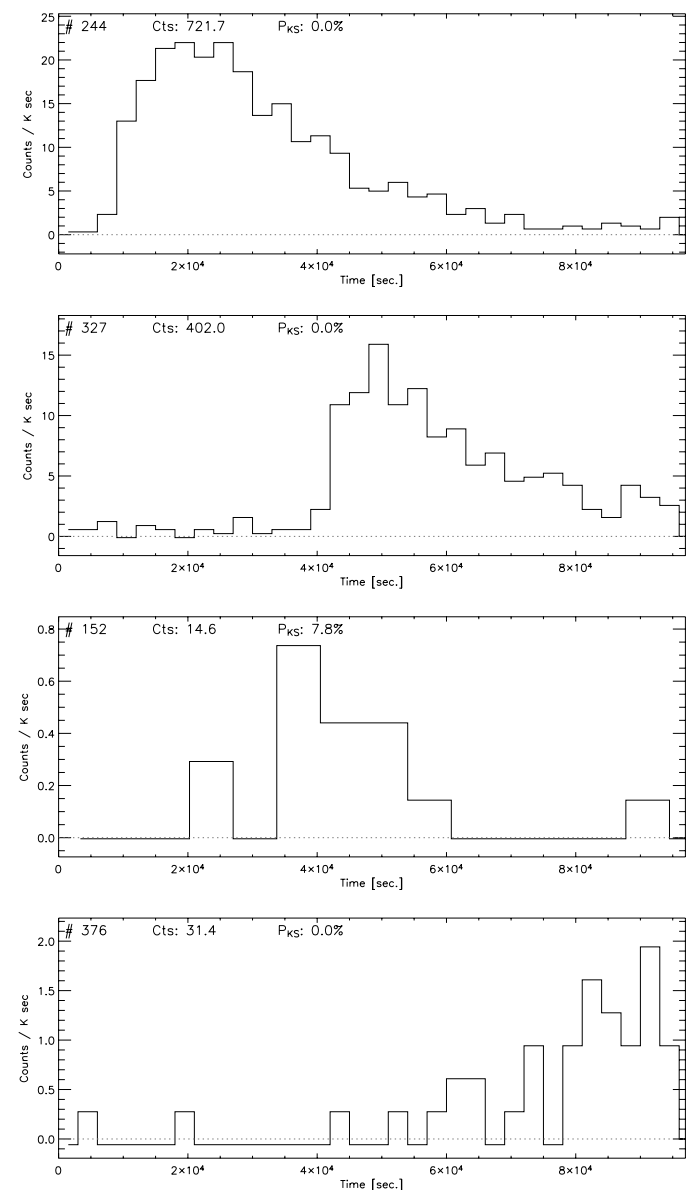

Fig. 14. Lightcurves for four "flaring" sources (\#152, \#244, \#327, and \#376) with no optical/IR counterpart. The background subtracted count rate is plotted vs. the time since the beginning of the observation. Source number, net counts, and the result of the KS variability test are given in the upper part of each panel.

considered $^{22}$. None of them was detected by Flaccomio et al. (2000) with the ROSAT HRI ${ }^{23}$. They have significantly fewer detected counts than identified sources. Fifteen of them have detection significance $<5.0 \sigma$, and the 10 expected spurious detections (cf. Sect. 2.2) are most likely found in this group. Nine have more than 50 counts and were subject to spectral analysis.

Depending on the optical depth of the background cloud, a number of AGNs are expected to be detected in our FOV and will be found among the non-identified sources (Sect. 5.1). It is therefore reasonable to ask whether the characteristics of our sources without counterparts are compatible with an AGN nature. The X-ray spectra of AGNs should be rather hard and well fit by power-law models with indexes between $\sim 0.9$ and $\sim 1.9^{24}$. Their lightcurves should be constant or slowly varying. They should be distributed uniformly in space or anti-correlated with the cloud

${ }^{22}$ Four of these X-ray sources were actually identified with MIR and/or mm sources in Sect. 3.3. Because these catalogs are not spatially complete, we here treat them as unidentified for uniformity.

23 In Table 3 our ACIS source \# 376 is associated with HRI source 138 due to the large uncertainty of the HRI positions; the HRI source is, however, closer to the brighter ACIS source \#375, with which it is most likely associated.

${ }^{24} 80 \%$ interval of the power indexes reported by Alexander et al. (2003) for the sources in the CDFN that we would have detected in our ACIS data and for which the $1 \sigma$ uncertainty in the index is lower than 0.1 .

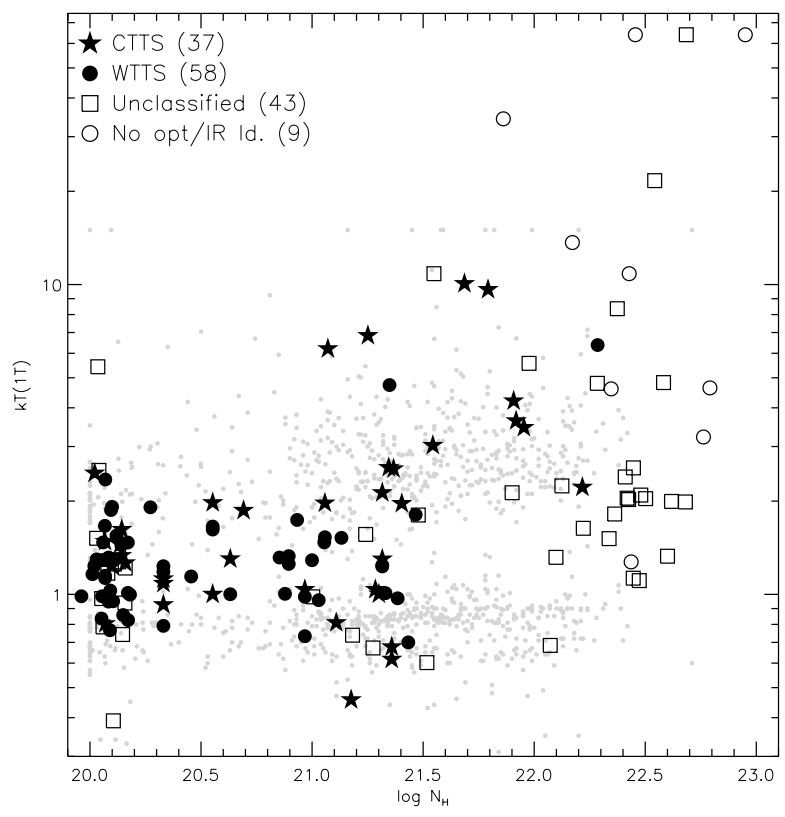

Fig. 15. Large black symbols: $k T$ vs. $N_{\mathrm{H}}$ for NGC 2264 sources for which isothermal models were adopted. We distinguish (see legend) CTTS and WTTS, stars that are identified with optical and/or NIR sources but for which the PMS class is unknown, and X-ray sources that are not identified with any known optical/NIR object. Note that stars with $\log N_{\mathrm{H}}<20.1$ have been shifted to that value and their horizontal position slightly randomized so that the symbols do not overlap completely. The small gray circles show for comparison the two temperatures derived by Getman et al. (2005) for a sample of $566 \mathrm{ONC}$ members observed by the COUP collaboration.

optical depth. With respect to this latter point, Fig. 13 shows, with different symbols, the spatial distribution of several classes of objects: X-ray sources without counterparts in three source count ranges, cross-identified X-ray sources, all the other X-ray undetected objects in our master catalog. This last group contains for the most part background field stars whose density is a good indicator of the optical depth of the molecular cloud. Note how the X-ray sources with counterparts, i.e. likely members, lie preferentially in front of or close to the cloud and have a highly structured distribution (cf., Lamm et al. 2004), with at least two concentrations in the south and toward the field center, corresponding to two well-known embedded sub-clusters roughly centered on IRS 1 (Allen's source, e.g. Schreyer et al. 2003) and IRS 2 (e.g. Williams \& Garland 2002).

We first discuss the nine unidentified sources with more than 50 counts: two of them (\#244, \#327), located in the IRS 1 and IRS 2 regions, show distinct long-lasting flares (Fig. 14) and are therefore most likely PMS stars or YSOs. As discussed in Sect. 3.3, source \#244 is actually identified with a SPITZER source and, although missing in the 2MASS catalog, it is rather bright in $\mathrm{K}$. The spectra of the other seven are compatible with both isothermal models and power-law models. Assuming isothermal models, they have higher extinctions and temperatures with respect to the 190 sources with counterparts and $>50$ counts (cf. Fig. 15): the median $N_{\mathrm{H}}$ is $2.7 \times 10^{22} \mathrm{~cm}^{-2}$, vs. $7 \times 10^{20} \mathrm{~cm}^{-2}$ and the median $k T$ is $13.7 \mathrm{keV}$ vs. $1.33 \mathrm{keV}$. Neither the $N_{\mathrm{H}}$ nor the $k T$ are incompatible with those of other highly extinct X-ray sources with counterparts (cf. Fig. 15), which we have argued are most likely not AGNs. Due to the high extinction, the $L_{X}$ of these sources (if at the distance of NGC 2264) is also high: median $\log L_{X}=30.4$ vs. $\log L_{X}=29.8$ 


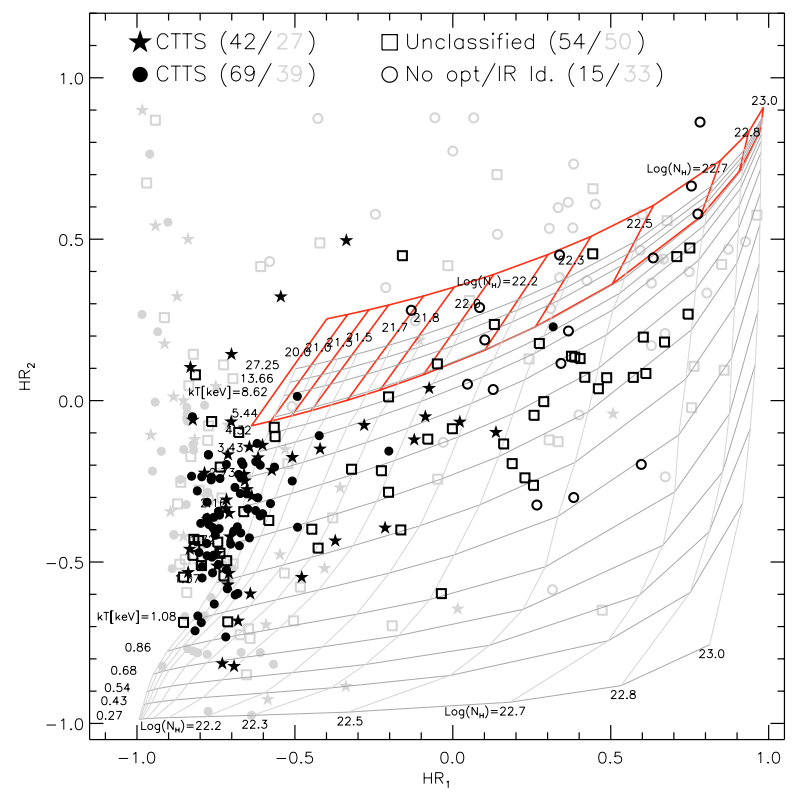

Fig. 16. $H R_{1}=(M-S) /(M+S)$ vs. $H R_{2}=(H-M) /(H+M)$, where $H$, $M$, and $S$ are the photon fluxes in the [500:1700] eV, [1700:2800] eV, and [2800:7000] eV bands. Sources for which $1 \sigma$ uncertainties on $H R_{1}$ and $H R_{2}$ are less than 0.3 are plotted in black and those with larger uncertainties in gray. Symbols are as in Fig. 15. The number of plotted sources in each class are given in the legend. The light gray grid indicates the expected loci of isothermal sources of a given $k T$ absorbed by interstellar matter of varying $N_{\mathrm{H}}$. Values of $k T \mathrm{~s}$ and $\log N_{\mathrm{H}}$ are given on the left and top/bottom of the grid. The upper thick-line grid indicates the region expected for AGNs (power-law spectra with indexes between 0.9 and 1.9).

for identified sources. If instead we assume that the correct models are power laws, the best-fit indexes range between $0.25_{-0.23}^{2.0}$ and $4.1_{2.5}^{6.5}$ (median 1.6). The two flaring sources have power indexes with $90 \%$ confidence above the range expected for AGNs, and the same is true also for source \# $162\left(\Gamma=4.1_{2.5}^{6.5}\right)$, which also lies in the IRS 2 region and is therefore likely to be a star (the isothermal fit yield a rather common $k T=1.27 \mathrm{keV}$ ). Two more (\# 228, \# 274) of the remaining six objects, although with rather hard and absorbed spectra, are clustered around IRS 2 and are therefore also likely to be YSOs. We thus conclude that $50 \%$ (5 out of 9) or more of the unidentified sources with more than 50 counts are likely to be stars.

Turning to the remaining 58 sources with less than 50 counts, two show flares (\#152 and \#376, Fig. 14) and are likely to be stars. The others, although maybe a little less spatially concentrated than sources with counterparts, seem to follow a similar distribution in the sky. We conclude that, rather qualitatively, a sizable fraction (on the order of $50 \%$ ) of them are associated with NGC 2264. This conclusion is corroborated by the distribution of unidentified sources in the $H R_{1}$ vs. $H R_{2}$ hardness ratio diagram, Fig. 16. We show for reference a grid of expected loci for absorbed isothermal spectra and the region where powerlaw sources with indexes between 0.9 and 1.9 should lie. Both grids are computed using PIMMS. We note that both $H R_{1}$ (most sensitive to $N_{\mathrm{H}}$ ) and $H R_{2}$ (most sensitive to $k T$ ) are on average significantly different for sources with and without counterparts, indicating that non-identified sources are on average characterized by hotter and more absorbed emission. However, the region occupied by about half of the unidentified sources is also occupied by a number of absorbed sources with counterparts, i.e. probable members, while the rest appear to be significantly hotter and possibly compatible with the expected AGN locus.

In conclusion, our 67 sources lacking optical/NIR counterparts are good candidates for new embedded members. Given their absorption, these are rather luminous X-ray sources. They are thus unlikely to be low-mass stars that have escaped optical/NIR detection because intrinsically fainter than the detection limit. Given the small dependency of $L_{\mathrm{X}} / L_{\mathrm{bol}}$ on mass (Flaccomio et al. 2003a; Preibisch et al. 2005), low-mass stars are indeed usually faint in X-rays. Non-identified sources might be embedded protostars (class I and class 0 objects), medium/high-mass very obscured PMS members of NGC 2264 or extragalactic objects shining through the background molecular cloud. They certainly deserve to be followed up with more sensitive IR observations.

\section{Results - X-ray activity}

Our data indicate that the source of X-ray emission in NGC 2264 low-mass members is hot $(0.3-10 \mathrm{keV})$ thermal plasma. The $\mathrm{X}$-ray emission is highly variable in time, the most prominent phenomena being impulsive flares due to magnetic reconnection events. These observations fit well with a solar-like picture of coronal emission and are quite usual for PMS stars. They are, for example, in qualitative agreement with those recently reported for the $\sim 1$ Myr old stars in the ONC by the COUP collaboration (Preibisch et al. 2005). The spectral and temporal characteristics of PMS stars are, broadly speaking, also similar to those of active MS stars, e.g. in the young Pleiades cluster.

The most striking differences with respect to MS stars are the $\mathrm{X}$-ray luminosities and the plasma temperatures, both of which are usually found to be higher. As for $L_{X}$, we note that the fractional X-ray luminosities $\left(L_{\mathrm{X}} / L_{\mathrm{bol}}\right)$ of PMS stars are comparable or smaller than those of saturated MS stars. Therefore, the higher $L_{\mathrm{X}}$ can be explained by the almost saturated emission of PMS stars and by their larger bolometric luminosities. The higher temperatures might instead indicate a difference in the heating mechanism and/or, a larger contribution to the average flux of flares with hard spectra. At least three questions remain open. First and foremost, the nature of the ultimate mechanism that sustains PMS coronae. While for partially convective MS stars this is identified with the $\alpha-\omega$ dynamo thanks to the observed relation between activity and stellar rotation, no such evidence is available for PMS stars (Flaccomio et al. 2003a; Preibisch et al. 2005; Rebull et al. 2006). Second, the extent and geometry of coronae and, in particular, the possibility of interactions of plasma-filled magnetic structures and circumstellar disks (Favata et al. 2005; Jardine et al. 2006). Third, the role of accretion and outflows: soft X-ray emission has been inferred to originate both at the interface between the accretion flow and the photosphere and within the stellar jets (Kastner et al. 2002; Bally et al. 2003). In this section we now use our data to statistically investigate the dependence of activity, in terms of emission level, variability, and spectra, on the stellar and circumstellar characteristics. First, however, we discuss the results of our X-ray spectral analysis in more detail, with respect to plasma temperatures and absorption values.

Figure 15 shows the plasma temperature as a function of absorption for all the sources with spectral fits and for which we adopted isothermal models. Figure 17 similarly shows the two best-fit plasma temperatures of sources for which we adopted $2 \mathrm{~T}$ models. First of all we observe that $2 \mathrm{~T}$ models were required only for $\log N_{\mathrm{H}}<21.5$, probably because higher column densities absorb the cool component to the extent that it becomes 


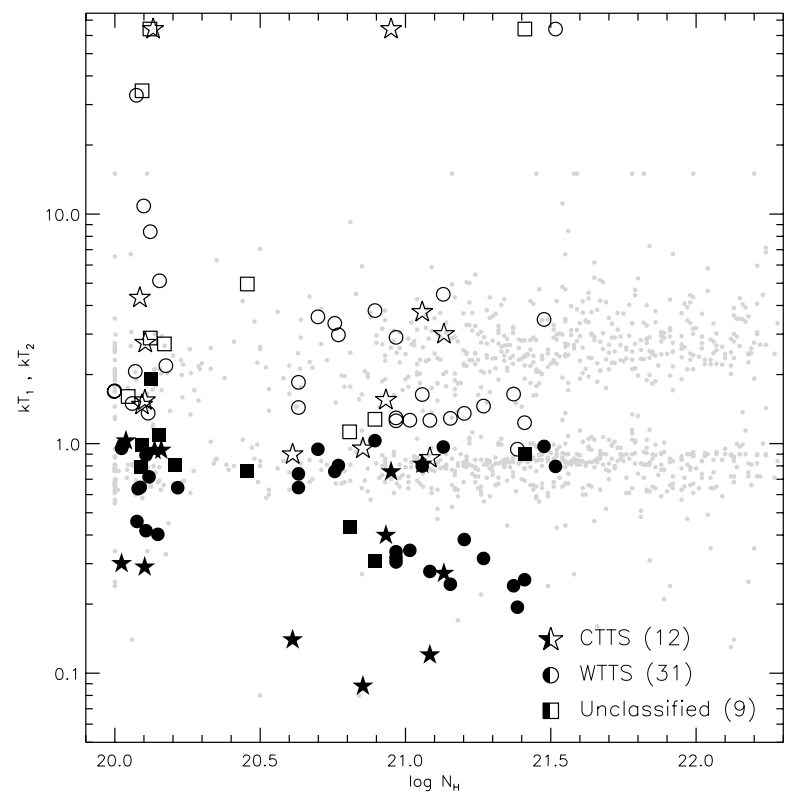

Fig. 17. $k T_{1}$ and $k T_{2}$ as a function of $N_{\mathrm{H}}$ for sources for which $2 \mathrm{~T}$ spectral fits were adopted. The results for NGC 2264 presented in this paper are shown as larger symbols, filled ones for the cool component temperature and empty ones for the hot component. Stars, circles, and squares indicate CTTS, WTTS, and unclassified stars, respectively. The small gray circles show for comparison the two temperatures derived by Getman et al. (2005) for a sample of 566 ONC members observed by the COUP collaboration.

unobservable. Moreover, in the $N_{\mathrm{H}}$ range covered by both models, 2T models were statistically favored in sources with higher statistics while low-statistic sources were in most cases successfully fit with a single plasma component with temperature roughly intermediate between those of the two components in $2 \mathrm{~T}$ models. For $1 \mathrm{~T}$ models we also note a certain correlation between $k T$ and $N_{\mathrm{H}}$ (Fig. 15). While a positive slope of the lower envelope of the datapoints is easily explained as a selection effect, this is not the case for the upper envelope. The paucity of sources with low $N_{\mathrm{H}}$ and high $k T$ indicates that the X-ray emitting plasma of highly extinct sources is intrinsically hotter than that of optically revealed PMS stars. These hot, highly extinct sources are good candidates for embedded Class 0/I protostars, which have already been suggested to have harder X-ray spectra (Imanishi et al. 2001).

Figure 18 shows the run of $k T_{1}$ and $k T_{2}$ with stellar mass for $2 \mathrm{~T}$ models. As for the two previously discussed plots, we also show for reference the temperatures obtained by the COUP collaboration (Preibisch et al. 2005) for $\sim 1$ Myr old stars in the $\mathrm{ONC}^{25}$. With respect to the ONC, temperatures in NGC 2264 appear to be lower on average. This could indicate that at $\sim 3 \mathrm{Myr}$ the hot flaring component of the X-ray emission has become less important. It could also result from a lower fraction of CTTSs given that CTTS tend to have slightly higher plasma temperatures than WTTS (see Sect. 6.1).

We also note that the temperatures of the two isothermal components show a larger scatter in the NGC 2264 stars than in the COUP data. This could be due to: i) larger uncertainties in the NGC 2264 results because of the shorter exposure time and

${ }^{25}$ Note that the COUP observation, obtained with Chandra ACIS, was $850 \mathrm{ks}$ long. The temperatures are thus derived from a spectrum that has been integrated in time over a period that is 8.5 times longer than that of our NGC 2264 observation.

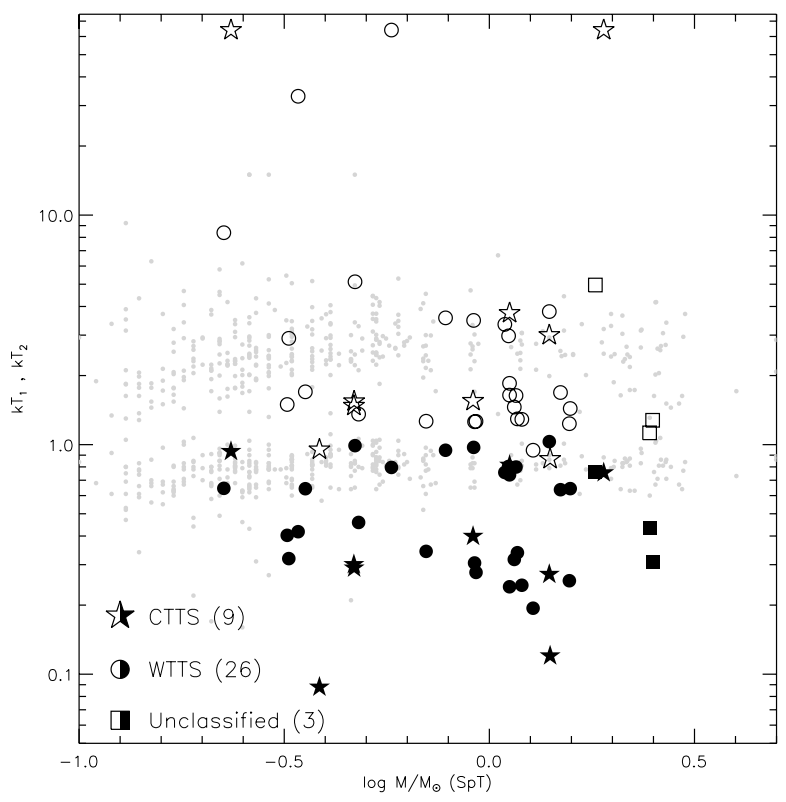

Fig. 18. $k T_{1}$ and $k T_{2}$ as a function of stellar mass for sources that are both placed in the theoretical HR diagram and for which we adopted a 2T spectral model. Symbols and legend as in Fig. 17.

longer distance with respect to the $\mathrm{ONC}$; ii) the shorter exposure time resulting in a stronger influence of spectral time variability on the time-averaged spectra; iii) a real evolutionary effect (e.g. most ONC stars are CTTS, while in NGC 2264 we observe a more varied mixture of CTTS and WTTS); iv) the existence of an additional thermal component that is only sometimes present and/or revealed by the spectral fitting process. In this respect Figs. 17 and 18 indicate, for sources fit with 2T models, an interesting feature: an apparent separation of the temperatures into two branches, more evident for the cool isothermal component. Taking $k T_{1}=0.5 \mathrm{keV}$ as the dividing line between the two branches, we have 23 and 29 sources in the cool and hot branches, respectively. The hot branch has median $k T_{1}$ and $k T_{2}$ of 0.8 and $3.6 \mathrm{keV}$, respectively, roughly coincident with the temperatures of most COUP sources. The cool branch has median $k T_{1}$ and $k T_{2}$ of 0.3 and $1.3 \mathrm{keV}$, respectively. Since this latter median $k T_{2}$ is similar to the temperatures found for sources that were successfully fit by $1 \mathrm{~T}$ models, it is reasonable to hypothesize that the EM distributions of these sources have three peaks: one corresponding to the cool $\sim 0.3 \mathrm{keV}$ component that is not present and/or visible for sources in the hot branch, and two peaks at hotter temperatures (i.e. $\sim 0.8$ and $\sim 3.6 \mathrm{keV}$ ) that are, however, well-represented by an isothermal component with intermediate temperature. Given the limited statistics of our sources, three component spectral models are not needed, although physically reasonable, and would therefore remain unconstrained by the data.

As for the physical origin of the two branches, we note that, with respect to the hot branch, stars in the cool branch have lower counts (median counts 270 vs. 630), are significantly less variable according to the KS test (variability fraction $30 \%$ vs. $66 \%$, at the $1 \%$ confidence level), and are slightly more likely to be CTTS (CTTS fraction: $33 \%$ vs. 23\%). We note that the difference in variability fractions is unlikely to be explained by statistics alone (cf. Figs. 6 and 7). If the $k T_{2} \sim 3 \mathrm{keV}$ component is due to flaring, it is then possible that the $k T_{1} \sim 0.3 \mathrm{keV}$ component becomes detectable preferentially when flaring is absent, so that the hot component is suppressed. This soft emission 
might be physically assimilated to the X-ray emission from the solar corona, which would indeed show temperatures similar to $0.3 \mathrm{keV}$ if analyzed with ASCA SIS (cf. Table 1 in Orlando et al. 2001), an instrument with a response similar to ACIS. The emission measures we derive for the $\sim 0.3 \mathrm{keV}$ component of our NGC 2264 sources $\left(3.1 \times 10^{52}-1.6 \times 10^{54} \mathrm{~cm}^{-3}\right)$, although much larger than those estimated for the Sun, could still be explained by much larger filling factors $(\sim 1)$ and/or larger scale heights of the densest structures (i.e. active region cores).

\subsection{Activity in CTTS and WTTS}

Comparing the plasma temperatures of CTTS and WTTS that are well fit by a single temperature model (37 CTTS, 58 WTTS), we learn that the former are statistically hotter, with a median $k T$ of $1.5 \mathrm{keV}$ vs. $1.3 \mathrm{keV}$ (K-S test probability that the two distributions are compatible: $0.2 \%$ ). A similar comparison for the two plasma temperatures of stars that required a $2 \mathrm{~T}$ spectral model (12 CTTS and 31 WTTS) is inconclusive, possibly because of the lower number of stars. However, we have noted above that CTTS appear to be more common among stars with low values of $k T_{1}$ (Fig. 17). In particular, the three stars with the lowest $k T_{1}$ are all CTTS. Figure 19 shows the ACIS spectra for these three sources, \#17, \#111, and \#183. The two thermal components of the best-fit models are shown separately, and the temperatures and absorptions are reported from Table 6 . The most striking case appears to be source \#183, which has the highest $k T_{1}(0.14 \mathrm{keV})$ and shows a clear double-peaked spectrum. The emission measure $(E M)$ of the cool plasma is estimated to be $5.7 \times 10^{53} \mathrm{~cm}^{-3}$. The other two sources have lower $k T$ and higher EMs (\#17: $k T_{1}=0.09 \mathrm{keV}, E M=2.3 \times 10^{54} \mathrm{~cm}^{-3}$; \#111: $\left.k T_{1}=0.12 \mathrm{keV}, E M=1.2 \times 10^{54} \mathrm{~cm}^{-3}\right)$. We also note that among the three sources \#183 has the largest $\mathrm{H}_{\alpha}$ line equivalent width: 27.9 vs. 10.9 and 18.2 for \#17 and \#111.

As noted in the previous section, low $k T_{1}$ values are usually associated with low $k T_{2}$. For the three CTTS just discussed, for example, the $k T_{2}$ values, $0.87-0.95 \mathrm{keV}$, are among the lowest observed and are very similar to the cool component for the majority of PMS stars. This suggests that the ultra-cold component is present and/or observable with our spectra only when $k T=2-4 \mathrm{kev}$ plasma is absent.

A similar trend can be observed for 1T fits (Fig. 15): among the six lowest $k T$ s $(k T<0.68 \mathrm{keV})$, the $\mathrm{EW}\left(\mathrm{H}_{\alpha}\right)$ is known for three stars, and it indicates accretion (i.e. a CTTS) in all cases. CTTS thus appear to possess both warmer and cooler plasma than WTTS. If this result is confirmed with more statistical significance by further observations, it could imply that the accretion process results, on one hand, in more frequent/energetic flares and, on the other, in a very cool X-ray plasma produced in the accretion shock (cf. the cases of TW Hydrae and BP Tau: Kastner et al. 2002; Stelzer \& Schmitt 2004; Schmitt et al. 2005).

We now investigate the X-ray activity levels $\left(L_{X}\right.$ and $\left.L_{\mathrm{X}} / L_{\mathrm{bol}}\right)$ as a function of bolometric luminosity, stellar mass, and accretion properties for the subsample of stars for which stellar masses were derived by placement in the theoretical HR diagram (Fig. 5) and interpolation of the SDF tracks (160 X-ray detected stars, excluding one possible non-member and two stars outside the tracks). For this investigation we also include upper limits for $16 \mathrm{X}$-ray undetected likely members. Note that a more exhaustive account of the relation between activity and stellar properties, using the X-ray data presented here in conjunction with those of Ramírez et al. (2004a) for another field in NGC 2264 and those of Ramírez et al. (2004b) for the "Orion Flanking Fields", can be found in Rebull et al. (2006). In that
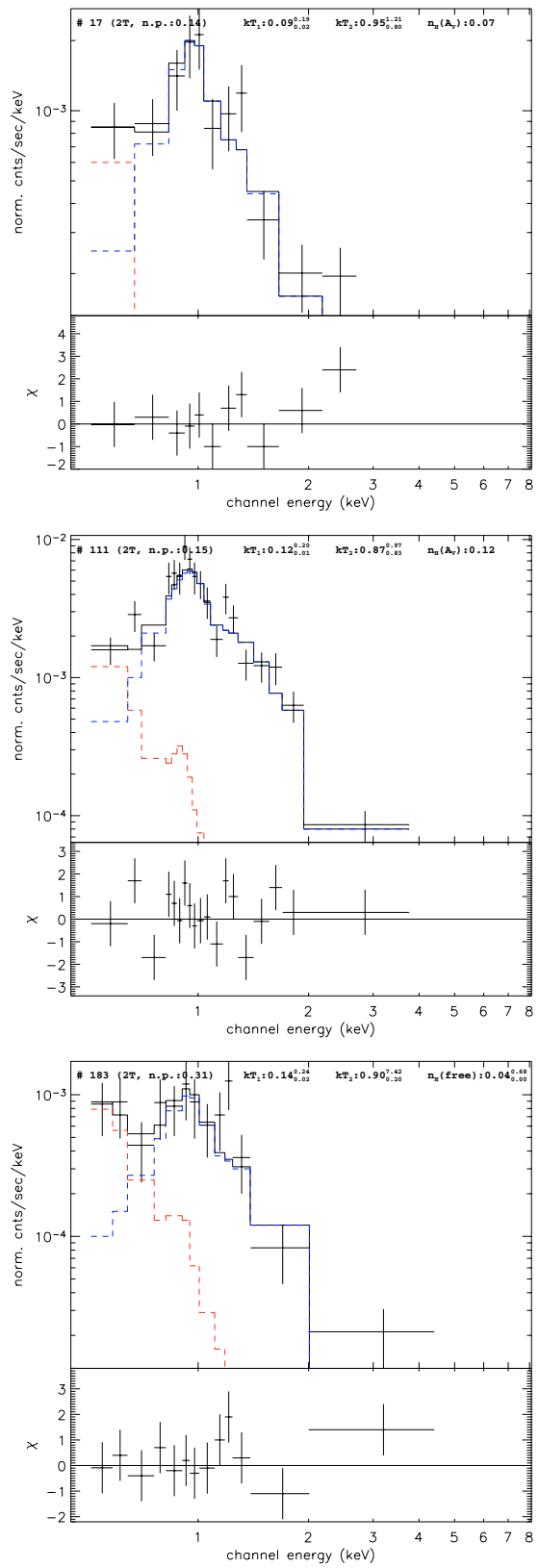

Fig. 19. ACIS spectra of three CTTS with a very low $k T_{1}$. At the top of each panel, we report the source number, fit model, goodness of fit (null probability), $k T_{1}, k T_{2}$, and $N_{\mathrm{H}}$ values, in units of $10^{22} \mathrm{~cm}^{-2}$. For these, we also indicate in parenthesis their origin, " $A_{\mathrm{V}}$ ", or, for source \#183, "free", i.e. derived from the X-ray spectral fitting.

paper the samples for each cluster included many more stars than we have here, but with more uncertain stellar parameters on average. Here we take a different approach, focusing only on the NGC 2264 members in our FOV that are well characterized optically.

Our $L_{X}$-mass scatter plot is shown in Fig. 20. We observe the commonly found mass- $L_{X}$ correlation (e.g. Rebull et al. 2006), although with a large spread. The position of upper limits indicates that our sensitivity $\operatorname{limit}$ is $\log L_{\mathrm{X}} \sim 29 \mathrm{ergs} / \mathrm{s}$, which appears to correspond to a completeness limit in mass at about $0.3-0.4 M_{\odot}$. Moreover we note that, at each stellar mass for which our sample is reasonably complete, CTTS are on average fainter and more scattered with respect to WTTS, confirming the results obtained for ONC stars (Flaccomio et al. 2003a; 


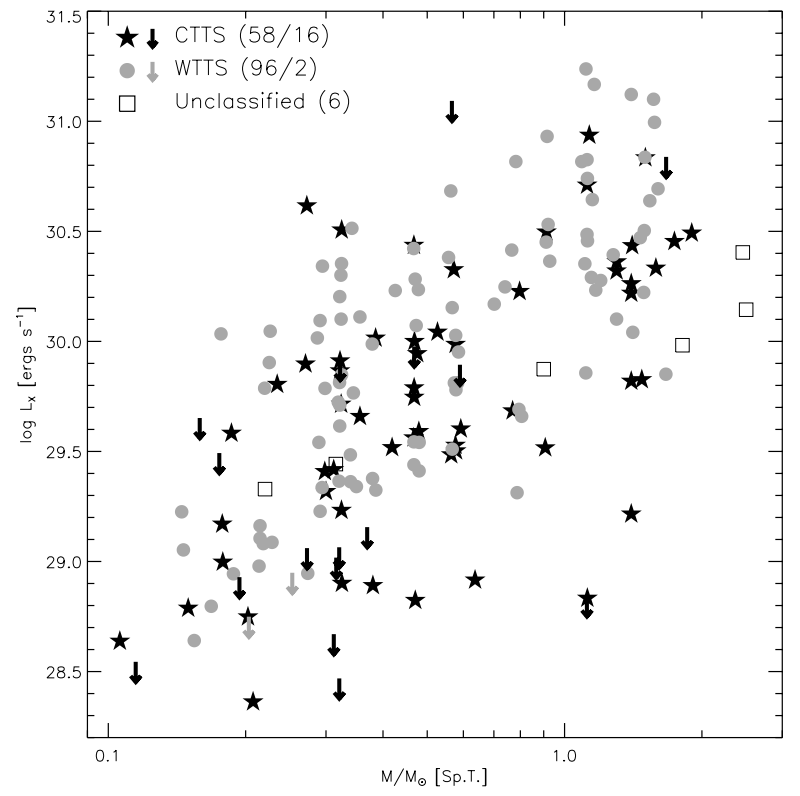

Fig. 20. $\log L_{X}$ vs. mass for likely members placed in the theoretical HR diagram. Black stars and arrows indicate CTTSs (detections and upper limits, respectively). Gray circles and arrows indicate WTTSs. Squares indicate detections of unclassified PMS class. The legend gives the number of detections and upper limits for each of the three subsamples.

Preibisch et al. 2005). A similar plot is shown for $L_{X} / L_{\text {bol }}$ in Fig. 21. $L_{\mathrm{X}} / L_{\text {bol }}$ appears to be generally high, roughly between $10^{-4}$ and the saturation level $\sim 10^{-3.0}$. Twenty-two sources actually have measured $L_{\mathrm{X}} / L_{\mathrm{bol}}$ above the saturation limit. However, a large fraction of these, $73 \%$, are variable, a significantly higher variability fraction than among sources below the saturation threshold (16\%). Moreover, most of the sources with the highest values of $L_{\mathrm{X}} / L_{\mathrm{bol}}$ show large flares, which if excluded would bring them close to or below the saturation level. Flaccomio et al. (2003a) found evidence for ONC stars of a decrease in $L_{\mathrm{X}} / L_{\mathrm{bol}}$ at the very lowest masses. Our sample of stars with mass estimates is not complete enough at those masses to study this effect in detail. However, consistent with these results, we do note a decrease in the upper envelope of the $L_{\mathrm{X}} / L_{\mathrm{bol}}$ vs. mass relation for $M / M_{\odot} \lesssim 0.3$. Considering CTTS and WTTS separately, the difference in activity is less striking in this plot compared to the $L_{X}$-mass one. However, Fig. 22 shows, separately for the two PMS classes, the distributions of $L_{\mathrm{X}} / L_{\mathrm{bol}}$, both for the whole mass range and for the subsample with $M>0.6 M_{\odot}$. As noted above this latter sample should be almost complete, according to the $L_{X}$-mass relation. All the distributions take into account upper limits via the Kaplan-Meier estimator. For both samples, and in particular for the mass-restricted one, we observe that CTTS are less active than WTTS. Median $\log L_{\mathrm{X}} / L_{\text {bol }}$ values differ by 0.32 and 0.41 dex for the two subsamples. The statistical significance of the difference is confirmed by the five two-sample tests in the ASURV package (Feigelson \& Nelson 1985), giving probabilities that the $\log L_{\mathrm{X}} / L_{\text {bol }}$ distributions of CTTS and WTTS are taken from the same parent distribution of $<0.02 \%$ for the whole stellar sample and of $<0.3 \%$ for the mass-restricted one. We note that this latter result differs from that of Preibisch et al. (2005), who find a statistically significant difference in the activity levels of CTTS and WTTS in the ONC only for stars in the $0.2-0.5 M_{\odot}$ mass range.

Finally we used our data to repeat the correlation analysis between $L_{\mathrm{X}}$ and $L_{\text {bol }}$ performed by Preibisch et al. (2005) for ONC

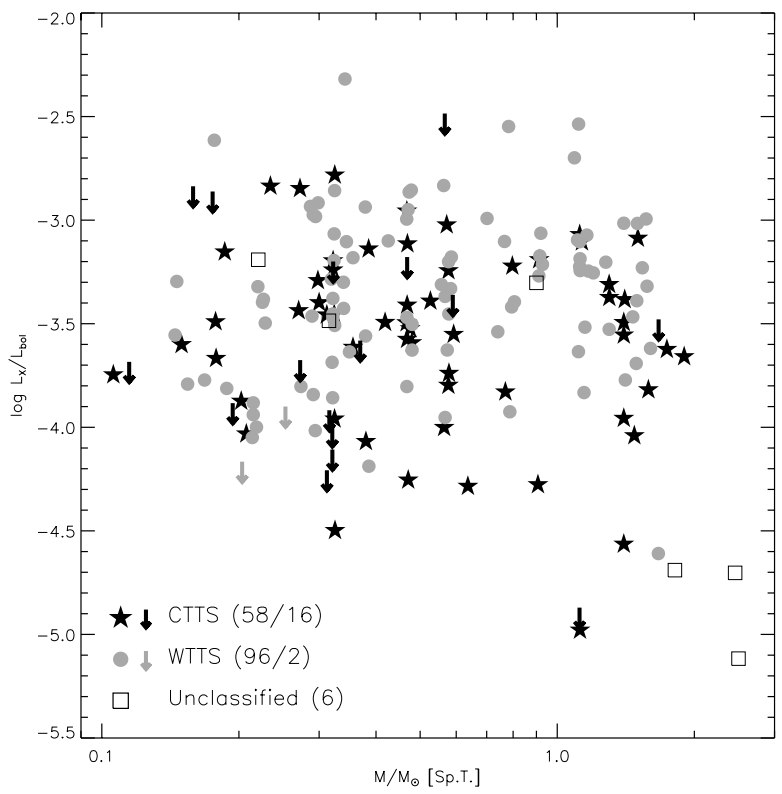

Fig. 21. $\log L_{\mathrm{X}} / L_{\mathrm{bol}}$ vs. mass for the same stars as in Fig. 20.

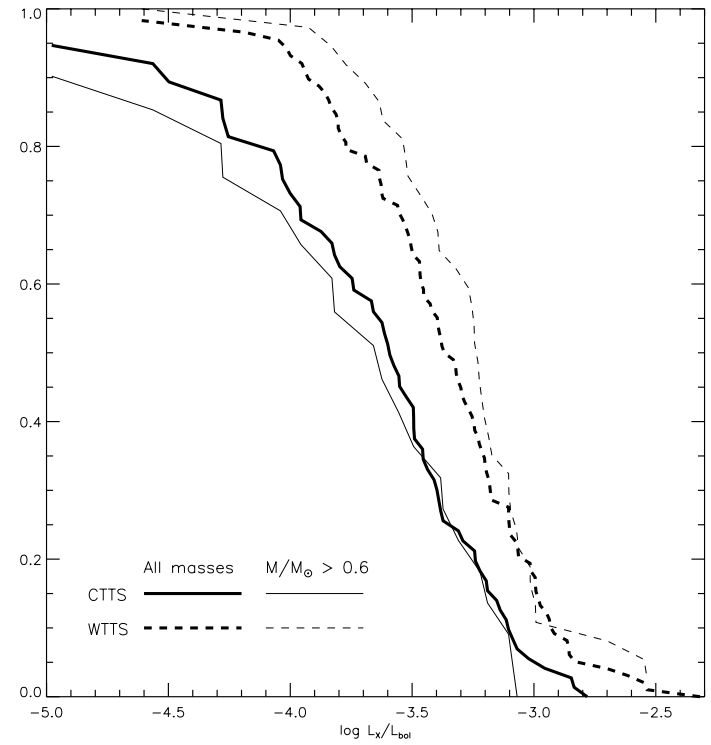

Fig. 22. Distribution of $\log L_{\mathrm{X}} / L_{\mathrm{bol}}$ for WTTS and CTTS (solid and dashed lines, respectively). The thick lines refer to the whole sample of stars depicted in Fig. 21; the thin lines to stars more massive than $0.6 M_{\odot}$. Note how in both cases CTTS are on average less active than WTTS.

stars classified as CTTS and WTTS. Using the estimation maximization (EM) algorithm in the ASURV package, we found very nearly linear correlations between $L_{X}$ and $L_{\text {bol }}$ for the two classes of stars: $\log L_{\mathrm{X}}=(1.02 \pm 0.09) \log L_{\mathrm{bol}}+30.21 \pm 0.05$ for WTTS $(1 \sigma$ dispersion: $0.4 \mathrm{dex})$ and $\log L_{\mathrm{X}}=(1.02 \pm 0.12) \log L_{\mathrm{bol}}+$ $29.92 \pm 0.07$ for CTTS ( $1 \sigma$ dispersion: $0.5 \mathrm{dex})$. As in the ONC case, accreting stars in NGC 2264 thus appear to be fainter on average than non accreting ones with the same $L_{\text {bol }}$ (in this case by a factor of 2) and to have slightly more scattered $L_{X}$ values. However, we note that the power-law slope derived by Preibisch et al. (2005) for accreting stars in the ONC $(0.6 \pm 0.1)$ is significantly shallower than the one derived here and that the $1 \sigma$ dispersion of points with respect to the best-fit relations appear to be larger in the ONC: 0.5 vs. 0.4 dex for WTTS and 0.7 vs. 0.5 
for CTTS. The differences might be interpreted as an evolutionary effect, given that the ONC is younger than NGC 2264 ( 1 vs. $\sim 3 \mathrm{Myr}$ ) and that accretion disks are expected to have evolved significantly in the older cluster (Flaccomio et al. 2003c). We note, however, that the comparison between the effects of accretion on X-ray activity in the two regions is made uncertain by the different accretion indicators used in the two cases, the $\mathrm{H}_{\alpha}$ and the Ca II equivalent widths for NGC 2264 and the ONC, respectively.

\section{2. $K H 15 D$}

The peculiar binary system KH $15 \mathrm{D}$ has been the subject of many investigations. Herbst \& Moran (2005), in particular, analyzed the same X-ray data discussed here to conclude that the system is a very weak source of X-ray emission for its mass and age. They tentatively attribute the low X-ray emission to the high eccentricity of the binary system and/or to the close periastron approach of the two stars, which may either disrupt the stellar magnetosphere and/or adversely affect the stellar dynamo.

The X-ray luminosity we derive for $\mathrm{KH} 15 \mathrm{D}$ (our source \#309), based on 21.6 detected photons, is $1.3 \times 10^{29}$, lower by 0.07 dex than the value reported by Herbst \& Moran (2005). This difference is small and within the uncertainties but, given that the two values are derived from the same X-ray data, we investigated the matter further. This discrepancy can be fully explained by the different estimated number of source photons, 21.6 vs. 22.5 , the assumed value of interstellar absorption, $9 \times 10^{20}$ vs. $2 \times 10^{20} \mathrm{~cm}^{-2}$, and the assumed source spectrum ${ }^{26}$. The 0.07 dex difference in $L_{X}$, however, is not particularly relevant for the physical conclusions regarding the low $L_{X}$ of the system. KH $15 \mathrm{D}$ is not shown in our $L_{X}$-mass scatter plot (Fig. 20) because the system is located in the HR diagram below the grid of the evolutionary tracks (Fig. 5) and we did not derive a mass. By extrapolating the tracks one would estimate a mass of 0.6-0.7 $M_{\odot}$, consistent with the value used by Herbst \& Moran (2005), 0.6 $M_{\odot}$. Thus placing KH $15 \mathrm{D}$ in the $L_{\mathrm{X}}$-mass diagram we notice that it would fall below the bulk of the other NGC 2264 members but in an area that is populated by other CTTSs. The value of $\log L_{\mathrm{X}} / L_{\mathrm{bol}}$ we derive from our data, -3.45 , is moreover perfectly in line with most of the other NGC 2264 members (cf. Fig. 21). We therefore tend to believe that, rather than being affected by the peculiar binary orbits, the low X-ray emission of $\mathrm{KH} 15 \mathrm{D}$ is due to the same mechanism that suppresses activity in CTTSs.

\subsection{Embedded XMM-Newton sources studied by Simon \& Dahm (2005)}

Simon \& Dahm (2005, hereafter SD05) studied three embedded $\mathrm{X}$-ray sources close to IRS 1 in detail (see also Sect. 3.3) using XMM-Newton EPIC data taken in March 2002, i.e. 7 months

\footnotetext{
26 Our $N_{\mathrm{H}}$ is based on $A_{\mathrm{V}}=0.58$, derived here from published photometry and spectral type. Herbst \& Moran (2005) derive their value from $E(B-V)=0.1$. This $E(B-V)$ should, however, imply $N_{\mathrm{H}}=5-6 \times 10^{20} \mathrm{~cm}^{-2}$ assuming $A_{\mathrm{V}} / E(B-V)=3.1$ and depending on the assumed $A_{\mathrm{V}} / N_{\mathrm{H}}$ ratio in the range $1.6-2.0 \times 10^{21}$. Herbst $\&$ Moran (2005) assume solar abundances and $k T=2.7 \mathrm{keV}$, the latter based on the uncertain (due to statistics) hardness ratio. The count to flux conversion factor we used (Eq. (1)) was derived from sources with 50 to 100 counts, which were fit with APEC models with subsolar abundances and median $k T$ of $1.0 \mathrm{keV}$. Note that our harness ratio analysis (cf. Fig. 16) actually indicates a very cool spectrum, $k T \sim 0.3 \mathrm{keV}$, with large uncertainties.
}

before our ACIS exposure. All three sources are retrieved in our data and we now compare the results with respect to spectral characteristics, variability, and average X-ray luminosities.

EPIC source \#26 (our source \#194) was the stablest of the three sources. The lightcurves were constant in both observations; the absorption was identical, $N_{\mathrm{H}}=2.6 \times 10^{22} \mathrm{~cm}^{-2}$; and the $k T$ was also the same within uncertainties ${ }^{27}: 2.09 \pm 0.23 \mathrm{keV}$ (EPIC) vs. $k T=2.4_{1.5}^{4.8} \mathrm{keV}$ (ACIS). The X-ray luminosity, however, when corrected for the different energy band used by SD05 (1-10 keV vs. our 0.5-7 keV) and for the different assumed distance $(d=800 \mathrm{pc}$, vs. our $760 \mathrm{pc})$, seems to have dropped by a factor $\sim 2$ between the two observations.

The EPIC source \#10 (our source \#141) showed a dramatic flare toward the end of SD05's exposure with the peak count rate reaching $\sim 100$ times brighter than the quiescent emission before the flare. Our lightcurve is instead compatible with constant emission. DS05 have analyzed the spectrum during the flare, while we report results for the average spectrum, which is, however, built from only $\sim 65$ photons. There is no evidence of variation in the absorption: DS05 find $N_{\mathrm{H}}=2.26 \pm 0.16 \times 10^{22} \mathrm{~cm}^{-2}$ vs. our $2.6_{1.5}^{4.3} \times 10^{22} \mathrm{~cm}^{-2}$. The temperatures are, however, very different owing to the bright flare in the EPIC data: $k T=$ $12.94 \pm 3.30 \mathrm{keV}$ vs. $k T=2.0_{1.0}^{6.1} \mathrm{keV}$. The X-ray luminosities are also very different, with the SD05 value $\left(L_{X}=32.37\right)$ about 2 dex larger than the value obtained from our analysis and corrected for the different bands and distances. It is, therefore, likely that we observed the source in a state similar to the pre-flare state in the SD05 data.

Finally, the lightcurve of EPIC source \#1 (our source \#296) showed a remarkable rise in count rate during the XMM exposure, by a factor $\geq 7$ in about $8 \mathrm{ks}$, and then began what appears a slow decay for the remaining $30 \mathrm{ks}$ of the observation. An isothermal spectral fit gave a very high absorption, $N_{\mathrm{H}}=$ $9.32 \pm 0.15 \times 10^{22} \mathrm{~cm}^{-2}$, temperature, $k T=10.38 \pm 0.54 \mathrm{keV}$, and total luminosity $L_{\mathrm{X}}=1.1 \times 10^{33} \mathrm{erg} \mathrm{s}^{-1}$ (in the $1-10 \mathrm{keV}$ band). From our ACIS data we derive a two order-of-magnitude lower luminosity $L_{\mathrm{X}}=1.1 \times 10^{31}$, a somewhat colder plasma, $k T=$ $4.8_{3.0}^{9.6} \mathrm{keV}$ and a 5-fold lower absorption $N_{\mathrm{H}}=1.9_{1.6}^{2.4} \times 10^{22} \mathrm{~cm}^{-2}$. The lightcurve indicates a roughly linear decay of the count rate during the $100 \mathrm{ks}$ of our observation from $\sim 6 \mathrm{cts} \mathrm{ks}^{-1}$ to $\sim 2 \mathrm{cts} \mathrm{ks}^{-1}$. We note that our value of $N_{\mathrm{H}}(90 \%$ confidence interval corresponding to $A_{\mathrm{V}}=10-15$ ) agrees roughly with the absorption SD05 derived for the source from NIR spectroscopy $\left(A_{\mathrm{V}}=15-20\right)$ and, contrary to the value observed during the $X M M-N e w t o n$ exposure, does not imply a higher gas-to-dust ratio than in the interstellar medium. We speculate that the high absorption seen by SD05 was due to a solar-like CME associated with the flare as we have also speculated for our source \#71 in Sect. 4.2.

\section{Summary and conclusions}

We observed NGC 2264 with Chandra-ACIS for $97 \mathrm{ks}$, detecting a total of $420 \mathrm{X}$-ray point sources. We identified $85 \%$ of the X-ray sources with known optical and NIR objects, while 67 sources remain with no counterparts in the considered optical/NIR catalogs. More than $90 \%$ of the 353 X-ray sources with counterparts are expected to be members of NGC 2264. Using $\mathrm{H}_{\alpha}$ and optical variability data from the literature we selected a further sample of $83 \mathrm{X}$-ray undetected likely members in the FOV of our ACIS observation, bringing the census of likely

\footnotetext{
27 In this section uncertainties quoted for quantities derived by SD05 are $1 \sigma$, while for our results we quote $90 \%$ confidence intervals.
} 
members with optical/NIR counterparts to more than 400 stars. Taking the small estimated contamination from field stars into account, we have thus increased the known member census of the region by about 100 objects, mostly very low-mass stars, and including some candidate brown dwarfs. A further group of $10 \mathrm{X}$-ray sources, excluded from the member sample because their position in the optical CMD is discrepant with the cluster locus, is also likely to include members. Moreover, among the 67 sources with no optical/NIR counterparts, we argue that about half are previously unrecognized embedded members and good candidates for X-ray detected Class 0/I sources. The other half are instead likely associated with extragalactic objects. The coming SPITZER data will be very useful for clarifying the nature of each source and will allow a systematic study of X-ray activity in the protostellar phase.

We determined X-ray unabsorbed fluxes and luminosities for 326 sources for which absorption could be estimated, either from the X-ray spectra, from optical spectral types and photometry $\left(A_{\mathrm{V}}\right)$, or from NIR photometry. With the aim of shedding light on PMS X-ray activity, we then performed a detailed study of X-ray lightcurves and spectra and studied the relation between the properties of X-ray emitting plasma and stellar/circumstellar characteristics. We confirm several previous findings: $\mathrm{X}$-ray luminosity is related to bolometric luminosity and to stellar mass; $L_{\mathrm{X}} / L_{\mathrm{bol}}$ is on average high and fairly independent of mass, other than for a possible drop at $\sim 2 M_{\odot}$ and a shallow decrease for $M / M_{\odot} \lesssim 0.3$. The mass $-L_{\mathrm{X}}$ relation appears to be better defined for WTTS than for CTTS, and CTTS have on average lower activity levels at any given bolometric luminosity and mass. We found tentative evidence that CTTS are more time variable with respect to WTTS, which might be related to the time-variable nature of the accretion process if it plays a role in the X-ray emission. With respect to spectral characteristics, the plasma on CTTS is on average slightly hotter than on WTTS, a finding possibly related to the higher variability of CTTS. However, we also observe that the sources with the coolest plasma are preferentially CTTS. Three CTTSs in particular appear to have plasma at $\sim 0.1-0.2 \mathrm{keV}$, i.e. comparable to the temperatures expected for plasma heated by accretion shocks, as observed on TW Hydrae $(k T \sim 0.25 \mathrm{keV}$, Kastner et al. 2002; Stelzer \& Schmitt 2004). The estimated emission measures of this cool plasma are between 4 and 17 times larger than on TW Hydrae $\left(E M=1.3 \times 10^{53} \mathrm{~cm}^{-3}\right)$, maybe as a result of the expected higher accretion rates of NGC 2264 stars. These results, taken as a whole, reinforce the mounting evidence that activity in low-mass PMS stars, while generally similar to that of saturated MS stars, is significantly affected by mass accretion. This influence has at least two aspects: accretion is, on one hand, a positive source of very soft $\mathrm{X}$-ray emission produced in the accretion shock. On the other hand, it reduces the average energy output of coronae and makes the emission more time variable. Preibisch et al. (2005) discuss several possible explanations for the suppression of activity. They favor the idea that accretion modifies the magnetic field geometry and results in the "mass-loading" of field lines, thus hampering the heating of plasma to X-ray temperatures. It is at the same time conceivable that the resulting magnetic field structure will be less stable because of the the temporal variability of the mass accretion rate, as well as the rotational shear at the inner edge of the circumstellar disk. To tackle the so-far elusive activity-accretion relation, a better characterization of the circumstellar/accretion properties, e.g. measures of mass accretion rates, is essential. Particularly useful in this respect would be contemporary observations in the X-ray band and in accretion/outflow sensitive optical/NIR lines.

Acknowledgements. The authors wish to thank the anonymous referee for comments that helped to improve this work and acknowledge financial support from the Ministero dell'Istruzione dell'Universitá e della Ricerca, PRIN-INAF and contract ASI-INAF I/023/05/0.

\section{References}

Alexander, D. M., Bauer, F. E., Brandt, W. N., et al. 2003, AJ, 126, 539 Bally, J., Feigelson, E., \& Reipurth, B. 2003, ApJ, 584, 843

Barger, A. J., Cowie, L. L., Capak, P., et al. 2003, AJ, 126, 632

Dahm, S. E., \& Simon, T. 2005, AJ, 129, 829

Damiani, F., Maggio, A., Micela, G., \& Sciortino, S. 1997, ApJ, 483, 350

Damiani, F., \& Micela, G. 1995, ApJ, 446, 341

Favata, F., \& Schmitt, J. H. M. M. 1999, A\&A, 350, 900

Favata, F., Flaccomio, E., Reale, et al., ApJS, 160, 469

Feigelson, E. D., \& Nelson, P. I. 1985, ApJ, 293, 192

Flaccomio, E., Micela, G., Sciortino, S., et al. 2000, A\&A, 355, 651

Flaccomio, E., Damiani, F., Micela, G., et al. 2003a, ApJ, 582, 398

Flaccomio, E., Micela, G., \& Sciortino, S. 2003b, A\&A, 397, 611

Flaccomio, E., Micela, G., \& Sciortino, S. 2003c, A\&A, 402, 277

Flaccomio, E., Micela, G., Sciortino, S., et al. 2005, ApJS, 160, 450

Getman, K. V., Flaccomio, E., Broos, P. S., et al. 2005, ApJS, 160, 319

Glassgold, A. E., Najita, J., \& Igea, J. 2004, ApJ, 615, 972

Haisch, K. E., Jayawardhana, R., \& Alves, J. 2005, ApJ, 627, L57

Hamilton, C. M., Herbst, W., Vrba, F. J., et al. 2005, AJ, 130, 1896

Herbst, W., \& Moran, E. C. 2005, ApJ, 630, 400

Igea, J., \& Glassgold, A. E. 1999, ApJ, 518, 848

Imanishi, K., Koyama, K., \& Tsuboi, Y. 2001, ApJ, 557, 747

Jardine, M., Collier Cameron, A., Donati, J., Gregory, S. G., \& Wood, K. 2006, [arXiv:astro-ph/0601213]

Kastner, J. H., Huenemoerder, D. P., Schulz, N. S., Canizares, C. R., \& Weintraub, D. A. 2002, ApJ, 567, 434

Kenyon, S. J., \& Hartmann, L. 1995, ApJS, 101, 117

Lada, C. J. 2006, [arXiv: astro-ph/0601375]

Lamm, M. H., Bailer-Jones, C. A. L., Mundt, R., Herbst, W., \& Scholz, A. 2004, A\&A, 417, 557

Lorenzani, A., \& Palla, F. 2001, From Darkness to Light: Origin and Evolution of Young Stellar Clusters, ASP Conf. Ser., 243, 745

Luhman, K. L. 1999, ApJ, 525, 466

Margulis, M., Lada, C. J., \& Young, E. T. 1989, ApJ, 345, 906

Meyer, M. R., Calvet, N., \& Hillenbrand, L. A. 1997, AJ, 114, 288

Orlando, S., Peres, G., \& Reale, F. 2001, ApJ, 560, 499

Palla, F., \& Stahler, S. W. 2000, ApJ, 540, 255

Park, B., Sung, H., Bessell, M. S., \& Kang, Y. H. 2000, AJ, 120, 894

Peretto, N., André, P., \& Belloche, A. 2006, A\&A, 445, 979

Pizzolato, N., Maggio, A., Micela, G., Sciortino, S., \& Ventura, P. 2003, A\&A, 397, 147

Preibisch, T., Kim, Y.-C., Favata, Fabio, et al. 2005, ApJS, 160, 401

Ramírez, S. V., Rebull, L., Stauffer, J., et al. 2004a, AJ, 127, 2659

Ramírez, S. V., Rebull, L., Stauffer, J., et al. 2004b, AJ, 128, 787

Rebull, L. M., Makidon, R. B., Strom, S. E., et al. 2002, AJ, 123, 1528

Rebull, L. M., Stauffer, J. R., Ramirez, S. V., et al. 2006, [arXiv: astro-ph/0603027]

Rieke, G. H., \& Lebofsky, M. J. 1985, ApJ, 288, 618

Salpeter, E. E. 1955, ApJ, 121, 16

Schreyer, K., Stecklum, B., Linz, H., \& Henning, T. 2003, ApJ, 599, 335

Schmitt, J. H. M. M., \& Liefke, C. 2004, A\&A, 417, 651

Schmitt, J. H. M. M., Robrade, J., Ness, J.-U., Favata, F., \& Stelzer, B. 2005, A\&A, 432, L35

Siess, L., Dufour, E., \& Forestini, M. 2000, A\&A, 358, 593

Simon, T., \& Dahm, S. E. 2005, ApJ, 618, 795

Stelzer, B., \& Schmitt, J. H. M. M. 2004, A\&A, 418, 687

Sung, H., Bessell, M. S., \& Lee, S.-W. 1997, AJ, 114, 2644

Teixeira, P. S., et al. 2006, [arXiv: astro-ph/0511732]

Vasilevskis, S., Sanders, W. L., Balz, A. G. A. J., 1965, AJ, 70, 797

Vuong, M. H., Montmerle, T., Grosso, et al. 2003, A\&A, 408, 581

Walker, M. F. 1956, ApJS, 2, 365

Williams, J. P., \& Garland, C. A. 2002, ApJ, 568, 259

Wilms, J., Allen, A., \& McCray, R. 2000, ApJ, 542, 914

Young, E. T., et al. 2006, [arXiv: astro-ph/0601300] 


\section{Online Material}


Table 1. Catalog of X-ray ACIS detections.

\begin{tabular}{|c|c|c|c|c|c|c|c|c|c|c|c|c|}
\hline$\overline{\mathrm{N}}$ & $\begin{array}{c}\mathrm{RA} \\
{[\mathrm{h} \mathrm{m} \mathrm{s}]}\end{array}$ & $\begin{array}{c}\text { Dec. } \\
{[\mathrm{d} \mathrm{m} \mathrm{s}] .}\end{array}$ & $\begin{array}{l}\delta_{r, d} \\
{["]} \\
\end{array}$ & $\begin{array}{c}\text { Offax } \\
{[']} \\
\end{array}$ & $\overline{\text { Net Cts. }}$ & $\begin{array}{c}\text { Exp. T. } \\
{[\mathrm{s}]}\end{array}$ & Signif & $\overline{P P_{K S}}$ & $\begin{array}{c}n_{H} \\
{\left[10^{22} \mathrm{~cm}^{-2}\right]} \\
\end{array}$ & $\overline{n_{H}(\mathrm{Ref})}$ & 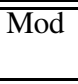 & $\begin{array}{c}F_{X}[\mathrm{u}] \\
{\left[\mathrm{ergs} / \mathrm{s} / \mathrm{cm}^{2}\right]}\end{array}$ \\
\hline$\overline{1} 1$ & $6: 40: 25.8$ & $\overline{99: 29: 24.0}$ & 3.14 & $\overline{9.5}$ & $\overline{25.1}$ & 80579 & $\overline{\overline{6.1}}$ & $\overline{0.088}$ & $\overline{1.59}$ & $\overline{\mathrm{JHK}}$ & $\overline{-}$ & $\overline{-14.03}$ \\
\hline 2 & $6: 40: 27.7$ & 9:32:00.1 & 1.52 & 8.0 & 81.2 & 82888 & 16.2 & 0.262 & 0.00 & Av & $1 \mathrm{~T}$ & -14.35 \\
\hline 3 & $6: 40: 28.1$ & $9: 35: 33.7$ & 1.37 & 7.7 & 44.7 & 81810 & 9.0 & 0.032 & 0.00 & Av & - & -14.52 \\
\hline 4 & $6: 40: 28.6$ & $9: 35: 47.3$ & 1.22 & 7.6 & 186.6 & 64151 & 29.6 & 0.000 & 0.10 & Av & $1 \mathrm{~T}$ & -13.69 \\
\hline 5 & $6: 40: 28.8$ & $9: 30: 59.6$ & 0.59 & 8.1 & 1222.2 & 82889 & 70.2 & 0.000 & 0.26 & $\mathrm{Av}$ & $2 \mathrm{~T}$ & -12.74 \\
\hline 6 & $6: 40: 28.8$ & 9:33:05.3 & 0.80 & 7.5 & 111.0 & 83404 & 17.7 & 0.579 & 0.00 & Av & $1 \mathrm{~T}$ & -14.12 \\
\hline 7 & $6: 40: 30.6$ & $9: 38: 37.7$ & 1.84 & 8.2 & 11.1 & 82255 & 4.7 & 0.724 & - & - & - & - \\
\hline 8 & $6: 40: 30.9$ & $9: 34: 40.4$ & 0.92 & 6.9 & 179.1 & 83958 & 25.7 & 0.001 & 0.62 & $X$ & $1 \mathrm{~T}$ & -13.48 \\
\hline 9 & $6: 40: 31.2$ & $9: 31: 07.0$ & 0.71 & 7.5 & 506.4 & 84042 & 45.7 & 0.093 & 0.09 & Av & $2 \mathrm{~T}$ & -13.31 \\
\hline 10 & $6: 40: 31.7$ & $9: 33: 28.9$ & 1.64 & 6.7 & 18.9 & 84683 & 6.0 & 0.045 & 0.00 & JHK & - & -14.91 \\
\hline 11 & $6: 40: 31.8$ & 9:36:00.6 & 0.90 & 6.9 & 180.4 & 65959 & 27.9 & 0.171 & 0.10 & JHK & $1 \mathrm{~T}$ & -13.75 \\
\hline 12 & $6: 40: 31.9$ & 9:32:16.6 & 1.42 & 6.9 & 35.1 & 84807 & 9.3 & 0.262 & - & - & - & - \\
\hline 13 & $6: 40: 32.9$ & $9: 41: 51.3$ & 2.49 & 9.9 & 81.1 & 74590 & 10.6 & 0.096 & 1.66 & $\mathrm{X}$ & $1 \mathrm{~T}$ & -13.45 \\
\hline 14 & $6: 40: 33.5$ & $9: 34: 58.6$ & 0.86 & 6.3 & 67.9 & 84974 & 14.4 & 0.007 & 0.00 & Av & $1 \mathrm{~T}$ & -14.43 \\
\hline 15 & $6: 40: 33.6$ & $9: 33: 36.2$ & 0.73 & 6.2 & 34.0 & 82415 & 10.0 & 0.327 & 0.00 & JHK & - & -14.64 \\
\hline 16 & $6: 40: 34.3$ & $9: 39: 17.6$ & 1.60 & 7.9 & 26.4 & 82947 & 7.2 & 0.845 & - & - & - & - \\
\hline 17 & $6: 40: 34.5$ & $9: 35: 18.0$ & 0.72 & 6.1 & 140.7 & 84911 & 21.8 & 0.466 & 0.07 & Av & $2 \mathrm{~T}$ & -13.82 \\
\hline 18 & $6: 40: 35.0$ & $9: 39: 54.8$ & 2.17 & 8.2 & 29.0 & 82369 & 7.0 & 0.700 & - & - & - & - \\
\hline 19 & $6: 40: 36.2$ & 9:40:01.6 & 1.60 & 8.0 & 78.3 & 82640 & 14.6 & 0.116 & 0.00 & X & $1 \mathrm{~T}$ & -14.31 \\
\hline 20 & $6: 40: 36.3$ & $9: 28: 04.2$ & 2.17 & 8.3 & 34.8 & 83176 & 7.2 & 0.697 & 0.00 & JHK & - & -14.64 \\
\hline 21 & $6: 40: 36.3$ & $9: 40: 36.9$ & 4.46 & 8.4 & 19.8 & 81817 & 4.9 & 0.320 & - & - & - & - \\
\hline 22 & $6: 40: 36.3$ & 9:43:07.9 & 3.31 & 10.5 & 22.8 & 76153 & 4.8 & 0.189 & - & - & - & - \\
\hline 23 & $6: 40: 36.8$ & $9: 37: 30.4$ & 1.27 & 6.3 & 18.9 & 80653 & 6.0 & 0.836 & - & - & - & - \\
\hline 24 & $6: 40: 36.9$ & $9: 32: 37.5$ & 0.76 & 5.6 & 2.6 & 86958 & 4.9 & 0.829 & - & - & - & - \\
\hline 25 & $6: 40: 37.0$ & 9:39:09.7 & 0.90 & 7.3 & 185.5 & 82420 & 27.3 & 0.000 & 0.14 & $\mathrm{Av}$ & $1 \mathrm{~T}$ & -13.77 \\
\hline 26 & $6: 40: 37.2$ & $9: 31: 09.8$ & 0.65 & 6.1 & 157.2 & 86607 & 26.9 & 0.562 & 0.16 & JHK & $2 \mathrm{~T}$ & -13.80 \\
\hline 27 & $6: 40: 37.7$ & $9: 39: 39.5$ & 1.12 & 7.5 & 86.7 & 81358 & 17.2 & 0.418 & 0.07 & Av & $1 \mathrm{~T}$ & -14.18 \\
\hline 28 & $6: 40: 37.8$ & $9: 41: 23.9$ & 6.32 & 8.8 & 14.1 & 80327 & 5.0 & 0.053 & - & - & - & - \\
\hline 29 & $6: 40: 37.8$ & $9: 35: 56.5$ & 0.43 & 5.4 & 303.4 & 85489 & 32.9 & 0.000 & 0.00 & $X$ & $2 \mathrm{~T}$ & -13.51 \\
\hline 30 & $6: 40: 37.9$ & $9: 34: 54.1$ & 0.34 & 5.2 & 651.1 & 86838 & 54.4 & 0.000 & 0.00 & Av & $1 \mathrm{~T}$ & -13.33 \\
\hline 31 & $6: 40: 38.1$ & $9: 31: 34.6$ & 0.98 & 5.7 & 6.7 & 87213 & 5.4 & 0.277 & - & - & - & - \\
\hline 32 & $6: 40: 38.2$ & $9: 29: 52.5$ & 0.91 & 6.7 & 47.2 & 82997 & 13.0 & 0.376 & 0.06 & Av & - & -14.43 \\
\hline 33 & $6: 40: 38.7$ & $9: 36: 56.9$ & 0.59 & 5.6 & 99.5 & 84957 & 17.0 & 0.989 & 0.00 & Av & $1 \mathrm{~T}$ & -14.22 \\
\hline 34 & $6: 40: 38.7$ & $9: 34: 35.3$ & 0.95 & 5.0 & 13.8 & 87525 & 5.9 & 0.968 & 0.00 & JHK & - & -15.06 \\
\hline 35 & $6: 40: 38.8$ & $9: 29: 48.0$ & 0.88 & 6.6 & 14.5 & 81981 & 6.1 & 0.915 & - & - & - & - \\
\hline 36 & $6: 40: 39.0$ & $9: 35: 59.7$ & 0.67 & 5.2 & 131.6 & 82279 & 25.5 & 0.381 & 0.02 & Av & $1 \mathrm{~T}$ & -14.06 \\
\hline 37 & $6: 40: 39.2$ & $9: 29: 30.4$ & 0.96 & 6.7 & 17.9 & 78920 & 5.8 & 0.133 & - & - & - & - \\
\hline 38 & $6: 40: 39.3$ & $9: 34: 45.9$ & 0.70 & 4.8 & 32.3 & 87604 & 10.4 & 0.228 & 0.40 & $\mathrm{Av}$ & - & -14.32 \\
\hline 39 & $6: 40: 40.1$ & $9: 35: 02.9$ & 0.41 & 4.7 & 256.6 & 87545 & 34.5 & 0.251 & 0.00 & $\mathrm{X}$ & $1 \mathrm{~T}$ & -13.80 \\
\hline 40 & $6: 40: 40.4$ & $9: 31: 34.1$ & 0.72 & 5.2 & 9.7 & 81618 & 6.0 & 0.022 & 0.00 & JHK & - & -15.19 \\
\hline 41 & $6: 40: 40.5$ & $9: 35: 05.5$ & 0.61 & 4.6 & 18.5 & 87679 & 7.4 & 0.586 & - & - & - & - \\
\hline 42 & $6: 40: 40.5$ & $9: 38: 25.6$ & 1.11 & 6.1 & 19.6 & 84592 & 7.8 & 0.190 & - & - & - & - \\
\hline 43 & $6: 40: 40.5$ & $9: 35: 00.5$ & 0.52 & 4.6 & 18.2 & 87764 & 6.6 & 0.988 & - & - & - & - \\
\hline 44 & $6: 40: 40.8$ & $9: 34: 26.4$ & 0.54 & 4.4 & 4.2 & 88477 & 5.4 & 0.514 & 0.00 & JHK & - & -15.59 \\
\hline 45 & $6: 40: 40.9$ & $9: 27: 59.3$ & 0.76 & 7.6 & 120.4 & 84578 & 19.8 & 0.000 & 0.00 & $\mathrm{Av}$ & $1 \mathrm{~T}$ & -14.05 \\
\hline 46 & $6: 40: 41.1$ & $9: 33: 57.6$ & 0.74 & 4.3 & 7.7 & 88601 & 5.4 & 0.372 & 0.00 & JHK & - & -15.32 \\
\hline 47 & $6: 40: 41.2$ & $9: 31: 28.2$ & 0.74 & 5.1 & 7.2 & 84892 & 5.5 & 0.136 & - & - & - & - \\
\hline 48 & $6: 40: 41.3$ & 9:39:09.4 & 1.07 & 6.5 & 30.5 & 80858 & 9.2 & 0.149 & - & - & - & - \\
\hline 49 & $6: 40: 41.6$ & $9: 31: 43.7$ & 0.66 & 4.9 & 18.3 & 84535 & 6.5 & 0.425 & 0.11 & Av & - & -14.79 \\
\hline 50 & $6: 40: 41.8$ & $9: 41: 37.9$ & 2.10 & 8.5 & 37.4 & 81767 & 7.5 & 0.289 & 0.00 & JHK & - & -14.60 \\
\hline 51 & $6: 40: 42.1$ & $9: 31: 42.1$ & 0.75 & 4.8 & 29.5 & 88745 & 9.6 & 0.609 & 0.00 & JHK & - & -14.74 \\
\hline 52 & $6: 40: 42.2$ & $9: 33: 37.5$ & 0.33 & 4.1 & 166.0 & 86733 & 27.1 & 0.145 & 0.00 & $\mathrm{Av}$ & $1 \mathrm{~T}$ & -13.99 \\
\hline 53 & $6: 40: 42.2$ & 9:40:11.1 & 0.74 & 7.2 & 412.4 & 78154 & 42.1 & 0.000 & 0.00 & Av & $1 \mathrm{~T}$ & -13.51 \\
\hline 54 & $6: 40: 42.3$ & 9:39:20.9 & 0.62 & 6.5 & 630.7 & 83614 & 59.7 & 0.000 & 0.00 & JHK & $2 \mathrm{~T}$ & -13.30 \\
\hline 55 & $6: 40: 42.3$ & $9: 34: 25.0$ & 0.51 & 4.1 & 87.5 & 83977 & 22.6 & 0.000 & 0.00 & JHK & $1 \mathrm{~T}$ & -14.28 \\
\hline 56 & $6: 40: 42.4$ & $9: 32: 20.4$ & 0.30 & 4.4 & 257.7 & 87293 & 34.9 & 0.000 & 0.09 & Av & $2 \mathrm{~T}$ & -13.61 \\
\hline 57 & $6: 40: 42.8$ & $9: 33: 35.2$ & 0.45 & 4.0 & 26.6 & 85404 & 8.2 & 0.148 & 0.21 & Av & - & -14.53 \\
\hline 58 & $6: 40: 42.8$ & $9: 39: 05.8$ & 1.26 & 6.2 & 12.4 & 86213 & 5.8 & 0.764 & - & - & - & - \\
\hline 59 & $6: 40: 43.2$ & $9: 31: 14.8$ & 0.39 & 4.9 & 295.0 & 88980 & 40.4 & 0.000 & 0.09 & Av & $2 \mathrm{~T}$ & -13.49 \\
\hline 60 & $6: 40: 43.4$ & $9: 30: 34.3$ & 0.70 & 5.3 & 37.2 & 88511 & 10.8 & 0.136 & 0.29 & Av & - & -14.34 \\
\hline
\end{tabular}


Table 1. (Continued)

\begin{tabular}{|c|c|c|c|c|c|c|c|c|c|c|c|c|}
\hline$\overline{\mathrm{N}}$ & $\begin{array}{c}\text { RA } \\
{[\mathrm{h} \mathrm{m} \mathrm{s}]}\end{array}$ & $\begin{array}{c}\text { Dec. } \\
{[\mathrm{d} \mathrm{m} \mathrm{s}] .}\end{array}$ & $\begin{array}{l}\delta_{r, d} \\
{["]}\end{array}$ & $\begin{array}{c}\text { Offax } \\
\text { ['] }\end{array}$ & Net Cts. & $\begin{array}{c}\text { Exp. T. } \\
{[\mathrm{s}]}\end{array}$ & $\overline{\overline{\text { Signif }}}$ & $P_{K S}$ & $\begin{array}{c}n_{H} \\
{\left[10^{22} \mathrm{~cm}^{-2}\right]}\end{array}$ & 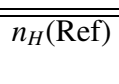 & $\overline{\overline{\text { Mod }}}$ & $\begin{array}{c}F_{X}[\mathrm{u}] \\
{\left[\operatorname{ergs} / \mathrm{s} / \mathrm{cm}^{2}\right]}\end{array}$ \\
\hline$\overline{61}$ & 6 6:40:43.4 & 9:28:40.7 & $\overline{0.92}$ & 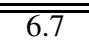 & $\overline{148.9}$ & $\overline{86266}$ & $\overline{26.3}$ & 0.725 & $\overline{0.00}$ & $\overline{\bar{X}}$ & $\overline{1 \mathrm{TT}}$ & -14.03 \\
\hline 62 & $6: 40: 43.6$ & $9: 37: 20.1$ & 0.84 & 4.9 & 17.7 & 82740 & 7.5 & 0.446 & 0.00 & JHK & - & -14.93 \\
\hline 63 & $6: 40: 43.6$ & $9: 31: 14.3$ & 1.04 & 4.8 & 16.5 & 89123 & 5.0 & 0.691 & - & - & _- & - \\
\hline 64 & $6: 40: 43.7$ & $9: 35: 10.2$ & 0.29 & 3.8 & 6.7 & 37810 & 5.3 & 0.276 & 0.00 & JHK & - & -15.01 \\
\hline 65 & $6: 40: 43.7$ & $9: 38: 35.5$ & 0.54 & 5.7 & 326.4 & 87242 & 40.5 & 0.000 & 0.00 & JHK & $2 \mathrm{~T}$ & -13.58 \\
\hline 66 & $6: 40: 43.8$ & $9: 28: 55.2$ & 1.36 & 6.5 & 33.9 & 86733 & 9.7 & 0.345 & 0.00 & JHK & - & -14.67 \\
\hline 67 & $6: 40: 44.2$ & $9: 36: 35.8$ & 0.53 & 4.3 & 12.7 & 85869 & 5.6 & 0.589 & - & - & - & - \\
\hline 68 & $6: 40: 44.6$ & $9: 32: 26.2$ & 0.43 & 3.9 & 65.4 & 90018 & 16.5 & 0.136 & 0.04 & Av & $1 \mathrm{~T}$ & -14.42 \\
\hline 69 & $6: 40: 44.7$ & $9: 34: 04.5$ & 0.41 & 3.5 & 8.3 & 90100 & 5.1 & 0.784 & - & - & - & - \\
\hline 70 & $6: 40: 45.0$ & $9: 38: 49.3$ & 0.86 & 5.7 & 16.4 & 79465 & 7.0 & 0.325 & - & - & - & - \\
\hline 71 & $6: 40: 45.2$ & $9: 28: 44.5$ & 0.58 & 6.4 & 291.4 & 86894 & 34.9 & 0.000 & 1.93 & $\mathrm{X}$ & $1 \mathrm{~T}$ & -13.00 \\
\hline 72 & $6: 40: 45.9$ & $9: 38: 44.3$ & 0.94 & 5.5 & 17.4 & 81482 & 6.1 & 0.585 & 0.00 & JHK & - & -14.93 \\
\hline 73 & $6: 40: 45.9$ & $9: 26: 44.1$ & 2.63 & 8.1 & 9.6 & 80056 & 4.6 & 0.222 & - & - & - & - \\
\hline 74 & $6: 40: 46.7$ & $9: 40: 22.2$ & 1.35 & 6.8 & 38.0 & 73992 & 11.0 & 0.912 & - & - & - & - \\
\hline 75 & $6: 40: 46.9$ & $9: 32: 30.9$ & 0.36 & 3.4 & 95.6 & 90968 & 22.6 & 0.328 & 0.00 & Av & $1 \mathrm{~T}$ & -14.30 \\
\hline 76 & $6: 40: 46.9$ & $9: 33: 01.1$ & 2.38 & 3.2 & 4.0 & 91067 & 5.1 & 0.590 & 1.80 & JHK & - & -14.84 \\
\hline 77 & $6: 40: 47.1$ & $9: 30: 20.0$ & 1.02 & 4.8 & 6.3 & 89567 & 4.7 & 0.394 & 0.00 & JHK & - & -15.41 \\
\hline 78 & $6: 40: 47.1$ & $9: 32: 40.0$ & 0.23 & 3.3 & 571.0 & 91121 & 58.7 & 0.004 & 0.00 & $\mathrm{Av}$ & $2 \mathrm{~T}$ & -13.40 \\
\hline 79 & $6: 40: 47.1$ & $9: 42: 08.5$ & 1.89 & 8.4 & 15.1 & 74097 & 5.0 & 0.919 & 0.01 & $\mathrm{Av}$ & - & -14.95 \\
\hline 80 & $6: 40: 47.2$ & $9: 31: 08.9$ & 0.64 & 4.2 & 13.3 & 90300 & 6.7 & 0.494 & 0.00 & $\mathrm{Av}$ & - & -15.09 \\
\hline 81 & $6: 40: 47.6$ & $9: 40: 33.5$ & 1.35 & 6.9 & 15.8 & 82215 & 5.2 & 0.233 & 0.00 & JHK & - & -14.97 \\
\hline 82 & $6: 40: 47.8$ & $9: 26: 45.1$ & 1.31 & 8.0 & 47.1 & 84535 & 11.2 & 0.581 & - & - & - & - \\
\hline 83 & $6: 40: 47.9$ & 9:33:03.0 & 0.27 & 2.9 & 98.4 & 91483 & 20.6 & 0.013 & 0.00 & JHK & $1 \mathrm{~T}$ & -14.16 \\
\hline 84 & $6: 40: 48.0$ & $9: 32: 03.2$ & 0.57 & 3.4 & 6.9 & 91185 & 4.7 & 0.682 & 0.00 & JHK & - & -15.38 \\
\hline 85 & $6: 40: 48.1$ & $9: 34: 05.4$ & 0.56 & 2.6 & 10.8 & 91488 & 5.1 & 0.037 & 0.00 & JHK & _- & -15.19 \\
\hline 86 & $6: 40: 48.1$ & $9: 27: 01.1$ & 1.48 & 7.7 & 26.9 & 84955 & 8.1 & 0.330 & 0.00 & $\mathrm{Av}$ & _- & -14.76 \\
\hline 87 & $6: 40: 48.3$ & $9: 36: 38.5$ & 0.23 & 3.5 & 538.7 & 87849 & 54.9 & 0.000 & 0.00 & $\mathrm{X}$ & $1 \mathrm{~T}$ & -13.42 \\
\hline 88 & $6: 40: 48.5$ & $9: 34: 16.6$ & 0.61 & 2.5 & 15.4 & 91546 & 7.4 & 0.875 & 0.00 & JHK & - & -15.03 \\
\hline 89 & $6: 40: 48.6$ & $9: 35: 57.4$ & 0.24 & 3.0 & 150.3 & 92107 & 25.8 & 0.232 & 1.65 & JHK & $1 \mathrm{~T}$ & -13.35 \\
\hline 90 & $6: 40: 48.6$ & $9: 32: 52.3$ & 0.21 & 2.8 & 246.1 & 91775 & 33.8 & 0.000 & 0.10 & Av & $2 \mathrm{~T}$ & -13.67 \\
\hline 91 & $6: 40: 48.8$ & $9: 32: 42.5$ & 0.23 & 2.9 & 165.9 & 91786 & 28.7 & 0.135 & 0.27 & Av & $1 \mathrm{~T}$ & -13.74 \\
\hline 92 & $6: 40: 49.0$ & $9: 36: 55.1$ & 0.99 & 3.6 & 7.9 & 91255 & 4.9 & 0.426 & - & - & - & - \\
\hline 93 & $6: 40: 49.1$ & $9: 37: 36.2$ & 0.57 & 4.1 & 27.3 & 86242 & 9.3 & 0.740 & 0.17 & JHK & - & -14.55 \\
\hline 94 & $6: 40: 49.5$ & $9: 31: 41.5$ & 0.77 & 3.4 & 16.9 & 84088 & 8.9 & 0.554 & - & - & - & - \\
\hline 95 & $6: 40: 49.9$ & $9: 36: 49.2$ & 0.35 & 3.4 & 114.7 & 91665 & 23.9 & 0.776 & 0.19 & Av & $1 \mathrm{~T}$ & -14.02 \\
\hline 96 & $6: 40: 50.1$ & $9: 29: 32.4$ & 1.63 & 5.2 & 14.2 & 89256 & 6.1 & 0.272 & - & - & - & - \\
\hline 97 & $6: 40: 50.1$ & $9: 30: 39.7$ & 0.36 & 4.2 & 121.1 & 90500 & 23.5 & 0.000 & 0.22 & $X$ & $1 \mathrm{~T}$ & -13.81 \\
\hline 98 & $6: 40: 50.5$ & 9:32:19.6 & 0.27 & 2.8 & 100.1 & 88572 & 20.8 & 0.297 & 2.68 & $X$ & $1 \mathrm{~T}$ & -13.43 \\
\hline 99 & $6: 40: 50.6$ & $9: 31: 31.1$ & 0.51 & 3.4 & 8.9 & 91357 & 6.0 & 0.842 & 0.00 & JHK & - & -15.27 \\
\hline 100 & $6: 40: 50.8$ & $9: 41: 55.9$ & 1.20 & 7.9 & 27.5 & 83075 & 7.4 & 0.718 & 0.00 & JHK & - & -14.74 \\
\hline 101 & $6: 40: 51.0$ & $9: 38: 52.8$ & 0.74 & 5.0 & 38.6 & 88762 & 10.0 & 0.532 & - & - & - & - \\
\hline 102 & $6: 40: 51.3$ & $9: 43: 19.2$ & 2.24 & 9.3 & 88.2 & 76964 & 5.5 & 0.034 & 0.09 & Av & $1 \mathrm{~T}$ & -14.01 \\
\hline 103 & $6: 40: 51.4$ & $9: 30: 13.4$ & 0.58 & 4.4 & 22.6 & 90340 & 8.5 & 0.160 & 0.00 & Av & - & -14.86 \\
\hline 104 & $6: 40: 51.5$ & $9: 37: 14.2$ & 0.38 & 3.5 & 78.5 & 91576 & 20.2 & 0.026 & 0.00 & Av & $2 \mathrm{~T}$ & -14.30 \\
\hline 105 & $6: 40: 51.6$ & $9: 28: 44.5$ & 0.57 & 5.8 & 267.5 & 88272 & 35.7 & 0.189 & 0.23 & Av & $1 \mathrm{~T}$ & -13.52 \\
\hline 106 & $6: 40: 52.1$ & $9: 29: 13.8$ & 0.53 & 5.3 & 105.6 & 89254 & 21.2 & 0.280 & 0.00 & $\mathrm{Av}$ & $2 \mathrm{~T}$ & -14.03 \\
\hline 107 & $6: 40: 52.6$ & $9: 29: 53.4$ & 0.51 & 4.6 & 113.6 & 90240 & 22.4 & 0.423 & 2.86 & $\mathrm{X}$ & $1 \mathrm{~T}$ & -13.40 \\
\hline 108 & $6: 40: 52.9$ & $9: 35: 15.2$ & 0.25 & 1.8 & 107.2 & 90348 & 23.8 & 0.031 & 2.80 & $\mathrm{X}$ & $1 \mathrm{~T}$ & -13.33 \\
\hline 109 & $6: 40: 53.2$ & $9: 29: 54.3$ & 0.67 & 4.5 & 6.7 & 90369 & 5.8 & 0.070 & 0.00 & JHK & - & -15.39 \\
\hline 110 & $6: 40: 53.6$ & $9: 36: 45.6$ & 0.47 & 2.8 & 9.6 & 87693 & 6.4 & 0.617 & 0.00 & JHK & - & -15.22 \\
\hline 111 & $6: 40: 53.6$ & $9: 33: 24.6$ & 0.18 & 1.5 & 365.2 & 93281 & 45.8 & 0.016 & 0.12 & Av & $2 \mathrm{~T}$ & -13.40 \\
\hline 112 & $6: 40: 53.8$ & $9: 30: 38.8$ & 0.30 & 3.8 & 195.2 & 91356 & 32.2 & 0.777 & 0.03 & Av & $2 \mathrm{~T}$ & -13.86 \\
\hline 113 & $6: 40: 53.9$ & $9: 38: 04.8$ & 0.58 & 4.0 & 1.7 & 90692 & 5.2 & 0.002 & - & - & - & - \\
\hline 114 & $6: 40: 54.1$ & $9: 29: 51.0$ & 0.31 & 4.5 & 726.9 & 90475 & 66.7 & 0.000 & 0.00 & JHK & $2 \mathrm{~T}$ & -13.28 \\
\hline 115 & $6: 40: 54.7$ & $9: 31: 58.9$ & 0.55 & 2.5 & 6.1 & 93115 & 4.8 & 0.027 & - & - & - & - \\
\hline 116 & $6: 40: 55.2$ & $9: 33: 18.6$ & 0.45 & 1.3 & 10.7 & 93618 & 6.8 & 0.790 & 0.00 & $\mathrm{Av}$ & - & -15.20 \\
\hline 117 & $6: 40: 55.2$ & $9: 30: 16.6$ & 0.69 & 4.1 & 7.8 & 91188 & 5.4 & 0.070 & - & - & - & - \\
\hline 118 & $6: 40: 55.3$ & $9: 33: 24.6$ & 0.32 & 1.2 & 18.5 & 93618 & 8.2 & 0.007 & 0.00 & JHK & - & -14.96 \\
\hline 119 & $6: 40: 55.3$ & $9: 39: 59.0$ & 0.72 & 5.8 & 110.1 & 87488 & 21.5 & 0.040 & 0.20 & Av & $1 \mathrm{~T}$ & -13.97 \\
\hline 120 & $6: 40: 55.4$ & $9: 37: 23.4$ & 0.26 & 3.3 & 362.5 & 92186 & 44.2 & 0.170 & 0.12 & Av & $2 \mathrm{~T}$ & -13.48 \\
\hline
\end{tabular}


Table 1. (Continued)

\begin{tabular}{|c|c|c|c|c|c|c|c|c|c|c|c|c|}
\hline $\mathrm{N}$ & $\begin{array}{c}\text { RA } \\
{[\mathrm{h} \mathrm{m} \mathrm{s}]}\end{array}$ & $\begin{array}{c}\text { Dec. } \\
{[\mathrm{d} \mathrm{m} \mathrm{s}] .}\end{array}$ & $\begin{array}{l}\delta_{r, d} \\
{["]}\end{array}$ & $\begin{array}{c}\text { Offax } \\
\text { ['] }\end{array}$ & Net Cts. & $\begin{array}{c}\text { Exp. T. } \\
{[\mathrm{s}]}\end{array}$ & Signif & $P_{K S}$ & $\begin{array}{c}n_{H} \\
{\left[10^{22} \mathrm{~cm}^{-2}\right]}\end{array}$ & $\overline{\overline{n_{H}}(\text { Ref })}$ & $\overline{\text { Mod }}$ & $\begin{array}{c}F_{X}[\mathrm{u}] \\
{\left[\operatorname{ergs} / \mathrm{s} / \mathrm{cm}^{2}\right]}\end{array}$ \\
\hline$\overline{121}$ & $6: 40: 55.5$ & $99: 31: 21.8$ & 0.57 & $\overline{\overline{3.0}}$ & $\overline{14.2}$ & 92455 & $\overline{\overline{8.2}}$ & 0.773 & $\overline{\overline{0.00}}$ & $\overline{\overline{\text { JHK }}}$ & 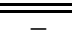 & -15.07 \\
\hline 122 & $6: 40: 55.5$ & $9: 38: 38.7$ & 0.92 & 4.5 & 11.9 & 86476 & 6.0 & 0.000 & 0.00 & JHK & - & -15.12 \\
\hline 123 & $6: 40: 55.6$ & $9: 38: 24.5$ & 0.48 & 4.2 & 19.8 & 86203 & 6.8 & 0.144 & 0.02 & $\mathrm{Av}$ & - & -14.89 \\
\hline 124 & $6: 40: 55.8$ & $9: 40: 18.0$ & 0.52 & 6.1 & 426.1 & 86940 & 42.9 & 0.000 & 0.00 & $\mathrm{Av}$ & $2 \mathrm{~T}$ & -13.33 \\
\hline 125 & $6: 40: 55.8$ & $9: 36: 05.8$ & 0.48 & 2.0 & 8.2 & 94475 & 6.5 & 0.047 & - & - & - & - \\
\hline 126 & $6: 40: 55.9$ & $9: 37: 17.4$ & 0.36 & 3.1 & 35.9 & 92447 & 11.2 & 0.370 & 0.00 & $\mathrm{Av}$ & - & -14.67 \\
\hline 127 & $6: 40: 56.2$ & $9: 36: 30.7$ & 0.22 & 2.4 & 207.3 & 93839 & 32.1 & 0.040 & 0.90 & $X$ & $1 \mathrm{~T}$ & -13.39 \\
\hline 128 & $6: 40: 56.2$ & $9: 40: 37.6$ & 1.35 & 6.4 & 9.0 & 86359 & 5.0 & 0.905 & 0.00 & JHK & - & -15.24 \\
\hline 129 & $6: 40: 56.3$ & $9: 39: 33.1$ & 2.22 & 5.3 & 12.1 & 84741 & 6.0 & 0.850 & 0.07 & Av & - & -15.02 \\
\hline 130 & $6: 40: 56.3$ & $9: 34: 17.9$ & 0.30 & 0.6 & 49.5 & 93507 & 16.9 & 0.985 & 0.00 & JHK & - & -14.53 \\
\hline 131 & $6: 40: 56.4$ & $9: 35: 53.1$ & 0.42 & 1.7 & 16.4 & 93022 & 8.5 & 0.101 & 0.44 & Av & - & -14.62 \\
\hline 132 & $6: 40: 56.5$ & $9: 29: 53.6$ & 0.69 & 4.4 & 30.3 & 90643 & 11.4 & 0.895 & 0.00 & $\mathrm{Av}$ & - & -14.73 \\
\hline 133 & $6: 40: 56.8$ & $9: 30: 14.8$ & 0.42 & 4.0 & 194.5 & 91106 & 32.9 & 0.006 & 0.00 & $X$ & $1 \mathrm{~T}$ & -13.91 \\
\hline 134 & $6: 40: 56.8$ & $9: 29: 59.4$ & 0.64 & 4.3 & 14.4 & 75335 & 6.8 & 0.625 & 0.11 & $\mathrm{Av}$ & - & -14.84 \\
\hline 135 & $6: 40: 56.8$ & $9: 37: 48.9$ & 0.21 & 3.6 & 839.4 & 91676 & 66.2 & 0.000 & 0.11 & $\mathrm{Av}$ & $2 \mathrm{~T}$ & -13.13 \\
\hline 136 & $6: 40: 57.0$ & $9: 33: 01.2$ & 0.17 & 1.3 & 150.3 & 93963 & 26.3 & 0.907 & 0.29 & $\mathrm{Av}$ & $1 \mathrm{~T}$ & -13.82 \\
\hline 137 & $6: 40: 57.5$ & $9: 29: 23.4$ & 0.89 & 4.9 & 42.0 & 37661 & 13.5 & 0.481 & 0.00 & JHK & - & -14.21 \\
\hline 138 & $6: 40: 57.5$ & $9: 26: 16.6$ & 1.64 & 8.0 & 15.2 & 82088 & 6.4 & 0.563 & 0.00 & JHK & - & -14.99 \\
\hline 139 & $6: 40: 57.6$ & $9: 37: 07.1$ & 0.29 & 2.9 & 14.7 & 91165 & 6.0 & 0.876 & 0.00 & $\mathrm{Av}$ & - & -15.05 \\
\hline 140 & $6: 40: 57.7$ & $9: 31: 50.0$ & 0.29 & 2.4 & 21.6 & 93553 & 9.2 & 0.129 & 0.00 & $\mathrm{Av}$ & - & -14.90 \\
\hline 141 & $6: 40: 57.7$ & $9: 36: 08.0$ & 0.27 & 1.9 & 70.6 & 90901 & 19.7 & 0.145 & 2.62 & $\mathrm{X}$ & $1 \mathrm{~T}$ & -13.44 \\
\hline 142 & $6: 40: 57.8$ & $9: 30: 50.3$ & 0.22 & 3.4 & 244.2 & 55899 & 31.1 & 0.444 & 0.21 & $\mathrm{Av}$ & $1 \mathrm{~T}$ & -13.42 \\
\hline 143 & $6: 40: 57.9$ & $9: 41: 20.0$ & 1.71 & 7.1 & 48.5 & 80975 & 11.6 & 0.923 & 0.10 & $\mathrm{Av}$ & - & -14.35 \\
\hline 144 & $6: 40: 57.9$ & $9: 27: 09.0$ & 1.70 & 7.1 & 13.3 & 84375 & 5.4 & 0.146 & - & - & - & - \\
\hline 145 & $6: 40: 58.0$ & $9: 36: 39.2$ & 0.55 & 2.4 & 11.8 & 89724 & 5.4 & 0.192 & - & - & - & - \\
\hline 146 & $6: 40: 58.1$ & $9: 36: 52.8$ & 0.31 & 2.6 & 26.2 & 89301 & 8.7 & 0.258 & 0.33 & JHK & - & -14.46 \\
\hline 147 & $6: 40: 58.3$ & $9: 37: 56.6$ & 0.36 & 3.7 & 34.9 & 89715 & 10.3 & 0.395 & 0.00 & JHK & _- & -14.67 \\
\hline 148 & $6: 40: 58.4$ & $9: 27: 24.9$ & 1.20 & 6.8 & 51.7 & 85243 & 13.4 & 0.049 & 0.00 & JHK & - & -14.48 \\
\hline 149 & $6: 40: 58.5$ & $9: 33: 31.6$ & 0.10 & 0.7 & 3194.7 & 94186 & 147.2 & 0.000 & 0.00 & JHK & $2 \mathrm{~T}$ & -12.55 \\
\hline 150 & $6: 40: 58.6$ & $9: 36: 39.2$ & 0.49 & 2.4 & 7.3 & 90740 & 6.0 & 0.360 & - & - & - & - \\
\hline 151 & $6: 40: 58.7$ & $9: 36: 13.0$ & 0.15 & 2.0 & 781.5 & 94749 & 66.1 & 0.000 & 0.04 & $\mathrm{Av}$ & $1 \mathrm{~T}$ & -13.22 \\
\hline 152 & $6: 40: 58.8$ & $9: 34: 05.3$ & 0.41 & 0.1 & 16.3 & 94144 & 8.9 & 0.078 & - & - & - & - \\
\hline 153 & $6: 40: 58.8$ & $9: 42: 24.6$ & 1.49 & 8.2 & 10.0 & 78820 & 4.7 & 0.951 & 0.00 & JHK & - & -15.16 \\
\hline 154 & $6: 40: 58.8$ & $9: 30: 57.1$ & 0.23 & 3.3 & 205.4 & 43709 & 27.2 & 0.732 & 0.09 & $\mathrm{Av}$ & $2 \mathrm{~T}$ & -13.35 \\
\hline 155 & $6: 40: 58.8$ & $9: 39: 18.4$ & 0.46 & 5.1 & 202.2 & 86462 & 28.1 & 0.055 & 0.00 & $X$ & $1 \mathrm{~T}$ & -13.85 \\
\hline 156 & $6: 40: 58.9$ & $9: 36: 46.0$ & 0.36 & 2.5 & 28.8 & 89311 & 11.2 & 0.234 & - & - & - & - \\
\hline 157 & $6: 40: 58.9$ & $9: 28: 52.8$ & 0.62 & 5.4 & 43.8 & 88230 & 14.1 & 0.224 & 0.07 & JHK & - & -14.47 \\
\hline 158 & $6: 40: 59.1$ & $9: 33: 22.2$ & 0.97 & 0.9 & 8.1 & 94226 & 4.8 & 0.309 & - & - & - & - \\
\hline 159 & $6: 40: 59.2$ & $9: 31: 50.4$ & 0.34 & 2.4 & 14.1 & 40232 & 6.6 & 0.954 & 0.67 & JHK & - & -14.22 \\
\hline 160 & $6: 40: 59.3$ & $9: 33: 25.5$ & 0.50 & 0.8 & 7.9 & 94258 & 5.6 & 0.501 & - & - & - & - \\
\hline 161 & $6: 40: 59.3$ & $9: 35: 52.2$ & 0.22 & 1.6 & 161.4 & 95208 & 30.4 & 0.772 & 2.37 & JHK & $1 \mathrm{~T}$ & -13.29 \\
\hline 162 & $6: 40: 59.3$ & $9: 35: 56.8$ & 0.28 & 1.7 & 63.1 & 95135 & 18.1 & 0.362 & 2.73 & $X$ & $1 \mathrm{~T}$ & -13.39 \\
\hline 163 & $6: 40: 59.4$ & $9: 33: 33.2$ & 0.16 & 0.7 & 205.9 & 94283 & 31.5 & 0.062 & 0.30 & JHK & $1 \mathrm{~T}$ & -13.65 \\
\hline 164 & $6: 40: 59.5$ & $9: 29: 51.5$ & 0.49 & 4.4 & 40.1 & 89447 & 11.9 & 0.058 & 0.00 & $\mathrm{Av}$ & - & -14.61 \\
\hline 165 & $6: 40: 59.5$ & $9: 36: 10.2$ & 0.24 & 1.9 & 127.1 & 94907 & 26.6 & 0.157 & 3.02 & $\mathrm{X}$ & $1 \mathrm{~T}$ & -13.15 \\
\hline 166 & $6: 40: 59.5$ & $9: 39: 06.2$ & 0.57 & 4.9 & 10.8 & 80058 & 5.1 & 0.739 & 0.19 & JHK & - & -14.91 \\
\hline 167 & $6: 40: 59.5$ & $9: 35: 10.7$ & 0.14 & 1.0 & 1067.6 & 95643 & 83.0 & 0.486 & 0.30 & $\mathrm{Av}$ & $2 \mathrm{~T}$ & -12.91 \\
\hline 168 & $6: 40: 59.5$ & $9: 35: 51.7$ & 0.45 & 1.6 & 11.1 & 95228 & 7.1 & 0.297 & - & - & - & - \\
\hline 169 & $6: 40: 59.6$ & $9: 36: 57.5$ & 0.50 & 2.7 & 10.0 & 93518 & 5.9 & 0.296 & 0.01 & Av & - & -15.23 \\
\hline 170 & $6: 40: 59.7$ & $9: 28: 43.3$ & 0.84 & 5.5 & 23.1 & 89213 & 7.9 & 0.370 & 0.22 & JHK & - & -14.60 \\
\hline 171 & $6: 40: 60.0$ & 9:35:00.7 & 0.28 & 0.8 & 4.0 & 91008 & 5.0 & 0.419 & - & - & - & - \\
\hline 172 & $6: 40: 60.0$ & $9: 28: 50.1$ & 0.30 & 5.4 & 1122.7 & 86974 & 69.8 & 0.000 & 0.05 & $\mathrm{Av}$ & $2 \mathrm{~T}$ & -13.02 \\
\hline 173 & $6: 41: 00.2$ & $9: 36: 30.9$ & 0.67 & 2.3 & 9.2 & 94348 & 5.4 & 0.416 & 2.17 & JHK & - & -14.44 \\
\hline 174 & $6: 41: 00.3$ & 9:35:58.9 & 0.33 & 1.8 & 39.3 & 95166 & 13.0 & 0.337 & - & - & - & - \\
\hline 175 & $6: 41: 00.4$ & $9: 41: 15.5$ & 0.99 & 7.0 & 9.2 & 83011 & 5.3 & 0.660 & 0.00 & JHK & - & -15.21 \\
\hline 176 & $6: 41: 00.5$ & $9: 29: 15.9$ & 0.30 & 5.0 & 879.5 & 88795 & 66.9 & 0.122 & 0.23 & $X$ & $1 \mathrm{~T}$ & -13.00 \\
\hline 177 & $6: 41: 00.5$ & $9: 36: 12.2$ & 0.38 & 2.0 & 27.1 & 94913 & 10.5 & 0.489 & 1.41 & JHK & - & -14.11 \\
\hline 178 & $6: 41: 00.6$ & 9:36:09.9 & 0.25 & 2.0 & 115.9 & 94978 & 24.5 & 0.237 & 0.35 & $X$ & $1 \mathrm{~T}$ & -13.75 \\
\hline 179 & $6: 41: 01.0$ & $9: 32: 44.4$ & 0.15 & 1.6 & 835.4 & 95245 & 73.3 & 0.000 & 0.13 & JHK & $2 \mathrm{~T}$ & -13.10 \\
\hline 180 & $6: 41: 01.0$ & $9: 29: 37.4$ & 0.59 & 4.6 & 4.4 & 89533 & 4.8 & 0.200 & - & - & - & - \\
\hline
\end{tabular}


Table 1. (Continued)

\begin{tabular}{|c|c|c|c|c|c|c|c|c|c|c|c|c|}
\hline$\overline{\mathrm{N}}$ & $\begin{array}{c}\text { RA } \\
{[\mathrm{h} \mathrm{m} \mathrm{s}]}\end{array}$ & $\begin{array}{c}\text { Dec. } \\
{[\mathrm{d} \mathrm{m} \mathrm{s}] .}\end{array}$ & $\begin{array}{c}\delta_{r, d} \\
{["]} \\
\end{array}$ & $\begin{array}{c}\text { Offax } \\
{[\text { [] }}\end{array}$ & Net Cts. & $\begin{array}{c}\text { Exp. T. } \\
{[\mathrm{s}]}\end{array}$ & Pignif & $\overline{\bar{P} P_{K S}}$ & $\begin{array}{c}n_{H} \\
{\left[10^{22} \mathrm{~cm}^{-2}\right]} \\
\end{array}$ & $\overline{n_{H}(\mathrm{Ref})}$ & Mod & $\begin{array}{c}F_{X}[\mathrm{u}] \\
{\left[\mathrm{ergs} / \mathrm{s} / \mathrm{cm}^{2}\right]}\end{array}$ \\
\hline $\bar{~} 181$ & $6: 41: 01.1$ & $99: 34: 52.1$ & 0.22 & $\overline{0.9}$ & 147.1 & 249970 & $\overline{30.0}$ & 0.435 & 0.00 & $\bar{X}$ & $\overline{11 \mathrm{~T}}$ & -13.80 \\
\hline 182 & $6: 41: 01.2$ & $9: 36: 09.2$ & 0.29 & 2.0 & 67.0 & 92952 & 17.8 & 0.002 & 2.97 & $X$ & $1 \mathrm{~T}$ & -13.20 \\
\hline 183 & $6: 41: 01.3$ & $9: 34: 52.5$ & 0.26 & 0.9 & 80.9 & 36893 & 22.7 & 0.432 & 0.04 & $\mathrm{X}$ & $2 \mathrm{~T}$ & -13.70 \\
\hline 184 & 6:41:01.4 & 9:34:08.1 & 0.18 & 0.7 & 333.0 & 49310 & 44.8 & 0.556 & 0.04 & $\mathrm{Av}$ & $2 \mathrm{~T}$ & -13.35 \\
\hline 185 & $6: 41: 01.6$ & $9: 28: 13.2$ & 0.59 & 6.1 & 226.4 & 88904 & 33.2 & 0.070 & 0.08 & Av & $2 \mathrm{~T}$ & -13.70 \\
\hline 186 & $6: 41: 01.7$ & $9: 32: 60.0$ & 0.26 & 1.4 & 15.9 & 87754 & 6.8 & 0.555 & - & - & - & - \\
\hline 187 & $6: 41: 01.8$ & $9: 38: 40.7$ & 0.37 & 4.5 & 380.9 & 90472 & 43.5 & 0.248 & 0.00 & $\mathrm{X}$ & $2 \mathrm{Tab}$ & -13.62 \\
\hline 188 & $6: 41: 02.0$ & $9: 38: 32.9$ & 0.72 & 4.4 & 25.5 & 90706 & 10.8 & 0.199 & - & - & - & - \\
\hline 189 & $6: 41: 02.3$ & $9: 27: 23.7$ & 0.67 & 6.9 & 198.1 & 87370 & 26.1 & 0.002 & 0.19 & $\mathrm{X}$ & $1 \mathrm{~T}$ & -13.70 \\
\hline 190 & $6: 41: 02.5$ & $9: 34: 55.6$ & 0.16 & 1.2 & 614.0 & 82782 & 61.2 & 0.073 & 0.00 & $X$ & $1 \mathrm{~T}$ & -13.37 \\
\hline 191 & $6: 41: 02.6$ & $9: 36: 39.7$ & 0.38 & 2.6 & 19.2 & 47017 & 10.0 & 0.247 & 0.00 & JHK & - & -14.65 \\
\hline 192 & 6:41:02.6 & $9: 36: 15.3$ & 0.24 & 2.2 & 140.9 & 41221 & 25.3 & 0.110 & 2.17 & X & $1 \mathrm{~T}$ & -12.63 \\
\hline 193 & 6:41:02.6 & $9: 35: 12.9$ & 0.15 & 1.4 & 794.4 & 65563 & 69.2 & 0.000 & 0.06 & $\mathrm{Av}$ & $2 \mathrm{~T}$ & -13.02 \\
\hline 194 & $6: 41: 02.8$ & $9: 36: 16.0$ & 0.26 & 2.3 & 114.0 & 26949 & 22.5 & 0.034 & 2.57 & $X$ & $1 \mathrm{~T}$ & -12.72 \\
\hline 195 & $6: 41: 02.8$ & $9: 35: 34.5$ & 0.50 & 1.7 & 10.9 & 60549 & 5.7 & 0.016 & - & - & - & - \\
\hline 196 & $6: 41: 02.9$ & $9: 27: 23.4$ & 0.86 & 6.9 & 89.9 & 87361 & 15.6 & 0.352 & 0.00 & JHK & $1 \mathrm{~T}$ & -14.24 \\
\hline 197 & 6:41:03.0 & $9: 37: 36.0$ & 0.58 & 3.5 & 55.7 & 56589 & 16.9 & 0.377 & 0.49 & $\mathrm{Av}$ & $1 \mathrm{~T}$ & -13.89 \\
\hline 198 & 6:41:03.1 & $9: 30: 55.6$ & 0.74 & 3.5 & 5.1 & 93271 & 4.6 & 0.685 & - & - & - & - \\
\hline 199 & 6:41:03.1 & $9: 35: 44.7$ & 0.22 & 1.9 & 211.7 & 80125 & 32.5 & 0.338 & 3.17 & JHK & $1 \mathrm{~T}$ & -12.85 \\
\hline 200 & $6: 41: 03.2$ & $9: 26: 03.0$ & 1.02 & 8.3 & 126.5 & 79331 & 20.4 & 0.000 & 0.21 & $\mathrm{Av}$ & $1 \mathrm{~T}$ & -13.89 \\
\hline 201 & $6: 41: 03.2$ & $9: 32: 55.0$ & 0.38 & 1.7 & 19.8 & 92136 & 10.1 & 0.580 & 0.00 & JHK & - & -14.93 \\
\hline 202 & $6: 41: 03.4$ & $9: 36: 04.6$ & 0.28 & 2.2 & 87.2 & 72148 & 19.6 & 0.239 & 0.22 & $\mathrm{Av}$ & $1 \mathrm{~T}$ & -13.93 \\
\hline 203 & $6: 41: 03.4$ & $9: 40: 44.6$ & 1.32 & 6.6 & 116.1 & 86490 & 22.7 & 0.597 & 0.00 & Av & $1 \mathrm{~T}$ & -14.05 \\
\hline 204 & $6: 41: 03.4$ & 9:30:05.0 & 0.40 & 4.3 & 240.6 & 92050 & 37.8 & 0.000 & 0.00 & JHK & $1 \mathrm{~T}$ & -13.79 \\
\hline 205 & $6: 41: 03.4$ & $9: 32: 11.7$ & 0.28 & 2.4 & 64.9 & 92215 & 18.3 & 0.003 & 0.00 & JHK & $1 \mathrm{~T}$ & -14.24 \\
\hline 206 & $6: 41: 03.5$ & $9: 31: 18.4$ & 0.12 & 3.2 & 2022.9 & 93781 & 99.8 & 0.027 & 0.04 & Av & $2 \mathrm{~T}$ & -12.84 \\
\hline 207 & 6:41:03.6 & $9: 30: 29.0$ & 0.25 & 3.9 & 147.6 & 92621 & 23.5 & 0.941 & 0.23 & JHK & $1 \mathrm{~T}$ & -13.82 \\
\hline 208 & $6: 41: 03.6$ & $9: 36: 04.3$ & 0.32 & 2.2 & 55.2 & 88076 & 13.3 & 0.057 & 1.18 & $\mathrm{X}$ & $1 \mathrm{~T}$ & -13.56 \\
\hline 209 & $6: 41: 03.6$ & $9: 35: 43.4$ & 0.27 & 1.9 & 9.3 & 89314 & 6.2 & 0.677 & - & - & - & - \\
\hline 210 & $6: 41: 03.7$ & $9: 28: 20.1$ & 0.71 & 6.0 & 37.7 & 89118 & 11.0 & 0.985 & 0.20 & $\mathrm{Av}$ & - & -14.40 \\
\hline 211 & $6: 41: 03.7$ & $9: 27: 24.4$ & 1.49 & 6.9 & 39.1 & 87375 & 8.5 & 0.000 & - & - & - & - \\
\hline 212 & 6:41:03.7 & $9: 27: 39.8$ & 0.84 & 6.7 & 215.0 & 87862 & 32.2 & 0.113 & 0.24 & Av & $2 \mathrm{~T}$ & -13.45 \\
\hline 213 & 6:41:03.9 & $9: 37: 11.0$ & 0.42 & 3.2 & 54.0 & 50872 & 16.2 & 0.132 & 1.43 & JHK & - & -13.53 \\
\hline 214 & 6:41:04.0 & $9: 28: 24.5$ & 0.68 & 6.0 & 53.3 & 89246 & 12.0 & 0.055 & 0.00 & JHK & - & -14.48 \\
\hline 215 & $6: 41: 04.1$ & $9: 33: 48.6$ & 0.36 & 1.4 & 27.1 & 93012 & 11.4 & 0.411 & - & - & - & - \\
\hline 216 & $6: 41: 04.1$ & $9: 35: 21.0$ & 0.20 & 1.7 & 279.0 & 94048 & 40.7 & 0.516 & 0.00 & $\mathrm{Av}$ & $2 \mathrm{~T}$ & -13.73 \\
\hline 217 & $6: 41: 04.2$ & $9: 34: 57.1$ & 0.32 & 1.5 & 47.2 & 94252 & 15.7 & 0.147 & 0.00 & JHK & - & -14.56 \\
\hline 218 & $6: 41: 04.2$ & $9: 28: 40.6$ & 0.72 & 5.7 & 36.2 & 89741 & 10.6 & 0.004 & 0.00 & JHK & - & -14.65 \\
\hline 219 & 6:41:04.2 & $9: 36: 55.4$ & 0.41 & 3.0 & 19.5 & 86457 & 8.4 & 0.756 & 0.00 & JHK & - & -14.91 \\
\hline 220 & $6: 41: 04.3$ & $9: 35: 33.1$ & 0.51 & 1.9 & 8.0 & 93908 & 5.4 & 0.000 & 0.85 & JHK & - & -14.78 \\
\hline 221 & $6: 41: 04.3$ & $9: 35: 47.5$ & 0.31 & 2.1 & 46.1 & 93673 & 15.3 & 0.000 & 0.14 & $\mathrm{Av}$ & - & -14.40 \\
\hline 222 & $6: 41: 04.3$ & $9: 34: 56.1$ & 0.29 & 1.5 & 6.4 & 94256 & 4.8 & 0.947 & - & - & - & - \\
\hline 223 & $6: 41: 04.4$ & $9: 27: 30.1$ & 1.13 & 6.9 & 29.3 & 81902 & 7.6 & 0.029 & 0.00 & JHK & - & -14.71 \\
\hline 224 & $6: 41: 04.4$ & $9: 32: 57.3$ & 0.48 & 1.9 & 8.7 & 95281 & 6.3 & 0.992 & - & - & - & - \\
\hline 225 & 6:41:04.4 & $9: 36: 43.1$ & 0.46 & 2.9 & 19.3 & 87411 & 7.2 & 0.431 & 0.00 & JHK & - & -14.92 \\
\hline 226 & $6: 41: 04.5$ & 9:30:13.4 & 0.37 & 4.3 & 99.7 & 92221 & 21.9 & 0.331 & 0.00 & JHK & $1 \mathrm{~T}$ & -14.24 \\
\hline 227 & 6:41:04.6 & $9: 26: 09.5$ & 1.45 & 8.2 & 110.0 & 83905 & 18.9 & 0.019 & 0.04 & $\mathrm{Av}$ & $1 \mathrm{~T}$ & -14.05 \\
\hline 228 & $6: 41: 04.6$ & $9: 36: 18.0$ & 0.27 & 2.5 & 276.8 & 92907 & 40.1 & 0.000 & 6.18 & $X$ & $1 \mathrm{~T}$ & -12.72 \\
\hline 229 & 6:41:04.7 & $9: 36: 26.5$ & 0.18 & 2.7 & 505.5 & 92660 & 47.6 & 0.016 & 0.06 & $\mathrm{Av}$ & $2 \mathrm{~T}$ & -13.44 \\
\hline 230 & $6: 41: 05.0$ & $9: 42: 15.3$ & 1.22 & 8.2 & 16.1 & 83504 & 7.3 & 0.367 & - & - & - & - \\
\hline 231 & 6:41:05.1 & 9:33:00.2 & 0.16 & 2.0 & 200.1 & 95369 & 30.0 & 0.068 & 0.08 & Av & $1 \mathrm{~T}$ & -13.85 \\
\hline 232 & $6: 41: 05.2$ & $9: 36: 31.5$ & 0.51 & 2.8 & 11.9 & 92482 & 5.8 & 0.784 & - & - & - & - \\
\hline 233 & $6: 41: 05.2$ & 9:38:08.7 & 0.89 & 4.2 & 13.7 & 83965 & 6.6 & 0.496 & - & - & - & - \\
\hline 234 & 6:41:05.4 & $9: 33: 13.4$ & 0.11 & 1.9 & 2972.0 & 95370 & 136.8 & 0.000 & 0.06 & $\mathrm{X}$ & $2 \mathrm{Tab}$ & -12.60 \\
\hline 235 & 6:41:05.4 & $9: 36: 30.6$ & 0.26 & 2.8 & 118.6 & 92469 & 21.4 & 0.659 & 4.79 & X & $1 \mathrm{~T}$ & -13.00 \\
\hline 236 & $6: 41: 05.5$ & $9: 36: 26.4$ & 0.42 & 2.8 & 24.4 & 92575 & 8.0 & 0.026 & - & - & - & - \\
\hline 237 & $6: 41: 05.5$ & 9:34:08.0 & 0.49 & 1.7 & 9.3 & 40322 & 5.9 & 0.107 & - & - & - & - \\
\hline 238 & $6: 41: 05.5$ & $9: 31: 40.5$ & 0.21 & 3.1 & 228.5 & 94022 & 33.1 & 0.538 & 0.11 & $\mathrm{Av}$ & $1 \mathrm{~T}$ & -13.74 \\
\hline 239 & $6: 41: 05.6$ & $9: 35: 44.3$ & 0.35 & 2.3 & 36.1 & 93578 & 11.6 & 0.259 & - & - & - & - \\
\hline 240 & 6:41:05.7 & 9:31:01.3 & 0.18 & 3.7 & 565.1 & 93179 & 48.4 & 0.192 & 0.24 & $\mathrm{Av}$ & $2 \mathrm{~T}$ & -13.01 \\
\hline
\end{tabular}


Table 1. (Continued)

\begin{tabular}{|c|c|c|c|c|c|c|c|c|c|c|c|c|}
\hline$\overline{\mathrm{N}}$ & $\begin{array}{c}\text { RA } \\
{[\mathrm{h} \mathrm{m} \mathrm{s}]}\end{array}$ & $\begin{array}{c}\text { Dec. } \\
{[\mathrm{d} \mathrm{m} \mathrm{s}] .}\end{array}$ & $\begin{array}{l}\delta_{r, d} \\
{["]}\end{array}$ & $\begin{array}{c}\overline{\text { Offax }} \\
\text { ['] }\end{array}$ & Net Cts. & $\begin{array}{c}\text { Exp. T. } \\
{[\mathrm{s}]}\end{array}$ & $\overline{\overline{\text { Signif }}}$ & $\overline{\overline{P_{K S}}}$ & $\begin{array}{c}n_{H} \\
{\left[10^{22} \mathrm{~cm}^{-2}\right]} \\
\end{array}$ & $\overline{n_{H}(\mathrm{Ref})}$ & Mod & $\begin{array}{c}F_{X}[\mathrm{u}] \\
{\left[\mathrm{ergs} / \mathrm{s} / \mathrm{cm}^{2}\right]}\end{array}$ \\
\hline 241 & $6: 41: 05.8$ & $\overline{9: 36: 31.9}$ & 0.32 & $\overline{2.9}$ & $\overline{62.7}$ & $\overline{992395}$ & 14.8 & "0.001 & $\overline{0.33}$ & $\overline{\mathrm{X}}$ & $\overline{\overline{1 T}}$ & -14.12 \\
\hline 242 & $6: 41: 05.8$ & $9: 35: 29.3$ & 0.22 & 2.1 & 195.5 & 93851 & 29.8 & 0.000 & 3.98 & JHK & $1 \mathrm{~T}$ & -12.71 \\
\hline 243 & $6: 41: 05.8$ & $9: 31: 07.2$ & 0.57 & 3.6 & 22.9 & 93296 & 9.0 & 0.048 & - & - & - & - \\
\hline 244 & $6: 41: 05.9$ & $9: 34: 45.8$ & 0.15 & 1.8 & 807.5 & 94328 & 65.4 & 0.000 & 2.22 & X & $1 \mathrm{~T}$ & -12.57 \\
\hline 245 & $6: 41: 05.9$ & $9: 27: 17.7$ & 1.00 & 7.2 & 115.2 & 85076 & 20.8 & 0.004 & 0.09 & Av & $1 \mathrm{~T}$ & -14.03 \\
\hline 246 & $6: 41: 06.0$ & $9: 31: 39.8$ & 0.23 & 3.1 & 185.5 & 93948 & 29.0 & 0.000 & 0.17 & $\mathrm{X}$ & $1 \mathrm{~T}$ & -13.78 \\
\hline 247 & $6: 41: 06.0$ & $9: 35: 51.3$ & 0.54 & 2.4 & 17.1 & 93359 & 8.7 & 0.882 & 0.00 & JHK & - & -15.00 \\
\hline 248 & $6: 41: 06.1$ & $9: 34: 46.1$ & 0.20 & 1.9 & 131.0 & 94325 & 19.0 & 0.308 & 2.30 & $X$ & $1 \mathrm{~T}$ & -13.24 \\
\hline 249 & $6: 41: 06.2$ & $9: 36: 22.8$ & 0.17 & 2.8 & 2175.5 & 92576 & 117.6 & 0.000 & 0.08 & Av & $2 \mathrm{~T}$ & -12.72 \\
\hline 250 & $6: 41: 06.2$ & 9:33:08.8 & 0.25 & 2.1 & 100.4 & 95303 & 24.7 & 0.513 & 0.00 & Av & $1 \mathrm{~T}$ & -14.26 \\
\hline 251 & $6: 41: 06.2$ & $9: 29: 40.1$ & 0.74 & 4.9 & 27.0 & 87393 & 9.9 & 0.125 & 1.34 & JHK & - & -14.09 \\
\hline 252 & $6: 41: 06.3$ & $9: 29: 30.9$ & 0.52 & 5.1 & 107.4 & 87559 & 22.2 & 0.066 & 0.21 & Av & $1 \mathrm{~T}$ & -13.99 \\
\hline 253 & $6: 41: 06.3$ & $9: 37: 07.8$ & 0.43 & 3.4 & 21.3 & 91322 & 8.3 & 0.059 & - & - & - & - \\
\hline 254 & $6: 41: 06.4$ & $9: 28: 38.9$ & 0.59 & 5.9 & 78.5 & 89488 & 16.8 & 0.518 & 0.02 & $\mathrm{Av}$ & $1 \mathrm{~T}$ & -14.32 \\
\hline 255 & $6: 41: 06.4$ & $9: 26: 58.9$ & 0.57 & 7.5 & 1299.4 & 84665 & 75.7 & 0.000 & 0.95 & $X$ & $1 \mathrm{~T}$ & -12.51 \\
\hline 256 & $6: 41: 06.5$ & $9: 38: 37.2$ & 0.60 & 4.8 & 19.2 & 88794 & 7.8 & 0.000 & - & - & - & - \\
\hline 257 & $6: 41: 06.5$ & $9: 36: 05.2$ & 0.19 & 2.7 & 389.8 & 92966 & 39.8 & 0.000 & 3.82 & X & $1 \mathrm{~T}$ & -12.75 \\
\hline 258 & $6: 41: 06.6$ & $9: 35: 45.0$ & 0.41 & 2.5 & 19.0 & 93372 & 8.8 & 0.477 & 0.00 & Av & - & -14.95 \\
\hline 259 & $6: 41: 06.7$ & $9: 33: 54.2$ & 0.41 & 2.0 & 5.1 & 91547 & 4.7 & 0.877 & - & - & - & - \\
\hline 260 & $6: 41: 06.7$ & $9: 34: 45.8$ & 0.27 & 2.0 & 82.8 & 94300 & 20.2 & 0.316 & 0.81 & JHK & $1 \mathrm{~T}$ & -13.89 \\
\hline 261 & $6: 41: 06.8$ & $9: 27: 32.2$ & 0.53 & 7.0 & 1472.9 & 84815 & 87.4 & 0.000 & 0.00 & JHK & $2 \mathrm{~T}$ & -12.94 \\
\hline 262 & $6: 41: 06.9$ & $9: 40: 54.0$ & 1.85 & 7.0 & 13.8 & 47475 & 5.8 & 0.672 & 0.10 & JHK & - & -14.66 \\
\hline 263 & $6: 41: 06.9$ & $9: 29: 23.8$ & 0.58 & 5.2 & 73.4 & 89616 & 17.2 & 0.949 & 0.21 & Av & $1 \mathrm{~T}$ & -14.24 \\
\hline 264 & $6: 41: 07.1$ & $9: 30: 36.6$ & 0.47 & 4.2 & 46.2 & 88779 & 13.3 & 0.000 & - & - & - & - \\
\hline 265 & $6: 41: 07.2$ & $9: 27: 47.8$ & 0.50 & 6.8 & 534.2 & 86916 & 47.4 & 0.082 & 0.00 & $\mathrm{Av}$ & $1 \mathrm{~T}$ & -13.46 \\
\hline 266 & $6: 41: 07.2$ & $9: 42: 24.1$ & 2.25 & 8.4 & 19.1 & 38497 & 4.7 & 0.240 & 0.00 & JHK & - & -14.56 \\
\hline 267 & $6: 41: 07.3$ & $9: 36: 59.4$ & 0.31 & 3.5 & 1.6 & 91451 & 4.6 & 0.324 & - & - & - & - \\
\hline 268 & $6: 41: 07.3$ & $9: 31: 18.0$ & 0.36 & 3.6 & 98.1 & 87202 & 21.8 & 0.000 & 0.00 & JHK & $1 \mathrm{~T}$ & -14.28 \\
\hline 269 & $6: 41: 07.3$ & $9: 25: 55.1$ & 1.00 & 8.6 & 435.7 & 84465 & 43.2 & 0.383 & 0.24 & Av & $1 \mathrm{~T}$ & -13.34 \\
\hline 270 & $6: 41: 07.4$ & $9: 34: 54.7$ & 0.52 & 2.2 & 9.7 & 94096 & 5.3 & 0.619 & - & - & - & - \\
\hline 271 & $6: 41: 07.4$ & $9: 31: 10.9$ & 0.46 & 3.7 & 42.5 & 89025 & 13.1 & 0.097 & 0.07 & Av & - & -14.50 \\
\hline 272 & $6: 41: 07.4$ & $9: 34: 33.4$ & 0.40 & 2.2 & 5.7 & 94226 & 4.6 & 0.156 & 0.01 & Av & - & -15.48 \\
\hline 273 & $6: 41: 07.6$ & 9:30:00.6 & 0.53 & 4.8 & 91.2 & 91521 & 19.7 & 0.062 & 1.25 & JHK & $1 \mathrm{~T}$ & -13.60 \\
\hline 274 & $6: 41: 07.7$ & $9: 34: 19.1$ & 0.21 & 2.2 & 260.3 & 80631 & 34.4 & 0.000 & 8.92 & $\mathrm{X}$ & $1 \mathrm{~T}$ & -12.68 \\
\hline 275 & $6: 41: 07.7$ & $9: 41: 14.6$ & 0.91 & 7.3 & 67.4 & 78717 & 13.6 & 0.023 & 0.04 & Av & $1 \mathrm{~T}$ & -14.33 \\
\hline 276 & $6: 41: 07.8$ & $9: 28: 13.7$ & 0.54 & 6.4 & 350.0 & 88664 & 39.1 & 0.057 & 0.02 & Av & $1 \mathrm{~T}$ & -13.61 \\
\hline 277 & $6: 41: 08.0$ & $9: 34: 46.9$ & 0.53 & 2.3 & 21.7 & 93998 & 9.8 & 0.180 & 0.92 & JHK & - & -14.32 \\
\hline 278 & $6: 41: 08.0$ & $9: 30: 40.2$ & 0.30 & 4.2 & 229.4 & 92319 & 32.2 & 0.453 & 0.14 & $\mathrm{Av}$ & $2 \mathrm{~T}$ & -13.56 \\
\hline 279 & $6: 41: 08.2$ & 9:30:07.6 & 0.41 & 4.7 & 78.3 & 89617 & 17.2 & 0.028 & 2.66 & $X$ & $1 \mathrm{~T}$ & -13.43 \\
\hline 280 & $6: 41: 08.2$ & $9: 36: 55.9$ & 0.28 & 3.6 & 305.2 & 91378 & 38.5 & 0.000 & 1.33 & JHK & $1 \mathrm{~T}$ & -13.11 \\
\hline 281 & 6:41:08.2 & 9:34:09.6 & 0.49 & 2.3 & 5.9 & 44438 & 5.7 & 0.050 & 0.58 & JHK & - & -14.68 \\
\hline 282 & $6: 41: 08.2$ & $9: 38: 30.3$ & 0.49 & 4.9 & 430.5 & 88826 & 49.1 & 0.000 & 0.83 & JHK & $1 \mathrm{~T}$ & -13.07 \\
\hline 283 & $6: 41: 08.3$ & $9: 29: 39.6$ & 0.64 & 5.1 & 10.2 & 88980 & 6.5 & 0.734 & - & - & - & - \\
\hline 284 & $6: 41: 08.4$ & $9: 40: 35.7$ & 1.04 & 6.8 & 40.5 & 84970 & 9.8 & 0.546 & 0.00 & JHK & - & -14.58 \\
\hline 285 & $6: 41: 08.4$ & $9: 37: 52.4$ & 0.43 & 4.4 & 61.6 & 89842 & 16.0 & 0.133 & 0.00 & JHK & $1 \mathrm{~T}$ & -14.41 \\
\hline 286 & $6: 41: 08.5$ & $9: 31: 10.6$ & 0.49 & 3.9 & 8.7 & 92789 & 5.9 & 0.168 & - & - & - & - \\
\hline 287 & $6: 41: 08.5$ & 9:34:13.0 & 0.29 & 2.4 & 64.9 & 61899 & 17.4 & 0.528 & 2.79 & X & $1 \mathrm{~T}$ & -13.10 \\
\hline 288 & $6: 41: 08.6$ & 9:30:03.4 & 0.95 & 4.8 & 10.2 & 89454 & 5.8 & 0.409 & 0.00 & $\mathrm{Av}$ & - & -15.20 \\
\hline 289 & $6: 41: 08.7$ & $9: 38: 25.2$ & 0.90 & 4.8 & 10.6 & 88916 & 5.7 & 0.638 & - & - & - & - \\
\hline 290 & $6: 41: 08.9$ & $9: 41: 14.7$ & 1.00 & 7.4 & 425.2 & 83709 & 45.3 & 0.000 & 0.00 & Av & $1 \mathrm{~T}$ & -13.49 \\
\hline 291 & $6: 41: 09.0$ & $9: 33: 46.2$ & 0.58 & 2.6 & 13.0 & 93542 & 8.0 & 0.467 & 0.14 & Av & - & -14.94 \\
\hline 292 & $6: 41: 09.0$ & $9: 29: 07.5$ & 0.52 & 5.7 & 120.6 & 90074 & 21.1 & 0.000 & 0.80 & JHK & $1 \mathrm{~T}$ & -13.64 \\
\hline 293 & $6: 41: 09.1$ & 9:30:09.2 & 0.58 & 4.8 & 69.2 & 91479 & 17.0 & 0.032 & 0.21 & Av & $1 \mathrm{~T}$ & -14.15 \\
\hline 294 & $6: 41: 09.5$ & $9: 35: 25.3$ & 0.41 & 2.9 & 58.4 & 92982 & 16.8 & 0.790 & 0.00 & Av & $1 \mathrm{~T}$ & -14.52 \\
\hline 295 & $6: 41: 09.5$ & $9: 29: 36.7$ & 0.99 & 5.3 & 34.5 & 90759 & 9.8 & 0.014 & - & - & - & - \\
\hline 296 & $6: 41: 09.5$ & $9: 29: 25.2$ & 0.37 & 5.5 & 486.4 & 90464 & 42.8 & 0.000 & 1.92 & $\mathrm{X}$ & $1 \mathrm{~T}$ & -12.80 \\
\hline 297 & 6:41:09.6 & $9: 27: 57.6$ & 0.85 & 6.8 & 81.9 & 87174 & 15.3 & 0.060 & 0.00 & JHK & $1 \mathrm{~T}$ & -14.22 \\
\hline 298 & $6: 41: 09.6$ & $9: 36: 28.8$ & 0.51 & 3.5 & 28.0 & 91762 & 10.5 & 0.243 & 1.86 & JHK & - & -13.99 \\
\hline 299 & $6: 41: 09.8$ & $9: 29: 15.3$ & 0.62 & 5.7 & 20.4 & 90163 & 6.9 & 0.168 & - & - & - & - \\
\hline 300 & 6:41:09.8 & $9: 27: 12.2$ & 0.67 & 7.5 & 587.0 & 84781 & 50.7 & 0.746 & 0.19 & Av & $2 \mathrm{Tab}$ & -13.20 \\
\hline
\end{tabular}


Table 1. (Continued)

\begin{tabular}{|c|c|c|c|c|c|c|c|c|c|c|c|c|}
\hline $\mathrm{N}$ & $\begin{array}{c}\text { RA } \\
{[\mathrm{h} \mathrm{m} \mathrm{s}]}\end{array}$ & $\begin{array}{c}\text { Dec. } \\
{[\mathrm{d} \mathrm{m} \mathrm{s}] .}\end{array}$ & $\begin{array}{l}\delta_{r, d} \\
{["]}\end{array}$ & $\begin{array}{c}\text { Offax } \\
\text { ['] }\end{array}$ & Net Cts. & $\begin{array}{c}\text { Exp. T. } \\
{[\mathrm{s}]}\end{array}$ & Signif & $P_{K S}$ & $\begin{array}{c}n_{H} \\
{\left[10^{22} \mathrm{~cm}^{-2}\right]}\end{array}$ & $n_{H}($ Ref $)$ & Mod & $\begin{array}{c}F_{X}[\mathrm{u}] \\
{\left[\operatorname{ergs} / \mathrm{s} / \mathrm{cm}^{2}\right]}\end{array}$ \\
\hline 301 & 6:41:09.9 & 9:30:20.1 & 0.43 & $\overline{4.8}$ & $\overline{\overline{187.8}}$ & $\overline{91663}$ & $\overline{\overline{31.7}}$ & $\overline{\overline{0.530}}$ & $\overline{\overline{0.00}}$ & $\overline{\bar{X}}$ & $\overline{1 \mathrm{1T}}$ & -13.95 \\
\hline 302 & $6: 41: 10.0$ & $9: 27: 46.1$ & 0.45 & 7.0 & 2502.2 & 85776 & 120.7 & 0.000 & 0.00 & JHK & $2 \mathrm{~T}$ & -12.75 \\
\hline 303 & $6: 41: 10.0$ & $9: 29: 28.5$ & 0.80 & 5.5 & 24.5 & 90448 & 5.0 & 0.296 & - & - & - & - \\
\hline 304 & $6: 41: 10.1$ & $9: 31: 28.5$ & 0.35 & 3.9 & 41.5 & 92815 & 11.6 & 0.424 & 0.01 & $\mathrm{Av}$ & - & -14.61 \\
\hline 305 & $6: 41: 10.2$ & $9: 29: 34.2$ & 1.14 & 5.5 & 70.1 & 90572 & 15.7 & 0.000 & 4.84 & $\mathrm{X}$ & $1 \mathrm{~T}$ & -13.51 \\
\hline 306 & $6: 41: 10.2$ & $9: 30: 31.0$ & 0.76 & 4.7 & 22.6 & 91818 & 8.7 & 0.541 & - & - & - & - \\
\hline 307 & $6: 41: 10.3$ & $9: 33: 25.1$ & 0.31 & 3.0 & 58.6 & 93784 & 15.0 & 0.595 & 0.01 & Av & $1 \mathrm{~T}$ & -14.47 \\
\hline 308 & $6: 41: 10.3$ & $9: 34: 21.6$ & 0.39 & 2.8 & 25.7 & 89525 & 10.0 & 0.052 & - & - & - & - \\
\hline 309 & $6: 41: 10.3$ & $9: 28: 33.9$ & 0.87 & 6.3 & 21.6 & 85185 & 7.3 & 0.329 & 0.09 & $\mathrm{Av}$ & - & -14.74 \\
\hline 310 & $6: 41: 10.4$ & $9: 34: 18.6$ & 0.59 & 2.9 & 15.9 & 88925 & 7.9 & 0.163 & - & - & - & - \\
\hline 311 & $6: 41: 11.0$ & $9: 35: 55.5$ & 0.32 & 3.5 & 184.2 & 85551 & 29.9 & 0.000 & 0.35 & $\mathrm{Av}$ & $1 \mathrm{~T}$ & -13.61 \\
\hline 312 & $6: 41: 11.0$ & $9: 42: 08.3$ & 2.17 & 8.5 & 27.2 & 81862 & 6.1 & 0.000 & 0.19 & $\mathrm{Av}$ & - & -14.51 \\
\hline 313 & $6: 41: 11.0$ & $9: 34: 24.5$ & 0.31 & 3.0 & 2.9 & 92906 & 4.7 & 0.115 & - & - & - & - \\
\hline 314 & $6: 41: 11.4$ & $9: 29: 24.8$ & 0.71 & 5.7 & 6.9 & 88313 & 4.8 & 0.216 & - & - & - & - \\
\hline 315 & $6: 41: 11.6$ & $9: 29: 09.7$ & 1.26 & 6.0 & 18.1 & 86706 & 5.6 & 0.598 & - & - & - & - \\
\hline 316 & $6: 41: 11.8$ & $9: 31: 12.3$ & 0.55 & 4.4 & 19.1 & 92205 & 8.6 & 0.308 & 0.00 & JHK & - & -14.94 \\
\hline 317 & $6: 41: 11.8$ & $9: 35: 31.2$ & 0.72 & 3.5 & 26.5 & 92004 & 9.9 & 0.588 & - & - & - & - \\
\hline 318 & $6: 41: 11.8$ & $9: 26: 31.3$ & 0.82 & 8.4 & 306.7 & 84729 & 34.3 & 0.024 & 0.00 & $X$ & $1 \mathrm{~T}$ & -13.59 \\
\hline 319 & $6: 41: 11.9$ & $9: 37: 43.8$ & 0.86 & 4.8 & 18.8 & 89473 & 7.0 & 0.662 & 0.75 & JHK & - & -14.41 \\
\hline 320 & $6: 41: 12.2$ & $9: 29: 52.2$ & 0.62 & 5.5 & 49.2 & 88081 & 13.3 & 0.995 & - & - & - & - \\
\hline 321 & $6: 41: 12.2$ & $9: 29: 14.7$ & 1.20 & 6.0 & 21.0 & 89659 & 6.7 & 0.130 & - & - & - & - \\
\hline 322 & $6: 41: 12.3$ & $9: 40: 12.2$ & 1.02 & 6.8 & 16.1 & 85236 & 5.8 & 0.024 & 0.00 & JHK & - & -14.98 \\
\hline 323 & $6: 41: 12.5$ & $9: 35: 08.5$ & 0.72 & 3.5 & 23.8 & 92125 & 10.6 & 0.676 & 0.00 & JHK & - & -14.85 \\
\hline 324 & $6: 41: 12.6$ & $9: 29: 03.6$ & 0.66 & 6.2 & 119.3 & 89291 & 13.5 & 0.653 & 3.49 & $X$ & $1 \mathrm{~T}$ & -13.30 \\
\hline 325 & $6: 41: 12.9$ & $9: 35: 49.9$ & 0.73 & 3.8 & 23.1 & 91457 & 10.3 & 0.907 & 0.00 & JHK & - & -14.86 \\
\hline 326 & $6: 41: 13.0$ & $9: 35: 32.1$ & 0.29 & 3.7 & 83.3 & 91693 & 17.7 & 0.146 & 0.00 & $X$ & $1 \mathrm{~T}$ & -14.33 \\
\hline 327 & $6: 41: 13.0$ & $9: 29: 08.6$ & 0.68 & 6.2 & 489.0 & 89342 & 49.3 & 0.000 & 5.78 & $X$ & $1 \mathrm{~T}$ & -12.42 \\
\hline 328 & $6: 41: 13.0$ & $9: 27: 40.0$ & 0.63 & 7.5 & 347.7 & 81569 & 29.8 & 0.000 & 0.00 & $X$ & $2 \mathrm{~T}$ & -13.44 \\
\hline 329 & $6: 41: 13.0$ & $9: 27: 32.0$ & 0.48 & 7.6 & 867.2 & 79860 & 53.1 & 0.086 & 0.15 & $X$ & $1 \mathrm{~T}$ & -13.08 \\
\hline 330 & $6: 41: 13.1$ & $9: 31: 06.8$ & 0.66 & 4.7 & 39.4 & 89350 & 12.8 & 0.936 & 0.00 & $\mathrm{Av}$ & - & -14.61 \\
\hline 331 & $6: 41: 13.1$ & $9: 28: 58.8$ & 1.03 & 6.3 & 24.6 & 89057 & 5.2 & 0.222 & 0.06 & $\mathrm{Av}$ & - & -14.75 \\
\hline 332 & $6: 41: 13.2$ & $9: 26: 10.7$ & 0.79 & 8.8 & 1357.2 & 80837 & 76.9 & 0.000 & 0.05 & $X$ & $1 \mathrm{~T}$ & -12.90 \\
\hline 333 & $6: 41: 13.3$ & $9: 31: 50.4$ & 0.33 & 4.3 & 164.6 & 90527 & 26.6 & 0.666 & 0.04 & Av & $1 \mathrm{~T}$ & -13.94 \\
\hline 334 & $6: 41: 13.3$ & $9: 28: 07.5$ & 0.91 & 7.1 & 161.6 & 84565 & 26.0 & 0.285 & 0.00 & $\mathrm{X}$ & $1 \mathrm{~T}$ & -13.98 \\
\hline 335 & $6: 41: 13.4$ & $9: 38: 13.4$ & 0.78 & 5.4 & 25.5 & 84778 & 8.8 & 0.988 & 0.78 & JHK & - & -14.25 \\
\hline 336 & $6: 41: 13.8$ & $9: 40: 42.3$ & 2.37 & 7.5 & 17.3 & 84109 & 5.9 & 0.834 & - & - & - & - \\
\hline 337 & $6: 41: 13.8$ & $9: 28: 42.5$ & 0.91 & 6.7 & 15.9 & 79183 & 6.3 & 0.299 & - & - & - & - \\
\hline 338 & $6: 41: 14.1$ & $9: 26: 40.7$ & 1.09 & 8.5 & 336.1 & 83187 & 36.8 & 0.000 & 0.00 & $\mathrm{Av}$ & $2 \mathrm{~T}$ & -13.56 \\
\hline 339 & $6: 41: 14.4$ & $9: 26: 58.5$ & 1.53 & 8.2 & 41.1 & 83716 & 8.5 & 0.802 & 0.06 & $\mathrm{Av}$ & - & -14.50 \\
\hline 340 & $6: 41: 14.5$ & $9: 33: 21.3$ & 0.24 & 4.0 & 626.1 & 90446 & 53.4 & 0.000 & 0.00 & $X$ & $1 \mathrm{~T}$ & -13.38 \\
\hline 341 & $6: 41: 14.5$ & $9: 37: 14.2$ & 0.42 & 4.9 & 681.5 & 89492 & 67.0 & 0.000 & 0.00 & JHK & $2 \mathrm{~T}$ & -13.31 \\
\hline 342 & $6: 41: 14.7$ & $9: 34: 14.0$ & 0.92 & 3.9 & 11.6 & 91704 & 6.0 & 0.852 & 0.20 & $\mathrm{Av}$ & - & -14.92 \\
\hline 343 & $6: 41: 14.8$ & $9: 32: 35.9$ & 0.37 & 4.3 & 347.9 & 92232 & 44.5 & 0.089 & 0.09 & Av & $1 \mathrm{~T}$ & -13.54 \\
\hline 344 & $6: 41: 15.1$ & $9: 26: 44.4$ & 0.78 & 8.5 & 134.2 & 83131 & 17.8 & 0.094 & 0.12 & Av & $1 \mathrm{~T}$ & -13.80 \\
\hline 345 & $6: 41: 15.2$ & $9: 37: 57.6$ & 0.76 & 5.5 & 84.0 & 88340 & 18.7 & 0.064 & 0.02 & $\mathrm{Av}$ & $1 \mathrm{~T}$ & -14.28 \\
\hline 346 & $6: 41: 15.2$ & $9: 30: 16.5$ & 0.79 & 5.7 & 9.0 & 84924 & 4.7 & 0.050 & 0.00 & JHK & - & -15.23 \\
\hline 347 & $6: 41: 15.3$ & $9: 31: 16.5$ & 0.61 & 5.0 & 47.7 & 91408 & 14.9 & 0.230 & 0.00 & JHK & - & -14.54 \\
\hline 348 & $6: 41: 15.7$ & $9: 26: 33.7$ & 0.88 & 8.7 & 360.4 & 82717 & 30.6 & 0.729 & 0.11 & $X$ & $1 \mathrm{~T}$ & -13.50 \\
\hline 349 & $6: 41: 15.7$ & $9: 38: 18.1$ & 0.66 & 5.8 & 48.2 & 87696 & 12.3 & 0.842 & 0.00 & JHK & - & -14.52 \\
\hline 350 & $6: 41: 15.7$ & $9: 26: 17.1$ & 0.68 & 9.0 & 2334.2 & 84049 & 107.1 & 0.000 & 0.11 & Av & $2 \mathrm{~T}$ & -12.67 \\
\hline 351 & $6: 41: 15.9$ & $9: 30: 37.4$ & 0.73 & 5.6 & 14.0 & 84959 & 5.4 & 0.307 & 1.51 & JHK & - & -14.32 \\
\hline 352 & $6: 41: 16.7$ & $9: 29: 52.4$ & 0.54 & 6.2 & 356.9 & 87547 & 40.4 & 0.799 & 0.02 & $X$ & $1 \mathrm{~T}$ & -13.55 \\
\hline 353 & $6: 41: 16.8$ & $9: 27: 30.4$ & 1.17 & 8.1 & 226.2 & 85889 & 30.4 & 0.001 & 0.14 & Av & $2 \mathrm{~T}$ & -13.58 \\
\hline 354 & $6: 41: 16.8$ & $9: 36: 20.5$ & 0.42 & 4.9 & 250.3 & 89845 & 32.3 & 0.019 & 0.00 & Av & $1 \mathrm{~T}$ & -13.75 \\
\hline 355 & $6: 41: 17.0$ & $9: 32: 52.2$ & 0.47 & 4.7 & 50.9 & 90913 & 14.0 & 0.124 & 0.04 & $\mathrm{Av}$ & - & -14.48 \\
\hline 356 & $6: 41: 17.2$ & $9: 30: 12.1$ & 0.93 & 6.1 & 49.1 & 87777 & 12.3 & 0.418 & 1.62 & JHK & - & -13.77 \\
\hline 357 & $6: 41: 17.4$ & $9: 30: 29.0$ & 1.31 & 5.9 & 18.5 & 87760 & 5.9 & 0.120 & 1.48 & JHK & - & -14.22 \\
\hline 358 & $6: 41: 17.5$ & $9: 37: 15.3$ & 1.40 & 5.5 & 12.0 & 88627 & 4.6 & 0.289 & 0.00 & JHK & - & -15.13 \\
\hline 359 & $6: 41: 17.7$ & $9: 29: 26.3$ & 0.57 & 6.7 & 325.9 & 85711 & 36.5 & 0.749 & 0.00 & $\mathrm{Av}$ & $2 \mathrm{~T}$ & -13.64 \\
\hline 360 & $6: 41: 17.9$ & $9: 33: 36.9$ & 0.41 & 4.8 & 312.8 & 42406 & 36.6 & 0.000 & 0.00 & $\mathrm{Av}$ & $1 \mathrm{~T}$ & -13.16 \\
\hline
\end{tabular}


Table 1. (Continued)

\begin{tabular}{|c|c|c|c|c|c|c|c|c|c|c|c|c|}
\hline $\mathrm{N}$ & $\begin{array}{c}\text { RA } \\
{[\mathrm{h} \mathrm{m} \mathrm{s}]}\end{array}$ & $\begin{array}{c}\text { Dec. } \\
{[\mathrm{d} \mathrm{m} \mathrm{s}] .}\end{array}$ & $\begin{array}{l}\delta_{r, d} \\
{["]}\end{array}$ & $\begin{array}{c}\text { Offax } \\
{[']}\end{array}$ & Net Cts. & $\begin{array}{c}\text { Exp. T. } \\
{[\mathrm{s}]}\end{array}$ & $\overline{\overline{\text { Signif }}}$ & $P_{K S}$ & $\begin{array}{c}n_{H} \\
{\left[10^{22} \mathrm{~cm}^{-2}\right]}\end{array}$ & $\overline{\overline{n_{H}}(\text { Ref })}$ & $\overline{\text { Mod }}$ & $\begin{array}{c}F_{X}[\mathrm{u}] \\
{\left[\operatorname{ergs} / \mathrm{s} / \mathrm{cm}^{2}\right]}\end{array}$ \\
\hline$\overline{361}$ & $6: 41: 17.9$ & 9:29:01.1 & 1.17 & $\overline{\overline{7.0}}$ & $\overline{\overline{69.4}}$ & $\overline{87910}$ & $\overline{14.8}$ & $\overline{0.000}$ & $\overline{0.25}$ & $\overline{\overline{\mathrm{Av}}}$ & $\overline{1 \mathrm{1T}}$ & -14.18 \\
\hline 362 & $6: 41: 18.1$ & $9: 38: 25.2$ & 0.98 & 6.3 & 119.5 & 86898 & 22.7 & 0.000 & 0.00 & $\mathrm{X}$ & $1 \mathrm{~T}$ & -14.12 \\
\hline 363 & $6: 41: 18.2$ & $9: 28: 03.8$ & 1.95 & 7.8 & 43.9 & 86471 & 8.8 & 0.280 & - & - & - & - \\
\hline 364 & $6: 41: 18.3$ & $9: 33: 53.5$ & 0.41 & 4.8 & 285.7 & 86669 & 35.1 & 0.474 & 0.13 & $\mathrm{Av}$ & $1 \mathrm{~T}$ & -13.62 \\
\hline 365 & $6: 41: 18.3$ & $9: 28: 33.0$ & 1.37 & 7.5 & 110.7 & 87176 & 20.9 & 0.000 & 0.33 & $\mathrm{Av}$ & $2 \mathrm{~T}$ & -13.81 \\
\hline 366 & $6: 41: 18.3$ & $9: 29: 32.6$ & 1.12 & 6.7 & 88.5 & 88477 & 16.5 & 0.000 & 4.15 & $\mathrm{X}$ & $1 \mathrm{~T}$ & -13.19 \\
\hline 367 & $6: 41: 18.4$ & $9: 39: 41.1$ & 0.82 & 7.3 & 102.0 & 84931 & 17.0 & 0.603 & 0.00 & Av & $2 \mathrm{~T}$ & -14.09 \\
\hline 368 & $6: 41: 18.4$ & $9: 31: 29.8$ & 0.50 & 5.6 & 153.4 & 87437 & 23.0 & 0.020 & 0.00 & JHK & $1 \mathrm{~T}$ & -14.05 \\
\hline 369 & $6: 41: 18.8$ & $9: 30: 19.9$ & 1.30 & 6.3 & 8.3 & 89111 & 5.6 & 0.752 & 0.00 & JHK & - & -15.29 \\
\hline 370 & $6: 41: 18.9$ & $9: 27: 15.9$ & 0.83 & 8.6 & 119.0 & 82751 & 16.2 & 0.341 & 0.00 & Av & $2 \mathrm{~T}$ & -13.94 \\
\hline 371 & $6: 41: 19.0$ & $9: 35: 53.3$ & 1.73 & 5.3 & 13.1 & 89545 & 5.6 & 0.450 & 0.00 & JHK & - & -15.09 \\
\hline 372 & $6: 41: 19.2$ & $9: 30: 48.3$ & 1.24 & 6.1 & 14.7 & 89452 & 6.5 & 0.427 & 0.00 & $\mathrm{Av}$ & - & -15.04 \\
\hline 373 & $6: 41: 19.3$ & 9:38:06.9 & 1.87 & 6.4 & 13.7 & 85004 & 5.2 & 0.019 & 1.10 & JHK & - & -14.43 \\
\hline 374 & $6: 41: 19.4$ & $9: 30: 28.8$ & 2.44 & 6.3 & 15.9 & 89100 & 5.6 & 0.791 & 0.00 & $\mathrm{Av}$ & - & -15.01 \\
\hline 375 & $6: 41: 19.6$ & $9: 31: 44.4$ & 0.40 & 5.7 & 1168.4 & 86128 & 77.8 & 0.000 & 0.00 & $X$ & $1 \mathrm{~T}$ & -13.10 \\
\hline 376 & $6: 41: 19.7$ & $9: 31: 38.1$ & 0.99 & 5.8 & 37.6 & 89848 & 6.2 & 0.000 & - & - & - & _- \\
\hline 377 & $6: 41: 19.9$ & $9: 27: 05.1$ & 2.23 & 8.9 & 31.2 & 81754 & 6.6 & 0.438 & 0.00 & $\mathrm{Av}$ & - & -14.68 \\
\hline 378 & $6: 41: 20.0$ & $9: 32: 07.3$ & 1.38 & 5.7 & 9.5 & 87004 & 4.7 & 0.220 & - & - & - & - \\
\hline 379 & $6: 41: 20.6$ & $9: 38: 10.2$ & 1.78 & 6.7 & 5.2 & 86538 & 4.9 & 0.544 & - & - & - & - \\
\hline 380 & $6: 41: 20.7$ & $9: 32: 00.6$ & 1.02 & 5.9 & 31.1 & 89711 & 8.9 & 0.523 & - & - & - & - \\
\hline 381 & $6: 41: 20.7$ & $9: 30: 12.0$ & 0.85 & 6.8 & 215.2 & 88270 & 30.5 & 0.009 & 0.00 & JHK & $1 \mathrm{~T}$ & -13.85 \\
\hline 382 & $6: 41: 21.0$ & $9: 32: 41.2$ & 1.24 & 5.7 & 11.9 & 89835 & 6.2 & 0.206 & 0.00 & JHK & - & -15.14 \\
\hline 383 & $6: 41: 21.0$ & $9: 33: 36.3$ & 0.54 & 5.5 & 338.3 & 57602 & 40.1 & 0.040 & 0.09 & Av & $2 \mathrm{~T}$ & -13.34 \\
\hline 384 & $6: 41: 21.2$ & $9: 32: 14.7$ & 0.68 & 5.9 & 145.5 & 89645 & 24.4 & 0.135 & 0.00 & Av & $1 \mathrm{~T}$ & -14.07 \\
\hline 385 & $6: 41: 21.8$ & $9: 30: 39.9$ & 1.45 & 6.7 & 12.8 & 83562 & 4.8 & 0.632 & - & - & - & - \\
\hline 386 & $6: 41: 22.8$ & $9: 29: 39.2$ & 0.72 & 7.5 & 155.3 & 87156 & 21.5 & 0.078 & 0.00 & $\mathrm{Av}$ & $1 \mathrm{~T}$ & -13.97 \\
\hline 387 & $6: 41: 23.0$ & $9: 29: 30.6$ & 1.30 & 7.6 & 39.1 & 86383 & 6.7 & 0.000 & - & - & - & - \\
\hline 388 & $6: 41: 23.0$ & $9: 27: 26.9$ & 0.79 & 9.1 & 1324.1 & 84317 & 77.6 & 0.358 & 0.00 & JHK & $2 \mathrm{~T}$ & -13.02 \\
\hline 389 & $6: 41: 23.1$ & $9: 37: 33.6$ & 1.04 & 6.9 & 110.4 & 86004 & 20.0 & 0.000 & 0.18 & Av & $1 \mathrm{~T}$ & -13.84 \\
\hline 390 & $6: 41: 23.1$ & $9: 35: 22.0$ & 0.98 & 6.1 & 12.3 & 88078 & 5.9 & 0.100 & 0.00 & JHK & - & -15.11 \\
\hline 391 & $6: 41: 23.2$ & $9: 30: 36.5$ & 0.77 & 7.0 & 330.8 & 87716 & 39.2 & 0.000 & 0.00 & $\mathrm{X}$ & $1 \mathrm{~T}$ & -13.60 \\
\hline 392 & $6: 41: 23.4$ & $9: 38: 04.6$ & 1.90 & 7.2 & 31.2 & 84049 & 9.5 & 0.112 & 0.00 & JHK & - & -14.69 \\
\hline 393 & $6: 41: 23.5$ & $9: 41: 39.0$ & 3.87 & 9.6 & 42.2 & 79818 & 6.2 & 0.546 & - & - & - & - \\
\hline 394 & $6: 41: 23.8$ & $9: 33: 56.5$ & 0.77 & 6.2 & 30.9 & 88218 & 9.1 & 0.508 & 0.00 & JHK & - & -14.71 \\
\hline 395 & $6: 41: 24.2$ & $9: 31: 54.2$ & 0.74 & 6.7 & 362.6 & 88146 & 42.3 & 0.621 & 0.00 & JHK & $1 \mathrm{~T}$ & -13.62 \\
\hline 396 & $6: 41: 24.4$ & $9: 32: 45.5$ & 0.81 & 6.5 & 236.5 & 85706 & 33.9 & 0.137 & 0.00 & $\mathrm{Av}$ & $1 \mathrm{~T}$ & -13.79 \\
\hline 397 & $6: 41: 24.5$ & $9: 37: 35.5$ & 1.33 & 7.2 & 118.6 & 85785 & 22.2 & 0.056 & 0.00 & Av & $1 \mathrm{~T}$ & -14.13 \\
\hline 398 & $6: 41: 24.9$ & $9: 26: 21.9$ & 2.44 & 10.2 & 21.6 & 82280 & 5.5 & 0.868 & 0.00 & JHK & - & -14.84 \\
\hline 399 & $6: 41: 25.0$ & $9: 36: 00.1$ & 1.34 & 6.7 & 33.8 & 87013 & 10.6 & 0.324 & 0.00 & JHK & - & -14.67 \\
\hline 400 & $6: 41: 25.6$ & $9: 34: 42.8$ & 0.81 & 6.6 & 85.0 & 87293 & 16.9 & 0.457 & 0.02 & $\mathrm{Av}$ & $1 \mathrm{~T}$ & -14.31 \\
\hline 401 & $6: 41: 25.8$ & $9: 40: 36.4$ & 1.55 & 9.2 & 26.2 & 81607 & 7.0 & 0.731 & - & - & - & - \\
\hline 402 & $6: 41: 27.0$ & $9: 38: 41.2$ & 2.54 & 8.3 & 18.6 & 83756 & 5.2 & 0.171 & 0.00 & JHK & - & -14.91 \\
\hline 403 & $6: 41: 27.0$ & $9: 30: 13.1$ & 1.23 & 8.0 & 51.6 & 86173 & 13.4 & 0.073 & 0.15 & $\mathrm{Av}$ & $1 \mathrm{~T}$ & -14.25 \\
\hline 404 & $6: 41: 27.1$ & $9: 27: 03.6$ & 1.48 & 10.0 & 45.0 & 82702 & 9.3 & 0.727 & - & - & - & - \\
\hline 405 & $6: 41: 27.1$ & $9: 35: 06.1$ & 0.62 & 7.1 & 800.5 & 86524 & 60.2 & 0.001 & 0.08 & $\mathrm{Av}$ & $1 \mathrm{~T}$ & -13.20 \\
\hline 406 & $6: 41: 27.2$ & $9: 32: 06.7$ & 1.42 & 7.3 & 28.5 & 87113 & 8.8 & 0.039 & 0.00 & JHK & - & -14.74 \\
\hline 407 & $6: 41: 28.1$ & $9: 32: 50.4$ & 1.46 & 7.4 & 91.4 & 84426 & 16.7 & 0.000 & 0.00 & JHK & $1 \mathrm{~T}$ & -14.26 \\
\hline 408 & $6: 41: 28.7$ & $9: 38: 39.0$ & 1.05 & 8.6 & 420.3 & 83220 & 41.8 & 0.001 & 0.26 & JHK & $2 \mathrm{~T}$ & -13.29 \\
\hline 409 & $6: 41: 28.8$ & $9: 27: 10.7$ & 1.41 & 10.2 & 150.0 & 82389 & 18.3 & 0.562 & 0.08 & JHK & $1 \mathrm{~T}$ & -13.96 \\
\hline 410 & $6: 41: 28.8$ & $9: 34: 53.5$ & 2.23 & 7.5 & 18.1 & 83812 & 6.2 & 0.684 & 0.14 & JHK & - & -14.75 \\
\hline 411 & $6: 41: 29.1$ & $9: 39: 50.3$ & 1.36 & 9.3 & 111.4 & 81697 & 11.9 & 0.449 & 0.73 & X & $1 \mathrm{~T}$ & -13.62 \\
\hline 412 & $6: 41: 29.2$ & $9: 39: 35.7$ & 0.92 & 9.2 & 843.4 & 81946 & 56.8 & 0.029 & 0.11 & Av & $1 \mathrm{~T}$ & -13.15 \\
\hline 413 & $6: 41: 29.4$ & $9: 39: 18.0$ & 1.16 & 9.1 & 119.7 & 82211 & 17.1 & 0.060 & 0.09 & $\mathrm{Av}$ & $1 \mathrm{~T}$ & -14.03 \\
\hline 414 & $6: 41: 30.8$ & $9: 39: 00.6$ & 1.54 & 9.2 & 125.5 & 77599 & 16.2 & 0.092 & 1.49 & $X$ & $1 \mathrm{~T}$ & -13.40 \\
\hline 415 & $6: 41: 31.0$ & $9: 35: 25.3$ & 1.38 & 8.0 & 43.6 & 81908 & 9.9 & 0.030 & - & - & - & - \\
\hline 416 & $6: 41: 31.7$ & $9: 31: 54.1$ & 2.54 & 8.5 & 53.2 & 85099 & 9.3 & 0.994 & 0.00 & $\mathrm{Av}$ & - & -14.46 \\
\hline 417 & $6: 41: 32.5$ & $9: 38: 07.2$ & 1.00 & 9.2 & 564.1 & 82268 & 45.0 & 0.022 & 0.03 & $\mathrm{Av}$ & $1 \mathrm{~T}$ & -13.39 \\
\hline 418 & $6: 41: 34.5$ & $9: 36: 32.2$ & 1.61 & 9.1 & 80.2 & 78097 & 13.4 & 0.017 & 0.02 & $\mathrm{Av}$ & $1 \mathrm{~T}$ & -14.30 \\
\hline 419 & $6: 41: 36.2$ & $9: 39: 20.6$ & 2.12 & 10.5 & 104.6 & 79023 & 15.4 & 0.847 & 0.04 & $\mathrm{Av}$ & $1 \mathrm{~T}$ & -14.15 \\
\hline 420 & $6: 41: 39.6$ & $9: 40: 28.7$ & 2.24 & 11.8 & 308.3 & 76522 & 25.2 & 0.001 & 0.11 & Av & $1 \mathrm{~T}$ & -13.51 \\
\hline
\end{tabular}


Table 3. Master catalogs of objects in the ACIS FOV.

\begin{tabular}{|c|c|c|c|c|c|c|c|c|c|c|c|}
\hline$\overline{\mathrm{N}}$ & $\begin{array}{c}\text { RA } \\
{[\mathrm{h} \mathrm{m} \mathrm{s}]}\end{array}$ & $\begin{array}{c}\text { Dec. } \\
{[\mathrm{d} \mathrm{m} \mathrm{s}]}\end{array}$ & $\begin{array}{c}\text { Id. rad. } \\
\text { ["] }\end{array}$ & ACIS & 2Mass & Reb.+ & $\overline{\text { Lamm+ }}$ & Flacc+ & 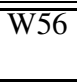 & $\overline{\overline{\text { Dahm+ }}}$ & $\begin{array}{l}\text { Ct. Rate. } \\
{\left[10^{-4} s^{-1}\right]}\end{array}$ \\
\hline$\overline{11}$ & $6: 40: 22.4$ & $\overline{99: 28: 03.2}$ & $\overline{0.54}$ & $\overline{-1}$ & $06402243+0928032$ & $\overline{-1}$ & $\overline{\overline{3228}}$ & $\overline{-1}$ & $\overline{-1}$ & $\overline{-1}$ & $\overline{<<8.7}$ \\
\hline 2 & $6: 40: 24.2$ & $9: 30: 38.1$ & 0.50 & - & $06402419+0930381$ & 2229 & - & - & - & - & $<3.3$ \\
\hline 3 & $6: 40: 24.3$ & $9: 27: 44.4$ & 0.50 & - & $06402425+0927443$ & - & - & - & - & - & $<23.5$ \\
\hline 4 & $6: 40: 24.8$ & $9: 29: 39.6$ & 0.66 & - & $06402475+0929395$ & - & - & - & - & - & $<3.4$ \\
\hline 5 & $6: 40: 24.8$ & $9: 28: 42.6$ & 0.50 & - & $06402482+0928425$ & - & - & - & - & - & $<3.3$ \\
\hline 6 & $6: 40: 24.8$ & $9: 28: 59.8$ & 0.64 & - & $06402482+0928597$ & - & - & - & - & - & $<3.5$ \\
\hline 7 & $6: 40: 25.0$ & $9: 30: 00.5$ & 1.00 & - & - & - & 3295 & - & - & - & $<3.0$ \\
\hline $8 *$ & $6: 40: 25.0$ & $9: 32: 08.4$ & 0.50 & - & $06402504+0932084$ & 2258 & 3299 & - & - & - & $<4.1$ \\
\hline 9 & $6: 40: 25.1$ & $9: 29: 36.9$ & 0.50 & - & $06402505+0929369$ & 2261 & 3300 & - & - & - & $<3.4$ \\
\hline 10 & $6: 40: 25.4$ & $9: 30: 26.4$ & 1.00 & - & - & - & 3326 & - & - & - & $<2.8$ \\
\hline 11 & $6: 40: 25.5$ & $9: 29: 44.6$ & 1.00 & - & - & - & 3331 & - & - & - & $<3.2$ \\
\hline 12 & $6: 40: 25.5$ & 9:29:54.4 & 1.00 & - & - & - & 3334 & - & - & - & $<2.9$ \\
\hline 13 & $6: 40: 25.6$ & 9:32:35.9 & 0.50 & - & $06402559+0932358$ & - & 3348 & - & - & - & $<2.9$ \\
\hline 14 & $6: 40: 25.7$ & 9:33:05.7 & 1.00 & - & - & - & 3351 & - & - & - & $<17.0$ \\
\hline 15 & $6: 40: 25.7$ & 9:29:09.8 & 1.00 & - & - & - & 3352 & - & - & - & $<3.4$ \\
\hline $16^{*}$ & $6: 40: 25.8$ & $9: 29: 23.3$ & 0.50 & 1 & $06402575+0929232$ & 2284 & 3356 & - & - & - & 3.5 \\
\hline 17 & $6: 40: 25.8$ & $9: 32: 36.4$ & 0.50 & - & $06402580+0932363$ & - & 3364 & - & - & - & $<2.7$ \\
\hline 18 & $6: 40: 25.8$ & $9: 30: 20.5$ & 1.00 & - & - & - & 3363 & - & - & - & $<2.7$ \\
\hline 19 & $6: 40: 25.9$ & 9:33:08.9 & 1.00 & - & - & - & 3371 & - & - & - & $<4.5$ \\
\hline 20 & $6: 40: 26.0$ & 9:30:23.1 & 0.57 & - & $06402604+0930231$ & 2295 & 3379 & - & - & - & $<2.7$ \\
\hline 21 & $6: 40: 26.2$ & $9: 30: 12.2$ & 1.00 & - & - & - & 3391 & - & - & - & $<2.8$ \\
\hline 22 & $6: 40: 26.2$ & 9:30:19.9 & 1.00 & - & - & - & 3393 & - & - & - & $<2.7$ \\
\hline 23 & $6: 40: 26.2$ & $9: 30: 47.8$ & 0.50 & - & $06402621+0930477$ & 2301 & 3395 & - & - & - & $<2.8$ \\
\hline 24 & $6: 40: 26.2$ & $9: 32: 36.3$ & 1.00 & - & - & - & 3396 & - & - & - & $<2.5$ \\
\hline 25 & $6: 40: 26.3$ & $9: 30: 30.2$ & 1.00 & - & - & - & 3398 & - & - & - & $<2.7$ \\
\hline 26 & $6: 40: 26.4$ & 9:28:44.1 & 0.50 & - & $06402635+0928440$ & 2305 & 3406 & - & - & - & $<3.1$ \\
\hline 27 & $6: 40: 26.4$ & 9:33:51.6 & 0.50 & - & $06402638+0933515$ & 2306 & 3409 & - & - & - & $<3.0$ \\
\hline 28 & $6: 40: 26.5$ & 9:31:48.9 & 0.50 & - & $06402648+0931488$ & 2313 & 3417 & - & - & - & $<2.7$ \\
\hline $29 *$ & $6: 40: 26.5$ & $9: 29: 22.1$ & 0.50 & - & $06402649+0929220$ & 2314 & 3421 & - & - & - & $<3.2$ \\
\hline 30 & $6: 40: 26.6$ & $9: 33: 25.0$ & 0.50 & - & $06402656+0933250$ & 2317 & 3427 & - & 49 & - & $<2.5$ \\
\hline 31 & $6: 40: 26.6$ & $9: 27: 58.0$ & 0.50 & - & $06402664+0927579$ & 2319 & 3433 & - & - & - & $<3.7$ \\
\hline 32 & $6: 40: 26.7$ & $9: 29: 59.8$ & 1.00 & - & - & - & 3434 & - & - & - & $<2.8$ \\
\hline 33 & $6: 40: 26.7$ & $9: 31: 38.6$ & 1.00 & - & - & - & 3435 & - & - & - & $<2.7$ \\
\hline 34 & $6: 40: 26.8$ & 9:33:42.3 & 0.50 & - & $06402684+0933422$ & - & 3449 & - & - & - & $<2.4$ \\
\hline 35 & $6: 40: 26.8$ & $9: 29: 48.9$ & 1.00 & - & - & - & 3448 & - & - & - & $<2.9$ \\
\hline 36 & $6: 40: 26.9$ & $9: 32: 56.0$ & 1.00 & - & - & - & 3451 & - & - & - & $<2.5$ \\
\hline 37 & $6: 40: 27.1$ & 9:32:05.0 & 1.00 & - & - & - & 3459 & - & - & - & $<4.6$ \\
\hline 38 & $6: 40: 27.1$ & $9: 31: 57.6$ & 1.00 & - & - & - & 3463 & - & - & - & $<7.5$ \\
\hline 39 & $6: 40: 27.1$ & $9: 32: 31.2$ & 0.50 & - & $06402710+0932311$ & 2326 & 3464 & - & - & - & $<2.4$ \\
\hline 40 & $6: 40: 27.1$ & $9: 29: 30.0$ & 0.50 & - & $06402710+0929299$ & 2330 & 3462 & - & - & - & $<3.1$ \\
\hline 41 & $6: 40: 27.1$ & 9:29:00.6 & 1.00 & - & - & - & 3466 & - & - & - & $<3.0$ \\
\hline 42 & $6: 40: 27.2$ & $9: 28: 43.0$ & 0.50 & - & $06402716+0928430$ & 2329 & 3471 & - & - & - & $<3.1$ \\
\hline 43 & $6: 40: 27.2$ & $9: 30: 56.2$ & 1.00 & - & - & - & 3474 & - & - & - & $<2.9$ \\
\hline 44 & $6: 40: 27.2$ & $9: 32: 26.5$ & 0.50 & - & $06402724+0932264$ & - & 3476 & - & - & - & $<2.4$ \\
\hline 45 & $6: 40: 27.3$ & $9: 29: 29.4$ & 1.00 & - & - & - & 3478 & - & - & - & $<3.1$ \\
\hline 46 & $6: 40: 27.4$ & $9: 33: 54.9$ & 14.50 & - & - & - & - & 11 & - & - & $<2.4$ \\
\hline $47 *$ & $6: 40: 27.7$ & 9:31:59.7 & 0.50 & 2 & $06402772+0931596$ & 2344 & 3518 & - & - & 37 & 10.0 \\
\hline 48 & $6: 40: 27.8$ & 9:30:01.8 & 1.00 & - & - & - & 3527 & - & - & - & $<2.8$ \\
\hline 49 & $6: 40: 28.0$ & 9:32:34.1 & 1.00 & - & - & - & 3531 & - & - & - & $<2.3$ \\
\hline 50 & $6: 40: 28.0$ & $9: 32: 19.2$ & 1.00 & - & - & - & 3535 & - & - & - & $<2.4$ \\
\hline 51 & $6: 40: 28.0$ & $9: 34: 57.6$ & 0.50 & - & $06402802+0934575$ & - & 3539 & - & - & - & $<2.4$ \\
\hline 52 & $6: 40: 28.1$ & $9: 31: 36.1$ & 0.57 & - & $06402805+0931360$ & 2350 & 3543 & - & - & - & $<2.5$ \\
\hline $53 *$ & $6: 40: 28.1$ & $9: 35: 33.8$ & 0.50 & 3 & $06402807+0935337$ & 2351 & 3546 & - & - & - & 5.2 \\
\hline 54 & $6: 40: 28.1$ & $9: 30: 58.4$ & 0.50 & - & $06402810+0930584$ & - & 3549 & $13 \dagger$ & - & - & $<2.9$ \\
\hline 55 & $6: 40: 28.2$ & $9: 32: 07.8$ & 1.00 & - & - & - & 3556 & - & - & - & $<3.8$ \\
\hline 56 & $6: 40: 28.2$ & $9: 35: 12.0$ & 0.50 & - & $06402817+0935120$ & - & 3558 & - & - & - & $<2.6$ \\
\hline 57 & $6: 40: 28.3$ & $9: 31: 11.2$ & 0.75 & - & $06402832+0931112$ & - & 3570 & $13 \dagger$ & - & - & $<2.7$ \\
\hline 58 & $6: 40: 28.4$ & $9: 30: 24.8$ & 1.00 & - & - & - & 3579 & - & - & - & $<2.7$ \\
\hline 59 & $6: 40: 28.5$ & $9: 28: 31.3$ & 0.69 & - & $06402846+0928313$ & - & 3580 & - & - & - & $<3.0$ \\
\hline 60 & $6: 40: 28.5$ & $9: 32: 55.1$ & 0.51 & - & $06402847+0932551$ & 2365 & 3581 & - & - & - & $<2.4$ \\
\hline
\end{tabular}


Table 3. (Continued)

\begin{tabular}{|c|c|c|c|c|c|c|c|c|c|c|c|}
\hline $\mathrm{N}$ & $\begin{array}{c}\mathrm{RA} \\
{[\mathrm{h} \mathrm{m} \mathrm{s}]}\end{array}$ & $\begin{array}{c}\text { Dec. } \\
{[\mathrm{d} \mathrm{m} \mathrm{s}]}\end{array}$ & $\begin{array}{c}\text { Id. rad. } \\
{["]} \\
\end{array}$ & ACIS & 2Mass & Reb.+ & $\overline{\text { Lamm+ }}$ & Flacc+ & $\overline{\text { W56 }}$ & $\overline{\text { Dahm+ }}$ & $\begin{array}{l}\text { Ct. Rate. } \\
{\left[10^{-4} s^{-1}\right]} \\
\end{array}$ \\
\hline$\overline{61}$ & $6: 40: 28.5$ & 9 9:33:47.1 & $\overline{0.50}$ & 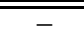 & $06402850+0933470$ & 2368 & 3584 & - & - & - & $\overline{<<2.2}$ \\
\hline 62 & $6: 40: 28.6$ & 9:36:08.2 & 1.00 & - & - & - & 3587 & - & - & - & $<3.3$ \\
\hline 63 & $6: 40: 28.6$ & $9: 30: 26.0$ & 0.50 & - & $06402857+0930260$ & 2372 & 3590 & - & - & - & $<2.6$ \\
\hline 64 & $6: 40: 28.6$ & $9: 30: 57.3$ & 1.00 & - & - & - & 3591 & - & - & - & $<121.3$ \\
\hline $65^{*}$ & $6: 40: 28.6$ & $9: 35: 47.6$ & 0.50 & 4 & $06402858+0935476$ & 2373 & 3592 & 12 & 51 & - & 29.1 \\
\hline 66 & $6: 40: 28.7$ & 9:34:49.9 & 0.50 & - & $06402866+0934498$ & - & 3599 & - & - & - & $<2.3$ \\
\hline 67 & $6: 40: 28.7$ & $9: 27: 36.6$ & 1.00 & - & - & - & 3600 & - & - & - & $<4.5$ \\
\hline $68 *$ & $6: 40: 28.8$ & 9:31:00.3 & 0.50 & 5 & $06402877+0931002$ & 2378 & 3603 & $13 \dagger$ & - & 40 & 153.6 \\
\hline 69 & $6: 40: 28.8$ & 9:33:16.0 & 1.00 & - & - & - & 3606 & - & - & - & $<2.3$ \\
\hline $70 *$ & $6: 40: 28.8$ & $9: 31: 01.8$ & 1.00 & - & - & - & 3609 & - & - & 41 & $<121.3$ \\
\hline $71 *$ & $6: 40: 28.9$ & 9:33:05.6 & 0.50 & 6 & $06402886+0933055$ & 2382 & 3612 & - & - & - & 14.2 \\
\hline 72 & $6: 40: 29.0$ & $9: 28: 33.9$ & 1.00 & - & - & - & 3620 & - & - & - & $<2.8$ \\
\hline 73 & 6:40:29.1 & $9: 29: 38.4$ & 1.00 & - & - & - & 3622 & - & - & - & $<2.7$ \\
\hline 74 & $6: 40: 29.2$ & $9: 29: 29.6$ & 0.84 & - & $06402919+0929296$ & 2386 & 3626 & - & - & - & $<2.7$ \\
\hline 75 & $6: 40: 29.2$ & $9: 29: 39.2$ & 1.00 & - & - & - & 3632 & - & - & - & $<2.7$ \\
\hline 76 & $6: 40: 29.7$ & $9: 37: 45.3$ & 1.00 & - & - & - & 3664 & - & - & - & $<2.7$ \\
\hline 77 & $6: 40: 29.8$ & $9: 31: 58.4$ & 1.00 & - & - & - & 3665 & - & - & - & $<2.3$ \\
\hline 78 & $6: 40: 29.8$ & $9: 28: 03.7$ & 0.50 & - & $06402977+0928036$ & 2413 & 3668 & - & - & - & $<3.0$ \\
\hline 79 & $6: 40: 29.8$ & 9:30:55.9 & 1.00 & - & - & - & 3672 & - & - & - & $<2.9$ \\
\hline 80 & 6:40:29.9 & 9:29:15.2 & 1.00 & - & - & - & 3679 & - & - & - & $<2.6$ \\
\hline 81 & $6: 40: 29.9$ & 9:28:29.9 & 1.00 & - & - & - & 3682 & - & - & - & $<2.8$ \\
\hline 82 & $6: 40: 29.9$ & $9: 32: 21.9$ & 1.00 & - & - & - & 3684 & - & - & - & $<2.2$ \\
\hline 83 & 6:40:29.9 & 9:33:43.0 & 1.00 & - & - & - & 3686 & - & - & - & $<2.0$ \\
\hline 84 & $6: 40: 30.0$ & 9:38:07.6 & 1.00 & - & - & - & 3691 & - & - & - & $<2.8$ \\
\hline 85 & $6: 40: 30.0$ & $9: 34: 43.3$ & 0.50 & - & $06403004+0934433$ & 2424 & 3695 & - & - & - & $<2.2$ \\
\hline 86 & $6: 40: 30.2$ & 9:30:48.3 & 0.50 & - & $06403015+0930483$ & 2428 & 3699 & - & - & - & $<2.7$ \\
\hline 87 & $6: 40: 30.2$ & 9:28:08.4 & 0.50 & - & $06403017+0928084$ & 2429 & 3702 & - & - & - & $<2.9$ \\
\hline 88 & $6: 40: 30.2$ & $9: 31: 48.3$ & 1.00 & - & - & - & 3708 & - & - & - & $<2.2$ \\
\hline 89 & $6: 40: 30.2$ & $9: 31: 51.7$ & 1.00 & - & - & - & 3709 & - & - & - & $<2.2$ \\
\hline 90 & $6: 40: 30.3$ & $9: 34: 42.6$ & 1.00 & - & - & - & 3713 & - & - & - & $<2.2$ \\
\hline 91 & $6: 40: 30.3$ & 9:30:04.4 & 0.50 & - & $06403028+0930044$ & 2433 & 3715 & - & - & - & $<2.5$ \\
\hline 92 & $6: 40: 30.3$ & $9: 30: 57.0$ & 1.00 & - & - & - & 3717 & - & - & - & $<2.7$ \\
\hline 93 & $6: 40: 30.5$ & $9: 30: 20.4$ & 0.50 & - & $06403051+0930203$ & 2440 & 3729 & - & - & - & $<2.4$ \\
\hline 94 & $6: 40: 30.6$ & 9:38:39.6 & 1.00 & 7 & - & - & 3733 & - & - & - & 2.2 \\
\hline 95 & $6: 40: 30.6$ & $9: 30: 59.4$ & 1.00 & - & - & - & 3735 & - & - & - & $<2.5$ \\
\hline 96 & $6: 40: 30.8$ & $9: 36: 40.8$ & 0.76 & - & $06403077+0936408$ & - & 3742 & - & - & - & $<2.1$ \\
\hline 97 & $6: 40: 30.8$ & $9: 35: 37.1$ & 0.50 & - & $06403081+0935370$ & - & 3747 & - & - & - & $<2.2$ \\
\hline $98^{*}$ & $6: 40: 30.9$ & $9: 34: 40.6$ & 0.50 & 8 & $06403086+0934405$ & 2456 & 3748 & - & 55 & 49 & 19.0 \\
\hline 99 & $6: 40: 30.9$ & $9: 36: 16.5$ & 0.50 & - & $06403088+0936165$ & - & 3755 & - & - & - & $<2.2$ \\
\hline 100 & $6: 40: 30.9$ & $9: 30: 29.0$ & 1.00 & - & - & - & 3754 & - & - & - & $<2.3$ \\
\hline 101 & $6: 40: 31.0$ & $9: 33: 59.5$ & 0.50 & - & 06403104+0933594 & 2461 & 3762 & - & - & - & $<1.9$ \\
\hline 102 & $6: 40: 31.1$ & $9: 35: 40.2$ & 0.50 & - & $06403106+0935401$ & 2462 & 3763 & - & - & - & $<2.6$ \\
\hline 103 & $6: 40: 31.1$ & $9: 34: 46.6$ & 0.50 & - & $06403108+0934465$ & - & - & - & - & - & $<12.3$ \\
\hline 104 & $6: 40: 31.1$ & $9: 30: 16.1$ & 1.00 & - & - & - & 3765 & - & - & - & $<2.4$ \\
\hline $105^{*}$ & $6: 40: 31.1$ & 9:31:08.1 & 1.00 & - & - & - & 3766 & - & - & - & $<43.7$ \\
\hline 106 & $6: 40: 31.2$ & $9: 27: 26.3$ & 1.00 & - & - & - & 3771 & - & - & - & $<6.1$ \\
\hline 107 & $6: 40: 31.2$ & 9:31:02.6 & 1.00 & - & - & - & 3773 & - & - & - & $<43.7$ \\
\hline $108 *$ & $6: 40: 31.2$ & 9:31:07.1 & 0.50 & 9 & $06403123+0931071$ & 2465 & 3778 & 16 & 56 & 51 & 58.1 \\
\hline 109 & $6: 40: 31.3$ & 9:29:43.0 & 0.52 & - & $06403128+0929430$ & - & 3779 & - & - & - & $<2.5$ \\
\hline 110 & $6: 40: 31.3$ & 9:39:12.4 & 1.00 & - & - & - & 3781 & - & - & - & $<2.7$ \\
\hline 111 & $6: 40: 31.3$ & 9:35:29.6 & 0.50 & - & $06403129+0935296$ & 2468 & 3780 & - & - & - & $<2.0$ \\
\hline 112 & $6: 40: 31.3$ & $9: 33: 44.1$ & 1.00 & - & - & - & 3783 & - & - & - & $<1.9$ \\
\hline 113 & $6: 40: 31.4$ & $9: 32: 52.6$ & 0.50 & - & $06403135+0932525$ & 2470 & 3786 & - & - & - & $<1.9$ \\
\hline 114 & $6: 40: 31.4$ & 9:32:59.4 & 0.50 & - & $06403135+0932594$ & - & 3787 & - & - & - & $<1.9$ \\
\hline 115 & $6: 40: 31.4$ & 9:30:10.9 & 0.50 & - & $06403142+0930109$ & - & 3790 & - & - & - & $<2.4$ \\
\hline 116 & $6: 40: 31.5$ & $9: 27: 55.4$ & 1.00 & - & - & - & 3796 & - & - & - & $<2.7$ \\
\hline 117 & $6: 40: 31.6$ & $9: 40: 22.4$ & 1.00 & - & - & - & 3801 & - & - & - & $<17.5$ \\
\hline $118^{*}$ & $6: 40: 31.6$ & $9: 36: 15.8$ & 0.50 & - & $06403158+0936157$ & 2472 & 3803 & - & - & - & $<2.1$ \\
\hline 119 & $6: 40: 31.6$ & $9: 31: 41.9$ & 1.00 & - & - & - & 3804 & - & - & - & $<2.0$ \\
\hline 120 & $6: 40: 31.6$ & $9: 40: 21.9$ & 1.00 & - & - & - & 3806 & - & - & - & $<13.7$ \\
\hline
\end{tabular}


Table 3. (Continued)

\begin{tabular}{|c|c|c|c|c|c|c|c|c|c|c|c|}
\hline$\overline{\mathrm{N}}$ & $\begin{array}{c}\mathrm{RA} \\
{[\mathrm{h} \mathrm{m} \mathrm{s}]}\end{array}$ & $\begin{array}{c}\text { Dec. } \\
{[\mathrm{d} \mathrm{m} \mathrm{s}]}\end{array}$ & $\begin{array}{c}\text { Id. rad. } \\
\text { ["] } \\
\end{array}$ & ACIS & 2Mass & Reb.+ & $\overline{\text { Lamm+ }}$ & Flacc+ & $\overline{\text { W56 }}$ & $\overline{\overline{\text { Dahm+ }}}$ & $\begin{array}{l}\text { Ct. Rate. } \\
{\left[10^{-4} s^{-1}\right]}\end{array}$ \\
\hline$\overline{121}$ & $6: 40: 31.7$ & $9: 27: 30.7$ & 1.00 & $\overline{-}$ & $\overline{-1}$ & $\overline{-1}$ & 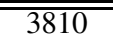 & - & - & $\overline{-}$ & $<<3.7$ \\
\hline 122 & $6: 40: 31.7$ & 9:30:08.4 & 1.00 & - & - & - & 3817 & - & - & - & $<2.4$ \\
\hline 123 & $6: 40: 31.7$ & $9: 38: 26.9$ & 1.00 & - & - & - & 3821 & - & - & - & $<2.4$ \\
\hline 124 & $6: 40: 31.8$ & 9:37:58.9 & 0.50 & - & $06403175+0937588$ & 2480 & 3825 & - & - & - & $<2.4$ \\
\hline 125 & $6: 40: 31.8$ & 9:32:03.4 & 1.00 & - & - & - & 3827 & - & - & - & $<2.0$ \\
\hline $126^{*}$ & $6: 40: 31.8$ & 9:33:29.4 & 0.50 & 10 & $06403181+0933293$ & 2482 & 3828 & - & - & - & 2.1 \\
\hline $127 *$ & $6: 40: 31.8$ & 9:36:00.6 & 0.50 & 11 & $06403183+0936006$ & 2484 & 3829 & - & - & - & 26.8 \\
\hline 128 & $6: 40: 31.9$ & $9: 38: 55.8$ & 0.67 & - & $06403186+0938557$ & 2486 & - & - & - & - & $<2.5$ \\
\hline $129 *$ & $6: 40: 31.9$ & $9: 32: 16.7$ & 2.84 & 12 & - & - & - & - & - & - & 3.8 \\
\hline 130 & $6: 40: 31.9$ & $9: 32: 02.6$ & 0.84 & - & $06403191+0932026$ & - & 3836 & - & - & - & $<2.0$ \\
\hline 131 & $6: 40: 31.9$ & $9: 28: 42.1$ & 1.00 & - & - & - & 3841 & - & - & - & $<2.5$ \\
\hline 132 & $6: 40: 31.9$ & $9: 28: 31.5$ & 0.50 & - & 06403194+0928315 & 2488 & 3842 & - & - & - & $<2.5$ \\
\hline 133 & $6: 40: 32.0$ & $9: 28: 02.5$ & 1.00 & - & - & - & 3848 & - & - & - & $<2.7$ \\
\hline 134 & $6: 40: 32.2$ & 9:30:04.8 & 0.50 & - & $06403216+0930047$ & - & 3856 & - & - & - & $<2.3$ \\
\hline 135 & $6: 40: 32.2$ & $9: 33: 50.0$ & 1.00 & - & - & - & 3859 & - & - & - & $<1.8$ \\
\hline 136 & $6: 40: 32.2$ & $9: 27: 40.3$ & 1.00 & - & - & - & 3862 & - & - & - & $<2.8$ \\
\hline 137 & $6: 40: 32.3$ & $9: 28: 37.8$ & 1.00 & - & - & - & 3865 & - & - & - & $<2.5$ \\
\hline 138 & $6: 40: 32.3$ & $9: 39: 52.0$ & 1.00 & - & - & - & 3866 & - & - & - & $<2.8$ \\
\hline 139 & $6: 40: 32.3$ & $9: 40: 23.8$ & 1.00 & - & - & - & 3869 & - & - & - & $<3.1$ \\
\hline 140 & $6: 40: 32.4$ & $9: 28: 37.0$ & 1.00 & - & - & - & 3875 & - & - & - & $<2.5$ \\
\hline 141 & $6: 40: 32.4$ & $9: 30: 50.7$ & 1.00 & - & - & - & 3876 & - & - & - & $<2.2$ \\
\hline 142 & $6: 40: 32.5$ & $9: 27: 35.1$ & 1.00 & - & - & - & 3877 & - & - & - & $<2.9$ \\
\hline 143 & $6: 40: 32.5$ & $9: 40: 15.7$ & 0.50 & - & $06403253+0940156$ & 2506 & 3883 & - & - & - & $<2.9$ \\
\hline 144 & $6: 40: 32.6$ & $9: 39: 28.1$ & 1.00 & - & - & - & 3886 & - & - & - & $<2.7$ \\
\hline 145 & $6: 40: 32.6$ & $9: 27: 28.5$ & 1.00 & - & - & - & 3888 & - & - & - & $<3.4$ \\
\hline 146 & $6: 40: 32.6$ & $9: 38: 25.6$ & 1.00 & - & - & - & 3889 & - & - & - & $<2.3$ \\
\hline $147 *$ & 6:40:32.6 & $9: 41: 52.4$ & 1.00 & 13 & - & - & 3892 & - & - & - & 7.0 \\
\hline 148 & $6: 40: 32.7$ & $9: 34: 51.5$ & 0.50 & - & $06403269+0934515$ & 2509 & 3897 & - & - & - & $<1.8$ \\
\hline 149 & $6: 40: 32.7$ & $9: 38: 32.5$ & 1.00 & - & - & - & 3895 & - & - & - & $<2.3$ \\
\hline 150 & $6: 40: 32.7$ & $9: 31: 18.7$ & 1.00 & - & - & - & 3898 & - & - & - & $<2.2$ \\
\hline 151 & $6: 40: 32.7$ & $9: 27: 19.5$ & 1.00 & - & - & - & 3901 & - & - & - & $<8.3$ \\
\hline 152 & $6: 40: 32.8$ & $9: 37: 41.0$ & 1.00 & - & - & - & 3905 & - & - & - & $<2.2$ \\
\hline 153 & $6: 40: 32.8$ & $9: 35: 20.0$ & 0.88 & - & $06403283+0935199$ & - & 3906 & - & - & - & $<1.9$ \\
\hline 154 & $6: 40: 32.9$ & $9: 41: 35.2$ & 0.50 & - & $06403285+0941352$ & - & 3914 & - & - & - & $<21.4$ \\
\hline 155 & 6:40:32.9 & $9: 39: 23.0$ & 0.50 & - & $06403285+0939230$ & 2512 & 3915 & - & - & - & $<2.8$ \\
\hline 156 & $6: 40: 32.9$ & $9: 40: 39.6$ & 1.00 & - & - & - & 3913 & - & - & - & $<3.1$ \\
\hline 157 & $6: 40: 32.9$ & $9: 32: 02.0$ & 0.50 & - & $06403289+0932020$ & 2513 & 3912 & - & - & - & $<1.9$ \\
\hline 158 & $6: 40: 33.0$ & $9: 36: 18.5$ & 0.50 & - & $06403298+0936184$ & - & 3921 & - & - & - & $<1.9$ \\
\hline 159 & $6: 40: 33.1$ & $9: 37: 46.5$ & 0.50 & - & $06403305+0937465$ & 2516 & 3928 & - & - & - & $<2.2$ \\
\hline 160 & $6: 40: 33.1$ & $9: 36: 49.3$ & 0.50 & - & $06403306+0936493$ & 2517 & 3927 & - & - & - & $<1.8$ \\
\hline 161 & $6: 40: 33.1$ & $9: 32: 42.2$ & 0.50 & - & $06403306+0932421$ & - & 3926 & - & - & - & $<1.8$ \\
\hline 162 & $6: 40: 33.1$ & $9: 41: 40.7$ & 1.00 & - & - & - & 3929 & - & - & - & $<9.9$ \\
\hline 163 & $6: 40: 33.1$ & $9: 40: 29.9$ & 1.00 & - & - & - & 3931 & - & - & - & $<3.0$ \\
\hline 164 & $6: 40: 33.1$ & 9:42:04.2 & 1.00 & - & - & - & 3932 & - & - & - & $<7.3$ \\
\hline 165 & $6: 40: 33.2$ & $9: 34: 48.9$ & 0.50 & - & $06403315+0934488$ & - & - & - & - & - & $<1.8$ \\
\hline 166 & $6: 40: 33.2$ & $9: 28: 24.2$ & 1.00 & - & - & - & 3936 & - & - & - & $<2.4$ \\
\hline 167 & $6: 40: 33.2$ & $9: 38: 45.8$ & 1.00 & - & - & - & 3937 & - & - & - & $<2.4$ \\
\hline 168 & $6: 40: 33.2$ & $9: 40: 36.5$ & 1.00 & - & - & - & 3938 & - & - & - & $<3.0$ \\
\hline 169 & $6: 40: 33.2$ & $9: 39: 48.2$ & 1.00 & - & - & - & 3939 & - & - & - & $<2.9$ \\
\hline 170 & $6: 40: 33.3$ & 9:39:37.0 & 0.50 & - & $06403325+0939369$ & 2523 & 3945 & - & - & - & $<2.8$ \\
\hline 171 & $6: 40: 33.3$ & $9: 30: 45.1$ & 0.50 & - & $06403327+0930450$ & - & 3943 & - & - & - & $<1.9$ \\
\hline 172 & $6: 40: 33.3$ & $9: 29: 42.6$ & 1.00 & - & - & - & 3944 & - & - & - & $<2.2$ \\
\hline 173 & $6: 40: 33.3$ & $9: 30: 03.7$ & 0.64 & - & $06403329+0930037$ & - & 3946 & - & - & - & $<2.1$ \\
\hline 174 & $6: 40: 33.4$ & $9: 30: 48.0$ & 1.00 & - & - & - & 3950 & - & - & - & $<1.9$ \\
\hline 175 & $6: 40: 33.5$ & $9: 32: 53.0$ & 1.00 & - & - & - & 3954 & - & - & - & $<1.6$ \\
\hline 176 & $6: 40: 33.5$ & $9: 40: 01.7$ & 1.00 & - & - & - & 3955 & - & - & - & $<3.0$ \\
\hline 177 & $6: 40: 33.5$ & 9:39:11.9 & 1.00 & - & - & - & 3957 & - & - & - & $<2.8$ \\
\hline 178 & $6: 40: 33.5$ & $9: 41: 52.0$ & 1.00 & - & - & - & 3958 & - & - & - & $<6.0$ \\
\hline 179 & $6: 40: 33.5$ & $9: 41: 49.7$ & 0.64 & - & $06403347+0941497$ & 2529 & 3963 & - & - & - & $<6.0$ \\
\hline 180 & $6: 40: 33.5$ & $9: 29: 27.4$ & 1.00 & - & - & - & 3959 & - & - & - & $<2.2$ \\
\hline
\end{tabular}


Table 3. (Continued)

\begin{tabular}{|c|c|c|c|c|c|c|c|c|c|c|c|}
\hline$\overline{\overline{\mathrm{N}}}$ & $\begin{array}{c}\mathrm{RA} \\
{[\mathrm{h} \mathrm{m} \mathrm{s}]}\end{array}$ & $\begin{array}{c}\text { Dec. } \\
{[\mathrm{d} \mathrm{m} \mathrm{s}]}\end{array}$ & $\begin{array}{c}\text { Id. rad. } \\
\text { ["] }\end{array}$ & $\overline{\mathrm{ACIS}}$ & $\overline{\text { 2Mass }}$ & Reb.+ & Lamm+ & Flacc+ & $\overline{\text { W56 }}$ & Dahm+ & $\begin{array}{l}\text { Ct. Rate. } \\
{\left[10^{-4} s^{-1}\right]}\end{array}$ \\
\hline$\overline{181}$ & $\overline{76: 40: 33.5}$ & $9: 40: 29.8$ & $\overline{1.00}$ & $=$ & 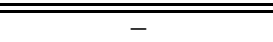 & $\overline{-1}$ & $\overline{\overline{3960}}$ & 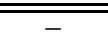 & $\overline{-}$ & $\overline{-}$ & $\overline{\angle<3.0}$ \\
\hline 182 & $6: 40: 33.5$ & $9: 40: 45.9$ & 1.00 & - & - & - & 3961 & - & - & - & $<3.1$ \\
\hline 183 & $6: 40: 33.5$ & $9: 29: 29.7$ & 1.00 & - & - & - & 3962 & - & - & - & $<2.2$ \\
\hline 184 & $6: 40: 33.5$ & $9: 42: 55.0$ & 0.50 & - & $06403349+0942550$ & 2531 & 3967 & _- & _- & - & $<18.5$ \\
\hline $185^{*}$ & $6: 40: 33.5$ & $9: 34: 58.6$ & 0.50 & 14 & $06403350+0934586$ & 2532 & 3966 & - & _- & - & 7.8 \\
\hline 186 & $6: 40: 33.5$ & $9: 41: 45.4$ & 1.00 & - & - & - & 3970 & - & - & - & $<6.0$ \\
\hline 187 & $6: 40: 33.5$ & $9: 34: 11.6$ & 0.50 & - & $06403354+0934115$ & - & 3969 & - & - & - & $<1.6$ \\
\hline $188^{*}$ & $6: 40: 33.6$ & $9: 33: 36.4$ & 0.50 & 15 & $06403357+0933363$ & 2537 & 3972 & - & - & 58 & 4.8 \\
\hline $189^{*}$ & $6: 40: 33.6$ & $9: 27: 28.3$ & 0.50 & - & $06403357+0927282$ & 2538 & 3973 & - & - & - & $<3.0$ \\
\hline 190 & $6: 40: 33.6$ & $9: 41: 28.1$ & 0.50 & - & $06403360+0941281$ & 2539 & 3974 & - & - & - & $<3.6$ \\
\hline 191 & $6: 40: 33.6$ & $9: 33: 59.6$ & 0.50 & - & $06403362+0933595$ & - & 3975 & - & - & - & $<1.6$ \\
\hline 192 & $6: 40: 33.6$ & $9: 27: 20.0$ & 1.00 & _- & - & - & 3977 & _- & _- & _- & $<8.6$ \\
\hline 193 & $6: 40: 33.7$ & $9: 31: 53.9$ & 1.00 & - & - & - & 3979 & - & - & - & $<1.7$ \\
\hline 194 & $6: 40: 33.7$ & $9: 30: 51.9$ & 0.50 & - & $06403369+0930518$ & - & 3981 & - & - & - & $<1.8$ \\
\hline 195 & $6: 40: 33.7$ & $9: 27: 24.8$ & 1.00 & - & - & - & 3982 & - & - & - & $<3.3$ \\
\hline 196 & $6: 40: 33.7$ & $9: 32: 25.0$ & 0.59 & - & $06403373+0932249$ & 2544 & 3983 & - & - & - & $<1.8$ \\
\hline 197 & $6: 40: 33.8$ & $9: 32: 57.0$ & 0.50 & - & $06403376+0932570$ & 2546 & 3985 & - & 62 & - & $<1.6$ \\
\hline 198 & $6: 40: 33.8$ & $9: 40: 50.4$ & 1.00 & - & - & - & 3987 & - & - & - & $<3.1$ \\
\hline 199 & $6: 40: 33.8$ & $9: 43: 11.5$ & 1.00 & - & _- & - & 3989 & _- & _- & _- & $<26.4$ \\
\hline 200 & $6: 40: 33.8$ & $9: 34: 33.1$ & 0.50 & - & $06403379+0934331$ & - & 3991 & - & - & - & $<1.7$ \\
\hline 201 & $6: 40: 33.8$ & $9: 41: 15.5$ & 0.50 & - & $06403380+0941154$ & 2553 & 3996 & - & 61 & - & $<3.3$ \\
\hline 202 & $6: 40: 33.8$ & $9: 42: 26.9$ & 0.50 & - & $06403380+0942269$ & 2552 & 3997 & - & - & - & $<4.1$ \\
\hline 203 & $6: 40: 33.8$ & $9: 38: 45.7$ & 1.00 & - & - & - & 3995 & - & - & - & $<2.3$ \\
\hline 204 & $6: 40: 33.9$ & $9: 41: 03.8$ & 1.00 & - & - & - & 4000 & - & - & - & $<3.2$ \\
\hline 205 & $6: 40: 33.9$ & $9: 30: 57.8$ & 1.00 & _- & _- & - & 4002 & _- & _- & _- & $<1.8$ \\
\hline 206 & $6: 40: 33.9$ & $9: 28: 13.3$ & 0.50 & _- & $06403394+0928133$ & _- & 4004 & _- & _- & _- & $<2.4$ \\
\hline 207 & $6: 40: 34.0$ & $9: 31: 15.0$ & 0.50 & - & $06403397+0931149$ & - & 4006 & - & - & - & $<1.8$ \\
\hline 208 & $6: 40: 34.0$ & $9: 27: 40.3$ & 1.00 & - & - & - & 4011 & - & - & - & $<2.8$ \\
\hline 209 & $6: 40: 34.0$ & $9: 41: 51.7$ & 1.00 & - & - & - & 4012 & - & - & - & $<3.7$ \\
\hline 210 & $6: 40: 34.0$ & $9: 37: 43.9$ & 1.00 & - & - & - & 4015 & - & - & - & $<2.0$ \\
\hline 211 & $6: 40: 34.1$ & $9: 35: 26.9$ & 0.50 & - & $06403407+0935269$ & - & 4016 & - & - & - & $<1.9$ \\
\hline 212 & $6: 40: 34.1$ & $9: 39: 52.0$ & 1.00 & - & - & - & 4018 & - & - & - & $<3.0$ \\
\hline 213 & $6: 40: 34.1$ & $9: 42: 52.1$ & 1.00 & - & _- & _- & 4020 & _- & _- & _- & $<16.3$ \\
\hline 214 & $6: 40: 34.2$ & $9: 41: 35.4$ & 1.00 & - & - & - & 4023 & - & - & - & $<3.4$ \\
\hline 215 & $6: 40: 34.2$ & $9: 29: 17.9$ & 0.50 & - & $06403416+0929178$ & 2563 & 4022 & - & - & - & $<2.2$ \\
\hline 216 & $6: 40: 34.2$ & $9: 30: 47.8$ & 0.71 & - & $06403417+0930477$ & - & 4024 & - & - & - & $<1.8$ \\
\hline 217 & $6: 40: 34.2$ & $9: 39: 23.9$ & 0.69 & - & $06403423+0939239$ & - & 4029 & - & - & - & $<2.5$ \\
\hline 218 & $6: 40: 34.3$ & $9: 27: 55.6$ & 0.59 & - & $06403425+0927555$ & 2569 & 4031 & - & - & - & $<2.7$ \\
\hline 219 & $6: 40: 34.3$ & $9: 38: 51.7$ & 0.50 & - & $06403430+0938517$ & 2572 & 4034 & - & 63 & - & $<2.3$ \\
\hline $220 *$ & $6: 40: 34.3$ & $9: 39: 17.7$ & 3.20 & 16 & - & - & - & - & - & - & 3.4 \\
\hline 221 & $6: 40: 34.3$ & $9: 30: 38.4$ & 0.50 & - & $06403431+0930384$ & - & 4036 & - & - & - & $<1.8$ \\
\hline 222 & $6: 40: 34.3$ & 9:33:01.1 & 0.50 & - & $06403432+0933011$ & - & 4037 & - & - & - & $<1.5$ \\
\hline 223 & $6: 40: 34.4$ & 9:40:40.9 & 0.78 & - & $06403436+0940409$ & 2577 & 4043 & - & - & - & $<3.0$ \\
\hline 224 & $6: 40: 34.4$ & $9: 37: 17.2$ & 1.00 & - & - & - & 4039 & - & - & - & $<1.8$ \\
\hline 225 & $6: 40: 34.4$ & 9:43:58.0 & 1.00 & - & - & - & 4041 & - & - & - & $<17.0$ \\
\hline 226 & $6: 40: 34.4$ & $9: 29: 53.5$ & 1.00 & - & - & - & 4042 & - & - & - & $<2.1$ \\
\hline 227 & $6: 40: 34.4$ & $9: 42: 06.8$ & 1.00 & - & _- & - & 4044 & - & - & - & $<3.7$ \\
\hline 228 & $6: 40: 34.4$ & $9: 31: 07.1$ & 0.50 & - & $06403442+0931070$ & 2578 & 4045 & - & - & - & $<1.7$ \\
\hline 229 & $6: 40: 34.5$ & $9: 31: 53.7$ & 1.00 & - & - & - & 4047 & - & - & - & $<1.6$ \\
\hline $230^{*}$ & $6: 40: 34.5$ & $9: 35: 18.3$ & 0.50 & 17 & $06403446+0935182$ & 2579 & 4048 & - & - & 63 & 15.6 \\
\hline 231 & $6: 40: 34.5$ & 9:30:10.0 & 1.00 & - & - & - & 4049 & - & - & - & $<2.0$ \\
\hline 232 & $6: 40: 34.6$ & $9: 42: 40.6$ & 1.00 & - & - & - & 4054 & - & - & - & $<4.0$ \\
\hline 233 & $6: 40: 34.6$ & $9: 42: 22.3$ & 1.00 & - & - & - & 4056 & - & - & - & $<3.7$ \\
\hline 234 & $6: 40: 34.6$ & $9: 30: 20.9$ & 0.50 & - & $06403457+0930208$ & 2583 & 4058 & - & - & - & $<1.9$ \\
\hline 235 & $6: 40: 34.6$ & $9: 29: 14.6$ & 0.50 & - & $06403458+0929145$ & 2584 & 4059 & - & - & - & $<2.2$ \\
\hline 236 & $6: 40: 34.6$ & $9: 39: 18.6$ & 1.00 & - & - & - & 4060 & - & - & - & $<2.4$ \\
\hline 237 & $6: 40: 34.6$ & $9: 43: 04.3$ & 1.00 & - & - & - & 4061 & - & - & - & $<5.0$ \\
\hline 238 & $6: 40: 34.6$ & $9: 39: 21.7$ & 1.00 & - & - & - & 4063 & - & - & - & $<2.4$ \\
\hline 239 & $6: 40: 34.7$ & $9: 28: 54.8$ & 1.00 & - & - & - & 4064 & - & - & - & $<2.3$ \\
\hline 240 & $6: 40: 34.7$ & $9: 40: 15.6$ & 1.00 & - & - & - & 4065 & - & - & - & $<3.2$ \\
\hline
\end{tabular}


Table 3. (Continued)

\begin{tabular}{|c|c|c|c|c|c|c|c|c|c|c|c|}
\hline$\overline{\mathrm{N}}$ & $\begin{array}{c}\text { RA } \\
{[\mathrm{h} \mathrm{m} \mathrm{s}]}\end{array}$ & $\begin{array}{c}\text { Dec. } \\
{[\mathrm{d} \mathrm{m} \mathrm{s}]}\end{array}$ & $\begin{array}{c}\text { Id. rad. } \\
\text { ["] }\end{array}$ & $\overline{\mathrm{ACIS}}$ & $\overline{2 \text { 2Mass }}$ & Reb.+ & Lamm+ & Flacc+ & $\overline{\mathrm{W} 56}$ & $\overline{\text { Dahm+ }}$ & $\begin{array}{l}\text { Ct. Rate. } \\
{\left[10^{-4} s^{-1}\right]}\end{array}$ \\
\hline$\overline{241}$ & $6: 40: 34.7$ & $9: 37: 10.4$ & $\overline{1.00}$ & $\overline{-1}$ & $\overline{-1}$ & $\overline{-}$ & $\overline{4066}$ & $\overline{-}$ & $\overline{-1}$ & $\overline{-}$ & $\overline{<<1.7}$ \\
\hline 242 & $6: 40: 34.8$ & $9: 32: 46.3$ & 0.50 & - & $06403479+0932463$ & - & 4068 & - & - & - & $<1.5$ \\
\hline 243 & $6: 40: 34.8$ & 9:40:33.1 & 0.96 & - & $06403481+0940330$ & 2592 & 4071 & - & - & - & $<3.0$ \\
\hline 244 & $6: 40: 34.9$ & $9: 37: 26.7$ & 0.68 & _- & $06403492+0937266$ & - & 4080 & - & _- & _- & $<1.8$ \\
\hline 245 & $6: 40: 34.9$ & $9: 42: 50.5$ & 1.00 & - & - & _- & 4077 & - & - & _- & $<4.1$ \\
\hline 246 & $6: 40: 35.0$ & $9: 33: 14.7$ & 0.60 & - & $06403496+0933146$ & _- & - & - & - & - & $<1.4$ \\
\hline 247 & $6: 40: 35.0$ & $9: 27: 52.2$ & 0.50 & - & $06403497+0927521$ & - & 4081 & - & - & - & $<2.7$ \\
\hline 248 & $6: 40: 35.0$ & $9: 35: 30.3$ & 0.50 & - & $06403499+0935303$ & - & 4083 & - & - & - & $<2.4$ \\
\hline $249 *$ & $6: 40: 35.0$ & 9:39:54.9 & 4.34 & 18 & - & - & - & - & - & - & 3.2 \\
\hline 250 & $6: 40: 35.1$ & $9: 28: 58.6$ & 0.50 & - & $06403508+0928586$ & - & 4088 & - & - & - & $<2.2$ \\
\hline 251 & $6: 40: 35.1$ & $9: 41: 52.4$ & 1.00 & - & - & - & 4089 & - & - & _- & $<3.3$ \\
\hline 252 & $6: 40: 35.1$ & $9: 41: 32.1$ & 1.00 & _- & - & _- & 4091 & - & - & _- & $<3.1$ \\
\hline 253 & $6: 40: 35.1$ & $9: 43: 55.2$ & 1.00 & - & - & - & 4092 & - & - & - & $<8.2$ \\
\hline 254 & $6: 40: 35.2$ & 9:28:20.7 & 0.50 & - & $06403517+0928206$ & 2596 & 4094 & - & - & - & $<2.5$ \\
\hline 255 & $6: 40: 35.2$ & 9:29:56.9 & 0.50 & - & $06403519+0929569$ & 2598 & 4097 & - & - & - & $<2.0$ \\
\hline 256 & $6: 40: 35.2$ & $9: 38: 11.4$ & 0.50 & - & $06403521+0938113$ & 2600 & 4100 & - & - & - & $<1.8$ \\
\hline 257 & $6: 40: 35.2$ & $9: 42: 26.2$ & 1.00 & - & - & - & 4101 & - & - & - & $<3.7$ \\
\hline 258 & $6: 40: 35.3$ & $9: 41: 51.3$ & 1.00 & - & - & - & 4103 & - & - & - & $<3.3$ \\
\hline 259 & $6: 40: 35.3$ & 9:38:08.9 & 1.00 & _- & - & _- & 4106 & - & _- & _- & $<1.8$ \\
\hline 260 & $6: 40: 35.3$ & $9: 29: 11.4$ & 1.00 & - & - & - & 4107 & - & - & - & $<2.2$ \\
\hline 261 & $6: 40: 35.4$ & $9: 37: 46.5$ & 1.00 & - & - & - & 4109 & - & - & - & $<1.8$ \\
\hline 262 & $6: 40: 35.4$ & $9: 27: 30.5$ & 0.50 & - & $06403541+0927304$ & 2602 & 4111 & - & - & - & $<2.7$ \\
\hline 263 & $6: 40: 35.5$ & 9:39:59.4 & 1.00 & - & - & - & 4113 & - & - & - & $<3.0$ \\
\hline 264 & $6: 40: 35.5$ & $9: 41: 41.0$ & 1.00 & - & - & - & 4114 & - & - & - & $<3.2$ \\
\hline 265 & $6: 40: 35.5$ & $9: 43: 54.2$ & 0.50 & _- & $06403550+0943542$ & 2607 & 4117 & - & _- & _- & $<11.9$ \\
\hline $266^{*}$ & $6: 40: 35.5$ & $9: 42: 28.2$ & 0.50 & - & $06403550+0942281$ & 2608 & 4118 & _- & _- & _- & $<3.7$ \\
\hline 267 & $6: 40: 35.5$ & $9: 28: 45.7$ & 1.00 & - & - & - & 4119 & - & - & - & $<2.3$ \\
\hline 268 & $6: 40: 35.5$ & 9:34:20.1 & 0.50 & - & $06403553+0934200$ & - & - & - & - & - & $<1.4$ \\
\hline 269 & $6: 40: 35.6$ & $9: 28: 04.6$ & 1.00 & - & - & - & 4121 & - & - & - & $<2.6$ \\
\hline 270 & $6: 40: 35.7$ & 9:28:53.9 & 1.00 & - & - & - & 4127 & - & - & - & $<2.2$ \\
\hline 271 & $6: 40: 35.7$ & $9: 39: 47.4$ & 1.00 & - & - & - & 4129 & - & - & - & $<2.8$ \\
\hline 272 & $6: 40: 35.8$ & $9: 41: 33.8$ & 0.80 & _- & $06403583+0941337$ & 2611 & 4136 & - & _- & _- & $<3.0$ \\
\hline 273 & $6: 40: 35.9$ & $9: 35: 33.4$ & 0.50 & - & $06403585+0935334$ & - & - & _- & _- & _- & $<3.1$ \\
\hline 274 & $6: 40: 35.9$ & $9: 27: 46.7$ & 1.00 & - & - & - & 4137 & - & - & - & $<2.7$ \\
\hline 275 & $6: 40: 35.9$ & $9: 38: 44.5$ & 1.00 & - & - & - & 4138 & - & - & - & $<2.0$ \\
\hline 276 & $6: 40: 35.9$ & $9: 27: 29.2$ & 1.00 & - & - & - & 4139 & - & - & - & $<2.7$ \\
\hline 277 & $6: 40: 35.9$ & $9: 42: 28.2$ & 1.00 & - & - & - & 4143 & - & - & - & $<3.7$ \\
\hline 278 & $6: 40: 36.0$ & $9: 41: 46.0$ & 1.00 & - & - & - & 4150 & - & - & - & $<3.2$ \\
\hline 279 & $6: 40: 36.0$ & $9: 42: 44.6$ & 1.00 & - & - & _- & 4151 & _- & - & - & $<4.0$ \\
\hline 280 & $6: 40: 36.0$ & $9: 42: 50.6$ & 1.00 & - & - & _- & 4152 & - & - & - & $<4.0$ \\
\hline 281 & $6: 40: 36.1$ & 9:41:58.4 & 0.68 & - & $06403606+0941584$ & - & 4149 & - & - & - & $<3.3$ \\
\hline 282 & $6: 40: 36.2$ & $9: 27: 19.2$ & 1.00 & - & - & - & 4158 & - & - & - & $<2.8$ \\
\hline $283^{*}$ & $6: 40: 36.2$ & 9:28:04.2 & 0.50 & 20 & $06403621+0928042$ & 2617 & 4159 & - & - & 68 & 3.3 \\
\hline $284 *$ & $6: 40: 36.2$ & 9:40:01.6 & 0.50 & 19 & $06403622+0940015$ & 2618 & 4161 & - & - & 69 & 8.9 \\
\hline 285 & $6: 40: 36.3$ & $9: 40: 51.1$ & 0.50 & - & $06403625+0940510$ & - & 4162 & - & - & - & $<3.3$ \\
\hline 286 & $6: 40: 36.3$ & $9: 27: 08.9$ & 0.89 & - & $06403628+0927089$ & _- & 4163 & _- & _- & _- & $<9.4$ \\
\hline $287 *$ & $6: 40: 36.3$ & $9: 40: 37.0$ & 8.92 & 21 & - & _- & - & - & - & _- & 3.3 \\
\hline 288 & $6: 40: 36.3$ & $9: 42: 58.9$ & 0.50 & - & $06403633+0942588$ & 2623 & 4167 & - & - & - & $<4.1$ \\
\hline $289 *$ & $6: 40: 36.4$ & 9:43:08.0 & 6.61 & 22 & - & - & - & - & - & - & 3.0 \\
\hline 290 & $6: 40: 36.4$ & $9: 38: 44.3$ & 1.00 & - & - & - & 4175 & - & - & - & $<1.9$ \\
\hline 291 & 6:40:36.4 & $9: 28: 50.4$ & 1.00 & - & - & - & 4178 & - & - & - & $<2.1$ \\
\hline 292 & $6: 40: 36.5$ & $9: 41: 18.1$ & 1.00 & - & - & - & 4179 & - & - & - & $<3.1$ \\
\hline 293 & $6: 40: 36.5$ & $9: 41: 04.2$ & 0.50 & - & $06403651+0941042$ & - & 4182 & - & - & - & $<2.8$ \\
\hline 294 & $6: 40: 36.6$ & $9: 40: 12.4$ & 1.00 & - & - & - & 4186 & - & - & - & $<3.0$ \\
\hline 295 & $6: 40: 36.6$ & $9: 28: 52.9$ & 1.00 & - & - & - & 4187 & - & - & - & $<2.0$ \\
\hline 296 & $6: 40: 36.6$ & $9: 27: 31.0$ & 1.00 & - & - & - & 4189 & - & - & - & $<2.7$ \\
\hline 297 & $6: 40: 36.6$ & $9: 28: 18.1$ & 1.00 & - & - & - & 4190 & - & - & - & $<2.4$ \\
\hline 298 & $6: 40: 36.7$ & $9: 42: 15.2$ & 1.00 & - & - & - & 4194 & - & - & - & $<3.4$ \\
\hline 299 & $6: 40: 36.7$ & $9: 41: 24.7$ & 0.50 & - & $06403671+0941246$ & 2638 & 4198 & - & - & - & $<3.9$ \\
\hline 300 & $6: 40: 36.7$ & 9:34:54.4 & 0.50 & - & $06403673+0934543$ & - & 4199 & - & - & - & $<1.4$ \\
\hline
\end{tabular}


Table 3. (Continued)

\begin{tabular}{|c|c|c|c|c|c|c|c|c|c|c|c|}
\hline$\overline{\mathrm{N}}$ & $\begin{array}{c}\mathrm{RA} \\
{[\mathrm{h} \mathrm{m} \mathrm{s}]}\end{array}$ & $\begin{array}{c}\text { Dec. } \\
{[\mathrm{d} \mathrm{m} \mathrm{s}]}\end{array}$ & $\begin{array}{c}\text { Id. rad. } \\
\text { ["] } \\
\end{array}$ & $\overline{\text { ACIS }}$ & 2Mass & Reb.+ & $\overline{\text { Lamm+ }}$ & Flacc+ & $\overline{\text { W56 }}$ & $\overline{\overline{\text { Dahm+ }}}$ & $\begin{array}{l}\text { Ct. Rate. } \\
{\left[10^{-4} s^{-1}\right]}\end{array}$ \\
\hline$\overline{301}$ & $6: 6: 40: 36.8$ & $9: 9: 29: 30.6$ & $\overline{0.81}$ & $\overline{-1}$ & "06403675+0929306 & $\overline{22639}$ & "4200 & $\overline{-1}$ & $\overline{-1}$ & 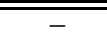 & ( <1.9 \\
\hline 302 & $6: 40: 36.8$ & $9: 29: 44.5$ & 1.00 & - & - & - & 4201 & - & - & - & $<1.9$ \\
\hline $303 *$ & $6: 40: 36.8$ & $9: 37: 30.5$ & 2.55 & 23 & - & - & - & - & - & - & 2.0 \\
\hline 304 & $6: 40: 36.8$ & $9: 39: 38.2$ & 0.59 & - & $06403679+0939382$ & - & 4206 & - & - & - & $<2.7$ \\
\hline $305 *$ & $6: 40: 36.8$ & $9: 41: 04.2$ & 0.50 & - & $06403679+0941042$ & - & 4207 & - & - & - & $<2.7$ \\
\hline 306 & $6: 40: 36.8$ & $9: 41: 02.8$ & 1.00 & - & - & - & 4208 & - & - & - & $<2.7$ \\
\hline 307 & $6: 40: 36.8$ & 9:43:02.8 & 0.50 & - & $06403684+0943027$ & 2641 & 4209 & - & - & - & $<4.1$ \\
\hline $308 *$ & $6: 40: 36.9$ & $9: 32: 37.5$ & 1.52 & 24 & - & - & - & - & - & - & 1.4 \\
\hline 309 & $6: 40: 36.9$ & $9: 39: 29.4$ & 1.00 & - & - & - & 4214 & - & - & - & $<2.5$ \\
\hline 310 & $6: 40: 36.9$ & $9: 40: 46.1$ & 1.00 & - & - & - & 4215 & - & - & - & $<3.3$ \\
\hline 311 & $6: 40: 36.9$ & $9: 27: 44.3$ & 0.50 & - & $06403693+0927442$ & 2645 & 4217 & - & - & - & $<2.7$ \\
\hline $312 *$ & $6: 40: 37.0$ & 9:39:09.8 & 0.50 & 25 & $06403697+0939097$ & - & 4220 & - & - & 74 & 22.1 \\
\hline 313 & $6: 40: 37.0$ & $9: 40: 60.0$ & 0.50 & - & $06403698+0940599$ & - & 4221 & - & - & - & $<2.7$ \\
\hline 314 & $6: 40: 37.0$ & $9: 41: 36.3$ & 1.00 & - & - & - & 4222 & - & - & - & $<3.9$ \\
\hline 315 & $6: 40: 37.0$ & 9:27:09.6 & 1.00 & - & - & - & 4227 & - & - & - & $<3.7$ \\
\hline $316^{*}$ & $6: 40: 37.2$ & $9: 31: 09.9$ & 0.50 & 26 & $06403720+0931098$ & $2665 \dagger$ & 4235 & $20 \dagger$ & - & 76 & 19.4 \\
\hline 317 & $6: 40: 37.3$ & $9: 41: 17.0$ & 1.00 & $28 \dagger$ & - & - & 4238 & - & - & - & $<3.9$ \\
\hline 318 & $6: 40: 37.3$ & $9: 40: 20.6$ & 1.00 & - & - & - & 4242 & - & - & - & $<2.7$ \\
\hline 319 & $6: 40: 37.3$ & $9: 41: 24.0$ & 1.00 & $28 \dagger$ & - & - & 4244 & - & - & - & $<3.9$ \\
\hline 320 & $6: 40: 37.3$ & $9: 35: 42.6$ & 0.50 & - & $06403732+0935426$ & - & - & - & 69 & - & $<2.2$ \\
\hline $321 *$ & $6: 40: 37.3$ & 9:39:09.4 & 1.50 & - & - & 2664 & - & - & - & - & $<16.6$ \\
\hline 322 & $6: 40: 37.4$ & $9: 29: 01.2$ & 0.93 & - & $06403738+0929012$ & - & 4249 & - & - & - & $<2.0$ \\
\hline 323 & $6: 40: 37.4$ & $9: 40: 30.5$ & 1.00 & - & - & - & 4248 & - & - & - & $<2.7$ \\
\hline 324 & $6: 40: 37.5$ & $9: 38: 12.8$ & 1.00 & - & - & - & 4252 & - & - & - & $<1.7$ \\
\hline 325 & $6: 40: 37.5$ & $9: 40: 47.8$ & 1.00 & - & - & - & 4255 & - & - & - & $<2.7$ \\
\hline 326 & $6: 40: 37.5$ & $9: 41: 20.0$ & 1.00 & $28 \dagger$ & - & - & 4258 & - & - & - & $<3.9$ \\
\hline 327 & $6: 40: 37.5$ & $9: 36: 32.1$ & 1.00 & - & - & - & 4260 & - & - & - & $<1.3$ \\
\hline 328 & $6: 40: 37.5$ & $9: 42: 33.3$ & 1.00 & - & - & - & 4261 & - & - & - & $<3.5$ \\
\hline 329 & $6: 40: 37.6$ & $9: 41: 44.2$ & 0.50 & - & $06403756+0941441$ & - & 4263 & - & - & - & $<3.5$ \\
\hline 330 & $6: 40: 37.6$ & $9: 42: 15.7$ & 0.50 & - & $06403759+0942156$ & 2660 & 4266 & - & - & - & $<3.3$ \\
\hline 331 & $6: 40: 37.6$ & $9: 31: 10.4$ & 0.61 & - & $06403759+0931103$ & $2665 \dagger$ & 4262 & $20 \dagger$ & - & - & $<5.0$ \\
\hline $332 *$ & $6: 40: 37.7$ & $9: 39: 39.8$ & 0.50 & 27 & $06403768+0939397$ & 2663 & 4271 & - & - & 77 & 11.3 \\
\hline 333 & $6: 40: 37.7$ & $9: 27: 58.0$ & 1.00 & - & - & - & 4270 & - & - & - & $<2.5$ \\
\hline 334 & $6: 40: 37.7$ & $9: 37: 28.5$ & 0.50 & - & $06403772+0937285$ & - & 4272 & - & - & - & $<1.6$ \\
\hline 335 & $6: 40: 37.7$ & $9: 41: 17.0$ & 1.00 & $28 \dagger$ & - & - & 4273 & - & - & - & $<3.9$ \\
\hline 336 & $6: 40: 37.8$ & $9: 34: 12.9$ & 0.50 & - & $06403775+0934128$ & - & 4274 & - & - & - & $<1.2$ \\
\hline 337 & $6: 40: 37.8$ & $9: 40: 10.6$ & 0.50 & - & $06403779+0940105$ & 2678 & 4278 & - & - & - & $<2.6$ \\
\hline 338 & $6: 40: 37.8$ & $9: 31: 18.8$ & 0.50 & - & $06403783+0931187$ & - & 4279 & - & - & - & $<1.5$ \\
\hline $339 *$ & $6: 40: 37.9$ & $9: 35: 56.7$ & 0.50 & 29 & $06403786+0935567$ & - & 4280 & - & - & - & 30.3 \\
\hline $340 *$ & $6: 40: 37.9$ & $9: 34: 54.0$ & 0.50 & 30 & $06403787+0934540$ & - & 4282 & 23 & - & 79 & 71.0 \\
\hline 341 & $6: 40: 37.9$ & $9: 29: 17.2$ & 1.00 & - & - & - & 4284 & - & - & - & $<1.9$ \\
\hline 342 & $6: 40: 37.9$ & $9: 43: 21.4$ & 0.50 & - & $06403788+0943213$ & - & 4285 & - & - & - & $<3.9$ \\
\hline 343 & $6: 40: 37.9$ & $9: 37: 41.5$ & 1.00 & - & - & - & 4288 & - & - & - & $<1.6$ \\
\hline 344 & $6: 40: 37.9$ & $9: 40: 22.2$ & 0.50 & - & $06403794+0940222$ & - & 4290 & - & - & - & $<2.5$ \\
\hline 345 & $6: 40: 37.9$ & $9: 39: 24.0$ & 0.50 & - & $06403794+0939239$ & 2684 & 4292 & - & - & - & $<2.2$ \\
\hline 346 & $6: 40: 38.0$ & $9: 30: 49.0$ & 1.00 & - & - & - & 4293 & - & - & - & $<1.5$ \\
\hline 347 & $6: 40: 38.0$ & 9:43:04.7 & 1.00 & - & - & - & 4294 & - & - & - & $<3.9$ \\
\hline 348 & $6: 40: 38.0$ & $9: 29: 50.7$ & 1.00 & - & - & - & 4297 & - & - & - & $<4.1$ \\
\hline 349 & $6: 40: 38.0$ & $9: 32: 49.2$ & 0.50 & - & $06403803+0932491$ & 2686 & 4300 & - & - & - & $<1.3$ \\
\hline $350 *$ & $6: 40: 38.1$ & $9: 31: 34.7$ & 1.96 & 31 & - & - & - & - & - & - & 1.6 \\
\hline 351 & $6: 40: 38.1$ & $9: 42: 22.5$ & 1.00 & - & - & - & 4305 & - & - & - & $<3.4$ \\
\hline 352 & $6: 40: 38.2$ & $9: 28: 30.5$ & 0.66 & - & $06403817+0928304$ & - & 4308 & - & - & - & $<2.1$ \\
\hline 353 & $6: 40: 38.2$ & $9: 39: 16.1$ & 1.00 & - & - & - & 4309 & - & - & - & $<2.2$ \\
\hline $354 *$ & $6: 40: 38.2$ & $9: 29: 52.5$ & 0.50 & 32 & $06403819+0929524$ & 2692 & 4313 & - & - & 80 & 6.8 \\
\hline 355 & $6: 40: 38.2$ & $9: 42: 21.3$ & 1.00 & - & - & - & 4314 & - & - & - & $<3.3$ \\
\hline 356 & $6: 40: 38.2$ & $9: 27: 01.6$ & 1.00 & - & - & - & 4315 & - & - & - & $<4.6$ \\
\hline 357 & $6: 40: 38.2$ & $9: 28: 19.9$ & 0.50 & - & $06403823+0928198$ & 2693 & 4316 & - & - & - & $<2.2$ \\
\hline 358 & $6: 40: 38.3$ & $9: 42: 51.6$ & 1.00 & - & - & - & 4317 & - & - & - & $<3.6$ \\
\hline 359 & $6: 40: 38.3$ & $9: 28: 59.6$ & 0.71 & - & $06403829+0928595$ & - & 4321 & - & - & - & $<2.0$ \\
\hline 360 & $6: 40: 38.3$ & $9: 42: 50.3$ & 0.50 & - & $06403829+0942502$ & 2695 & 4322 & - & - & - & $<3.5$ \\
\hline
\end{tabular}


Table 3. (Continued)

\begin{tabular}{|c|c|c|c|c|c|c|c|c|c|c|c|}
\hline$\overline{\mathrm{N}}$ & $\begin{array}{c}\text { RA } \\
{[\mathrm{h} \mathrm{m} \mathrm{s}]}\end{array}$ & $\begin{array}{c}\text { Dec. } \\
{[\mathrm{d} \mathrm{m} \mathrm{s}]}\end{array}$ & $\begin{array}{c}\text { Id. rad. } \\
\text { ["] }\end{array}$ & $\overline{\mathrm{ACIS}}$ & $\overline{2 \text { 2Mass }}$ & Reb.+ & Lamm+ & Flacc+ & $\overline{\mathrm{W} 56}$ & $\overline{\text { Dahm+ }}$ & $\begin{array}{l}\text { Ct. Rate. } \\
{\left[10^{-4} s^{-1}\right]}\end{array}$ \\
\hline$\overline{361}$ & $60: 40: 38.3$ & $9: 27: 25.0$ & $\overline{1.00}$ & $\overline{-1}$ & $\overline{-1}$ & $\overline{-}$ & $\overline{43320}$ & $\overline{-}$ & $\overline{-1}$ & $\overline{-}$ & $\overline{<<2.5}$ \\
\hline 362 & $6: 40: 38.3$ & $9: 29: 25.4$ & 1.00 & - & - & - & 4323 & - & - & - & $<1.8$ \\
\hline 363 & $6: 40: 38.3$ & $9: 29: 26.0$ & 0.50 & - & $06403834+0929260$ & 2699 & 4326 & - & - & - & $<1.8$ \\
\hline 364 & $6: 40: 38.4$ & $9: 41: 23.6$ & 1.00 & $28+$ & - & - & 4328 & - & _- & _- & $<3.9$ \\
\hline 365 & $6: 40: 38.4$ & $9: 33: 17.1$ & 1.00 & - & - & _- & 4339 & _- & - & _- & $<1.2$ \\
\hline 366 & $6: 40: 38.6$ & $9: 30: 21.5$ & 0.60 & - & $06403859+0930215$ & - & 4342 & - & - & - & $<1.6$ \\
\hline 367 & $6: 40: 38.6$ & $9: 39: 50.0$ & 1.00 & - & - & - & 4343 & - & - & - & $<2.4$ \\
\hline $368^{*}$ & $6: 40: 38.7$ & 9:36:57.0 & 0.50 & 33 & $06403867+0936569$ & 2709 & 4349 & 25 & - & 81 & 10.0 \\
\hline $369^{*}$ & $6: 40: 38.7$ & $9: 34: 34.3$ & 0.50 & 34 & $06403868+0934342$ & - & 4348 & - & - & - & 1.4 \\
\hline 370 & $6: 40: 38.8$ & $9: 38: 37.3$ & 1.00 & - & - & - & 4354 & - & - & - & $<1.7$ \\
\hline $371 *$ & $6: 40: 38.8$ & $9: 29: 48.1$ & 1.75 & 35 & - & - & - & - & - & - & 2.3 \\
\hline 372 & $6: 40: 38.9$ & $9: 40: 50.9$ & 0.61 & _- & $06403885+0940508$ & _- & 4358 & - & - & _- & $<2.4$ \\
\hline 373 & $6: 40: 38.9$ & $9: 39: 46.9$ & 0.50 & - & $06403890+0939469$ & - & 4362 & - & - & - & $<2.3$ \\
\hline 374 & $6: 40: 38.9$ & $9: 38: 49.0$ & 1.00 & - & - & - & 4363 & - & - & - & $<1.8$ \\
\hline $375^{*}$ & $6: 40: 39.0$ & 9:35:60.0 & 0.50 & 36 & $06403902+0935599$ & 2714 & 4367 & - & - & - & 15.9 \\
\hline 376 & $6: 40: 39.1$ & $9: 33: 15.6$ & 0.50 & - & $06403913+0933156$ & - & - & - & - & - & $<1.1$ \\
\hline 377 & $6: 40: 39.1$ & 9:29:36.6 & 1.00 & - & - & - & 4372 & - & - & - & $<1.8$ \\
\hline 378 & $6: 40: 39.2$ & $9: 35: 27.3$ & 0.50 & - & $06403915+0935273$ & - & - & - & - & - & $<2.4$ \\
\hline 379 & $6: 40: 39.2$ & $9: 32: 39.9$ & 0.50 & _- & $06403917+0932398$ & _- & - & - & _- & _- & $<1.2$ \\
\hline 380 & $6: 40: 39.2$ & $9: 43: 40.9$ & 0.50 & - & $06403919+0943409$ & - & 4383 & - & - & - & $<5.6$ \\
\hline 381 & $6: 40: 39.2$ & 9:41:47.9 & 0.50 & - & $06403919+0941479$ & 2720 & 4381 & - & - & - & $<3.0$ \\
\hline 382 & $6: 40: 39.2$ & $9: 28: 17.7$ & 1.00 & - & - & - & 4382 & - & - & - & $<2.2$ \\
\hline $383 *$ & $6: 40: 39.2$ & 9:29:30.4 & 1.91 & 37 & - & - & - & - & - & - & 2.2 \\
\hline 384 & $6: 40: 39.3$ & $9: 31: 28.1$ & 1.00 & - & - & - & 4387 & - & - & - & $<1.4$ \\
\hline 385 & $6: 40: 39.3$ & $9: 36: 14.0$ & 0.50 & _- & $06403928+0936140$ & _- & - & - & _- & _- & $<1.3$ \\
\hline 386 & $6: 40: 39.3$ & $9: 40: 18.9$ & 1.00 & - & _- & _- & 4388 & _- & _- & _- & $<2.3$ \\
\hline 387 & $6: 40: 39.3$ & 9:31:29.2 & 0.50 & - & $06403933+0931292$ & 2724 & 4390 & - & - & - & $<1.4$ \\
\hline $388^{*}$ & $6: 40: 39.3$ & $9: 34: 45.6$ & 0.50 & 38 & $06403934+0934455$ & 2725 & 4392 & - & - & 85 & 3.8 \\
\hline 389 & $6: 40: 39.4$ & $9: 30: 34.0$ & 1.00 & - & - & - & 4395 & - & - & - & $<1.5$ \\
\hline 390 & $6: 40: 39.4$ & 9:43:05.1 & 1.00 & - & - & - & 4396 & - & - & - & $<3.5$ \\
\hline 391 & $6: 40: 39.4$ & $9: 42: 34.3$ & 1.00 & - & - & - & 4399 & - & - & - & $<3.3$ \\
\hline 392 & $6: 40: 39.5$ & $9: 42: 56.3$ & 0.50 & _- & $06403947+0942563$ & 2729 & 4403 & - & _- & _- & $<3.5$ \\
\hline 393 & $6: 40: 39.5$ & $9: 40: 56.2$ & 0.52 & - & $06403952+0940561$ & - & 4408 & _- & _- & _- & $<2.4$ \\
\hline 394 & $6: 40: 39.5$ & 9:34:11.4 & 0.53 & - & $06403953+0934113$ & - & - & - & - & - & $<1.1$ \\
\hline 395 & $6: 40: 39.5$ & $9: 42: 20.6$ & 1.00 & - & - & - & 4410 & - & - & - & $<3.2$ \\
\hline 396 & $6: 40: 39.6$ & $9: 28: 12.4$ & 1.00 & - & - & - & 4411 & - & - & - & $<2.2$ \\
\hline 397 & $6: 40: 39.6$ & $9: 29: 41.8$ & 0.50 & - & $06403957+0929417$ & 2733 & 4413 & - & - & - & $<1.8$ \\
\hline 398 & 6:40:39.6 & $9: 41: 35.1$ & 1.00 & - & - & - & 4414 & - & - & - & $<2.8$ \\
\hline 399 & $6: 40: 39.6$ & $9: 27: 20.1$ & 1.00 & - & - & _- & 4418 & _- & - & - & $<2.3$ \\
\hline 400 & $6: 40: 39.7$ & $9: 28: 37.8$ & 1.00 & - & - & _- & 4420 & - & - & - & $<1.9$ \\
\hline 401 & $6: 40: 39.7$ & 9:42:09.7 & 1.00 & - & - & - & 4422 & - & - & - & $<3.1$ \\
\hline 402 & $6: 40: 39.8$ & $9: 28: 35.0$ & 1.00 & - & - & - & 4424 & - & - & - & $<1.9$ \\
\hline 403 & $6: 40: 39.8$ & $9: 40: 10.5$ & 0.50 & - & $06403983+0940105$ & 2738 & 4427 & - & - & - & $<2.2$ \\
\hline 404 & $6: 40: 40.0$ & $9: 35: 42.7$ & 0.51 & - & $06403996+0935426$ & - & - & - & - & - & $<1.2$ \\
\hline 405 & $6: 40: 40.0$ & $9: 27: 14.2$ & 1.00 & - & - & - & 4436 & - & - & - & $<2.3$ \\
\hline 406 & $6: 40: 40.0$ & $9: 38: 31.2$ & 1.00 & - & - & - & 4437 & - & - & - & $<1.6$ \\
\hline 407 & $6: 40: 40.0$ & $9: 27: 20.7$ & 1.00 & _- & - & - & 4441 & - & - & _- & $<2.3$ \\
\hline 408 & $6: 40: 40.0$ & $9: 42: 32.0$ & 1.00 & - & - & - & 4442 & - & - & - & $<3.1$ \\
\hline $409 *$ & $6: 40: 40.1$ & 9:35:02.9 & 0.50 & 39 & $06404005+0935029$ & 2742 & 4443 & $26 \dagger$ & 77 & 87 & 26.4 \\
\hline 410 & $6: 40: 40.1$ & $9: 42: 07.2$ & 1.00 & - & - & - & 4444 & - & - & - & $<3.0$ \\
\hline 411 & 6:40:40.1 & 9:43:47.1 & 0.50 & - & $06404006+0943470$ & - & 4446 & - & - & - & $<9.5$ \\
\hline 412 & 6:40:40.1 & 9:41:32.9 & 1.00 & - & - & - & 4447 & - & - & - & $<2.9$ \\
\hline 413 & $6: 40: 40.1$ & $9: 28: 28.5$ & 1.00 & - & - & - & 4449 & - & - & - & $<2.0$ \\
\hline 414 & $6: 40: 40.2$ & $9: 43: 41.4$ & 0.50 & - & $06404016+0943413$ & 2746 & 4451 & - & - & - & $<4.9$ \\
\hline 415 & $6: 40: 40.2$ & $9: 41: 49.5$ & 0.62 & - & $06404017+0941495$ & 2747 & 4450 & - & - & - & $<3.0$ \\
\hline 416 & $6: 40: 40.2$ & 9:43:04.3 & 0.50 & - & $06404021+0943042$ & 2748 & 4452 & - & - & - & $<3.4$ \\
\hline 417 & $6: 40: 40.3$ & $9: 40: 41.2$ & 1.00 & - & - & - & 4455 & - & - & - & $<2.3$ \\
\hline 418 & $6: 40: 40.3$ & 9:37:43.0 & 1.00 & - & - & - & 4457 & - & - & - & $<1.4$ \\
\hline 419 & $6: 40: 40.4$ & $9: 39: 35.7$ & 0.50 & - & $06404037+0939357$ & - & 4464 & - & - & - & $<1.9$ \\
\hline 420 & $6: 40: 40.4$ & $9: 41: 19.3$ & 1.00 & - & - & - & 4467 & - & - & - & $<2.7$ \\
\hline
\end{tabular}


Table 3. (Continued)

\begin{tabular}{|c|c|c|c|c|c|c|c|c|c|c|c|}
\hline$\overline{\mathrm{N}}$ & $\begin{array}{c}\text { RA } \\
{[\mathrm{h} \mathrm{m} \mathrm{s}]}\end{array}$ & $\begin{array}{c}\text { Dec. } \\
{[\mathrm{d} \mathrm{m} \mathrm{s}]} \\
\end{array}$ & $\begin{array}{c}\text { Id. rad. } \\
\text { ["] } \\
\end{array}$ & $\overline{\mathrm{ACIS}}$ & 2Mass & Reb.+ & $\overline{\text { Lamm+ }}$ & Flacc+ & $\overline{\text { W56 }}$ & Dahm+ & $\begin{array}{l}\text { Ct. Rate. } \\
{\left[10^{-4} s^{-1}\right]} \\
\end{array}$ \\
\hline $421^{*}$ & $\overline{60: 40: 40.4}$ & $9: 31: 34.8$ & $\overline{0.50}$ & $\overline{40}$ & $06404043+0931347$ & 2754 & 4468 & - & - & - & $\overline{1.9}$ \\
\hline $422 *$ & $6: 40: 40.5$ & $9: 38: 25.7$ & 2.22 & 42 & - & - & - & - & - & - & 2.7 \\
\hline $423 *$ & $6: 40: 40.5$ & $9: 35: 05.5$ & 0.50 & 41 & $06404050+0935055$ & - & 4474 & $26 \dagger$ & - & - & 2.1 \\
\hline 424 & $6: 40: 40.5$ & $9: 40: 17.9$ & 1.00 & - & - & - & 4476 & - & - & - & $<2.2$ \\
\hline $425^{*}$ & $6: 40: 40.5$ & 9:35:01.1 & 0.50 & 43 & $06404052+0935011$ & - & 4475 & $26 \dagger$ & - & - & 1.9 \\
\hline $426^{*}$ & $6: 40: 40.5$ & $9: 29: 19.8$ & 0.50 & - & $06404052+0929197$ & 2757 & 4477 & - & - & 90 & $<1.7$ \\
\hline 427 & $6: 40: 40.6$ & $9: 27: 49.3$ & 0.50 & - & $06404058+0927492$ & 2758 & 4480 & - & - & - & $<2.3$ \\
\hline 428 & $6: 40: 40.6$ & $9: 27: 07.3$ & 1.00 & - & - & - & 4482 & - & - & - & $<2.4$ \\
\hline 429 & $6: 40: 40.7$ & $9: 30: 29.5$ & 0.50 & - & $06404065+0930295$ & - & 4486 & - & - & - & $<1.5$ \\
\hline 430 & $6: 40: 40.7$ & $9: 31: 11.4$ & 1.00 & - & - & - & 4488 & - & - & - & $<1.4$ \\
\hline 431 & $6: 40: 40.7$ & $9: 42: 13.2$ & 0.59 & - & $06404069+0942131$ & 2762 & 4489 & - & - & - & $<3.1$ \\
\hline $432 *$ & $6: 40: 40.8$ & $9: 34: 26.9$ & 0.50 & 44 & $06404078+0934269$ & 2764 & 4490 & 27 & - & - & 1.3 \\
\hline 433 & $6: 40: 40.8$ & $9: 27: 39.0$ & 1.00 & - & - & - & 4494 & - & - & - & $<2.3$ \\
\hline 434 & $6: 40: 40.8$ & $9: 40: 20.0$ & 1.00 & - & - & - & 4497 & - & - & - & $<2.3$ \\
\hline 435 & $6: 40: 40.9$ & $9: 30: 40.4$ & 0.60 & - & $06404085+0930404$ & - & 4496 & - & - & - & $<1.4$ \\
\hline 436 & $6: 40: 40.9$ & $9: 29: 44.8$ & 0.50 & - & $06404086+0929448$ & 2767 & 4498 & - & - & - & $<1.6$ \\
\hline 437 & $6: 40: 40.9$ & $9: 38: 27.4$ & 0.71 & - & $06404088+0938274$ & - & 4499 & - & - & - & $<1.6$ \\
\hline 438 & $6: 40: 40.9$ & $9: 41: 21.7$ & 0.69 & - & $06404088+0941217$ & - & 4507 & - & - & - & $<2.7$ \\
\hline 439 & $6: 40: 40.9$ & $9: 42: 24.9$ & 1.00 & - & - & - & 4502 & - & - & - & $<3.1$ \\
\hline $440 *$ & $6: 40: 40.9$ & $9: 27: 59.5$ & 0.50 & 45 & $06404091+0927595$ & - & 4503 & - & - & 91 & 17.6 \\
\hline 441 & $6: 40: 40.9$ & 9:30:08.8 & 0.50 & - & $06404093+0930088$ & - & 4504 & - & - & - & $<1.5$ \\
\hline $442 *$ & $6: 40: 41.0$ & $9: 27: 54.3$ & 0.50 & - & $06404100+0927543$ & 2771 & 4511 & - & - & 92 & $<11.2$ \\
\hline 443 & $6: 40: 41.0$ & $9: 43: 12.7$ & 0.50 & - & $06404103+0943127$ & - & 4516 & - & - & - & $<3.4$ \\
\hline $444 *$ & $6: 40: 41.1$ & $9: 33: 57.9$ & 0.50 & 46 & $06404114+0933578$ & 2776 & 4522 & - & 79 & 94 & 1.1 \\
\hline 445 & $6: 40: 41.2$ & $9: 27: 23.5$ & 0.50 & - & $06404118+0927235$ & - & 4534 & - & - & - & $<2.3$ \\
\hline $446^{*}$ & $6: 40: 41.2$ & $9: 31: 28.7$ & 0.50 & 47 & $06404119+0931287$ & - & 4529 & - & - & - & 1.5 \\
\hline 447 & $6: 40: 41.2$ & $9: 27: 21.1$ & 1.00 & - & - & - & 4531 & - & - & - & $<2.4$ \\
\hline 448 & $6: 40: 41.2$ & $9: 30: 07.2$ & 0.50 & - & $06404120+0930072$ & 2779 & 4533 & - & - & - & $<1.5$ \\
\hline 449 & $6: 40: 41.2$ & $9: 28: 16.3$ & 1.00 & - & - & - & 4535 & - & - & - & $<2.0$ \\
\hline 450 & $6: 40: 41.2$ & $9: 38: 37.5$ & 1.00 & - & - & - & 4536 & - & - & - & $<1.6$ \\
\hline 451 & $6: 40: 41.3$ & $9: 37: 53.2$ & 0.50 & - & $06404130+0937532$ & - & 4539 & - & - & - & $<1.3$ \\
\hline $452 *$ & $6: 40: 41.3$ & $9: 39: 09.5$ & 2.14 & 48 & - & - & - & - & - & - & 3.9 \\
\hline 453 & $6: 40: 41.4$ & $9: 28: 04.4$ & 1.00 & - & - & - & 4548 & - & - & - & $<2.2$ \\
\hline 454 & $6: 40: 41.4$ & $9: 31: 31.5$ & 0.50 & - & $06404144+0931314$ & 2790 & 4555 & - & - & - & $<1.3$ \\
\hline 455 & $6: 40: 41.5$ & $9: 27: 30.4$ & 1.00 & - & - & - & 4556 & - & - & - & $<2.4$ \\
\hline 456 & $6: 40: 41.5$ & $9: 27: 37.1$ & 0.79 & - & $06404151+0927370$ & - & 4557 & - & - & - & $<2.3$ \\
\hline 457 & 6:40:41.6 & $9: 40: 14.7$ & 0.50 & - & $06404163+0940146$ & - & 4566 & - & - & - & $<2.2$ \\
\hline 458 & $6: 40: 41.6$ & $9: 32: 20.6$ & 10.12 & - & - & - & - & 30 & - & - & $<1.2$ \\
\hline 459 & $6: 40: 41.6$ & $9: 27: 19.9$ & 1.00 & - & - & - & 4565 & - & - & - & $<2.4$ \\
\hline $460 *$ & $6: 40: 41.6$ & $9: 31: 43.2$ & 0.50 & 49 & $06404164+0931431$ & 2793 & 4568 & - & - & 102 & 2.0 \\
\hline 461 & $6: 40: 41.8$ & $9: 26: 55.9$ & 0.50 & - & $06404176+0926559$ & 2800 & 4572 & - & - & - & $<2.8$ \\
\hline $462 *$ & $6: 40: 41.8$ & $9: 41: 38.6$ & 0.50 & 50 & $06404184+0941386$ & - & 4578 & - & - & 106 & 3.7 \\
\hline 463 & $6: 40: 41.9$ & 9:40:01.4 & 1.00 & - & - & - & 4581 & - & - & - & $<2.2$ \\
\hline 464 & $6: 40: 41.9$ & $9: 42: 24.0$ & 0.50 & - & $06404193+0942240$ & - & 4583 & - & - & - & $<2.8$ \\
\hline 465 & $6: 40: 41.9$ & $9: 35: 48.6$ & 0.57 & - & $06404193+0935486$ & 2804 & 4585 & - & - & - & $<1.0$ \\
\hline 466 & $6: 40: 42.1$ & $9: 31: 41.8$ & 0.50 & 51 & $06404213+0931418$ & - & 4595 & - & - & - & 3.1 \\
\hline 467 & $6: 40: 42.2$ & $9: 41: 53.1$ & 0.89 & - & $06404217+0941530$ & - & 4596 & - & - & - & $<2.9$ \\
\hline $468^{*}$ & $6: 40: 42.2$ & $9: 33: 37.4$ & 0.50 & 52 & $06404218+0933374$ & 2811 & 4598 & - & 84 & 107 & 19.5 \\
\hline 469 & $6: 40: 42.2$ & $9: 30: 57.6$ & 1.00 & - & - & - & 4599 & - & - & - & $<1.3$ \\
\hline $470^{*}$ & $6: 40: 42.2$ & $9: 40: 11.1$ & 0.50 & 53 & $06404221+0940110$ & 2817 & 4602 & $32 \dagger$ & - & 108 & 55.5 \\
\hline 471 & $6: 40: 42.2$ & 9:37:01.4 & 0.50 & - & $06404222+0937014$ & - & 4603 & - & - & - & $<1.2$ \\
\hline 472 & $6: 40: 42.2$ & $9: 38: 38.9$ & 0.50 & - & $06404223+0938389$ & - & - & - & - & - & $<1.6$ \\
\hline 473 & $6: 40: 42.2$ & $9: 42: 48.4$ & 0.50 & - & $06404223+0942483$ & - & - & - & - & - & $<3.1$ \\
\hline $474 *$ & $6: 40: 42.3$ & $9: 39: 21.3$ & 0.50 & 54 & $06404228+0939212$ & - & - & 31 & 83 & - & 75.5 \\
\hline 475 & $6: 40: 42.3$ & $9: 37: 57.2$ & 1.00 & - & - & - & 4608 & - & - & - & $<1.2$ \\
\hline $476^{*}$ & $6: 40: 42.3$ & $9: 34: 25.0$ & 0.50 & 55 & $06404232+0934250$ & 2819 & 4610 & - & - & 109 & 11.1 \\
\hline 477 & $6: 40: 42.4$ & $9: 32: 45.4$ & 0.56 & - & $06404235+0932454$ & - & - & - & - & - & $<1.0$ \\
\hline 478 & $6: 40: 42.4$ & $9: 27: 21.9$ & 0.50 & - & $06404237+0927218$ & 2820 & 4613 & - & - & - & $<2.3$ \\
\hline $479^{*}$ & $6: 40: 42.4$ & $9: 32: 20.6$ & 0.50 & 56 & $06404244+0932206$ & 2822 & 4615 & - & - & 110 & 32.6 \\
\hline 480 & $6: 40: 42.5$ & $9: 31: 26.6$ & 0.50 & - & $06404246+0931266$ & - & 4617 & - & - & - & $<1.2$ \\
\hline
\end{tabular}


Table 3. (Continued)

\begin{tabular}{|c|c|c|c|c|c|c|c|c|c|c|c|}
\hline$\overline{\mathrm{N}}$ & $\begin{array}{c}\mathrm{RA} \\
{[\mathrm{h} \mathrm{m} \mathrm{s}]}\end{array}$ & $\begin{array}{c}\text { Dec. } \\
{[\mathrm{d} \mathrm{m} \mathrm{s}]}\end{array}$ & $\begin{array}{c}\text { Id. rad. } \\
\text { ["] }\end{array}$ & $\overline{\mathrm{ACIS}}$ & 2Mass & Reb.+ & Lamm+ & Flacc+ & $\overline{\text { W56 }}$ & $\overline{\overline{\text { Dahm+ }}}$ & $\begin{array}{l}\text { Ct. Rate. } \\
{\left[10^{-4} s^{-1}\right]}\end{array}$ \\
\hline 481 & $6: 40: 42.5$ & $9: 41: 06.3$ & $\overline{c 1.00}$ & $\overline{-}$ & $\overline{-1}$ & $\overline{-}$ & 44619 & $\overline{-1}$ & 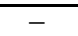 & $\overline{-}$ & $\overline{c<2.4}$ \\
\hline 482 & $6: 40: 42.6$ & $9: 42: 50.2$ & 1.00 & - & - & - & 4623 & - & - & - & $<3.1$ \\
\hline 483 & $6: 40: 42.7$ & $9: 29: 31.5$ & 1.00 & - & - & - & 4629 & - & - & - & $<1.5$ \\
\hline 484 & $6: 40: 42.7$ & $9: 42: 54.9$ & 1.00 & - & - & - & 4630 & - & - & - & $<3.1$ \\
\hline 485 & $6: 40: 42.7$ & 9:40:07.9 & 0.83 & - & $06404270+0940079$ & - & 4634 & $32 \dagger$ & - & - & $<2.1$ \\
\hline 486 & $6: 40: 42.7$ & $9: 27: 27.8$ & 1.00 & - & - & _- & 4633 & - & - & - & $<2.2$ \\
\hline $487 *$ & $6: 40: 42.7$ & $9: 27: 06.8$ & 0.50 & - & $06404273+0927067$ & 2830 & 4635 & - & - & - & $<2.3$ \\
\hline $488^{*}$ & $6: 40: 42.8$ & 9:33:35.1 & 0.50 & 57 & $06404278+0933350$ & - & 4638 & - & - & 112 & 2.6 \\
\hline 489 & $6: 40: 42.8$ & $9: 30: 36.6$ & 0.50 & - & $06404280+0930365$ & 2835 & 4639 & - & - & - & $<1.3$ \\
\hline $490 *$ & $6: 40: 42.8$ & $9: 39: 05.6$ & 0.54 & 58 & $06404280+0939056$ & - & - & - & - & - & 1.7 \\
\hline 491 & $6: 40: 42.8$ & 9:43:14.9 & 0.58 & - & $06404281+0943148$ & - & 4640 & - & - & - & $<3.3$ \\
\hline 492 & $6: 40: 42.8$ & $9: 29: 19.3$ & 1.00 & - & - & - & 4643 & - & - & - & $<1.6$ \\
\hline 493 & $6: 40: 42.9$ & $9: 38: 51.8$ & 0.50 & - & $06404294+0938517$ & - & - & - & - & - & $<1.6$ \\
\hline 494 & $6: 40: 43.1$ & 9:40:29.0 & 1.00 & - & - & - & 4650 & - & - & - & $<2.2$ \\
\hline 495 & $6: 40: 43.1$ & $9: 42: 34.5$ & 1.00 & - & - & - & 4652 & - & - & - & $<2.9$ \\
\hline 496 & $6: 40: 43.2$ & $9: 40: 23.6$ & 1.00 & - & - & - & 4654 & - & - & - & $<2.2$ \\
\hline 497 & $6: 40: 43.2$ & $9: 26: 49.4$ & 0.68 & - & $06404316+0926493$ & 2842 & 4653 & - & - & - & $<3.6$ \\
\hline 498 & $6: 40: 43.2$ & $9: 26: 52.5$ & 1.00 & - & - & - & 4656 & - & - & - & $<2.5$ \\
\hline 499 & $6: 40: 43.2$ & $9: 26: 57.1$ & 1.00 & - & - & - & 4657 & - & - & - & $<2.4$ \\
\hline 500 & $6: 40: 43.2$ & $9: 42: 42.6$ & 1.00 & - & - & - & 4659 & - & - & - & $<3.0$ \\
\hline 501 & $6: 40: 43.2$ & $9: 29: 47.3$ & 1.00 & - & - & - & 4660 & - & - & - & $<1.4$ \\
\hline 502 & $6: 40: 43.2$ & $9: 43: 44.8$ & 0.50 & - & $06404321+0943447$ & 2847 & 4662 & - & - & - & $<13.9$ \\
\hline $503 *$ & $6: 40: 43.2$ & $9: 31: 15.0$ & 0.50 & 59 & $06404323+0931149$ & 2845 & 4664 & - & - & 114 & 35.7 \\
\hline 504 & $6: 40: 43.3$ & 9:33:59.1 & 0.50 & - & $06404330+0933591$ & - & 4670 & - & - & - & $<0.9$ \\
\hline 505 & $6: 40: 43.3$ & $9: 43: 03.3$ & 0.50 & - & $06404330+0943033$ & 2849 & 4671 & - & - & - & $<3.2$ \\
\hline 506 & $6: 40: 43.4$ & $9: 43: 14.8$ & 1.00 & - & - & - & 4678 & - & - & - & $<3.3$ \\
\hline 507 & $6: 40: 43.4$ & 9:43:17.0 & 1.00 & - & - & - & 4679 & - & - & - & $<3.3$ \\
\hline $508 *$ & $6: 40: 43.4$ & $9: 30: 34.3$ & 0.50 & 60 & $06404340+0930343$ & - & 4680 & - & - & 116 & 4.0 \\
\hline $509 *$ & $6: 40: 43.4$ & $9: 28: 40.9$ & 0.50 & 61 & $06404342+0928409$ & 2853 & 4682 & - & - & 117 & 17.4 \\
\hline $510 *$ & $6: 40: 43.6$ & $9: 37: 20.5$ & 0.50 & 62 & $06404358+0937205$ & - & 4695 & - & - & - & 2.2 \\
\hline 511 & $6: 40: 43.6$ & $9: 40: 42.4$ & 0.50 & - & $06404360+0940423$ & - & 4696 & - & - & - & $<2.1$ \\
\hline 512 & $6: 40: 43.6$ & 9:33:39.9 & 1.00 & - & - & - & 4698 & - & - & - & $<0.9$ \\
\hline $513 *$ & $6: 40: 43.6$ & $9: 35: 11.5$ & 0.50 & 64 & $06404363+0935114$ & 2864 & 4700 & - & - & - & 3.1 \\
\hline $514 *$ & $6: 40: 43.6$ & 9:31:14.4 & 2.09 & 63 & - & - & - & - & - & - & 1.1 \\
\hline $515^{*}$ & $6: 40: 43.7$ & $9: 38: 35.7$ & 0.50 & 65 & $06404365+0938356$ & - & 4703 & - & - & 119 & 35.8 \\
\hline 516 & $6: 40: 43.7$ & 9:30:02.3 & 0.50 & - & $06404368+0930023$ & - & 4705 & - & - & - & $<1.3$ \\
\hline 517 & $6: 40: 43.7$ & $9: 43: 14.2$ & 1.00 & - & - & - & 4706 & - & - & - & $<3.3$ \\
\hline 518 & $6: 40: 43.7$ & $9: 28: 55.1$ & 1.00 & - & - & - & 4708 & - & - & - & $<3.5$ \\
\hline 519 & $6: 40: 43.8$ & $9: 39: 39.5$ & 0.50 & - & $06404381+0939395$ & - & - & - & - & - & $<1.7$ \\
\hline 520 & $6: 40: 43.8$ & $9: 38: 49.2$ & 1.00 & - & - & - & 4719 & - & - & - & $<1.5$ \\
\hline $521 *$ & $6: 40: 43.9$ & $9: 28: 55.2$ & 0.50 & 66 & $06404386+0928552$ & 2868 & 4722 & - & - & - & 3.9 \\
\hline 522 & $6: 40: 43.9$ & $9: 42: 35.1$ & 1.00 & - & - & - & 4725 & - & - & - & $<2.9$ \\
\hline 523 & $6: 40: 44.0$ & 9:28:22.1 & 1.00 & - & - & - & 4734 & - & - & - & $<1.9$ \\
\hline 524 & $6: 40: 44.0$ & 9:43:39.6 & 1.00 & - & - & - & 4735 & - & - & - & $<6.7$ \\
\hline 525 & $6: 40: 44.0$ & $9: 29: 21.8$ & 1.00 & - & - & - & 4741 & - & - & - & $<1.5$ \\
\hline 526 & $6: 40: 44.0$ & $9: 29: 32.1$ & 1.00 & - & - & - & 4743 & - & - & - & $<1.4$ \\
\hline 527 & $6: 40: 44.1$ & $9: 28: 05.9$ & 0.50 & - & $06404411+0928058$ & - & 4748 & - & - & - & $<1.8$ \\
\hline 528 & $6: 40: 44.2$ & 9:41:04.0 & 1.00 & - & - & - & 4752 & - & - & - & $<2.1$ \\
\hline 529 & $6: 40: 44.2$ & 9:43:05.7 & 0.50 & - & $06404418+0943056$ & 2877 & 4754 & - & - & - & $<3.2$ \\
\hline $530 *$ & $6: 40: 44.2$ & $9: 36: 35.9$ & 1.05 & 67 & - & - & - & - & - & - & 1.5 \\
\hline 531 & $6: 40: 44.4$ & $9: 42: 25.8$ & 0.50 & - & $06404440+0942258$ & 2887 & 4764 & - & - & - & $<2.9$ \\
\hline 532 & $6: 40: 44.5$ & $9: 40: 22.9$ & 1.00 & - & - & - & 4770 & - & - & - & $<1.9$ \\
\hline 533 & $6: 40: 44.5$ & $9: 29: 43.2$ & 0.50 & - & $06404452+0929432$ & 2891 & 4772 & - & - & - & $<1.3$ \\
\hline $534^{*}$ & $6: 40: 44.6$ & $9: 32: 26.2$ & 0.50 & 68 & $06404459+0932261$ & 2895 & 4776 & 36 & - & 123 & 6.8 \\
\hline $535^{*}$ & $6: 40: 44.6$ & $9: 27: 44.0$ & 0.50 & - & $06404459+0927439$ & 2897 & 4775 & - & - & - & $<1.9$ \\
\hline 536 & $6: 40: 44.7$ & $9: 28: 04.5$ & 1.00 & - & - & - & 4785 & - & - & - & $<1.8$ \\
\hline 537 & $6: 40: 44.7$ & $9: 30: 45.9$ & 1.00 & - & - & - & 4786 & - & - & - & $<1.2$ \\
\hline 538 & $6: 40: 44.7$ & 9:30:00.8 & 0.50 & - & $06404471+0930008$ & 2899 & 4788 & - & - & - & $<1.2$ \\
\hline $539 *$ & $6: 40: 44.8$ & 9:34:04.6 & 1.00 & 69 & - & - & - & - & - & - & 1.1 \\
\hline $540 *$ & $6: 40: 44.9$ & $9: 38: 49.8$ & 0.50 & 70 & $06404494+0938497$ & 2906 & 4798 & - & - & - & 2.4 \\
\hline
\end{tabular}


Table 3. (Continued)

\begin{tabular}{|c|c|c|c|c|c|c|c|c|c|c|c|}
\hline$\overline{\mathrm{N}}$ & $\begin{array}{c}\mathrm{RA} \\
{[\mathrm{h} \mathrm{m} \mathrm{s}]}\end{array}$ & $\begin{array}{c}\text { Dec. } \\
{[\mathrm{d} \mathrm{m} \mathrm{s}]}\end{array}$ & $\begin{array}{c}\text { Id. rad. } \\
\text { ["] }\end{array}$ & $\overline{\mathrm{ACIS}}$ & 2Mass & Reb.+ & $\overline{\mathrm{Lamm+}}$ & Flacc+ & $\overline{\text { W56 }}$ & $\overline{\overline{\text { Dahm+ }}}$ & $\begin{array}{l}\text { Ct. Rate. } \\
{\left[10^{-4} s^{-1}\right]}\end{array}$ \\
\hline 541 & $\overline{6: 40: 45.0}$ & $9: 30: 01.2$ & $\overline{0.50}$ & $\overline{-}$ & $06404500+0930011$ & $=$ & $\overline{48803}$ & $=$ & 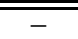 & $\overline{-1}$ & $\overline{c<1.2}$ \\
\hline 542 & $6: 40: 45.0$ & $9: 33: 22.9$ & 0.50 & - & $06404502+0933229$ & 2909 & 4806 & - & - & - & $<0.8$ \\
\hline 543 & $6: 40: 45.0$ & $9: 27: 43.3$ & 1.00 & - & - & - & 4805 & - & - & - & $<1.9$ \\
\hline 544 & $6: 40: 45.1$ & $9: 42: 06.5$ & 1.00 & - & - & - & 4813 & - & - & - & $<2.8$ \\
\hline 545 & $6: 40: 45.1$ & 9:43:01.7 & 0.64 & - & $06404510+0943016$ & - & 4814 & - & - & - & $<3.3$ \\
\hline 546 & $6: 40: 45.1$ & $9: 41: 28.7$ & 0.50 & - & $06404510+0941287$ & 2912 & 4816 & - & - & - & $<2.2$ \\
\hline 547 & $6: 40: 45.1$ & $9: 28: 31.6$ & 0.50 & - & $06404513+0928316$ & 2914 & 4821 & - & - & - & $<1.8$ \\
\hline 548 & $6: 40: 45.2$ & $9: 43: 11.5$ & 1.00 & - & - & - & 4824 & - & - & - & $<3.4$ \\
\hline $549 *$ & $6: 40: 45.2$ & $9: 28: 44.4$ & 0.50 & 71 & $06404516+0928444$ & 2915 & 4826 & - & - & 130 & 34.7 \\
\hline 550 & $6: 40: 45.2$ & 9:43:17.1 & 0.50 & - & $06404519+0943170$ & 2917 & 4827 & - & - & - & $<3.4$ \\
\hline 551 & $6: 40: 45.3$ & 9:43:03.4 & 1.00 & - & - & - & 4832 & - & - & - & $<3.3$ \\
\hline 552 & $6: 40: 45.3$ & $9: 35: 45.0$ & 0.50 & - & $06404526+0935450$ & - & - & - & - & - & $<0.8$ \\
\hline 553 & $6: 40: 45.3$ & $9: 42: 35.1$ & 1.00 & - & - & - & 4836 & - & - & - & $<3.0$ \\
\hline 554 & $6: 40: 45.3$ & $9: 38: 20.0$ & 0.95 & - & $06404530+0938199$ & - & - & - & - & - & $<1.3$ \\
\hline 555 & $6: 40: 45.3$ & $9: 38: 47.6$ & 0.50 & - & $06404534+0938475$ & - & 4838 & - & - & - & $<1.6$ \\
\hline 556 & $6: 40: 45.4$ & $9: 37: 51.5$ & 0.50 & - & $06404539+0937515$ & 2922 & 4841 & - & - & - & $<1.2$ \\
\hline 557 & $6: 40: 45.4$ & 9:40:29.7 & 0.50 & - & $06404540+0940297$ & 2923 & 4843 & - & - & - & $<1.9$ \\
\hline 558 & $6: 40: 45.4$ & 9:43:17.9 & 1.00 & - & - & - & 4844 & - & - & - & $<3.4$ \\
\hline 559 & $6: 40: 45.5$ & $9: 26: 56.0$ & 1.50 & - & - & 2925 & - & - & - & - & $<2.2$ \\
\hline 560 & $6: 40: 45.5$ & $9: 28: 00.5$ & 0.50 & - & $06404552+0928005$ & - & - & - & - & - & $<1.7$ \\
\hline 561 & $6: 40: 45.6$ & $9: 28: 40.0$ & 1.00 & - & - & - & 4858 & - & - & - & $<1.7$ \\
\hline 562 & $6: 40: 45.6$ & $9: 28: 21.3$ & 1.00 & - & - & - & 4861 & - & - & - & $<1.8$ \\
\hline 563 & $6: 40: 45.8$ & $9: 28: 02.6$ & 0.50 & - & $06404576+0928025$ & 2934 & 4869 & - & - & - & $<1.7$ \\
\hline $564 *$ & $6: 40: 45.9$ & $9: 38: 44.4$ & 0.50 & 72 & $06404587+0938443$ & 2938 & 4875 & - & - & 131 & 1.8 \\
\hline 565 & $6: 40: 45.9$ & $9: 39: 19.5$ & 0.69 & - & $06404590+0939195$ & - & 4877 & - & - & - & $<1.7$ \\
\hline $566^{*}$ & $6: 40: 45.9$ & $9: 26: 44.2$ & 5.27 & 73 & - & - & - & - & - & - & 1.8 \\
\hline 567 & $6: 40: 45.9$ & $9: 36: 37.8$ & 1.00 & - & - & 2940 & 4878 & - & - & - & $<0.9$ \\
\hline 568 & $6: 40: 46.0$ & $9: 30: 51.8$ & 1.00 & - & - & - & 4882 & - & - & - & $<1.0$ \\
\hline 569 & $6: 40: 46.1$ & $9: 43: 15.5$ & 1.00 & - & - & - & 4888 & - & - & - & $<3.4$ \\
\hline 570 & $6: 40: 46.1$ & 9:39:46.4 & 0.67 & - & $06404614+0939464$ & - & 4890 & - & - & - & $<1.8$ \\
\hline 571 & $6: 40: 46.2$ & $9: 42: 46.9$ & 1.00 & - & - & - & 4893 & - & - & - & $<3.2$ \\
\hline 572 & $6: 40: 46.2$ & $9: 35: 12.4$ & 1.00 & - & - & - & 4895 & - & - & - & $<1.7$ \\
\hline 573 & $6: 40: 46.3$ & $9: 42: 51.5$ & 1.00 & - & - & - & 4898 & - & - & - & $<3.2$ \\
\hline 574 & $6: 40: 46.3$ & $9: 43: 06.3$ & 1.00 & - & - & - & 4901 & - & - & - & $<3.3$ \\
\hline 575 & $6: 40: 46.4$ & $9: 42: 26.5$ & 1.00 & - & - & - & 4903 & - & - & - & $<2.8$ \\
\hline 576 & $6: 40: 46.4$ & $9: 27: 53.7$ & 1.00 & - & - & - & 4906 & - & - & - & $<1.8$ \\
\hline 577 & $6: 40: 46.4$ & $9: 41: 56.8$ & 0.50 & - & $06404644+0941568$ & 2947 & 4908 & - & - & - & $<2.5$ \\
\hline 578 & $6: 40: 46.5$ & $9: 42: 04.5$ & 1.00 & - & - & - & 4909 & - & - & - & $<2.6$ \\
\hline 579 & $6: 40: 46.6$ & $9: 33: 52.4$ & 0.50 & - & $06404655+0933523$ & 2950 & 4913 & - & - & - & $<0.8$ \\
\hline $580^{*}$ & $6: 40: 46.6$ & $9: 42: 49.7$ & 0.61 & - & $06404658+0942497$ & - & 4914 & - & - & 135 & $<3.2$ \\
\hline 581 & $6: 40: 46.6$ & $9: 41: 54.8$ & 0.50 & - & $06404663+0941547$ & - & 4919 & - & - & - & $<2.5$ \\
\hline 582 & $6: 40: 46.7$ & $9: 42: 00.8$ & 1.00 & - & - & - & 4921 & - & - & - & $<2.6$ \\
\hline 583 & $6: 40: 46.7$ & $9: 37: 06.3$ & 0.50 & - & $06404667+0937062$ & - & 4922 & - & - & - & $<1.0$ \\
\hline $584 *$ & $6: 40: 46.7$ & 9:40:01.4 & 0.50 & - & $06404668+0940014$ & 2957 & 4923 & - & - & 137 & $<1.7$ \\
\hline $585^{*}$ & $6: 40: 46.7$ & $9: 40: 22.3$ & 2.69 & 74 & - & - & - & - & - & - & 5.4 \\
\hline $586^{*}$ & $6: 40: 46.8$ & $9: 32: 57.5$ & 0.50 & 76 & $06404677+0932575$ & - & 4928 & - & - & - & 1.8 \\
\hline 587 & $6: 40: 46.8$ & $9: 42: 56.9$ & 0.61 & - & $06404677+0942568$ & - & 4927 & - & - & - & $<3.2$ \\
\hline 588 & $6: 40: 46.8$ & $9: 28: 44.5$ & 1.00 & - & - & - & 4929 & - & - & - & $<1.6$ \\
\hline 589 & $6: 40: 46.8$ & $9: 26: 53.9$ & 1.00 & - & - & - & 4935 & - & - & - & $<2.5$ \\
\hline $590 *$ & $6: 40: 46.9$ & $9: 32: 30.9$ & 0.50 & 75 & $06404686+0932309$ & 2969 & 4937 & 40 & - & 139 & 10.4 \\
\hline 591 & $6: 40: 46.9$ & $9: 26: 39.1$ & 1.00 & - & - & - & 4938 & - & - & - & $<4.9$ \\
\hline 592 & $6: 40: 46.9$ & $9: 33: 22.3$ & 1.00 & - & - & - & 4939 & - & - & - & $<0.8$ \\
\hline 593 & $6: 40: 47.0$ & $9: 38: 37.4$ & 0.50 & - & $06404696+0938374$ & - & 4943 & - & - & - & $<1.3$ \\
\hline $594 *$ & $6: 40: 47.1$ & 9:30:19.7 & 0.50 & 77 & $06404710+0930197$ & - & 4954 & - & - & - & 1.0 \\
\hline $595^{*}$ & $6: 40: 47.1$ & $9: 32: 40.1$ & 0.50 & 78 & $06404711+0932401$ & 2974 & 4955 & 41 & 95 & 142 & 61.6 \\
\hline $596^{*}$ & $6: 40: 47.1$ & $9: 42: 07.7$ & 0.50 & 79 & $06404712+0942077$ & 2975 & 4956 & - & - & 143 & 2.7 \\
\hline 597 & $6: 40: 47.1$ & $9: 28: 55.9$ & 0.50 & - & $06404712+0928558$ & - & 4958 & - & - & - & $<1.5$ \\
\hline $598 *$ & $6: 40: 47.2$ & $9: 28: 49.9$ & 0.50 & - & $06404717+0928498$ & 2979 & 4960 & - & - & - & $<1.6$ \\
\hline 599 & $6: 40: 47.2$ & $9: 41: 55.9$ & 1.00 & - & - & - & 4961 & - & - & - & $<2.7$ \\
\hline $600^{*}$ & $6: 40: 47.2$ & 9:31:08.7 & 0.50 & 80 & $06404722+0931086$ & 2981 & 4967 & - & - & 145 & 1.5 \\
\hline
\end{tabular}


Table 3. (Continued)

\begin{tabular}{|c|c|c|c|c|c|c|c|c|c|c|c|}
\hline$\overline{\mathrm{N}}$ & $\begin{array}{c}\mathrm{RA} \\
{[\mathrm{h} \mathrm{m} \mathrm{s}]}\end{array}$ & $\begin{array}{c}\text { Dec. } \\
{[\mathrm{d} \mathrm{m} \mathrm{s}]}\end{array}$ & $\begin{array}{c}\text { Id. rad. } \\
\text { ["] } \\
\end{array}$ & ACIS & 2Mass & Reb.+ & $\overline{\text { Lamm+ }}$ & Flacc+ & $\overline{\text { W56 }}$ & $\overline{\overline{\text { Dahm+ }}}$ & $\begin{array}{l}\text { Ct. Rate. } \\
{\left[10^{-4} s^{-1}\right]}\end{array}$ \\
\hline$\overline{6001}$ & $6: 40: 47.2$ & $9: 41: 47.5$ & $\overline{1.00}$ & $\overline{-}$ & $\overline{-1}$ & $\overline{-1}$ & 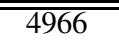 & - & - & - & $<<2.6$ \\
\hline 602 & $6: 40: 47.3$ & 9:29:01.8 & 1.00 & - & - & - & 4968 & - & - & - & $<1.5$ \\
\hline 603 & $6: 40: 47.3$ & $9: 42: 41.8$ & 1.00 & - & - & - & 4969 & - & - & - & $<3.2$ \\
\hline 604 & $6: 40: 47.3$ & $9: 42: 26.8$ & 0.50 & - & $06404730+0942268$ & - & 4970 & - & - & - & $<3.0$ \\
\hline 605 & $6: 40: 47.3$ & $9: 27: 29.2$ & 1.00 & - & - & - & 4973 & - & - & - & $<2.1$ \\
\hline 606 & $6: 40: 47.4$ & $9: 42: 42.3$ & 0.59 & - & $06404737+0942423$ & - & 4978 & - & - & - & $<3.3$ \\
\hline 607 & $6: 40: 47.5$ & 9:32:48.6 & 0.50 & - & $06404750+0932485$ & - & 4985 & - & - & - & $<0.8$ \\
\hline 608 & $6: 40: 47.5$ & $9: 42: 53.5$ & 0.86 & - & $06404752+0942534$ & - & 4994 & - & - & - & $<3.3$ \\
\hline $609 *$ & $6: 40: 47.6$ & $9: 40: 33.1$ & 0.50 & 81 & $06404756+0940331$ & 2988 & 4991 & - & - & - & 1.9 \\
\hline 610 & $6: 40: 47.7$ & $9: 41: 38.1$ & 0.62 & - & 06404769+0941381 & - & 4998 & - & - & - & $<2.5$ \\
\hline 611 & $6: 40: 47.7$ & $9: 43: 26.5$ & 1.00 & - & - & - & 4999 & - & - & - & $<4.6$ \\
\hline 612 & $6: 40: 47.7$ & $9: 42: 10.7$ & 0.50 & - & $06404771+0942106$ & 2992 & 5002 & - & - & - & $<2.9$ \\
\hline 613 & $6: 40: 47.7$ & $9: 29: 42.1$ & 0.55 & - & $06404771+0929421$ & 2994 & 5001 & - & - & - & $<1.2$ \\
\hline 614 & $6: 40: 47.8$ & $9: 26: 37.4$ & 1.00 & - & - & - & 5004 & - & - & - & $<4.2$ \\
\hline $615^{*}$ & $6: 40: 47.8$ & $9: 26: 45.2$ & 2.62 & 82 & - & - & - & - & - & - & 6.2 \\
\hline 616 & $6: 40: 47.9$ & $9: 27: 47.4$ & 1.00 & - & - & - & 5011 & - & - & - & $<1.7$ \\
\hline 617 & $6: 40: 47.9$ & $9: 29: 44.9$ & 1.00 & - & - & - & 5012 & - & - & - & $<1.3$ \\
\hline $618^{*}$ & $6: 40: 47.9$ & 9:33:03.1 & 0.50 & 83 & $06404792+0933031$ & 2997 & 5015 & - & - & - & 10.5 \\
\hline 619 & $6: 40: 48.0$ & $9: 27: 29.5$ & 0.50 & - & $06404796+0927294$ & 2999 & 5018 & - & - & - & $<2.0$ \\
\hline $620 *$ & 6:40:48.0 & 9:32:03.7 & 0.50 & 84 & $06404799+0932037$ & 3000 & 5019 & - & - & - & 0.8 \\
\hline 621 & $6: 40: 48.0$ & $9: 31: 20.9$ & 0.50 & - & $06404801+0931208$ & 3002 & 5023 & - & - & - & $<0.8$ \\
\hline $622 *$ & $6: 40: 48.1$ & $9: 34: 05.5$ & 0.50 & 85 & $06404807+0934054$ & 3003 & 5027 & - & - & - & 0.8 \\
\hline 623 & $6: 40: 48.1$ & $9: 27: 31.2$ & 1.00 & - & - & - & 5026 & - & - & - & $<1.9$ \\
\hline 624 & $6: 40: 48.2$ & $9: 26: 54.1$ & 1.00 & - & - & - & 5032 & - & - & - & $<2.8$ \\
\hline $625 *$ & $6: 40: 48.2$ & $9: 27: 00.8$ & 0.50 & 86 & $06404819+0927008$ & 3010 & 5035 & - & - & 151 & 3.9 \\
\hline 626 & $6: 40: 48.2$ & $9: 27: 42.6$ & 1.00 & - & - & - & 5036 & - & - & - & $<1.8$ \\
\hline 627 & $6: 40: 48.2$ & $9: 36: 23.5$ & 1.00 & - & - & - & 5037 & - & - & - & $<0.9$ \\
\hline 628 & $6: 40: 48.3$ & $9: 30: 54.6$ & 0.50 & - & $06404825+0930546$ & - & 5040 & - & - & - & $<0.9$ \\
\hline $629 *$ & $6: 40: 48.3$ & $9: 36: 38.7$ & 0.50 & 87 & $06404827+0936386$ & 3013 & 5041 & 44 & 97 & 152 & 60.0 \\
\hline 630 & $6: 40: 48.4$ & $9: 42: 34.4$ & 1.00 & - & - & - & 5050 & - & - & - & $<3.2$ \\
\hline $631 *$ & $6: 40: 48.5$ & $9: 34: 16.8$ & 0.50 & 88 & $06404853+0934167$ & 3016 & 5055 & - & - & - & 1.5 \\
\hline 632 & $6: 40: 48.6$ & $9: 36: 48.1$ & 0.50 & - & $06404861+0936481$ & - & - & - & - & - & $<0.9$ \\
\hline $633^{*}$ & $6: 40: 48.6$ & $9: 32: 52.4$ & 0.50 & 90 & $06404862+0932524$ & 3018 & 5063 & 45 & 101 & 155 & 25.6 \\
\hline $634^{*}$ & 6:40:48.6 & $9: 35: 57.8$ & 0.50 & 89 & $06404862+0935578$ & 3017 & 5061 & - & - & 154 & 15.7 \\
\hline 635 & 6:40:48.6 & $9: 26: 55.6$ & 1.00 & - & - & - & 5062 & - & - & - & $<2.4$ \\
\hline 636 & $6: 40: 48.6$ & $9: 32: 44.1$ & 1.00 & - & - & - & 5065 & - & - & - & $<0.8$ \\
\hline 637 & $6: 40: 48.7$ & 9:39:01.8 & 0.63 & - & $06404873+0939017$ & - & - & - & - & - & $<1.3$ \\
\hline $638^{*}$ & $6: 40: 48.8$ & $9: 32: 42.6$ & 0.50 & 91 & $06404875+0932425$ & 3019 & 5070 & - & - & 156 & 18.3 \\
\hline 639 & $6: 40: 48.8$ & 9:42:01.8 & 0.61 & - & $06404882+0942017$ & - & 5072 & - & - & - & $<2.7$ \\
\hline 640 & $6: 40: 48.8$ & $9: 34: 05.8$ & 1.00 & - & - & - & 5073 & - & - & - & $<0.7$ \\
\hline $641 *$ & $6: 40: 48.8$ & $9: 43: 25.7$ & 0.50 & - & $06404884+0943256$ & 3023 & 5074 & - & - & 157 & $<12.8$ \\
\hline 642 & 6:40:48.9 & $9: 31: 10.3$ & 0.82 & - & $06404887+0931103$ & - & 5081 & - & - & - & $<0.9$ \\
\hline 643 & $6: 40: 48.9$ & $9: 29: 16.4$ & 1.00 & - & - & - & 5077 & - & - & - & $<1.3$ \\
\hline 644 & $6: 40: 48.9$ & $9: 41: 36.3$ & 1.00 & - & - & - & 5083 & - & - & - & $<2.3$ \\
\hline 645 & $6: 40: 48.9$ & 9:30:03.0 & 1.00 & - & - & - & 5085 & - & - & - & $<1.2$ \\
\hline 646 & $6: 40: 49.0$ & $9: 42: 39.2$ & 0.50 & - & $06404896+0942391$ & 3026 & 5089 & - & - & - & $<3.0$ \\
\hline $647 *$ & $6: 40: 49.0$ & $9: 36: 55.2$ & 1.99 & 92 & - & - & - & - & - & - & 0.9 \\
\hline 648 & $6: 40: 49.0$ & $9: 29: 14.3$ & 1.00 & - & - & - & 5096 & - & - & - & $<1.3$ \\
\hline $649 *$ & $6: 40: 49.1$ & $9: 37: 36.3$ & 0.50 & 93 & $06404912+0937363$ & - & 5099 & - & - & 158 & 2.8 \\
\hline 650 & $6: 40: 49.2$ & $9: 37: 14.5$ & 0.50 & - & $06404916+0937145$ & - & 5101 & - & - & - & $<0.9$ \\
\hline 651 & $6: 40: 49.2$ & $9: 30: 51.3$ & 1.00 & - & - & - & 5102 & - & - & - & $<1.0$ \\
\hline 652 & $6: 40: 49.2$ & $9: 27: 11.3$ & 0.50 & - & $06404921+0927112$ & - & 5104 & - & - & - & $<2.1$ \\
\hline 653 & $6: 40: 49.3$ & $9: 33: 43.8$ & 0.50 & - & $06404933+0933437$ & - & - & - & - & - & $<0.7$ \\
\hline 654 & $6: 40: 49.3$ & $9: 31: 57.7$ & 1.00 & - & - & - & 5112 & - & - & - & $<0.8$ \\
\hline 655 & $6: 40: 49.4$ & $9: 30: 54.7$ & 1.00 & - & - & - & 5113 & - & - & - & $<0.9$ \\
\hline 656 & $6: 40: 49.4$ & $9: 36: 57.1$ & 0.50 & - & $06404939+0936571$ & - & - & - & - & - & $<0.9$ \\
\hline $657 *$ & $6: 40: 49.5$ & $9: 31: 41.6$ & 1.55 & 94 & - & - & - & - & - & - & 2.3 \\
\hline 658 & $6: 40: 49.5$ & 9:30:32.7 & 1.00 & - & - & - & 5119 & - & - & - & $<1.1$ \\
\hline 659 & $6: 40: 49.6$ & $9: 38: 40.4$ & 0.50 & - & $06404955+0938403$ & 3043 & 5120 & - & - & - & $<1.2$ \\
\hline 660 & $6: 40: 49.6$ & $9: 38: 39.7$ & 1.00 & - & - & - & 5124 & - & - & - & $<1.2$ \\
\hline
\end{tabular}


Table 3. (Continued)

\begin{tabular}{|c|c|c|c|c|c|c|c|c|c|c|c|}
\hline$\overline{\mathrm{N}}$ & $\begin{array}{c}\mathrm{RA} \\
{[\mathrm{h} \mathrm{m} \mathrm{s}]}\end{array}$ & $\begin{array}{c}\text { Dec. } \\
{[\mathrm{d} \mathrm{m} \mathrm{s}]}\end{array}$ & $\begin{array}{c}\text { Id. rad. } \\
\text { ["] }\end{array}$ & $\overline{\mathrm{ACIS}}$ & 2Mass & Reb.+ & $\overline{\mathrm{Lamm+}}$ & Flacc+ & W56 & $\overline{\overline{\text { Dahm+ }}}$ & $\begin{array}{l}\text { Ct. Rate. } \\
{\left[10^{-4} s^{-1}\right]}\end{array}$ \\
\hline 6661 & $6: 40: 49.7$ & $9: 41: 07.3$ & 1.00 & $\overline{-}$ & $\overline{-1}$ & $=$ & $\overline{5133}$ & $=$ & $\overline{-1}$ & $\overline{-1}$ & $\overline{c<2.0}$ \\
\hline 662 & $6: 40: 49.9$ & $9: 41: 49.2$ & 0.50 & - & $06404986+0941491$ & - & 5139 & - & - & - & $<2.5$ \\
\hline $663^{*}$ & $6: 40: 49.9$ & $9: 36: 49.5$ & 0.50 & 95 & $06404989+0936494$ & 3051 & 5143 & - & 105 & 167 & 11.9 \\
\hline 664 & $6: 40: 50.0$ & $9: 42: 15.2$ & 1.00 & - & - & - & 5147 & - & - & - & $<2.8$ \\
\hline 665 & $6: 40: 50.0$ & $9: 27: 27.9$ & 1.00 & - & - & - & 5151 & - & - & - & $<1.8$ \\
\hline 666 & $6: 40: 50.1$ & 9:37:10.7 & 1.00 & - & - & - & 5154 & - & - & - & $<0.9$ \\
\hline 667 & $6: 40: 50.1$ & $9: 35: 20.8$ & 1.00 & - & - & - & 5156 & - & - & - & $<0.7$ \\
\hline $668^{*}$ & $6: 40: 50.1$ & $9: 29: 32.5$ & 3.27 & 96 & - & - & - & - & - & - & 1.8 \\
\hline $669^{*}$ & $6: 40: 50.1$ & $9: 30: 39.9$ & 0.50 & 97 & $06405010+0930399$ & 3059 & 5160 & - & - & 171 & 14.1 \\
\hline 670 & $6: 40: 50.1$ & 9:43:00.3 & 1.00 & - & - & - & 5162 & - & - & - & $<3.3$ \\
\hline 671 & $6: 40: 50.2$ & $9: 37: 25.5$ & 0.50 & - & $06405018+0937255$ & 3062 & 5166 & - & 106 & - & $<0.9$ \\
\hline 672 & $6: 40: 50.2$ & 9:33:49.1 & 0.54 & - & $06405018+0933490$ & 3063 & 5167 & - & - & - & $<0.7$ \\
\hline 673 & $6: 40: 50.2$ & $9: 30: 23.0$ & 0.50 & - & $06405023+0930229$ & - & 5170 & - & - & - & $<1.0$ \\
\hline 674 & $6: 40: 50.3$ & $9: 29: 36.6$ & 0.50 & - & $06405025+0929365$ & - & 5171 & - & - & - & $<1.8$ \\
\hline 675 & $6: 40: 50.3$ & $9: 32: 24.2$ & 0.50 & - & $06405026+0932241$ & - & 5172 & - & - & - & $<0.8$ \\
\hline 676 & $6: 40: 50.3$ & $9: 27: 32.3$ & 0.66 & - & $06405029+0927323$ & 3070 & 5175 & - & - & - & $<1.7$ \\
\hline 677 & $6: 40: 50.3$ & 9:43:11.8 & 1.00 & - & - & - & 5178 & - & - & - & $<3.7$ \\
\hline 678 & $6: 40: 50.4$ & $9: 35: 35.9$ & 0.50 & - & $06405036+0935359$ & - & - & - & - & - & $<0.7$ \\
\hline 679 & $6: 40: 50.4$ & $9: 36: 47.2$ & 0.61 & - & $06405037+0936471$ & - & 5183 & - & - & - & $<0.8$ \\
\hline 680 & $6: 40: 50.4$ & $9: 27: 58.5$ & 1.00 & - & - & - & 5182 & - & - & - & $<1.5$ \\
\hline $681 *$ & $6: 40: 50.5$ & $9: 32: 19.7$ & 1.00 & 98 & - & - & - & - & - & - & 10.2 \\
\hline 682 & $6: 40: 50.5$ & $9: 41: 50.7$ & 1.00 & - & - & - & 5191 & - & - & - & $<2.4$ \\
\hline 683 & $6: 40: 50.6$ & $9: 42: 55.0$ & 0.52 & - & $06405060+0942549$ & 3080 & 5194 & - & - & - & $<3.2$ \\
\hline $684 *$ & $6: 40: 50.6$ & $9: 31: 31.5$ & 0.50 & 99 & $06405063+0931314$ & 3081 & 5199 & - & - & - & 1.2 \\
\hline 685 & $6: 40: 50.6$ & $9: 39: 27.8$ & 1.00 & - & - & - & 5200 & - & - & - & $<1.3$ \\
\hline 686 & $6: 40: 50.7$ & $9: 42: 36.4$ & 0.58 & - & $06405069+0942364$ & - & 5201 & - & - & - & $<2.7$ \\
\hline 687 & $6: 40: 50.8$ & $9: 27: 26.1$ & 0.80 & - & $06405076+0927260$ & - & 5206 & - & - & - & $<1.8$ \\
\hline 688 & $6: 40: 50.8$ & $9: 29: 21.1$ & 1.00 & - & - & - & 5207 & - & - & - & $<1.3$ \\
\hline $689 *$ & $6: 40: 50.8$ & $9: 41: 55.3$ & 0.50 & 100 & $06405078+0941553$ & 3087 & 5210 & - & - & 180 & 3.7 \\
\hline 690 & $6: 40: 50.8$ & 9:40:09.2 & 1.00 & - & - & - & 5211 & - & - & - & $<1.6$ \\
\hline 691 & $6: 40: 50.8$ & $9: 36: 42.4$ & 0.50 & - & $06405082+0936424$ & - & - & - & - & - & $<0.8$ \\
\hline 692 & $6: 40: 50.9$ & $9: 27: 19.5$ & 1.00 & - & - & - & 5214 & - & - & - & $<1.8$ \\
\hline 693 & $6: 40: 50.9$ & $9: 43: 12.6$ & 1.00 & - & - & - & 5215 & - & - & - & $<3.6$ \\
\hline 694 & $6: 40: 50.9$ & 9:41:09.4 & 0.77 & - & $06405091+0941093$ & - & 5220 & - & - & - & $<1.9$ \\
\hline 695 & $6: 40: 51.0$ & $9: 27: 05.2$ & 0.50 & - & $06405097+0927052$ & 3093 & 5221 & - & - & - & $<1.9$ \\
\hline 696 & $6: 40: 51.0$ & $9: 31: 32.1$ & 0.50 & - & $06405101+0931320$ & - & 5224 & - & - & - & $<0.8$ \\
\hline 697 & $6: 40: 51.0$ & $9: 37: 14.8$ & 0.50 & - & $06405103+0937147$ & - & 5226 & - & - & - & $<0.8$ \\
\hline $698^{*}$ & $6: 40: 51.0$ & $9: 38: 52.9$ & 1.49 & 101 & - & - & - & - & - & - & 3.3 \\
\hline 699 & $6: 40: 51.1$ & $9: 31: 22.7$ & 1.00 & - & - & - & 5231 & - & - & - & $<0.8$ \\
\hline 700 & $6: 40: 51.1$ & $9: 39: 43.1$ & 0.50 & - & $06405111+0939431$ & - & 5232 & - & - & - & $<1.4$ \\
\hline 701 & $6: 40: 51.2$ & $9: 26: 25.3$ & 0.67 & - & $06405116+0926252$ & - & 5234 & - & - & - & $<2.9$ \\
\hline 702 & $6: 40: 51.2$ & $9: 42: 17.8$ & 1.00 & - & - & - & 5237 & - & - & - & $<2.6$ \\
\hline 703 & $6: 40: 51.2$ & $9: 27: 57.8$ & 0.50 & - & $06405121+0927578$ & 3099 & 5238 & - & - & - & $<1.5$ \\
\hline 704 & $6: 40: 51.2$ & $9: 31: 33.4$ & 1.00 & - & - & - & 5242 & - & - & - & $<0.8$ \\
\hline $705^{*}$ & $6: 40: 51.3$ & $9: 36: 39.0$ & 0.50 & - & $06405130+0936390$ & 3101 & 5244 & - & - & - & $<0.7$ \\
\hline 706 & $6: 40: 51.3$ & $9: 26: 26.9$ & 1.00 & - & - & - & 5243 & - & - & - & $<2.4$ \\
\hline 707 & $6: 40: 51.3$ & 9:30:20.7 & 0.50 & - & $06405133+0930207$ & - & - & - & - & - & $<1.0$ \\
\hline 708 & $6: 40: 51.3$ & $9: 27: 06.7$ & 1.00 & - & - & - & 5248 & - & - & - & $<1.8$ \\
\hline 709 & $6: 40: 51.4$ & $9: 27: 45.0$ & 0.53 & - & $06405136+0927449$ & - & 5249 & - & - & - & $<1.6$ \\
\hline $710 *$ & $6: 40: 51.4$ & $9: 30: 13.4$ & 0.50 & 103 & $06405137+0930133$ & 3103 & 5252 & - & - & 187 & 2.5 \\
\hline 711 & $6: 40: 51.4$ & $9: 41: 21.4$ & 0.50 & - & $06405142+0941213$ & 3106 & 5257 & - & - & - & $<1.9$ \\
\hline $712 *$ & $6: 40: 51.5$ & $9: 37: 14.5$ & 0.50 & 104 & $06405146+0937144$ & 3107 & 5263 & - & 111 & - & 8.6 \\
\hline 713 & $6: 40: 51.5$ & $9: 31: 46.8$ & 0.50 & - & $06405146+0931468$ & 3108 & 5262 & - & - & - & $<0.8$ \\
\hline 714 & $6: 40: 51.5$ & $9: 26: 42.7$ & 1.00 & - & - & - & 5266 & - & - & - & $<2.1$ \\
\hline $715^{*}$ & $6: 40: 51.5$ & $9: 43: 24.2$ & 0.50 & 102 & $06405154+0943242$ & 3111 & 5268 & - & - & 188 & 3.6 \\
\hline $716^{*}$ & $6: 40: 51.6$ & $9: 28: 44.5$ & 0.50 & 105 & $06405159+0928445$ & 3112 & 5274 & 50 & - & 189 & 28.8 \\
\hline 717 & $6: 40: 51.6$ & $9: 26: 58.2$ & 0.50 & - & $06405160+0926581$ & - & 5275 & - & - & - & $<1.9$ \\
\hline 718 & $6: 40: 51.6$ & $9: 26: 42.2$ & 0.50 & - & $06405164+0926421$ & 3115 & 5277 & - & - & - & $<2.1$ \\
\hline 719 & $6: 40: 51.7$ & $9: 26: 37.1$ & 1.00 & - & - & - & 5278 & - & - & - & $<2.2$ \\
\hline 720 & $6: 40: 51.7$ & $9: 26: 22.2$ & 1.00 & - & - & - & 5279 & - & - & - & $<3.9$ \\
\hline
\end{tabular}


Table 3. (Continued)

\begin{tabular}{|c|c|c|c|c|c|c|c|c|c|c|c|}
\hline$\overline{\mathrm{N}}$ & $\begin{array}{c}\text { RA } \\
{[\mathrm{h} \mathrm{m} \mathrm{s}]}\end{array}$ & $\begin{array}{c}\text { Dec. } \\
{[\mathrm{d} \mathrm{m} \mathrm{s}]}\end{array}$ & $\begin{array}{c}\text { Id. rad. } \\
\text { ["] }\end{array}$ & $\overline{\mathrm{ACIS}}$ & $\overline{2 \text { 2Mass }}$ & Reb.+ & Lamm+ & Flacc+ & $\overline{\text { W56 }}$ & $\overline{\text { Dahm+ }}$ & $\begin{array}{l}\text { Ct. Rate. } \\
{\left[10^{-4} s^{-1}\right]}\end{array}$ \\
\hline$\overline{7721}$ & $6: 40: 51.8$ & 9:39:07.5 & $\overline{\overline{0.50}}$ & $\overline{-1}$ & "06405184+0939074 & $\overline{-}$ & $\overline{5288}$ & $\overline{-}$ & $\overline{1112}$ & $\overline{-}$ & $\overline{<<1.2}$ \\
\hline 722 & $6: 40: 51.8$ & $9: 32: 52.6$ & 0.50 & - & $06405184+0932526$ & - & 5287 & - & - & - & $<0.7$ \\
\hline 723 & $6: 40: 51.9$ & $9: 37: 55.9$ & 0.50 & - & $06405191+0937558$ & - & 5292 & - & - & - & $<0.9$ \\
\hline 724 & $6: 40: 52.0$ & $9: 41: 10.9$ & 1.00 & _- & - & _- & 5294 & - & - & - & $<1.9$ \\
\hline 725 & $6: 40: 52.0$ & $9: 26: 25.3$ & 1.00 & - & - & _- & 5295 & - & - & - & $<2.3$ \\
\hline 726 & $6: 40: 52.0$ & $9: 41: 22.6$ & 1.00 & - & - & - & 5296 & - & - & - & $<2.0$ \\
\hline $727 *$ & $6: 40: 52.1$ & $9: 29: 13.9$ & 0.50 & 106 & $06405210+0929139$ & 3125 & 5303 & - & - & 192 & 12.6 \\
\hline 728 & $6: 40: 52.3$ & $9: 41: 27.3$ & 0.50 & - & $06405228+0941272$ & - & 5309 & - & - & - & $<2.0$ \\
\hline 729 & $6: 40: 52.3$ & $9: 34: 58.7$ & 1.00 & - & - & - & 5310 & - & - & - & $<1.1$ \\
\hline 730 & $6: 40: 52.3$ & $9: 39: 44.3$ & 1.00 & - & - & - & 5311 & - & - & - & $<1.4$ \\
\hline 731 & $6: 40: 52.3$ & $9: 27: 41.7$ & 0.50 & - & $06405233+0927417$ & 3128 & 5313 & - & - & _- & $<1.6$ \\
\hline 732 & $6: 40: 52.5$ & $9: 33: 19.6$ & 0.50 & _- & $06405250+0933196$ & _- & 5315 & - & - & _- & $<0.6$ \\
\hline 733 & $6: 40: 52.6$ & $9: 32: 16.4$ & 1.00 & - & - & - & 5324 & - & - & - & $<0.7$ \\
\hline 734 & $6: 40: 52.6$ & $9: 41: 58.3$ & 1.00 & - & - & - & 5325 & - & - & - & $<2.3$ \\
\hline 735 & $6: 40: 52.6$ & $9: 26: 28.4$ & 1.00 & - & - & - & 5327 & - & - & - & $<2.2$ \\
\hline $736^{*}$ & $6: 40: 52.6$ & $9: 29: 53.5$ & 1.02 & 107 & - & - & - & - & - & - & 11.4 \\
\hline 737 & $6: 40: 52.6$ & 9:26:29.6 & 1.00 & - & - & - & 5328 & - & - & - & $<2.1$ \\
\hline $738^{*}$ & $6: 40: 52.7$ & $9: 28: 55.4$ & 0.50 & - & $06405271+0928554$ & 3140 & 5336 & - & - & - & $<1.3$ \\
\hline 739 & $6: 40: 52.7$ & $9: 38: 16.1$ & 0.50 & _- & $06405271+0938160$ & - & 5337 & - & _- & - & $<1.0$ \\
\hline $740 *$ & $6: 40: 52.7$ & $9: 28: 43.8$ & 0.50 & - & $06405272+0928437$ & - & 5339 & - & - & - & $<1.3$ \\
\hline $741 *$ & $6: 40: 52.8$ & 9:43:00.4 & 0.50 & - & $06405275+0943004$ & 3141 & 5340 & - & - & 197 & $<3.0$ \\
\hline 742 & $6: 40: 52.8$ & $9: 26: 30.3$ & 1.00 & - & - & - & 5341 & - & - & - & $<2.1$ \\
\hline 743 & $6: 40: 52.9$ & $9: 31: 53.5$ & 1.00 & - & - & - & 5346 & - & - & - & $<0.7$ \\
\hline $744 *$ & $6: 40: 52.9$ & $9: 35: 15.5$ & 0.50 & 108 & $06405293+0935155$ & - & - & - & - & - & 11.2 \\
\hline 745 & $6: 40: 52.9$ & $9: 41: 54.1$ & 0.75 & - & $06405294+0941540$ & _- & 5353 & - & - & _- & $<2.1$ \\
\hline $746^{*}$ & $6: 40: 53.0$ & $9: 26: 25.7$ & 0.50 & - & $06405295+0926257$ & 3145 & 5355 & _- & - & 199 & $<2.2$ \\
\hline 747 & $6: 40: 53.0$ & $9: 39: 41.5$ & 1.00 & - & - & - & 5356 & - & - & - & $<1.3$ \\
\hline 748 & $6: 40: 53.0$ & 9:27:19.4 & 0.50 & - & $06405299+0927193$ & - & 5357 & - & - & - & $<1.7$ \\
\hline $749 *$ & $6: 40: 53.1$ & $9: 30: 10.3$ & 0.50 & - & $06405312+0930103$ & - & 5365 & - & - & - & $<1.1$ \\
\hline $750 *$ & $6: 40: 53.2$ & 9:29:54.1 & 0.50 & 109 & $06405321+0929541$ & 3149 & 5370 & - & - & 202 & 1.4 \\
\hline 751 & $6: 40: 53.2$ & $9: 29: 57.1$ & 1.00 & - & - & - & 5372 & - & - & - & $<1.1$ \\
\hline 752 & $6: 40: 53.2$ & $9: 42: 10.8$ & 1.00 & _- & - & _- & 5373 & - & - & _- & $<2.2$ \\
\hline $753 *$ & $6: 40: 53.3$ & $9: 43: 11.6$ & 0.50 & - & $06405328+0943116$ & 3151 & 5376 & _- & - & - & $<5.5$ \\
\hline 754 & $6: 40: 53.4$ & $9: 26: 27.9$ & 1.00 & - & - & - & 5377 & - & - & - & $<2.2$ \\
\hline 755 & $6: 40: 53.4$ & $9: 30: 22.6$ & 0.50 & - & $06405337+0930225$ & - & 5378 & - & - & - & $<1.0$ \\
\hline 756 & $6: 40: 53.4$ & $9: 32: 10.6$ & 0.50 & - & $06405341+0932105$ & - & 5381 & - & - & - & $<0.7$ \\
\hline 757 & $6: 40: 53.4$ & $9: 42: 12.3$ & 1.00 & - & - & - & 5382 & - & - & - & $<2.2$ \\
\hline 758 & $6: 40: 53.5$ & 9:32:06.0 & 1.00 & - & - & - & 5385 & - & - & - & $<0.7$ \\
\hline 759 & $6: 40: 53.5$ & $9: 30: 19.8$ & 1.00 & - & - & _- & 5386 & _- & - & _- & $<1.0$ \\
\hline 760 & $6: 40: 53.6$ & $9: 36: 46.1$ & 0.50 & 110 & $06405362+0936461$ & 3156 & 5392 & - & - & - & 1.2 \\
\hline $761 *$ & $6: 40: 53.6$ & $9: 29: 53.3$ & 0.50 & - & $06405363+0929532$ & - & 5391 & - & - & 203 & $<1.1$ \\
\hline $762 *$ & $6: 40: 53.6$ & $9: 33: 24.7$ & 0.50 & 111 & $06405363+0933247$ & 3157 & 5394 & 54 & 115 & 206 & 37.1 \\
\hline $763^{*}$ & $6: 40: 53.8$ & 9:30:39.0 & 0.50 & 112 & $06405377+0930389$ & - & 5400 & 55 & 116 & - & 22.1 \\
\hline 764 & $6: 40: 53.9$ & 9:40:03.8 & 0.50 & - & $06405391+0940037$ & - & 5405 & - & - & - & $<1.5$ \\
\hline $765^{*}$ & $6: 40: 53.9$ & 9:38:04.9 & 1.16 & 113 & - & - & - & - & - & - & 1.1 \\
\hline 766 & $6: 40: 53.9$ & $9: 40: 32.5$ & 0.50 & - & $06405393+0940324$ & - & 5406 & - & - & - & $<1.6$ \\
\hline 767 & $6: 40: 54.0$ & $9: 32: 11.0$ & 1.00 & _- & - & _- & 5409 & - & - & - & $<0.7$ \\
\hline 768 & $6: 40: 54.0$ & $9: 37: 29.1$ & 0.50 & - & $06405396+0937290$ & - & - & - & - & - & $<0.9$ \\
\hline 769 & $6: 40: 54.0$ & $9: 42: 15.4$ & 0.50 & - & $06405396+0942153$ & 3163 & 5410 & - & - & - & $<2.3$ \\
\hline 770 & $6: 40: 54.0$ & $9: 26: 42.7$ & 1.00 & - & - & - & 5411 & - & - & - & $<2.3$ \\
\hline $771 *$ & $6: 40: 54.1$ & $9: 29: 51.1$ & 0.50 & 114 & $06405411+0929510$ & 3166 & 5413 & 57 & - & 208 & 83.7 \\
\hline 772 & $6: 40: 54.1$ & $9: 28: 17.8$ & 1.00 & - & - & - & 5415 & - & - & - & $<1.4$ \\
\hline 773 & $6: 40: 54.2$ & $9: 28: 30.4$ & 0.50 & - & $06405417+0928303$ & - & 5419 & - & - & - & $<1.4$ \\
\hline 774 & $6: 40: 54.3$ & $9: 42: 50.4$ & 1.00 & _- & _- & _- & 5427 & - & - & _- & $<2.8$ \\
\hline 775 & $6: 40: 54.3$ & $9: 29: 60.0$ & 1.00 & - & - & - & 5428 & - & - & - & $<1.1$ \\
\hline 776 & $6: 40: 54.3$ & $9: 40: 22.7$ & 0.50 & - & $06405430+0940226$ & - & 5430 & - & - & - & $<1.7$ \\
\hline 777 & $6: 40: 54.4$ & $9: 27: 00.5$ & 0.52 & - & $06405437+0927005$ & - & 5432 & - & - & - & $<2.3$ \\
\hline 778 & $6: 40: 54.4$ & $9: 39: 50.5$ & 1.00 & - & - & - & 5434 & - & - & - & $<1.5$ \\
\hline 779 & $6: 40: 54.5$ & $9: 38: 59.4$ & 0.50 & - & $06405446+0938594$ & - & - & - & - & - & $<1.1$ \\
\hline 780 & $6: 40: 54.5$ & $9: 42: 38.2$ & 0.50 & - & $06405449+0942381$ & 3185 & 5438 & - & - & - & $<2.6$ \\
\hline
\end{tabular}


Table 3. (Continued)

\begin{tabular}{|c|c|c|c|c|c|c|c|c|c|c|c|}
\hline$\overline{\mathrm{N}}$ & $\begin{array}{c}\mathrm{RA} \\
{[\mathrm{h} \mathrm{m} \mathrm{s}]}\end{array}$ & $\begin{array}{c}\text { Dec. } \\
{[\mathrm{d} \mathrm{m} \mathrm{s}]}\end{array}$ & $\begin{array}{c}\text { Id. rad. } \\
\text { ["] }\end{array}$ & $\overline{\mathrm{ACIS}}$ & 2Mass & Reb.+ & $\overline{\mathrm{Lamm+}}$ & Flacc+ & W56 & $\overline{\overline{\text { Dahm+ }}}$ & $\begin{array}{l}\text { Ct. Rate. } \\
{\left[10^{-4} s^{-1}\right]}\end{array}$ \\
\hline 781 & $6: 40: 54.5$ & $9: 42: 55.3$ & 1.00 & $\overline{-}$ & $\overline{-1}$ & $=$ & $\overline{5441}$ & $=$ & 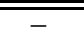 & $=$ & 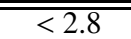 \\
\hline 782 & $6: 40: 54.6$ & $9: 31: 07.7$ & 0.50 & - & $06405455+0931076$ & - & 5440 & - & - & - & $<0.8$ \\
\hline 783 & $6: 40: 54.6$ & $9: 36: 25.9$ & 1.00 & - & - & - & 5444 & - & - & - & $<0.7$ \\
\hline 784 & $6: 40: 54.6$ & 9:43:07.6 & 1.00 & - & - & - & 5447 & - & - & - & $<3.9$ \\
\hline 785 & $6: 40: 54.7$ & $9: 39: 58.1$ & 1.00 & - & - & - & 5449 & - & - & - & $<1.5$ \\
\hline $786^{*}$ & $6: 40: 54.7$ & $9: 31: 59.0$ & 1.11 & 115 & - & - & - & - & - & - & 0.7 \\
\hline 787 & $6: 40: 54.7$ & $9: 42: 50.1$ & 0.50 & - & $06405471+0942500$ & - & 5453 & - & - & - & $<2.8$ \\
\hline 788 & $6: 40: 54.8$ & $9: 37: 58.7$ & 0.50 & - & $06405480+0937587$ & - & - & - & - & - & $<0.9$ \\
\hline 789 & $6: 40: 55.0$ & $9: 42: 11.5$ & 1.00 & - & - & - & 5469 & - & - & - & $<2.3$ \\
\hline 790 & $6: 40: 55.1$ & $9: 32: 39.6$ & 0.50 & - & $06405505+0932395$ & - & 5472 & - & - & - & $<0.6$ \\
\hline 791 & $6: 40: 55.1$ & $9: 42: 58.6$ & 1.00 & - & - & - & 5474 & - & - & - & $<2.9$ \\
\hline $792 *$ & $6: 40: 55.1$ & 9:33:18.7 & 0.50 & 116 & $06405514+0933186$ & 3199 & 5475 & - & - & 212 & 1.2 \\
\hline $793^{*}$ & $6: 40: 55.2$ & $9: 30: 16.7$ & 1.39 & 117 & - & - & - & - & - & - & 1.1 \\
\hline $794 *$ & $6: 40: 55.2$ & $9: 41: 28.9$ & 0.50 & - & $06405522+0941289$ & 3202 & 5482 & - & - & - & $<2.0$ \\
\hline 795 & $6: 40: 55.3$ & $9: 42: 36.8$ & 0.50 & - & $06405526+0942367$ & - & 5484 & - & - & - & $<2.6$ \\
\hline $796^{*}$ & $6: 40: 55.3$ & $9: 33: 24.9$ & 0.50 & 118 & $06405527+0933249$ & 3206 & 5486 & - & - & - & 1.6 \\
\hline 797 & $6: 40: 55.3$ & 9:30:07.8 & 0.50 & - & $06405530+0930078$ & 3208 & 5490 & - & - & 215 & $<1.0$ \\
\hline $798^{*}$ & $6: 40: 55.3$ & $9: 39: 58.8$ & 0.50 & 119 & $06405532+0939588$ & - & 5491 & - & - & 214 & 12.1 \\
\hline 799 & $6: 40: 55.4$ & $9: 39: 52.1$ & 1.00 & - & - & - & 5493 & - & - & - & $<1.5$ \\
\hline 800 & $6: 40: 55.4$ & $9: 26: 23.1$ & 1.00 & - & - & - & 5496 & - & - & - & $<3.6$ \\
\hline 801 & $6: 40: 55.4$ & $9: 30: 18.0$ & 0.50 & - & $06405540+0930180$ & - & 5497 & - & - & - & $<1.0$ \\
\hline $802 *$ & $6: 40: 55.4$ & $9: 37: 23.7$ & 0.50 & 120 & $06405542+0937237$ & 3211 & 5498 & 59 & 119 & 217 & 39.2 \\
\hline $803 *$ & $6: 40: 55.5$ & $9: 31: 21.8$ & 0.50 & 121 & $06405548+0931218$ & 3212 & 5501 & - & 120 & - & 1.8 \\
\hline $804 *$ & $6: 40: 55.5$ & $9: 38: 39.6$ & 0.50 & 122 & $06405554+0938396$ & 3213 & 5502 & - & - & - & 1.4 \\
\hline 805 & $6: 40: 55.6$ & $9: 28: 26.5$ & 1.00 & - & - & - & 5503 & - & - & - & $<1.8$ \\
\hline $806^{*}$ & $6: 40: 55.6$ & $9: 38: 24.9$ & 0.50 & 123 & $06405561+0938249$ & 3214 & 5505 & - & - & 219 & 2.0 \\
\hline $807 *$ & $6: 40: 55.8$ & $9: 40: 18.2$ & 0.50 & 124 & $06405577+0940181$ & 3218 & 5518 & 60 & - & 223 & 49.3 \\
\hline 808 & $6: 40: 55.8$ & $9: 31: 48.0$ & 1.00 & - & - & - & 5519 & - & - & - & $<0.7$ \\
\hline $809 *$ & $6: 40: 55.8$ & $9: 36: 06.2$ & 0.50 & 125 & $06405579+0936061$ & - & 5520 & - & - & - & 1.1 \\
\hline 810 & $6: 40: 55.8$ & $9: 26: 29.7$ & 1.00 & - & - & - & 5522 & - & - & - & $<2.6$ \\
\hline $811^{*}$ & $6: 40: 55.9$ & $9: 37: 17.8$ & 0.50 & 126 & $06405586+0937177$ & 3221 & 5526 & - & - & 225 & 3.4 \\
\hline 812 & $6: 40: 55.9$ & 9:36:59.0 & 0.50 & - & $06405586+0936590$ & 3222 & 5525 & - & - & - & $<0.8$ \\
\hline 813 & $6: 40: 55.9$ & $9: 31: 32.4$ & 0.50 & - & $06405590+0931324$ & - & 5528 & - & - & - & $<0.7$ \\
\hline 814 & $6: 40: 56.0$ & $9: 30: 02.4$ & 0.71 & - & $06405603+0930024$ & - & 5532 & $63 \dagger$ & - & - & $<1.0$ \\
\hline 815 & $6: 40: 56.0$ & $9: 28: 12.9$ & 1.00 & - & - & - & 5531 & - & - & - & $<3.5$ \\
\hline 816 & $6: 40: 56.1$ & 9:43:02.6 & 0.50 & - & $06405606+0943025$ & 3226 & 5534 & - & - & - & $<3.2$ \\
\hline $817 *$ & $6: 40: 56.1$ & $9: 42: 54.1$ & 0.50 & - & $06405607+0942540$ & 3228 & 5533 & - & - & 226 & $<2.8$ \\
\hline 818 & $6: 40: 56.1$ & $9: 30: 16.9$ & 0.67 & - & $06405614+0930169$ & - & 5537 & $63 \dagger$ & - & - & $<1.0$ \\
\hline $819^{*}$ & $6: 40: 56.2$ & 9:36:30.9 & 0.50 & 127 & $06405616+0936309$ & - & 5538 & - & 122 & 227 & 22.0 \\
\hline 820 & $6: 40: 56.2$ & $9: 32: 22.9$ & 0.50 & - & $06405621+0932229$ & 3232 & 5540 & - & - & - & $<0.6$ \\
\hline 821 & $6: 40: 56.2$ & $9: 40: 45.4$ & 1.00 & - & - & - & 5543 & - & - & - & $<1.8$ \\
\hline $822 *$ & $6: 40: 56.2$ & $9: 39: 32.7$ & 0.50 & 129 & $06405624+0939327$ & 3233 & 5542 & - & - & 228 & 2.3 \\
\hline 823 & $6: 40: 56.2$ & $9: 40: 37.9$ & 0.50 & 128 & $06405625+0940378$ & 3234 & 5544 & - & - & - & 1.5 \\
\hline 824 & $6: 40: 56.3$ & $9: 28: 27.9$ & 0.71 & - & $06405626+0928279$ & - & 5547 & - & - & - & $<3.5$ \\
\hline 825 & $6: 40: 56.3$ & $9: 39: 30.5$ & 1.00 & - & - & - & 5545 & - & - & - & $<2.3$ \\
\hline 826 & $6: 40: 56.3$ & $9: 37: 30.2$ & 0.50 & - & $06405627+0937302$ & - & - & - & - & - & $<0.8$ \\
\hline 827 & $6: 40: 56.3$ & 9:34:18.0 & 0.50 & 130 & $06405630+0934180$ & 3236 & 5548 & - & - & 229 & 5.2 \\
\hline $828 *$ & $6: 40: 56.4$ & $9: 30: 50.0$ & 0.50 & - & $06405638+0930499$ & - & 5550 & - & - & - & $<0.8$ \\
\hline $829 *$ & $6: 40: 56.4$ & $9: 35: 53.3$ & 0.50 & 131 & $06405639+0935533$ & 3240 & 5551 & - & 123 & 230 & 1.7 \\
\hline 830 & $6: 40: 56.4$ & $9: 34: 44.2$ & 0.57 & - & $06405641+0934442$ & 3241 & 5552 & - & - & - & $<1.2$ \\
\hline 831 & $6: 40: 56.5$ & $9: 41: 19.3$ & 1.00 & - & - & - & 5554 & - & - & - & $<2.0$ \\
\hline 832 & $6: 40: 56.5$ & $9: 34: 38.0$ & 1.00 & - & - & - & 5555 & - & - & - & $<1.3$ \\
\hline $833^{*}$ & $6: 40: 56.5$ & $9: 29: 53.9$ & 0.50 & 132 & $06405649+0929538$ & 3244 & 5558 & - & - & 231 & 3.7 \\
\hline 834 & $6: 40: 56.5$ & $9: 31: 30.9$ & 0.50 & - & $06405652+0931308$ & - & 5559 & - & - & - & $<0.7$ \\
\hline 835 & $6: 40: 56.6$ & $9: 39: 43.3$ & 0.50 & - & $06405658+0939432$ & 3248 & 5560 & - & 124 & - & $<1.5$ \\
\hline 836 & $6: 40: 56.6$ & $9: 32: 19.6$ & 0.50 & - & $06405664+0932196$ & - & 5567 & - & - & - & $<0.6$ \\
\hline 837 & $6: 40: 56.7$ & 9:30:03.0 & 0.50 & - & $06405665+0930029$ & - & 5568 & $63 \dagger$ & - & - & $<1.0$ \\
\hline 838 & $6: 40: 56.7$ & $9: 38: 10.2$ & 0.50 & - & $06405674+0938101$ & - & 5570 & - & - & - & $<1.0$ \\
\hline 839 & $6: 40: 56.8$ & $9: 41: 27.4$ & 0.50 & - & $06405675+0941273$ & - & 5572 & - & - & - & $<2.1$ \\
\hline $840^{*}$ & $6: 40: 56.8$ & $9: 30: 15.1$ & 0.50 & 133 & $06405677+0930150$ & 3252 & 5571 & $63 \dagger$ & 127 & 232 & 20.8 \\
\hline
\end{tabular}


Table 3. (Continued)

\begin{tabular}{|c|c|c|c|c|c|c|c|c|c|c|c|}
\hline$\overline{\mathrm{N}}$ & $\begin{array}{c}\mathrm{RA} \\
{[\mathrm{h} \mathrm{m} \mathrm{s}]}\end{array}$ & $\begin{array}{c}\text { Dec. } \\
{[\mathrm{d} \mathrm{m} \mathrm{s}]}\end{array}$ & $\begin{array}{c}\text { Id. rad. } \\
\text { ["] }\end{array}$ & $\overline{\mathrm{ACIS}}$ & 2Mass & Reb.+ & Lamm+ & Flacc+ & $\overline{\text { W56 }}$ & $\overline{\overline{\text { Dahm+ }}}$ & $\begin{array}{l}\text { Ct. Rate. } \\
{\left[10^{-4} s^{-1}\right]}\end{array}$ \\
\hline $841^{*}$ & $6: 40: 56.8$ & 9:29:59.7 & 0.50 & 134 & $06405678+0929596$ & $\overline{-}$ & $\overline{5574}$ & $63 \dagger$ & $\overline{-}$ & 233 & $\overline{2.0}$ \\
\hline 842 & $6: 40: 56.8$ & $9: 30: 05.3$ & 0.51 & - & $06405678+0930052$ & - & 5576 & $63 \dagger$ & - & - & $<1.1$ \\
\hline $843 *$ & $6: 40: 56.8$ & $9: 37: 49.0$ & 0.50 & 135 & $06405679+0937490$ & 3253 & 5575 & 62 & 126 & 234 & 92.9 \\
\hline 844 & $6: 40: 56.8$ & $9: 28: 23.4$ & 1.00 & - & - & - & 5577 & - & - & - & $<2.9$ \\
\hline 845 & $6: 40: 56.8$ & $9: 30: 14.6$ & 1.00 & - & - & - & 5579 & - & - & - & $<18.5$ \\
\hline 846 & $6: 40: 56.9$ & $9: 34: 27.2$ & 0.65 & - & $06405689+0934272$ & _- & 5587 & - & - & - & $<0.9$ \\
\hline 847 & $6: 40: 56.9$ & $9: 31: 52.9$ & 0.50 & - & $06405689+0931528$ & - & 5584 & - & - & - & $<0.7$ \\
\hline 848 & $6: 40: 57.0$ & $9: 29: 38.5$ & 0.50 & - & $06405699+0929384$ & 3258 & 5590 & - & - & - & $<2.1$ \\
\hline $849 *$ & $6: 40: 57.0$ & $9: 33: 01.3$ & 0.50 & 136 & $06405699+0933013$ & 3260 & 5591 & - & - & 237 & 14.9 \\
\hline 850 & $6: 40: 57.1$ & 9:32:27.4 & 0.50 & - & $06405708+0932273$ & - & 5595 & - & - & - & $<0.6$ \\
\hline 851 & $6: 40: 57.2$ & $9: 31: 03.0$ & 0.54 & - & $06405723+0931029$ & - & 5597 & - & - & - & $<0.8$ \\
\hline 852 & $6: 40: 57.3$ & $9: 26: 52.8$ & 0.50 & - & $06405729+0926527$ & - & 5602 & - & - & - & $<2.0$ \\
\hline 853 & $6: 40: 57.4$ & $9: 40: 42.7$ & 0.50 & - & $06405740+0940426$ & - & 5606 & - & - & - & $<1.9$ \\
\hline $854 *$ & $6: 40: 57.5$ & 9:29:23.4 & 0.50 & 137 & $06405745+0929234$ & 3268 & 5612 & - & - & 238 & 11.2 \\
\hline $855^{*}$ & $6: 40: 57.5$ & $9: 26: 17.1$ & 0.50 & 138 & $06405745+0926170$ & 3266 & 5610 & - & - & - & 2.9 \\
\hline $856 *$ & $6: 40: 57.5$ & $9: 35: 14.7$ & 0.50 & - & $06405747+0935147$ & - & 5613 & - & - & 239 & $<0.7$ \\
\hline 857 & $6: 40: 57.5$ & $9: 40: 14.2$ & 1.00 & - & - & - & 5615 & - & - & - & $<1.6$ \\
\hline $858 *$ & $6: 40: 57.6$ & $9: 37: 07.5$ & 0.50 & 139 & $06405761+0937075$ & 3270 & 5622 & - & - & 240 & 1.5 \\
\hline $859 *$ & $6: 40: 57.7$ & $9: 31: 50.1$ & 0.50 & 140 & $06405766+0931501$ & 3272 & 5624 & - & - & 241 & 2.4 \\
\hline $860 *$ & $6: 40: 57.7$ & $9: 36: 08.2$ & 0.50 & 141 & $06405767+0936082$ & - & - & - & - & - & 7.8 \\
\hline 861 & $6: 40: 57.8$ & 9:39:53.8 & 0.50 & - & $06405775+0939537$ & - & - & - & - & - & $<1.4$ \\
\hline $862 *$ & $6: 40: 57.8$ & $9: 30: 50.3$ & 0.50 & 142 & $06405777+0930502$ & 3276 & - & $64 \dagger$ & - & 242 & 40.9 \\
\hline $863 *$ & $6: 40: 57.8$ & $9: 41: 20.2$ & 0.50 & 143 & $06405783+0941201$ & 3278 & - & - & 129 & 244 & 6.5 \\
\hline $864 *$ & $6: 40: 57.9$ & 9:27:09.0 & 3.41 & 144 & - & - & - & - & - & - & 1.8 \\
\hline $865^{*}$ & $6: 40: 58.0$ & $9: 36: 39.3$ & 1.10 & 145 & - & - & - & - & - & - & 0.9 \\
\hline 866 & $6: 40: 58.0$ & $9: 36: 15.1$ & 1.00 & - & - & - & - & - & - & - & $<0.7$ \\
\hline $867 *$ & $6: 40: 58.0$ & $9: 41: 31.4$ & 0.50 & - & $06405799+0941314$ & 3282 & - & - & - & 245 & $<2.2$ \\
\hline 868 & $6: 40: 58.0$ & $9: 40: 49.3$ & 0.50 & - & $06405803+0940493$ & - & - & - & - & - & $<1.8$ \\
\hline $869^{*}$ & $6: 40: 58.1$ & $9: 36: 53.4$ & 0.50 & 146 & $06405809+0936533$ & 3284 & - & - & - & 246 & 2.4 \\
\hline 870 & $6: 40: 58.3$ & $9: 42: 34.7$ & 0.69 & - & $06405832+0942347$ & - & - & - & - & - & $<2.7$ \\
\hline $871 *$ & $6: 40: 58.3$ & $9: 37: 56.8$ & 0.50 & 147 & $06405834+0937567$ & - & - & - & - & - & 3.5 \\
\hline $872 *$ & $6: 40: 58.4$ & $9: 27: 25.0$ & 0.50 & 148 & $06405839+0927250$ & 3289 & - & - & - & 250 & 6.5 \\
\hline 873 & $6: 40: 58.4$ & $9: 28: 46.9$ & 0.50 & - & $06405840+0928468$ & - & - & - & - & - & $<1.5$ \\
\hline 874 & $6: 40: 58.4$ & $9: 30: 58.0$ & 0.66 & - & $06405842+0930579$ & - & - & $64 \dagger$ & - & - & $<1.9$ \\
\hline 875 & $6: 40: 58.4$ & $9: 37: 44.5$ & 0.50 & - & $06405843+0937445$ & - & - & - & - & - & $<0.9$ \\
\hline 876 & $6: 40: 58.4$ & $9: 34: 52.5$ & 0.50 & - & $06405843+0934525$ & - & - & - & - & - & $<0.6$ \\
\hline 877 & $6: 40: 58.5$ & 9:42:08.3 & 0.50 & - & $06405845+0942083$ & - & - & - & - & - & $<2.4$ \\
\hline $878 *$ & $6: 40: 58.5$ & $9: 33: 31.7$ & 0.50 & 149 & $06405851+0933317$ & - & - & 65 & 132 & - & 324.0 \\
\hline 879 & $6: 40: 58.6$ & $9: 32: 22.9$ & 0.50 & - & $06405859+0932229$ & - & - & - & - & - & $<0.6$ \\
\hline $880 *$ & $6: 40: 58.6$ & $9: 36: 39.4$ & 0.50 & 150 & $06405861+0936393$ & - & - & - & - & - & 1.0 \\
\hline $881 *$ & $6: 40: 58.7$ & $9: 36: 13.3$ & 0.50 & 151 & $06405867+0936132$ & 3302 & - & - & - & 251 & 77.0 \\
\hline 882 & $6: 40: 58.7$ & $9: 30: 43.9$ & 0.50 & - & $06405874+0930439$ & - & - & - & - & - & $<1.5$ \\
\hline 883 & $6: 40: 58.7$ & 9:38:05.8 & 1.50 & - & - & 3303 & - & - & - & - & $<1.0$ \\
\hline 884 & $6: 40: 58.7$ & $9: 37: 45.5$ & 1.50 & - & - & 3304 & - & - & - & - & $<0.8$ \\
\hline $885^{*}$ & $6: 40: 58.8$ & 9:34:05.4 & 1.00 & 152 & - & - & - & - & - & - & 1.8 \\
\hline $886^{*}$ & $6: 40: 58.8$ & $9: 39: 18.8$ & 0.50 & 155 & $06405882+0939187$ & 3300 & - & 66 & - & 252 & 20.4 \\
\hline $887 *$ & $6: 40: 58.8$ & $9: 30: 57.3$ & 0.50 & 154 & $06405884+0930573$ & 3307 & - & - & - & 253 & 43.2 \\
\hline $888^{*}$ & 6:40:58.9 & $9: 36: 46.3$ & 0.50 & 156 & $06405886+0936462$ & - & - & - & - & - & 3.1 \\
\hline $889 *$ & $6: 40: 58.9$ & $9: 42: 25.5$ & 0.50 & 153 & $06405888+0942255$ & 3298 & - & - & - & - & 2.0 \\
\hline $890 *$ & $6: 40: 58.9$ & $9: 28: 52.8$ & 0.50 & 157 & $06405891+0928528$ & 3305 & - & - & - & 254 & 6.6 \\
\hline 891 & $6: 40: 58.9$ & 9:36:01.1 & 0.75 & - & $06405892+0936011$ & - & - & - & - & - & $<0.7$ \\
\hline 892 & $6: 40: 58.9$ & $9: 42: 15.5$ & 0.50 & - & $06405893+0942155$ & 3299 & - & - & - & - & $<2.4$ \\
\hline 893 & $6: 40: 58.9$ & $9: 26: 33.5$ & 0.50 & - & $06405894+0926335$ & - & - & - & - & - & $<2.1$ \\
\hline $894 *$ & $6: 40: 59.1$ & $9: 33: 22.2$ & 0.50 & 158 & $06405905+0933222$ & - & - & - & - & 257 & 1.0 \\
\hline 895 & $6: 40: 59.1$ & 9:33:01.9 & 0.50 & - & $06405906+0933019$ & - & - & - & - & - & $<0.7$ \\
\hline 896 & $6: 40: 59.1$ & 9:36:15.1 & 1.00 & - & - & - & - & - & - & - & $<0.7$ \\
\hline 897 & $6: 40: 59.2$ & $9: 35: 45.3$ & 0.50 & - & $06405915+0935452$ & - & - & - & - & - & $<0.7$ \\
\hline 898 & $6: 40: 59.2$ & $9: 37: 28.1$ & 0.50 & - & $06405919+0937281$ & - & - & - & - & - & $<0.8$ \\
\hline $899 *$ & $6: 40: 59.2$ & $9: 31: 50.3$ & 0.50 & 159 & $06405923+0931503$ & 3317 & - & - & - & - & 3.2 \\
\hline $900 *$ & $6: 40: 59.3$ & $9: 33: 25.0$ & 0.50 & 160 & $06405926+0933250$ & - & - & - & - & 258 & 0.9 \\
\hline
\end{tabular}


Table 3. (Continued)

\begin{tabular}{|c|c|c|c|c|c|c|c|c|c|c|c|}
\hline$\overline{\mathrm{N}}$ & $\begin{array}{c}\text { RA } \\
{[\mathrm{h} \mathrm{m} \mathrm{s}]}\end{array}$ & $\begin{array}{c}\text { Dec. } \\
{[\mathrm{d} \mathrm{m} \mathrm{s}]}\end{array}$ & $\begin{array}{c}\text { Id. rad. } \\
\text { ["] }\end{array}$ & $\overline{\mathrm{ACIS}}$ & 2Mass & Reb.+ & $\overline{\text { Lamm+ }}$ & Flacc+ & W56 & $\overline{\text { Dahm+ }}$ & $\begin{array}{l}\text { Ct. Rate. } \\
{\left[10^{-4} s^{-1}\right]}\end{array}$ \\
\hline $901^{*}$ & $6: 40: 59.3$ & $\overline{79: 35: 52.4}$ & 0.50 & $\overline{161}$ & $06405930+0935523$ & 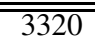 & $\overline{-1}$ & - & $\overline{-}$ & $\overline{-1}$ & 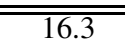 \\
\hline 902 & $6: 40: 59.3$ & $9: 33: 58.3$ & 0.50 & - & $06405930+0933583$ & - & - & - & - & - & $<0.6$ \\
\hline 903 & $6: 40: 59.3$ & $9: 42: 40.3$ & 0.50 & - & $06405931+0942402$ & - & - & - & - & - & $<2.7$ \\
\hline $904 *$ & $6: 40: 59.3$ & $9: 35: 56.9$ & 1.00 & 162 & - & - & - & - & - & - & 6.8 \\
\hline $905^{*}$ & $6: 40: 59.4$ & $9: 33: 33.4$ & 0.50 & 163 & $06405936+0933333$ & - & - & - & - & - & 21.1 \\
\hline $906^{*}$ & $6: 40: 59.4$ & $9: 30: 59.6$ & 0.50 & - & $06405940+0930595$ & - & 5627 & - & - & - & $<0.9$ \\
\hline $907 *$ & $6: 40: 59.4$ & $9: 27: 53.5$ & 0.50 & _- & $06405942+0927534$ & 3326 & 5628 & _- & _- & - & $<1.6$ \\
\hline $908 *$ & $6: 40: 59.5$ & $9: 29: 51.7$ & 0.50 & 164 & $06405949+0929517$ & 3329 & 5630 & - & - & 262 & 4.6 \\
\hline $909 *$ & $6: 40: 59.5$ & $9: 36: 10.5$ & 0.50 & 165 & $06405952+0936105$ & - & - & - & - & - & 12.8 \\
\hline 910 & $6: 40: 59.5$ & $9: 27: 31.9$ & 0.50 & - & $06405953+0927319$ & - & 5629 & - & - & - & $<1.9$ \\
\hline 911 & $6: 40: 59.5$ & $9: 34: 43.7$ & 1.00 & - & - & - & 5631 & - & - & - & $<0.7$ \\
\hline $912 *$ & $6: 40: 59.5$ & $9: 35: 10.9$ & 0.50 & 167 & $06405954+0935109$ & 3330 & 5633 & 71 & 136 & 260 & 107.2 \\
\hline $913 *$ & $6: 40: 59.6$ & $9: 35: 51.7$ & 1.00 & 168 & - & - & - & - & - & - & 1.3 \\
\hline $914 *$ & $6: 40: 59.6$ & 9:39:06.3 & 0.50 & 166 & $06405955+0939063$ & 3331 & - & - & - & 261 & 1.4 \\
\hline $915^{*}$ & $6: 40: 59.6$ & $9: 36: 57.6$ & 0.50 & 169 & $06405962+0936575$ & 3333 & 5634 & - & - & 263 & 1.0 \\
\hline $916^{*}$ & $6: 40: 59.7$ & $9: 28: 43.8$ & 0.50 & 170 & $06405968+0928438$ & 3336 & 5638 & $73+$ & - & 265 & 2.7 \\
\hline 917 & $6: 40: 59.7$ & $9: 28: 49.8$ & 1.00 & - & - & - & 5639 & - & - & - & $<41.2$ \\
\hline 918 & $6: 40: 59.7$ & $9: 34: 22.3$ & 0.50 & - & $06405972+0934222$ & - & 5640 & - & - & - & $<1.2$ \\
\hline 919 & $6: 40: 59.8$ & $9: 27: 29.2$ & 1.00 & - & - & - & 5644 & - & - & - & $<1.9$ \\
\hline 920 & $6: 40: 59.8$ & $9: 35: 59.2$ & 0.50 & - & $06405975+0935592$ & - & 5643 & - & - & - & $<0.7$ \\
\hline 921 & $6: 40: 59.8$ & $9: 36: 33.3$ & 0.50 & - & $06405981+0936332$ & - & - & - & - & - & $<0.7$ \\
\hline 922 & $6: 40: 59.8$ & $9: 42: 06.1$ & 1.00 & - & - & - & 5646 & - & - & - & $<2.3$ \\
\hline 923 & $6: 40: 59.9$ & $9: 35: 17.2$ & 1.00 & - & - & - & 5649 & - & - & - & $<0.6$ \\
\hline 924 & $6: 40: 60.0$ & $9: 35: 01.0$ & 0.50 & 171 & $06405998+0935009$ & - & 5652 & - & - & - & 0.9 \\
\hline $925 *$ & $6: 41: 00.0$ & $9: 28: 50.0$ & 0.50 & 172 & $06405999+0928500$ & 3343 & 5653 & $73 \dagger$ & - & 268 & 122.9 \\
\hline 926 & $6: 41: 00.0$ & $9: 32: 23.3$ & 0.65 & - & $06410002+0932233$ & - & - & - & - & - & $<1.3$ \\
\hline 927 & $6: 41: 00.2$ & $9: 42: 37.3$ & 1.00 & - & - & - & 5656 & - & - & - & $<2.6$ \\
\hline 928 & $6: 41: 00.2$ & 9:39:07.0 & 0.50 & - & $06410022+0939070$ & 3349 & 5657 & - & - & 269 & $<1.2$ \\
\hline $929 *$ & $6: 41: 00.3$ & $9: 36: 31.2$ & 0.50 & 173 & $06410025+0936311$ & - & 5659 & - & - & - & 0.9 \\
\hline $930 *$ & $6: 41: 00.3$ & $9: 28: 34.1$ & 0.50 & - & $06410028+0928341$ & - & 5661 & - & - & 270 & $<1.6$ \\
\hline $931 *$ & $6: 41: 00.3$ & $9: 35: 59.1$ & 0.50 & 174 & $06410028+0935591$ & - & - & - & - & - & 3.7 \\
\hline $932 *$ & $6: 41: 00.3$ & $9: 34: 24.3$ & 0.50 & - & $06410030+0934243$ & - & 5663 & - & - & - & $<1.9$ \\
\hline 933 & $6: 41: 00.3$ & $9: 30: 16.9$ & 0.50 & - & $06410032+0930169$ & 3356 & 5664 & - & - & - & $<0.9$ \\
\hline $934 *$ & 6:41:00.4 & $9: 41: 15.3$ & 0.50 & 175 & $06410040+0941153$ & - & 5667 & - & - & - & 1.8 \\
\hline 935 & 6:41:00.4 & $9: 41: 29.3$ & 0.50 & - & $06410041+0941293$ & - & 5669 & - & - & - & $<1.9$ \\
\hline $936^{*}$ & $6: 41: 00.4$ & $9: 41: 20.2$ & 0.50 & - & $06410044+0941201$ & - & 5672 & - & - & - & $<1.9$ \\
\hline $937 *$ & $6: 41: 00.5$ & $9: 29: 16.0$ & 0.50 & 176 & $06410051+0929159$ & 3359 & 5677 & $75 \dagger$ & - & 271 & 97.9 \\
\hline 938 & $6: 41: 00.5$ & $9: 41: 44.4$ & 0.50 & - & $06410054+0941444$ & - & 5678 & - & - & - & $<2.0$ \\
\hline $939 *$ & $6: 41: 00.5$ & $9: 36: 12.6$ & 1.00 & 177 & - & 3362 & 5679 & - & - & - & 2.5 \\
\hline $940 *$ & $6: 41: 00.6$ & $9: 36: 10.3$ & 0.50 & 178 & $06410063+0936103$ & - & 5680 & - & - & - & 11.7 \\
\hline 941 & 6:41:00.8 & 9:28:39.9 & 1.00 & - & - & - & 5688 & - & - & - & $<1.5$ \\
\hline $942 *$ & $6: 41: 00.8$ & $9: 30: 14.2$ & 0.50 & - & $06410082+0930142$ & 3371 & 5690 & - & - & 275 & $<0.9$ \\
\hline $943 *$ & $6: 41: 01.0$ & $9: 32: 44.4$ & 0.50 & 179 & $06410098+0932444$ & 3373 & 5692 & 78 & 139 & 276 & 83.4 \\
\hline $944 *$ & $6: 41: 01.0$ & $9: 29: 37.5$ & 1.17 & 180 & - & - & - & - & - & - & 1.2 \\
\hline 945 & $6: 41: 01.1$ & $9: 27: 32.7$ & 1.00 & - & - & - & 5695 & - & - & - & $<1.8$ \\
\hline $946^{*}$ & $6: 41: 01.1$ & $9: 34: 52.2$ & 0.50 & 181 & $06410111+0934522$ & - & 5696 & $79 \dagger$ & - & 277 & 28.0 \\
\hline 947 & 6:41:01.1 & $9: 29: 15.7$ & 0.50 & - & $06410114+0929157$ & - & 5697 & $75 \dagger$ & - & - & $<1.3$ \\
\hline 948 & $6: 41: 01.2$ & $9: 41: 47.7$ & 0.50 & - & $06410116+0941476$ & - & 5698 & - & - & - & $<2.0$ \\
\hline $949 *$ & 6:41:01.2 & 9:36:09.5 & 0.50 & 182 & $06410123+0936095$ & - & - & - & - & - & 6.7 \\
\hline $950 *$ & 6:41:01.3 & $9: 34: 52.6$ & 0.50 & 183 & $06410133+0934526$ & - & 5701 & $79 \dagger$ & - & 278 & 23.3 \\
\hline 951 & 6:41:01.4 & 9:34:07.8 & 1.00 & - & - & - & - & - & - & - & $<57.6$ \\
\hline 952 & $6: 41: 01.4$ & $9: 27: 41.4$ & 1.00 & - & - & - & 5702 & - & - & - & $<1.8$ \\
\hline $953^{*}$ & $6: 41: 01.4$ & $9: 34: 08.1$ & 0.50 & 184 & $06410141+0934081$ & 3378 & 5704 & 80 & - & - & 63.5 \\
\hline 954 & 6:41:01.4 & $9: 38: 50.6$ & 0.50 & - & $06410144+0938506$ & - & - & - & - & - & $<1.1$ \\
\hline 955 & $6: 41: 01.5$ & $9: 40: 10.5$ & 0.50 & - & $06410146+0940104$ & - & - & - & - & - & $<1.5$ \\
\hline $956^{*}$ & $6: 41: 01.6$ & $9: 28: 13.1$ & 0.50 & 185 & $06410155+0928130$ & - & 5709 & - & 145 & - & 27.7 \\
\hline 957 & 6:41:01.6 & $9: 34: 33.0$ & 0.50 & - & $06410156+0934329$ & - & 5710 & - & - & - & $<2.7$ \\
\hline 958 & 6:41:01.6 & $9: 28: 41.2$ & 0.50 & - & $06410158+0928411$ & - & 5712 & - & - & - & $<1.4$ \\
\hline 959 & $6: 41: 01.6$ & $9: 41: 39.4$ & 0.50 & - & $06410159+0941394$ & - & 5713 & - & - & - & $<2.1$ \\
\hline $960 *$ & $6: 41: 01.6$ & $9: 37: 28.8$ & 0.50 & - & $06410160+0937287$ & 3386 & 5714 & - & - & 279 & $<0.8$ \\
\hline
\end{tabular}


Table 3. (Continued)

\begin{tabular}{|c|c|c|c|c|c|c|c|c|c|c|c|}
\hline$\overline{\mathrm{N}}$ & $\begin{array}{c}\text { RA } \\
{[\mathrm{h} \mathrm{m} \mathrm{s}]}\end{array}$ & $\begin{array}{c}\text { Dec. } \\
{[\mathrm{d} \mathrm{m} \mathrm{s}]}\end{array}$ & $\begin{array}{c}\text { Id. rad. } \\
\text { ["] }\end{array}$ & $\overline{\mathrm{ACIS}}$ & 2Mass & $\overline{\text { Reb.+ }}$ & $\overline{\text { Lamm+ }}$ & Flacc+ & $\overline{\text { W56 }}$ & $\overline{\mathrm{Dahm+}}$ & $\begin{array}{l}\text { Ct. Rate. } \\
{\left[10^{-4} s^{-1}\right]}\end{array}$ \\
\hline$\overline{\overline{961}}$ & 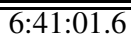 & $9: 40: 39.8$ & $\overline{0.61}$ & - & "06410162+0940398 & $\overline{-1}$ & $\overline{5715}$ & $\overline{-1}$ & $\overline{-1}$ & 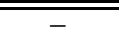 & $\overline{c<1.7}$ \\
\hline 962 & $6: 41: 01.7$ & $9: 27: 23.4$ & 1.00 & - & - & - & 5717 & - & - & - & $<2.0$ \\
\hline 963 & $6: 41: 01.7$ & 9:27:08.4 & 1.00 & - & - & - & 5719 & - & - & - & $<2.1$ \\
\hline 964 & $6: 41: 01.7$ & $9: 38: 25.3$ & 1.00 & - & - & - & 5720 & - & - & - & $<1.0$ \\
\hline $965^{*}$ & $6: 41: 01.7$ & $9: 42: 43.0$ & 0.50 & - & $06410171+0942429$ & 3391 & 5722 & - & - & 281 & $<2.6$ \\
\hline $966^{*}$ & $6: 41: 01.7$ & $9: 33: 00.1$ & 1.00 & 186 & - & - & - & - & - & - & 1.6 \\
\hline 967 & $6: 41: 01.8$ & $9: 34: 40.5$ & 1.00 & - & - & - & - & - & - & - & $<1.7$ \\
\hline 968 & $6: 41: 01.8$ & $9: 36: 30.2$ & 0.50 & - & $06410175+0936301$ & - & - & - & - & - & $<0.7$ \\
\hline 969 & $6: 41: 01.8$ & $9: 33: 34.7$ & 1.00 & - & - & - & - & - & - & - & $<0.6$ \\
\hline 970 & $6: 41: 01.8$ & $9: 36: 15.7$ & 0.67 & - & $06410182+0936156$ & - & - & - & - & - & $<1.0$ \\
\hline 971 & $6: 41: 01.8$ & $9: 34: 34.2$ & 0.50 & - & $06410182+0934342$ & - & - & - & - & - & $<1.6$ \\
\hline 972 & $6: 41: 01.8$ & $9: 37: 13.9$ & 0.50 & - & $06410183+0937139$ & - & 5726 & - & - & - & $<0.8$ \\
\hline $973 *$ & $6: 41: 01.8$ & 9:38:41.1 & 0.50 & 187 & $06410183+0938411$ & 3395 & 5727 & 82 & 146 & 283 & 38.8 \\
\hline 974 & $6: 41: 01.9$ & $9: 36: 05.2$ & 0.64 & - & $06410189+0936052$ & - & 5730 & - & - & - & $<1.2$ \\
\hline 975 & $6: 41: 01.9$ & $9: 26: 22.7$ & 0.69 & - & $06410194+0926227$ & - & 5733 & - & - & - & $<2.3$ \\
\hline 976 & $6: 41: 02.0$ & $9: 34: 30.8$ & 1.00 & - & - & - & - & - & - & - & $<1.5$ \\
\hline 977 & $6: 41: 02.0$ & $9: 41: 34.8$ & 0.50 & - & $06410200+0941348$ & - & 5734 & - & - & - & $<2.0$ \\
\hline 978 & $6: 41: 02.0$ & $9: 26: 23.4$ & 1.00 & - & - & - & 5736 & - & - & - & $<2.3$ \\
\hline $979 *$ & $6: 41: 02.0$ & $9: 38: 33.4$ & 0.50 & 188 & $06410202+0938333$ & - & 5737 & - & - & 285 & 3.3 \\
\hline 980 & $6: 41: 02.2$ & $9: 34: 14.6$ & 1.00 & - & - & - & - & - & - & - & $<1.1$ \\
\hline 981 & $6: 41: 02.2$ & $9: 34: 30.1$ & 1.00 & - & - & - & - & - & - & - & $<1.1$ \\
\hline $982 *$ & $6: 41: 02.3$ & $9: 27: 23.9$ & 0.50 & 189 & $06410230+0927238$ & - & 5750 & $87 \dagger$ & - & - & 22.9 \\
\hline 983 & $6: 41: 02.4$ & 9:39:00.9 & 0.52 & - & $06410241+0939008$ & - & - & - & - & - & $<1.1$ \\
\hline $984 *$ & $6: 41: 02.5$ & $9: 34: 55.8$ & 0.50 & 190 & $06410253+0934557$ & 3411 & 5757 & 85 & 149 & - & 71.2 \\
\hline 985 & $6: 41: 02.5$ & $9: 35: 02.1$ & 1.00 & - & - & - & - & - & - & - & $<0.8$ \\
\hline 986 & $6: 41: 02.6$ & $9: 34: 42.7$ & 1.00 & - & - & - & - & - & - & - & $<0.7$ \\
\hline $987 *$ & $6: 41: 02.6$ & $9: 35: 13.1$ & 0.50 & 193 & $06410258+0935131$ & 3412 & 5762 & 86 & 150 & 287 & 122.8 \\
\hline 988 & $6: 41: 02.6$ & $9: 34: 19.1$ & 0.50 & - & $06410258+0934190$ & 3413 & 5761 & - & 148 & - & $<1.4$ \\
\hline $989 *$ & $6: 41: 02.6$ & $9: 36: 15.6$ & 0.50 & 192 & $06410258+0936156$ & - & 5759 & - & - & - & 32.9 \\
\hline $990 *$ & $6: 41: 02.6$ & $9: 36: 40.2$ & 0.50 & 191 & $06410259+0936402$ & 3414 & 5760 & - & - & - & 4.8 \\
\hline 991 & $6: 41: 02.7$ & $9: 40: 45.3$ & 0.50 & - & $06410268+0940453$ & - & 5766 & - & - & - & $<1.7$ \\
\hline $992 *$ & $6: 41: 02.8$ & $9: 36: 16.2$ & 0.50 & 194 & $06410281+0936161$ & - & 5771 & - & - & - & 42.9 \\
\hline 993 & 6:41:02.8 & 9:34:08.1 & 1.00 & - & - & - & - & - & - & - & $<0.8$ \\
\hline $994 *$ & $6: 41: 02.8$ & $9: 35: 34.3$ & 0.50 & 195 & $06410281+0935343$ & - & - & - & - & - & 1.4 \\
\hline 995 & $6: 41: 02.9$ & $9: 36: 21.4$ & 0.50 & - & $06410288+0936214$ & - & - & - & - & - & $<2.0$ \\
\hline $996^{*}$ & $6: 41: 02.9$ & $9: 27: 23.5$ & 0.50 & 196 & $06410288+0927234$ & - & - & $87 \dagger$ & - & - & 9.0 \\
\hline $997 *$ & $6: 41: 03.0$ & $9: 37: 35.8$ & 0.50 & 197 & $06410303+0937357$ & 3428 & 5778 & - & - & 294 & 10.1 \\
\hline 998 & $6: 41: 03.1$ & $9: 31: 15.4$ & 0.50 & - & $06410305+0931154$ & - & 5779 & $89 \dagger$ & - & 295 & $<0.8$ \\
\hline 999 & $6: 41: 03.1$ & $9: 33: 27.9$ & 1.00 & - & - & - & 5781 & - & - & - & $<0.7$ \\
\hline 1000 & $6: 41: 03.1$ & $9: 34: 03.1$ & 1.00 & - & - & - & - & - & - & - & $<0.6$ \\
\hline 1001 & $6: 41: 03.1$ & $9: 28: 48.8$ & 0.50 & - & $06410307+0928488$ & - & - & - & - & - & $<1.4$ \\
\hline $1002 *$ & 6:41:03.1 & 9:29:04.6 & 0.50 & - & $06410308+0929045$ & 3430 & 5783 & - & - & 296 & $<1.3$ \\
\hline $1003 *$ & 6:41:03.1 & $9: 30: 55.7$ & 1.48 & 198 & - & - & - & - & - & - & 0.8 \\
\hline $1004 *$ & $6: 41: 03.1$ & $9: 35: 45.1$ & 0.50 & 199 & $06410313+0935450$ & - & - & - & - & - & 22.6 \\
\hline $1005^{*}$ & $6: 41: 03.2$ & $9: 26: 03.0$ & 0.50 & 200 & $06410319+0926030$ & - & 5785 & - & - & 297 & 17.8 \\
\hline $1006 *$ & $6: 41: 03.2$ & $9: 32: 55.0$ & 0.50 & 201 & $06410319+0932550$ & - & 5786 & - & - & - & 2.4 \\
\hline 1007 & $6: 41: 03.2$ & $9: 42: 45.8$ & 0.50 & - & $06410324+0942457$ & - & - & - & - & - & $<3.2$ \\
\hline 1008 & $6: 41: 03.3$ & $9: 39: 18.7$ & 0.50 & - & $06410329+0939186$ & - & 5787 & - & - & - & $<1.2$ \\
\hline $1009 *$ & 6:41:03.4 & 9:36:04.9 & 0.50 & 202 & $06410337+0936048$ & 3439 & 5792 & - & - & 300 & 11.6 \\
\hline $1010 *$ & 6:41:03.4 & $9: 40: 44.8$ & 0.50 & 203 & $06410338+0940448$ & 3438 & 5791 & - & 153 & 299 & 14.0 \\
\hline 1011 & $6: 41: 03.4$ & $9: 26: 37.1$ & 0.50 & - & $06410340+0926371$ & - & 5793 & - & - & - & $<2.2$ \\
\hline $1012 *$ & $6: 41: 03.4$ & 9:30:04.9 & 0.50 & 204 & $06410342+0930049$ & 3440 & 5796 & - & - & 301 & 27.1 \\
\hline 1013 & $6: 41: 03.4$ & $9: 42: 07.5$ & 0.50 & - & $06410343+0942074$ & - & - & - & - & - & $<2.2$ \\
\hline $1014 *$ & $6: 41: 03.4$ & $9: 32: 11.7$ & 0.50 & 205 & $06410344+0932117$ & 3442 & 5798 & - & - & - & 7.2 \\
\hline $1015 *$ & $6: 41: 03.5$ & $9: 31: 18.4$ & 0.50 & 206 & $06410349+0931184$ & 3443 & 5803 & $89 \dagger$ & 154 & - & 213.7 \\
\hline 1016 & $6: 41: 03.5$ & $9: 35: 24.8$ & 0.55 & - & $06410350+0935248$ & - & 5802 & - & - & - & $<0.7$ \\
\hline 1017 & $6: 41: 03.5$ & $9: 41: 05.5$ & 0.50 & - & $06410353+0941055$ & - & 5805 & - & - & - & $<1.8$ \\
\hline 1018 & $6: 41: 03.5$ & $9: 27: 20.8$ & 0.50 & - & $06410354+0927207$ & - & 5806 & - & - & - & $<2.9$ \\
\hline 1019 & $6: 41: 03.6$ & $9: 37: 45.4$ & 0.50 & - & $06410356+0937454$ & - & 5808 & - & - & - & $<2.0$ \\
\hline 1020 & 6:41:03.6 & $9: 34: 53.1$ & 1.00 & - & - & - & - & - & - & - & $<0.6$ \\
\hline
\end{tabular}


Table 3. (Continued)

\begin{tabular}{|c|c|c|c|c|c|c|c|c|c|c|c|}
\hline$\overline{\mathrm{N}}$ & $\begin{array}{c}\mathrm{RA} \\
{[\mathrm{h} \mathrm{m} \mathrm{s}]}\end{array}$ & $\begin{array}{c}\text { Dec. } \\
{[\mathrm{d} \mathrm{m} \mathrm{s}]}\end{array}$ & $\begin{array}{c}\text { Id. rad. } \\
\text { ["] } \\
\end{array}$ & $\overline{\mathrm{ACIS}}$ & 2Mass & Reb.+ & $\overline{\text { Lamm+ }}$ & Flacc+ & $\overline{\text { W56 }}$ & $\overline{\text { Dahm+ }}$ & $\begin{array}{l}\text { Ct. Rate. } \\
{\left[10^{-4} s^{-1}\right]}\end{array}$ \\
\hline 1021 & 6:41:03.6 & $9: 34: 04.5$ & $\overline{1.00}$ & $\overline{-1}$ & $\overline{-1}$ & $\overline{-1}$ & $\overline{-1}$ & $\overline{-}$ & $\overline{-}$ & $\overline{-1}$ & $\overline{<<0.8}$ \\
\hline $1022 *$ & 6:41:03.6 & 9:30:29.0 & 0.50 & 207 & $06410360+0930290$ & 3447 & 5810 & - & - & 302 & 17.5 \\
\hline $1023^{*}$ & 6:41:03.6 & 9:36:04.6 & 0.50 & 208 & $06410362+0936046$ & - & 5811 & - & - & - & 4.2 \\
\hline $1024 *$ & 6:41:03.6 & $9: 35: 43.9$ & 0.50 & 209 & $06410364+0935438$ & - & - & - & - & - & 1.4 \\
\hline 1025 & $6: 41: 03.7$ & $9: 27: 26.9$ & 1.00 & - & - & - & 5813 & - & - & - & $<2.9$ \\
\hline $1026 *$ & $6: 41: 03.7$ & $9: 28: 20.6$ & 1.00 & - & - & - & 5814 & - & - & - & $<3.0$ \\
\hline $1027 *$ & $6: 41: 03.7$ & $9: 28: 20.0$ & 0.50 & 210 & $06410370+0928200$ & 3450 & 5816 & - & - & 303 & 4.9 \\
\hline $1028 *$ & 6:41:03.7 & $9: 27: 24.4$ & 0.50 & 211 & $06410372+0927244$ & - & 5815 & - & - & - & 3.4 \\
\hline $1029 *$ & 6:41:03.7 & $9: 27: 39.8$ & 0.50 & 212 & $06410374+0927397$ & - & 5817 & 90 & - & 304 & 24.6 \\
\hline 1030 & 6:41:03.7 & 9:36:01.2 & 0.50 & - & $06410374+0936011$ & - & 5818 & - & - & - & $<0.8$ \\
\hline 1031 & $6: 41: 03.8$ & $9: 34: 54.3$ & 1.00 & - & - & - & 5819 & - & - & - & $<0.6$ \\
\hline 1032 & 6:41:03.8 & 9:33:23.1 & 1.00 & - & - & - & - & - & - & - & $<0.7$ \\
\hline 1033 & 6:41:03.9 & 9:35:03.9 & 1.00 & - & - & - & - & - & - & - & $<0.6$ \\
\hline 1034 & 6:41:03.9 & $9: 34: 33.0$ & 1.00 & - & - & - & - & - & - & - & $<0.6$ \\
\hline $1035^{*}$ & 6:41:03.9 & $9: 37: 11.1$ & 0.50 & 213 & $06410393+0937110$ & 3454 & 5824 & - & - & - & 10.2 \\
\hline 1036 & 6:41:04.0 & $9: 30: 19.5$ & 8.10 & - & - & - & - & 92 & - & - & $<1.0$ \\
\hline 1037 & 6:41:04.0 & $9: 35: 03.2$ & 1.00 & - & - & - & - & - & - & - & $<0.6$ \\
\hline 1038 & 6:41:04.0 & $9: 33: 18.1$ & 1.00 & - & - & - & - & - & - & - & $<0.7$ \\
\hline $1039 *$ & 6:41:04.0 & $9: 28: 24.4$ & 0.50 & 214 & $06410400+0928243$ & - & 5826 & - & - & - & 5.8 \\
\hline 1040 & 6:41:04.0 & $9: 35: 38.3$ & 0.50 & - & $06410401+0935383$ & - & - & - & - & - & $<0.7$ \\
\hline $1041 *$ & 6:41:04.1 & 9:33:48.6 & 0.50 & 215 & $06410405+0933486$ & - & 5831 & - & - & - & 3.0 \\
\hline $1042 *$ & 6:41:04.1 & $9: 35: 21.1$ & 0.50 & 216 & $06410406+0935211$ & 3461 & 5832 & 93 & - & 308 & 28.1 \\
\hline 1043 & 6:41:04.1 & $9: 40: 44.2$ & 0.50 & - & $06410406+0940441$ & - & - & - & - & - & $<1.8$ \\
\hline 1044 & 6:41:04.1 & 9:33:01.9 & 0.50 & - & $06410411+0933018$ & - & 5834 & - & 157 & - & $<0.7$ \\
\hline 1045 & 6:41:04.2 & $9: 26: 24.7$ & 0.50 & - & $06410416+0926247$ & - & - & - & - & - & $<2.5$ \\
\hline $1046^{*}$ & 6:41:04.2 & $9: 34: 57.2$ & 0.50 & 217 & $06410417+0934572$ & 3463 & 5837 & - & - & - & 4.8 \\
\hline $1047 *$ & 6:41:04.2 & $9: 36: 55.6$ & 0.50 & 219 & $06410419+0936556$ & 3465 & 5841 & - & - & - & 2.1 \\
\hline $1048 *$ & 6:41:04.2 & $9: 28: 40.5$ & 0.50 & 218 & $06410420+0928404$ & - & 5840 & - & - & - & 4.4 \\
\hline 1049 & 6:41:04.2 & $9: 33: 37.2$ & 1.00 & - & - & - & - & - & - & - & $<0.7$ \\
\hline 1050 & $6: 41: 04.2$ & $9: 33: 23.9$ & 1.00 & - & - & - & - & - & - & - & $<0.7$ \\
\hline 1051 & 6:41:04.2 & $9: 33: 32.1$ & 1.00 & - & - & - & - & - & - & - & $<0.7$ \\
\hline 1052 & 6:41:04.3 & 9:34:59.6 & 1.00 & - & - & - & - & - & - & - & $<0.6$ \\
\hline 1053 & 6:41:04.3 & $9: 34: 52.1$ & 1.00 & - & - & - & - & - & - & - & $<0.6$ \\
\hline 1054 & 6:41:04.3 & $9: 34: 45.6$ & 1.00 & - & - & - & - & - & - & - & $<0.6$ \\
\hline $1055^{*}$ & 6:41:04.3 & $9: 35: 33.3$ & 0.50 & 220 & $06410430+0935333$ & - & 5845 & - & - & 312 & 0.8 \\
\hline 1056 & 6:41:04.3 & $9: 40: 59.9$ & 0.50 & - & $06410430+0940599$ & - & 5846 & - & - & - & $<1.9$ \\
\hline 1057 & $6: 41: 04.3$ & 9:34:00.9 & 1.00 & - & - & - & - & - & - & - & $<0.7$ \\
\hline $1058^{*}$ & 6:41:04.3 & $9: 35: 47.8$ & 0.50 & 221 & $06410431+0935477$ & 3467 & 5847 & - & - & - & 4.8 \\
\hline $1059 *$ & 6:41:04.3 & $9: 34: 56.2$ & 1.00 & 222 & - & - & - & - & - & - & 0.8 \\
\hline 1060 & $6: 41: 04.4$ & $9: 32: 57.6$ & 0.50 & 224 & $06410436+0932576$ & - & 5850 & - & - & - & 1.1 \\
\hline $1061 *$ & $6: 41: 04.4$ & $9: 27: 30.7$ & 0.50 & 223 & $06410436+0927306$ & - & 5851 & - & - & - & 3.4 \\
\hline 1062 & 6:41:04.4 & $9: 33: 59.5$ & 1.00 & - & - & - & - & - & - & - & $<0.6$ \\
\hline 1063 & 6:41:04.4 & $9: 36: 22.8$ & 0.50 & - & $06410437+0936228$ & - & - & $96 \dagger$ & - & - & $<0.8$ \\
\hline $1064 *$ & 6:41:04.4 & $9: 36: 43.4$ & 0.50 & 225 & $06410444+0936433$ & - & 5854 & - & - & 313 & 1.6 \\
\hline 1065 & $6: 41: 04.5$ & $9: 33: 43.7$ & 1.00 & - & - & - & - & - & - & - & $<0.7$ \\
\hline 1066 & $6: 41: 04.5$ & $9: 34: 50.6$ & 1.00 & - & - & - & - & - & - & - & $<0.6$ \\
\hline 1067 & $6: 41: 04.5$ & $9: 36: 21.1$ & 1.00 & - & - & - & - & - & - & - & $<0.8$ \\
\hline $1068^{*}$ & $6: 41: 04.5$ & 9:30:13.4 & 0.50 & 226 & $06410450+0930134$ & 3474 & 5857 & - & - & 316 & 12.4 \\
\hline $1069 *$ & $6: 41: 04.5$ & $9: 26: 09.2$ & 0.50 & 227 & $06410454+0926092$ & 3475 & 5859 & - & - & 317 & 12.4 \\
\hline $1070 *$ & 6:41:04.6 & 9:38:30.9 & 0.50 & - & $06410457+0938308$ & 3476 & 5861 & - & - & 318 & $<2.7$ \\
\hline 1071 & 6:41:04.6 & $9: 34: 47.7$ & 1.00 & - & - & - & - & - & - & - & $<0.6$ \\
\hline $1072 *$ & 6:41:04.6 & $9: 36: 18.1$ & 1.00 & 228 & - & - & - & - & - & - & 28.7 \\
\hline 1073 & 6:41:04.6 & $9: 33: 54.5$ & 1.00 & - & - & - & - & - & - & - & $<0.6$ \\
\hline 1074 & 6:41:04.6 & 9:34:58.2 & 1.00 & - & - & - & - & - & - & - & $<0.7$ \\
\hline 1075 & $6: 41: 04.7$ & $9: 33: 48.7$ & 1.00 & - & - & - & - & - & - & - & $<0.7$ \\
\hline 1076 & 6:41:04.7 & $9: 34: 54.9$ & 1.00 & - & - & - & - & - & - & - & $<0.7$ \\
\hline $1077 *$ & 6:41:04.7 & $9: 36: 26.7$ & 0.50 & 229 & $06410471+0936267$ & - & 5865 & $96 \dagger$ & 159 & - & 49.2 \\
\hline 1078 & 6:41:04.8 & $9: 38: 23.9$ & 0.64 & - & $06410476+0938239$ & - & 5866 & - & - & - & $<2.4$ \\
\hline 1079 & 6:41:04.8 & 9:34:43.4 & 1.00 & - & - & - & - & - & - & - & $<0.6$ \\
\hline 1080 & 6:41:04.9 & 9:35:03.6 & 1.00 & - & - & - & - & - & - & - & $<0.7$ \\
\hline
\end{tabular}


Table 3. (Continued)

\begin{tabular}{|c|c|c|c|c|c|c|c|c|c|c|c|}
\hline$\overline{\mathrm{N}}$ & $\begin{array}{c}\mathrm{RA} \\
{[\mathrm{h} \mathrm{m} \mathrm{s}]}\end{array}$ & $\begin{array}{c}\text { Dec. } \\
{[\mathrm{d} \mathrm{m} \mathrm{s}]}\end{array}$ & $\begin{array}{c}\text { Id. rad. } \\
\text { ["] }\end{array}$ & ACIS & 2Mass & Reb.+ & $\overline{\text { Lamm+ }}$ & Flacc+ & $\overline{\text { W56 }}$ & $\overline{\mathrm{Dahm+}}$ & $\begin{array}{l}\text { Ct. Rate. } \\
{\left[10^{-4} s^{-1}\right]}\end{array}$ \\
\hline 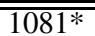 & 6 6:41:05.0 & $9: 42: 15.4$ & $\overline{2.44}$ & $\overline{230}$ & $\overline{-1}$ & $\overline{-1}$ & $\overline{-1}$ & $\overline{-1}$ & $\overline{-1}$ & 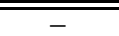 & $\overline{\overline{3.3}}$ \\
\hline 1082 & $6: 41: 05.0$ & 9:29:39.1 & 1.00 & - & - & - & 5875 & - & - & - & $<1.2$ \\
\hline 1083 & $6: 41: 05.0$ & $9: 38: 56.8$ & 0.54 & - & $06410504+0938567$ & - & - & - & - & - & $<3.0$ \\
\hline $1084 *$ & 6:41:05.1 & 9:33:00.3 & 0.50 & 231 & $06410505+0933002$ & - & 5876 & - & - & 324 & 20.9 \\
\hline 1085 & $6: 41: 05.2$ & $9: 34: 10.3$ & 1.00 & - & - & - & - & - & - & - & $<1.6$ \\
\hline $1086^{*}$ & $6: 41: 05.2$ & $9: 36: 31.5$ & 1.01 & 232 & - & - & - & - & - & - & 1.1 \\
\hline $1087 *$ & $6: 41: 05.2$ & 9:38:08.8 & 1.78 & 233 & - & - & - & - & - & - & 1.6 \\
\hline 1088 & $6: 41: 05.2$ & $9: 35: 35.8$ & 0.50 & - & $06410522+0935358$ & - & - & - & - & - & $<0.7$ \\
\hline 1089 & $6: 41: 05.2$ & $9: 35: 46.1$ & 1.00 & - & - & - & 5886 & - & - & - & $<0.7$ \\
\hline 1090 & $6: 41: 05.2$ & $9: 40: 17.7$ & 1.00 & - & - & - & 5887 & - & - & - & $<2.5$ \\
\hline 1091 & $6: 41: 05.3$ & 9:31:01.0 & 1.00 & - & - & - & 5889 & - & - & - & $<0.9$ \\
\hline $1092 *$ & $6: 41: 05.3$ & $9: 28: 12.5$ & 0.50 & - & $06410533+0928124$ & 3497 & 5890 & - & - & 332 & $<1.7$ \\
\hline 1093 & $6: 41: 05.4$ & 9:34:13.3 & 0.50 & - & $06410535+0934133$ & - & - & - & - & - & $<1.5$ \\
\hline $1094 *$ & $6: 41: 05.4$ & 9:33:13.4 & 0.50 & 234 & $06410536+0933134$ & 3500 & 5894 & 98 & 160 & 333 & 301.3 \\
\hline $1095^{*}$ & 6:41:05.4 & $9: 36: 30.9$ & 0.50 & 235 & $06410538+0936309$ & - & - & - & - & - & 11.4 \\
\hline 1096 & $6: 41: 05.4$ & $9: 33: 23.5$ & 1.00 & - & - & - & - & - & - & - & $<0.7$ \\
\hline 1097 & $6: 41: 05.4$ & 9:34:09.5 & 0.50 & $237 \dagger$ & $06410542+0934095$ & 3502 & 5895 & - & - & - & $<2.2$ \\
\hline $1098 *$ & $6: 41: 05.5$ & $9: 36: 26.5$ & 1.00 & 236 & - & - & - & - & - & - & 1.8 \\
\hline 1099 & $6: 41: 05.5$ & 9:34:11.7 & 1.00 & - & - & - & - & - & - & - & $<1.4$ \\
\hline 1100 & $6: 41: 05.5$ & $9: 34: 36.9$ & 1.00 & - & - & - & - & - & - & - & $<0.7$ \\
\hline 1101 & $6: 41: 05.5$ & 9:35:01.4 & 1.00 & - & - & - & - & - & - & - & $<0.7$ \\
\hline $1102 *$ & $6: 41: 05.5$ & $9: 31: 40.5$ & 0.50 & 238 & $06410554+0931405$ & - & 5897 & $99 \dagger$ & - & 335 & 24.6 \\
\hline 1103 & 6:41:05.6 & 9:34:08.1 & 1.00 & - & - & - & - & - & - & - & $<2.0$ \\
\hline 1104 & $6: 41: 05.6$ & 9:34:05.9 & 0.50 & $237 \dagger$ & $06410561+0934058$ & - & - & - & - & - & $<2.2$ \\
\hline 1105 & 6:41:05.6 & $9: 33: 54.9$ & 0.50 & - & $06410562+0933549$ & - & 5898 & - & - & - & $<0.7$ \\
\hline $1106 *$ & $6: 41: 05.6$ & $9: 35: 44.5$ & 0.50 & 239 & $06410563+0935445$ & - & - & - & - & - & 3.1 \\
\hline 1107 & $6: 41: 05.7$ & $9: 37: 43.0$ & 0.50 & - & $06410571+0937430$ & - & - & - & - & - & $<0.9$ \\
\hline 1108 & 6:41:05.7 & $9: 34: 06.3$ & 1.00 & - & - & - & - & - & - & - & $<1.4$ \\
\hline $1109 *$ & $6: 41: 05.7$ & 9:31:01.3 & 0.50 & 240 & $06410574+0931012$ & 3514 & 5902 & $100 \dagger$ & 162 & 337 & 63.0 \\
\hline 1110 & $6: 41: 05.7$ & $9: 33: 48.3$ & 1.00 & - & - & - & 5901 & - & - & - & $<0.7$ \\
\hline 1111 & $6: 41: 05.8$ & $9: 33: 00.5$ & 1.00 & - & - & - & - & - & - & - & $<0.7$ \\
\hline $1112 *$ & $6: 41: 05.8$ & $9: 36: 32.0$ & 0.50 & 241 & $06410576+0936319$ & - & 5903 & - & - & - & 5.3 \\
\hline $1113 *$ & $6: 41: 05.8$ & $9: 35: 29.6$ & 0.50 & 242 & $06410577+0935295$ & - & - & - & - & - & 18.8 \\
\hline $1114 *$ & $6: 41: 05.8$ & 9:31:07.3 & 0.50 & 243 & $06410579+0931072$ & - & 5906 & $100 \dagger$ & - & - & 2.2 \\
\hline 1115 & $6: 41: 05.9$ & $9: 34: 52.8$ & 1.00 & - & - & - & - & - & - & - & $<0.7$ \\
\hline $1116^{*}$ & $6: 41: 05.9$ & $9: 34: 45.9$ & 1.00 & 244 & - & - & - & - & - & - & 81.6 \\
\hline 1117 & 6:41:05.9 & $9: 41: 25.3$ & 0.52 & - & $06410590+0941253$ & - & 5913 & - & - & - & $<2.6$ \\
\hline 1118 & $6: 41: 05.9$ & 9:34:04.9 & 1.00 & - & - & - & - & - & - & - & $<1.4$ \\
\hline 1119 & $6: 41: 05.9$ & $9: 35: 03.2$ & 1.00 & - & - & - & - & - & - & - & $<0.7$ \\
\hline $1120 *$ & $6: 41: 05.9$ & $9: 27: 17.4$ & 0.50 & 245 & $06410593+0927174$ & 3519 & 5912 & - & - & 340 & 14.2 \\
\hline 1121 & $6: 41: 06.0$ & $9: 34: 39.8$ & 1.00 & - & - & - & - & - & - & - & $<0.7$ \\
\hline $1122 *$ & $6: 41: 06.0$ & $9: 31: 39.8$ & 0.50 & 246 & $06410596+0931398$ & - & 5914 & $99 \dagger$ & - & - & 19.0 \\
\hline $1123^{*}$ & $6: 41: 06.0$ & $9: 39: 14.2$ & 0.50 & - & $06410597+0939142$ & 3520 & 5915 & - & - & 341 & $<1.5$ \\
\hline 1124 & $6: 41: 06.0$ & $9: 34: 11.5$ & 0.50 & - & $06410598+0934115$ & - & - & - & - & - & $<1.5$ \\
\hline $1125 *$ & $6: 41: 06.0$ & $9: 35: 51.3$ & 0.50 & 247 & $06410599+0935513$ & 3522 & 5916 & - & - & - & 1.9 \\
\hline 1126 & $6: 41: 06.0$ & $9: 34: 46.2$ & 0.50 & 248 & 06410604+0934461 & - & 5920 & - & - & - & 8.5 \\
\hline 1127 & 6:41:06.0 & $9: 27: 08.5$ & 0.50 & - & $06410604+0927084$ & - & 5919 & - & - & - & $<2.6$ \\
\hline $1128 *$ & $6: 41: 06.1$ & $9: 29: 26.4$ & 0.50 & - & $06410614+0929263$ & - & 5922 & $103 \dagger$ & - & - & $<1.3$ \\
\hline 1129 & 6:41:06.2 & 9:34:08.9 & 1.00 & - & - & - & - & - & - & - & $<1.5$ \\
\hline $1130 *$ & 6:41:06.2 & $9: 36: 23.0$ & 0.50 & 249 & $06410620+0936229$ & 3525 & 5924 & 101 & 164 & 342 & 228.2 \\
\hline $1131 *$ & 6:41:06.2 & 9:33:08.8 & 0.50 & 250 & $06410623+0933087$ & 3527 & 5927 & - & - & 343 & 11.1 \\
\hline 1132 & $6: 41: 06.2$ & 9:40:09.1 & 1.00 & - & - & - & 5928 & - & - & - & $<3.0$ \\
\hline $1133 *$ & $6: 41: 06.3$ & $9: 29: 40.5$ & 0.50 & 251 & $06410626+0929404$ & - & 5929 & $103 \dagger$ & - & - & 3.3 \\
\hline 1134 & $6: 41: 06.3$ & 9:33:49.7 & 0.50 & - & $06410629+0933496$ & - & - & - & - & - & $<0.7$ \\
\hline $1135^{*}$ & 6:41:06.3 & $9: 29: 31.0$ & 1.00 & 252 & - & 3528 & 5932 & $103 \dagger$ & - & 345 & 13.3 \\
\hline 1136 & $6: 41: 06.3$ & $9: 40: 10.6$ & 0.50 & - & $06410634+0940105$ & 3531 & 5934 & - & - & 346 & $<2.7$ \\
\hline 1137 & $6: 41: 06.3$ & $9: 34: 15.3$ & 1.00 & - & - & - & - & - & - & - & $<1.4$ \\
\hline $1138 *$ & 6:41:06.4 & 9:37:08.6 & 0.50 & 253 & $06410637+0937085$ & - & - & - & - & - & 2.2 \\
\hline 1139 & $6: 41: 06.4$ & $9: 35: 06.1$ & 0.50 & - & $06410640+0935061$ & - & 5937 & - & - & - & $<0.7$ \\
\hline 1140 & 6:41:06.4 & $9: 30: 33.7$ & 1.00 & - & - & - & 5940 & - & - & - & $<1.0$ \\
\hline
\end{tabular}


Table 3. (Continued)

\begin{tabular}{|c|c|c|c|c|c|c|c|c|c|c|c|}
\hline$\overline{\mathrm{N}}$ & $\begin{array}{c}\mathrm{RA} \\
{[\mathrm{h} \mathrm{m} \mathrm{s}]}\end{array}$ & $\begin{array}{c}\text { Dec. } \\
{[\mathrm{d} \mathrm{m} \mathrm{s}]}\end{array}$ & $\begin{array}{c}\text { Id. rad. } \\
\text { ["] } \\
\end{array}$ & $\overline{\mathrm{ACIS}}$ & 2Mass & Reb.+ & $\overline{\text { Lamm+ }}$ & Flacc+ & $\overline{\text { W56 }}$ & $\overline{\text { Dahm+ }}$ & $\begin{array}{l}\text { Ct. Rate. } \\
{\left[10^{-4} s^{-1}\right]}\end{array}$ \\
\hline 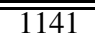 & $\overline{76: 41: 06.4}$ & 9:34:10.0 & $\overline{0.50}$ & $\overline{-1}$ & $06410642+0934099$ & $\overline{-1}$ & $\overline{-1}$ & - & - & $\overline{-1}$ & $\overline{<<1.5}$ \\
\hline $1142 *$ & 6:41:06.4 & $9: 28: 38.8$ & 0.50 & 254 & $06410642+0928388$ & 3534 & 5941 & - & - & 348 & 9.7 \\
\hline $1143 *$ & 6:41:06.4 & $9: 26: 58.7$ & 0.50 & 255 & $06410643+0926587$ & - & 5939 & - & - & - & 153.9 \\
\hline 1144 & 6:41:06.4 & $9: 33: 43.3$ & 1.00 & - & - & - & - & - & - & - & $<0.7$ \\
\hline $1145^{*}$ & $6: 41: 06.4$ & $9: 38: 37.1$ & 0.50 & 256 & 06410644+0938371 & - & - & - & - & - & 2.5 \\
\hline $1146^{*}$ & $6: 41: 06.5$ & $9: 36: 05.5$ & 0.50 & 257 & $06410647+0936055$ & - & - & - & - & - & 39.3 \\
\hline 1147 & $6: 41: 06.5$ & $9: 35: 54.1$ & 1.00 & - & - & - & - & - & - & - & $<0.8$ \\
\hline 1148 & $6: 41: 06.5$ & 9:30:05.1 & 1.00 & - & - & - & 5944 & - & - & - & $<1.2$ \\
\hline 1149 & 6:41:06.6 & $9: 35: 45.2$ & 0.50 & 258 & $06410659+0935451$ & 3538 & 5945 & - & - & - & 2.0 \\
\hline 1150 & 6:41:06.6 & $9: 35: 00.3$ & 1.00 & - & - & - & - & - & - & - & $<0.7$ \\
\hline 1151 & 6:41:06.6 & $9: 35: 02.1$ & 1.00 & - & - & - & - & - & - & - & $<0.7$ \\
\hline 1152 & 6:41:06.6 & 9:34:21.1 & 1.00 & - & - & - & - & - & - & - & $<0.9$ \\
\hline 1153 & $6: 41: 06.7$ & 9:33:57.7 & 0.50 & - & $06410665+0933576$ & - & - & - & - & - & $<1.0$ \\
\hline 1154 & 6:41:06.7 & $9: 34: 26.5$ & 1.00 & - & - & - & - & - & - & - & $<0.7$ \\
\hline 1155 & 6:41:06.7 & $9: 33: 30.0$ & 1.00 & - & - & - & - & - & - & - & $<0.7$ \\
\hline 1156 & $6: 41: 06.7$ & $9: 41: 46.6$ & 1.00 & - & - & - & 5947 & - & - & - & $<4.2$ \\
\hline $1157 *$ & 6:41:06.7 & $9: 33: 54.3$ & 1.00 & 259 & - & - & - & - & - & - & 0.7 \\
\hline 1158 & 6:41:06.7 & 9:34:38.0 & 1.00 & - & - & - & - & - & - & - & $<0.7$ \\
\hline 1159 & 6:41:06.7 & $9: 34: 31.9$ & 1.00 & - & - & - & - & - & - & - & $<0.7$ \\
\hline $1160 *$ & 6:41:06.7 & $9: 34: 45.9$ & 0.50 & 260 & $06410673+0934459$ & 3541 & 5949 & - & 166 & 349 & 7.8 \\
\hline 1161 & 6:41:06.7 & $9: 34: 59.3$ & 1.00 & - & - & - & - & - & - & - & $<0.7$ \\
\hline 1162 & 6:41:06.7 & 9:34:53.9 & 1.00 & - & - & - & - & - & - & - & $<0.7$ \\
\hline 1163 & 6:41:06.8 & $9: 33: 34.7$ & 1.00 & - & - & - & - & - & - & - & $<0.7$ \\
\hline $1164 *$ & 6:41:06.8 & $9: 27: 32.3$ & 0.50 & 261 & $06410682+0927322$ & 3543 & 5952 & $104 \dagger$ & - & 351 & 168.7 \\
\hline 1165 & 6:41:06.8 & $9: 35: 02.5$ & 1.00 & - & - & - & - & - & - & - & $<0.7$ \\
\hline 1166 & 6:41:06.9 & $9: 41: 46.1$ & 0.50 & - & $06410687+0941460$ & 3544 & 5954 & - & - & - & $<4.5$ \\
\hline $1167 *$ & $6: 41: 06.9$ & $9: 29: 24.0$ & 0.50 & 263 & $06410689+0929240$ & 3546 & 5956 & - & - & 352 & 8.7 \\
\hline 1168 & 6:41:07.0 & $9: 35: 55.8$ & 0.50 & - & $06410696+0935557$ & - & 5957 & - & - & - & $<0.8$ \\
\hline $1169 *$ & 6:41:07.0 & $9: 40: 54.5$ & 0.50 & 262 & $06410696+0940544$ & 3548 & 5958 & - & - & 353 & 3.1 \\
\hline 1170 & 6:41:07.1 & $9: 38: 11.2$ & 0.50 & - & $06410705+0938112$ & - & 5961 & - & - & - & $<1.1$ \\
\hline 1171 & $6: 41: 07.1$ & $9: 30: 37.5$ & 0.50 & - & $06410706+0930375$ & - & 5960 & - & - & - & $<3.4$ \\
\hline 1172 & 6:41:07.1 & $9: 38: 23.6$ & 0.74 & - & 06410706+0938235 & - & 5962 & - & - & - & $<1.1$ \\
\hline 1173 & 6:41:07.1 & $9: 34: 42.3$ & 1.00 & - & - & - & - & - & - & - & $<0.7$ \\
\hline 1174 & 6:41:07.1 & $9: 27: 09.8$ & 1.00 & - & - & - & 5965 & - & - & - & $<2.7$ \\
\hline 1175 & 6:41:07.1 & 9:34:44.9 & 1.00 & - & - & - & - & - & - & - & $<0.7$ \\
\hline $1176^{*}$ & 6:41:07.1 & $9: 25: 54.9$ & 1.00 & - & - & - & 5966 & - & - & 356 & $<42.2$ \\
\hline $1177 *$ & 6:41:07.2 & $9: 30: 36.7$ & 1.00 & 264 & - & - & - & - & - & - & 5.2 \\
\hline $1178 *$ & 6:41:07.2 & $9: 27: 48.0$ & 0.50 & 265 & $06410715+0927479$ & 3552 & 5968 & 105 & - & 355 & 67.2 \\
\hline $1179 *$ & 6:41:07.2 & $9: 27: 29.4$ & 0.50 & - & $06410715+0927294$ & - & 5967 & $104 \dagger$ & - & 354 & $<132.5$ \\
\hline 1180 & $6: 41: 07.2$ & $9: 34: 24.3$ & 0.50 & - & $06410719+0934242$ & - & - & - & - & - & $<0.7$ \\
\hline $1181 *$ & 6:41:07.2 & $9: 42: 25.6$ & 0.50 & 266 & $06410721+0942256$ & 3554 & 5969 & - & - & - & 6.2 \\
\hline 1182 & $6: 41: 07.2$ & $9: 34: 41.3$ & 1.00 & - & - & - & - & - & - & - & $<0.7$ \\
\hline 1183 & 6:41:07.2 & $9: 36: 59.3$ & 1.00 & 267 & - & - & 5970 & - & - & - & 1.0 \\
\hline $1184 *$ & $6: 41: 07.3$ & $9: 31: 18.1$ & 0.50 & 268 & $06410729+0931180$ & 3556 & 5973 & 107 & - & - & 11.2 \\
\hline $1185^{*}$ & 6:41:07.3 & $9: 25: 55.0$ & 0.50 & 269 & $06410734+0925549$ & 3559 & 5974 & 106 & - & 359 & 53.2 \\
\hline 1186 & 6:41:07.3 & $9: 34: 40.5$ & 1.00 & - & - & - & - & - & - & - & $<0.7$ \\
\hline 1187 & 6:41:07.4 & $9: 35: 20.8$ & 0.50 & - & $06410739+0935207$ & - & 5975 & - & - & - & $<0.7$ \\
\hline $1188^{*}$ & 6:41:07.4 & 9:31:11.1 & 0.50 & 271 & $06410740+0931110$ & 3563 & 5976 & - & - & 360 & 5.0 \\
\hline $1189 *$ & 6:41:07.4 & $9: 34: 55.0$ & 0.50 & 270 & $06410740+0934549$ & - & - & - & - & - & 0.8 \\
\hline $1190 *$ & 6:41:07.4 & 9:34:33.6 & 0.50 & 272 & $06410744+0934335$ & 3565 & 5978 & - & - & 361 & 0.7 \\
\hline 1191 & 6:41:07.4 & 9:30:00.9 & 1.00 & - & - & - & 5979 & - & - & - & $<8.5$ \\
\hline $1192 *$ & $6: 41: 07.5$ & $9: 33: 36.9$ & 0.50 & - & $06410748+0933369$ & 3566 & 5980 & - & - & - & $<0.7$ \\
\hline $1193 *$ & 6:41:07.6 & $9: 41: 33.6$ & 0.50 & - & $06410755+0941335$ & 3568 & 5982 & - & - & 362 & $<4.2$ \\
\hline $1194 *$ & 6:41:07.6 & $9: 30: 00.5$ & 0.50 & 273 & $06410755+0930004$ & 3569 & 5983 & - & - & - & 9.9 \\
\hline 1195 & 6:41:07.6 & $9: 30: 29.2$ & 0.50 & - & $06410757+0930292$ & - & - & - & - & - & $<1.2$ \\
\hline $1196 *$ & $6: 41: 07.7$ & $9: 34: 19.2$ & 1.00 & 274 & - & - & - & - & - & - & 28.7 \\
\hline $1197 *$ & 6:41:07.7 & $9: 41: 26.4$ & 0.50 & - & $06410770+0941264$ & 3573 & 5985 & - & - & 363 & $<3.4$ \\
\hline 1198 & 6:41:07.7 & $9: 29: 13.7$ & 0.50 & - & $06410773+0929136$ & - & - & - & - & - & $<1.5$ \\
\hline 1199* & 6:41:07.8 & 9:41:14.9 & 0.50 & 275 & 06410776+0941149 & 3574 & 5987 & - & 170 & - & 9.0 \\
\hline $1200 *$ & 6:41:07.8 & $9: 28: 13.5$ & 0.50 & 276 & 06410776+0928134 & 3575 & 5986 & - & - & 364 & 41.8 \\
\hline
\end{tabular}


Table 3. (Continued)

\begin{tabular}{|c|c|c|c|c|c|c|c|c|c|c|c|}
\hline$\overline{\mathrm{N}}$ & $\begin{array}{c}\mathrm{RA} \\
{[\mathrm{h} \mathrm{m} \mathrm{s}]}\end{array}$ & $\begin{array}{c}\text { Dec. } \\
{[\mathrm{d} \mathrm{m} \mathrm{s}]}\end{array}$ & $\begin{array}{c}\text { Id. rad. } \\
\text { ["] }\end{array}$ & $\overline{\mathrm{ACIS}}$ & 2Mass & $\overline{\text { Reb.+ }}$ & $\overline{\text { Lamm+ }}$ & Flacc+ & $\overline{\text { W56 }}$ & $\overline{\mathrm{Dahm+}}$ & $\begin{array}{l}\text { Ct. Rate. } \\
{\left[10^{-4} s^{-1}\right]}\end{array}$ \\
\hline 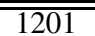 & 6 6:41:07.8 & $9: 41: 29.5$ & $\overline{0.50}$ & - & "06410781+0941294 & $\overline{-1}$ & $\overline{\overline{5991}}$ & - & $\overline{-1}$ & - & 安3.3 \\
\hline $1202 *$ & $6: 41: 07.8$ & $9: 26: 24.7$ & 0.50 & - & $06410781+0926247$ & 3579 & 5990 & - & - & - & $<2.5$ \\
\hline 1203 & $6: 41: 07.9$ & $9: 33: 33.9$ & 1.00 & - & - & - & - & - & - & - & $<0.7$ \\
\hline 1204 & 6:41:07.9 & $9: 34: 27.9$ & 1.00 & - & - & - & - & - & - & - & $<0.7$ \\
\hline 1205 & $6: 41: 07.9$ & 9:34:33.7 & 1.00 & - & - & - & - & - & - & - & $<0.7$ \\
\hline 1206 & $6: 41: 08.0$ & 9:34:16.4 & 1.00 & - & - & - & - & - & - & - & $<1.0$ \\
\hline $1207 *$ & $6: 41: 08.0$ & 9:34:47.0 & 0.50 & 277 & $06410798+0934469$ & 3580 & 5992 & - & - & - & 2.3 \\
\hline $1208 *$ & 6:41:08.0 & $9: 30: 40.3$ & 0.50 & 278 & $06410801+0930403$ & 3582 & 5994 & 109 & 175 & 365 & 25.8 \\
\hline 1209 & $6: 41: 08.0$ & $9: 27: 56.0$ & 1.00 & - & - & - & 5995 & - & - & - & $<2.3$ \\
\hline 1210 & 6:41:08.1 & $9: 33: 13.6$ & 1.00 & - & - & - & 5996 & - & - & - & $<0.7$ \\
\hline 1211 & $6: 41: 08.1$ & $9: 34: 30.1$ & 1.00 & - & - & - & - & - & - & - & $<0.7$ \\
\hline 1212 & $6: 41: 08.1$ & 9:34:16.1 & 1.00 & - & - & - & - & - & - & - & $<1.0$ \\
\hline $1213 *$ & $6: 41: 08.2$ & 9:30:03.9 & 0.50 & - & $06410817+0930038$ & - & 6000 & - & - & - & $<1.3$ \\
\hline $1214 *$ & $6: 41: 08.2$ & 9:30:08.0 & 0.50 & 279 & $06410818+0930079$ & - & - & - & - & - & 9.8 \\
\hline $1215 *$ & $6: 41: 08.2$ & $9: 36: 56.2$ & 0.50 & 280 & $06410820+0936561$ & 3588 & 6001 & - & - & - & 33.1 \\
\hline $1216^{*}$ & $6: 41: 08.2$ & $9: 34: 09.5$ & 0.50 & 281 & $06410821+0934094$ & 3591 & 6002 & - & - & 367 & 1.9 \\
\hline $1217 *$ & $6: 41: 08.2$ & 9:38:30.4 & 0.50 & 282 & $06410821+0938303$ & 3590 & 6003 & - & - & 366 & 46.9 \\
\hline $1218 *$ & $6: 41: 08.3$ & $9: 30: 22.8$ & 0.50 & - & $06410827+0930228$ & - & 6004 & - & - & 368 & $<1.2$ \\
\hline 1219 & 6:41:08.3 & 9:34:06.7 & 1.00 & - & - & - & - & - & - & - & $<1.7$ \\
\hline 1220 & $6: 41: 08.3$ & 9:38:14.3 & 0.50 & - & $06410828+0938143$ & 3592 & 6005 & - & 171 & - & $<1.1$ \\
\hline 1221 & $6: 41: 08.3$ & $9: 34: 25.8$ & 1.00 & - & - & - & - & - & - & - & $<0.7$ \\
\hline $1222 *$ & $6: 41: 08.3$ & $9: 29: 39.6$ & 0.50 & 283 & $06410833+0929395$ & - & - & - & - & - & 2.1 \\
\hline $1223 *$ & $6: 41: 08.4$ & $9: 40: 36.2$ & 0.50 & 284 & $06410837+0940361$ & 3594 & 6007 & - & - & 369 & 4.4 \\
\hline $1224 *$ & $6: 41: 08.4$ & $9: 37: 52.5$ & 0.50 & 285 & $06410843+0937525$ & 3595 & 6008 & - & - & 370 & 7.4 \\
\hline 1225 & 6:41:08.5 & $9: 25: 59.6$ & 0.50 & - & $06410849+0925595$ & - & - & - & - & - & $<3.1$ \\
\hline $1226 *$ & $6: 41: 08.5$ & $9: 31: 10.7$ & 1.00 & 286 & - & - & - & - & - & - & 1.4 \\
\hline 1227 & $6: 41: 08.5$ & $9: 32: 50.8$ & 0.50 & - & $06410854+0932508$ & - & 6010 & - & - & - & $<0.8$ \\
\hline $1228 *$ & $6: 41: 08.5$ & $9: 34: 13.2$ & 0.50 & 287 & $06410854+0934132$ & - & - & - & - & - & 9.4 \\
\hline 1229 & 6:41:08.6 & 9:31:05.9 & 0.50 & - & $06410857+0931058$ & - & 6012 & - & - & - & $<0.9$ \\
\hline 1230 & $6: 41: 08.6$ & 9:34:08.1 & 1.00 & - & - & - & - & - & - & - & $<1.6$ \\
\hline 1231 & $6: 41: 08.6$ & $9: 35: 42.1$ & 1.00 & - & - & - & - & - & - & - & $<0.7$ \\
\hline 1232 & 6:41:08.6 & $9: 28: 13.8$ & 1.00 & - & - & - & 6015 & - & - & - & $<2.0$ \\
\hline $1233 *$ & 6:41:08.6 & 9:30:03.6 & 0.50 & 288 & $06410859+0930036$ & - & 6014 & - & - & 372 & 1.4 \\
\hline 1234 & 6:41:08.6 & $9: 36: 03.4$ & 0.55 & - & $06410863+0936033$ & - & - & - & - & - & $<0.8$ \\
\hline $1235^{*}$ & $6: 41: 08.6$ & $9: 40: 13.3$ & 0.50 & - & $06410864+0940133$ & 3599 & 6019 & - & - & - & $<1.7$ \\
\hline 1236 & 6:41:08.7 & 9:39:11.4 & 1.00 & - & - & - & 6018 & - & - & - & $<1.3$ \\
\hline $1237 *$ & 6:41:08.7 & $9: 38: 25.3$ & 1.79 & 289 & - & - & - & - & - & - & 1.3 \\
\hline 1238 & 6:41:08.7 & $9: 29: 56.8$ & 0.50 & - & $06410871+0929567$ & - & - & - & - & - & $<1.4$ \\
\hline 1239 & $6: 41: 08.7$ & 9:31:13.9 & 0.76 & - & $06410872+0931139$ & - & - & - & - & - & $<0.9$ \\
\hline 1240 & $6: 41: 08.8$ & $9: 29: 51.7$ & 0.59 & - & $06410880+0929516$ & - & - & - & - & - & $<1.4$ \\
\hline 1241 & 6:41:08.8 & $9: 27: 53.3$ & 0.50 & - & $06410880+0927533$ & - & 6021 & - & - & - & $<2.4$ \\
\hline 1242 & $6: 41: 08.9$ & $9: 29: 55.1$ & 0.59 & - & $06410889+0929550$ & - & - & - & - & - & $<1.4$ \\
\hline 1243 & 6:41:08.9 & $9: 29: 45.1$ & 0.50 & - & $06410890+0929451$ & - & 6028 & - & - & - & $<1.5$ \\
\hline $1244 *$ & $6: 41: 08.9$ & $9: 41: 14.8$ & 0.50 & 290 & $06410891+0941147$ & 3608 & 6031 & 112 & 174 & - & 48.9 \\
\hline 1245 & 6:41:08.9 & $9: 29: 29.2$ & 0.50 & - & $06410892+0929291$ & - & 6029 & - & - & - & $<1.6$ \\
\hline 1246 & 6:41:08.9 & $9: 29: 56.8$ & 0.50 & - & $06410894+0929568$ & - & - & - & - & - & $<1.4$ \\
\hline $1247 *$ & 6:41:09.0 & $9: 33: 46.0$ & 0.50 & 291 & $06410896+0933460$ & 3611 & 6032 & - & - & 374 & 1.7 \\
\hline 1248 & 6:41:09.0 & $9: 29: 21.7$ & 0.50 & - & $06410896+0929217$ & - & 6030 & - & - & - & $<1.6$ \\
\hline $1249 *$ & 6:41:09.0 & 9:31:08.9 & 0.50 & - & $06410899+0931088$ & - & 6033 & - & - & 375 & $<0.9$ \\
\hline $1250 *$ & 6:41:09.1 & $9: 29: 07.2$ & 0.50 & 292 & $06410905+0929072$ & - & 6036 & - & - & - & 14.3 \\
\hline 1251 & 6:41:09.1 & $9: 29: 19.1$ & 0.75 & - & $06410907+0929190$ & - & - & - & - & - & $<1.6$ \\
\hline $1252 *$ & 6:41:09.1 & 9:30:09.1 & 0.50 & 293 & $06410908+0930090$ & - & 6039 & - & - & 376 & 7.4 \\
\hline 1253 & $6: 41: 09.1$ & $9: 29: 43.1$ & 1.00 & - & - & - & 6038 & - & - & - & $<1.5$ \\
\hline 1254 & $6: 41: 09.2$ & $9: 26: 40.2$ & 0.61 & - & $06410916+0926402$ & - & 6040 & - & - & - & $<2.5$ \\
\hline 1255 & 6:41:09.2 & $9: 26: 50.7$ & 0.50 & - & $06410922+0926507$ & - & 6044 & - & - & - & $<2.5$ \\
\hline 1256 & $6: 41: 09.2$ & $9: 29: 04.5$ & 1.00 & - & - & - & 6047 & - & - & - & $<6.9$ \\
\hline 1257 & 6:41:09.3 & $9: 30: 25.7$ & 0.50 & - & $06410928+0930257$ & - & - & - & - & - & $<1.1$ \\
\hline 1258 & $6: 41: 09.5$ & $9: 29: 59.6$ & 0.61 & - & $06410946+0929595$ & - & 6053 & - & - & - & $<1.3$ \\
\hline 1259 & $6: 41: 09.5$ & $9: 27: 45.9$ & 1.00 & - & - & - & 6056 & - & - & - & $<128.2$ \\
\hline $1260 *$ & 6:41:09.5 & $9: 35: 25.5$ & 0.50 & 294 & $06410951+0935254$ & 3620 & 6057 & - & - & - & 6.5 \\
\hline
\end{tabular}


Table 3. (Continued)

\begin{tabular}{|c|c|c|c|c|c|c|c|c|c|c|c|}
\hline$\overline{\mathrm{N}}$ & $\begin{array}{c}\mathrm{RA} \\
{[\mathrm{h} \mathrm{m} \mathrm{s}]}\end{array}$ & $\begin{array}{c}\text { Dec. } \\
{[\mathrm{d} \mathrm{m} \mathrm{s}]}\end{array}$ & $\begin{array}{c}\text { Id. rad. } \\
\text { ["] }\end{array}$ & $\overline{\mathrm{ACIS}}$ & 2Mass & Reb.+ & $\overline{\text { Lamm+ }}$ & Flacc+ & $\overline{\text { W56 }}$ & $\overline{\overline{\text { Dahm+ }}}$ & $\begin{array}{l}\text { Ct. Rate. } \\
{\left[10^{-4} s^{-1}\right]}\end{array}$ \\
\hline 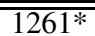 & $6: 6: 41: 09.5$ & 9 & $\overline{\overline{1.98}}$ & 295 & $\overline{-1}$ & - & $\overline{-1}$ & - & - & $\overline{-1}$ & $\overline{\overline{3.5}}$ \\
\hline $1262 *$ & $6: 41: 09.5$ & $9: 29: 25.1$ & 0.50 & 296 & $06410954+0929250$ & - & - & - & - & - & 49.7 \\
\hline $1263 *$ & 6:41:09.6 & $9: 27: 57.5$ & 0.50 & 297 & $06410959+0927574$ & - & - & 113 & - & - & 9.1 \\
\hline 1264 & 6:41:09.6 & $9: 29: 46.7$ & 0.54 & - & $06410960+0929466$ & - & - & - & - & - & $<1.5$ \\
\hline $1265^{*}$ & 6:41:09.6 & $9: 36: 29.1$ & 0.50 & 298 & $06410962+0936291$ & - & 6061 & - & - & - & 3.2 \\
\hline 1266 & 6:41:09.6 & $9: 29: 40.2$ & 0.50 & - & $06410964+0929402$ & - & - & - & - & - & $<3.1$ \\
\hline 1267 & 6:41:09.7 & $9: 29: 20.7$ & 0.50 & - & $06410965+0929207$ & - & - & - & - & - & $<1.7$ \\
\hline 1268 & 6:41:09.8 & $9: 29: 36.7$ & 0.54 & - & $06410976+0929366$ & - & - & - & - & - & $<3.1$ \\
\hline $1269 *$ & 6:41:09.8 & $9: 29: 15.4$ & 1.25 & 299 & - & - & - & - & - & - & 2.6 \\
\hline $1270 *$ & 6:41:09.8 & $9: 27: 12.2$ & 0.50 & 300 & $06410982+0927122$ & 3625 & 6067 & 114 & - & 379 & 71.1 \\
\hline 1271 & $6: 41: 09.9$ & $9: 29: 45.9$ & 0.50 & - & $06410986+0929458$ & - & 6068 & - & - & - & $<1.5$ \\
\hline 1272 & 6:41:09.9 & $9: 35: 41.1$ & 1.00 & - & - & - & - & - & - & - & $<0.8$ \\
\hline 1273 & $6: 41: 09.9$ & $9: 29: 36.8$ & 1.00 & - & - & - & 6070 & - & - & - & $<6.9$ \\
\hline $1274 *$ & 6:41:09.9 & $9: 30: 20.2$ & 0.50 & 301 & $06410992+0930202$ & - & 6071 & - & - & - & 22.0 \\
\hline 1275 & 6:41:09.9 & 9:29:34.7 & 0.50 & - & $06410992+0929347$ & - & - & - & - & - & $<6.9$ \\
\hline 1276 & $6: 41: 09.9$ & $9: 29: 43.2$ & 0.50 & - & $06410994+0929431$ & - & - & - & - & - & $<1.5$ \\
\hline $1277 *$ & $6: 41: 10.0$ & $9: 27: 46.1$ & 0.50 & 302 & $06410999+0927460$ & - & - & 115 & - & - & 311.3 \\
\hline 1278 & $6: 41: 10.0$ & $9: 40: 37.9$ & 0.50 & - & $06410999+0940379$ & - & - & - & - & - & $<1.8$ \\
\hline 1279 & $6: 41: 10.0$ & $9: 29: 35.2$ & 1.00 & - & - & - & 6073 & - & - & - & $<6.9$ \\
\hline $1280 *$ & $6: 41: 10.1$ & $9: 29: 28.6$ & 1.59 & 303 & - & - & - & - & - & - & 1.5 \\
\hline 1281 & $6: 41: 10.1$ & $9: 25: 34.1$ & 1.00 & - & - & - & 6074 & - & - & - & $<4.9$ \\
\hline 1282 & $6: 41: 10.1$ & $9: 29: 27.0$ & 0.59 & - & $06411011+0929270$ & - & - & - & - & - & $<1.7$ \\
\hline 1283 & 6:41:10.1 & $9: 33: 42.8$ & 0.50 & - & $06411011+0933427$ & 3634 & 6075 & - & - & - & $<0.8$ \\
\hline $1284 *$ & $6: 41: 10.1$ & $9: 31: 28.5$ & 0.50 & 304 & $06411013+0931285$ & 3635 & 6078 & - & - & 381 & 4.4 \\
\hline $1285^{*}$ & $6: 41: 10.2$ & $9: 29: 33.6$ & 0.50 & 305 & $06411015+0929336$ & - & 6076 & - & - & - & 7.4 \\
\hline 1286 & $6: 41: 10.2$ & 9:31:22.4 & 0.57 & - & $06411016+0931223$ & - & 6077 & - & - & - & $<0.9$ \\
\hline $1287 *$ & $6: 41: 10.2$ & $9: 30: 31.1$ & 1.00 & 306 & - & - & 6079 & - & - & 380 & 2.5 \\
\hline 1288 & $6: 41: 10.2$ & $9: 41: 10.5$ & 0.50 & - & $06411021+0941105$ & 3636 & 6080 & - & - & - & $<2.2$ \\
\hline 1289 & $6: 41: 10.2$ & $9: 29: 29.6$ & 0.50 & - & $06411023+0929296$ & - & - & - & - & - & $<1.7$ \\
\hline $1290 *$ & $6: 41: 10.3$ & $9: 33: 25.1$ & 0.50 & 307 & $06411027+0933250$ & 3639 & 6081 & - & - & 382 & 5.4 \\
\hline $1291 *$ & $6: 41: 10.3$ & $9: 34: 22.0$ & 0.50 & 308 & $06411029+0934219$ & - & - & - & - & - & 2.6 \\
\hline 1292 & $6: 41: 10.3$ & $9: 29: 26.6$ & 0.50 & - & $06411029+0929265$ & - & - & - & - & - & $<1.7$ \\
\hline $1293 *$ & $6: 41: 10.3$ & $9: 28: 33.5$ & 1.00 & 309 & - & - & 6083 & - & - & 383 & 3.0 \\
\hline 1294 & $6: 41: 10.4$ & 9:42:06.2 & 0.50 & - & $06411040+0942062$ & 3643 & 6086 & - & - & - & $<2.7$ \\
\hline $1295^{*}$ & $6: 41: 10.4$ & $9: 34: 18.7$ & 0.50 & 310 & $06411042+0934186$ & - & - & - & - & - & 1.7 \\
\hline 1296 & $6: 41: 10.4$ & $9: 41: 41.0$ & 0.50 & - & $06411044+0941410$ & 3644 & 6087 & - & - & - & $<2.5$ \\
\hline 1297 & $6: 41: 10.5$ & $9: 39: 45.2$ & 0.50 & - & $06411053+0939452$ & - & - & - & - & - & $<1.5$ \\
\hline 1298 & $6: 41: 10.8$ & $9: 35: 14.8$ & 0.50 & - & $06411082+0935148$ & - & 6096 & - & - & - & $<0.8$ \\
\hline 1299 & $6: 41: 10.9$ & 9:34:08.3 & 0.50 & - & $06411093+0934082$ & - & - & - & - & - & $<1.0$ \\
\hline 1300 & $6: 41: 11.0$ & $9: 40: 44.1$ & 0.50 & - & $06411096+0940440$ & - & - & - & - & - & $<1.8$ \\
\hline 1301 & $6: 41: 11.0$ & $9: 25: 59.4$ & 0.57 & - & $06411096+0925593$ & - & 6101 & - & - & - & $<3.0$ \\
\hline $1302 *$ & $6: 41: 11.0$ & $9: 35: 55.7$ & 0.50 & 311 & $06411099+0935556$ & 3660 & 6102 & - & - & 387 & 19.7 \\
\hline 1303 & $6: 41: 11.0$ & $9: 36: 32.4$ & 0.50 & - & $06411101+0936323$ & - & - & - & - & - & $<0.9$ \\
\hline 1304 & $6: 41: 11.0$ & $9: 37: 43.8$ & 0.50 & - & $06411102+0937438$ & - & 6104 & - & - & - & $<1.1$ \\
\hline $1305^{*}$ & $6: 41: 11.0$ & $9: 34: 24.6$ & 1.00 & 313 & - & - & - & - & - & - & 0.9 \\
\hline 1306 & $6: 41: 11.1$ & $9: 34: 12.3$ & 0.50 & - & $06411106+0934123$ & - & 6105 & - & - & - & $<0.8$ \\
\hline $1307 *$ & $6: 41: 11.1$ & 9:42:08.4 & 0.50 & 312 & $06411106+0942083$ & 3662 & 6107 & - & - & - & 3.4 \\
\hline 1308 & $6: 41: 11.2$ & $9: 30: 23.5$ & 0.50 & - & $06411121+0930235$ & 3667 & 6112 & - & - & - & $<1.2$ \\
\hline 1309 & $6: 41: 11.3$ & $9: 29: 40.8$ & 0.51 & - & $06411125+0929408$ & - & - & - & - & - & $<1.6$ \\
\hline 1310 & $6: 41: 11.3$ & $9: 27: 00.1$ & 1.00 & - & - & - & 6114 & - & - & - & $<2.6$ \\
\hline $1311 *$ & $6: 41: 11.3$ & $9: 30: 50.8$ & 0.50 & - & $06411132+0930508$ & - & 6115 & - & - & - & $<1.1$ \\
\hline $1312 *$ & 6:41:11.4 & $9: 29: 24.9$ & 1.43 & 314 & - & - & - & - & - & - & 1.5 \\
\hline $1313 *$ & $6: 41: 11.4$ & $9: 26: 38.1$ & 0.50 & - & $06411139+0926380$ & 3672 & 6117 & - & - & 388 & $<8.2$ \\
\hline $1314 *$ & $6: 41: 11.4$ & $9: 28: 13.8$ & 0.50 & - & $06411144+0928137$ & - & 6119 & - & - & - & $<2.0$ \\
\hline 1315 & $6: 41: 11.5$ & 9:35:07.4 & 1.00 & - & - & - & 6121 & - & - & - & $<0.8$ \\
\hline $1316^{*}$ & 6:41:11.6 & 9:29:09.8 & 2.52 & 315 & - & - & - & - & - & - & 1.8 \\
\hline $1317 *$ & $6: 41: 11.8$ & $9: 31: 12.3$ & 0.50 & 316 & $06411180+0931123$ & 3677 & 6125 & - & - & - & 2.6 \\
\hline 1318 & $6: 41: 11.8$ & $9: 35: 23.1$ & 0.50 & - & $06411184+0935230$ & - & - & - & - & - & $<0.8$ \\
\hline 1319* & $6: 41: 11.8$ & $9: 35: 31.3$ & 0.50 & 317 & $06411184+0935313$ & - & - & - & - & - & 2.5 \\
\hline $1320 *$ & $6: 41: 11.9$ & $9: 26: 31.4$ & 0.50 & 318 & $06411185+0926314$ & 3679 & 6127 & 116 & 183 & 391 & 40.0 \\
\hline
\end{tabular}


Table 3. (Continued)

\begin{tabular}{|c|c|c|c|c|c|c|c|c|c|c|c|}
\hline$\overline{\mathrm{N}}$ & $\begin{array}{c}\mathrm{RA} \\
{[\mathrm{h} \mathrm{m} \mathrm{s}]}\end{array}$ & $\begin{array}{c}\text { Dec. } \\
{[\mathrm{d} \mathrm{m} \mathrm{s}]}\end{array}$ & $\begin{array}{c}\text { Id. rad. } \\
\text { ["] }\end{array}$ & $\overline{\mathrm{ACIS}}$ & 2Mass & Reb.+ & $\overline{\text { Lamm+ }}$ & Flacc+ & 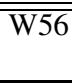 & $\overline{\overline{\text { Dahm+ }}}$ & $\begin{array}{l}\text { Ct. Rate. } \\
{\left[10^{-4} s^{-1}\right]}\end{array}$ \\
\hline 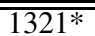 & $6: 41: 11.9$ & $9: 9: 30: 26.7$ & $\overline{0.50}$ & $\overline{-1}$ & 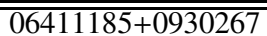 & $3 \overline{3680}$ & 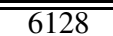 & $=$ & $\overline{-1}$ & $\overline{-1}$ & ( \\
\hline $1322 *$ & $6: 41: 11.9$ & $9: 37: 43.9$ & 0.50 & 319 & $06411190+0937438$ & 3681 & 6129 & - & - & - & 1.7 \\
\hline 1323 & $6: 41: 12.0$ & 9:29:09.0 & 0.50 & - & $06411196+0929089$ & 3683 & 6132 & - & - & - & $<1.9$ \\
\hline 1324 & $6: 41: 12.0$ & $9: 29: 55.4$ & 0.50 & - & $06411197+0929553$ & - & - & - & - & - & $<3.7$ \\
\hline 1325 & $6: 41: 12.1$ & $9: 35: 30.5$ & 0.50 & - & $06411206+0935305$ & - & - & - & - & - & $<0.8$ \\
\hline $1326 *$ & $6: 41: 12.1$ & $9: 29: 52.1$ & 0.50 & 320 & $06411212+0929521$ & 3687 & 6136 & - & - & 392 & 6.4 \\
\hline 1327 & $6: 41: 12.2$ & $9: 35: 29.5$ & 0.50 & - & $06411219+0935294$ & - & - & - & - & - & $<0.8$ \\
\hline $1328 *$ & $6: 41: 12.2$ & $9: 29: 14.8$ & 2.40 & 321 & - & - & - & - & - & - & 2.2 \\
\hline $1329 *$ & $6: 41: 12.2$ & $9: 40: 12.1$ & 0.50 & 322 & $06411224+0940121$ & 3691 & 6139 & - & - & - & 2.0 \\
\hline 1330 & $6: 41: 12.3$ & $9: 27: 57.6$ & 1.00 & - & - & - & 6141 & - & - & - & $<2.3$ \\
\hline 1331 & $6: 41: 12.4$ & $9: 39: 26.5$ & 0.50 & - & $06411236+0939264$ & 3692 & 6143 & - & 185 & - & $<1.5$ \\
\hline 1332 & $6: 41: 12.5$ & $9: 29: 09.2$ & 0.50 & - & $06411245+0929092$ & - & - & - & - & - & $<2.0$ \\
\hline $1333 *$ & $6: 41: 12.5$ & $9: 35: 08.8$ & 0.50 & 323 & $06411251+0935088$ & - & 6146 & - & - & - & 2.8 \\
\hline $1334 *$ & $6: 41: 12.6$ & 9:29:04.8 & 0.50 & 324 & $06411259+0929048$ & - & - & - & - & - & 7.8 \\
\hline 1335 & $6: 41: 12.6$ & $9: 27: 57.7$ & 1.00 & - & - & - & 6149 & - & - & - & $<2.3$ \\
\hline 1336 & $6: 41: 12.7$ & $9: 29: 10.2$ & 0.50 & - & $06411274+0929102$ & - & - & - & - & - & $<44.0$ \\
\hline $1337 *$ & $6: 41: 12.8$ & $9: 32: 28.0$ & 0.50 & - & $06411275+0932280$ & 3701 & 6154 & - & - & 395 & $<0.9$ \\
\hline 1338 & $6: 41: 12.8$ & $9: 29: 15.3$ & 0.50 & - & $06411283+0929153$ & - & - & - & - & - & $<21.2$ \\
\hline $1339 *$ & $6: 41: 12.9$ & $9: 26: 14.9$ & 0.50 & - & $06411286+0926148$ & 3706 & 6159 & $120 \dagger$ & - & 397 & $<116.2$ \\
\hline $1340 *$ & $6: 41: 12.9$ & $9: 35: 49.9$ & 0.50 & 325 & $06411293+0935499$ & - & - & - & 187 & - & 2.8 \\
\hline $1341 *$ & $6: 41: 13.0$ & $9: 35: 32.3$ & 0.50 & 326 & $06411295+0935322$ & - & 6162 & - & - & - & 9.4 \\
\hline $1342 *$ & $6: 41: 13.0$ & 9:29:08.7 & 1.37 & 327 & - & - & - & - & - & - & 50.2 \\
\hline $1343 *$ & $6: 41: 13.0$ & $9: 27: 39.3$ & 0.50 & 328 & $06411300+0927393$ & - & 6163 & $118 \dagger$ & - & 398 & 31.3 \\
\hline $1344 *$ & $6: 41: 13.0$ & $9: 27: 31.9$ & 0.50 & 329 & $06411303+0927319$ & - & 6164 & $118 \dagger$ & 189 & - & 106.3 \\
\hline $1345^{*}$ & 6:41:13.1 & $9: 31: 38.2$ & 0.50 & - & $06411306+0931382$ & 3714 & 6166 & - & - & 399 & $<1.0$ \\
\hline $1346^{*}$ & 6:41:13.1 & 9:31:06.7 & 0.50 & 330 & $06411311+0931066$ & 3717 & 6168 & - & - & 401 & 4.5 \\
\hline $1347 *$ & $6: 41: 13.1$ & $9: 28: 59.3$ & 0.50 & 331 & $06411314+0928593$ & 3718 & 6170 & - & - & 402 & 1.8 \\
\hline 1348 & $6: 41: 13.2$ & $9: 29: 20.6$ & 0.54 & - & $06411315+0929206$ & - & - & - & - & - & $<1.9$ \\
\hline $1349 *$ & $6: 41: 13.2$ & $9: 26: 10.3$ & 0.50 & 332 & $06411320+0926103$ & 3719 & 6172 & $120 \dagger$ & - & 403 & 160.3 \\
\hline 1350 & $6: 41: 13.3$ & $9: 35: 47.4$ & 0.50 & - & $06411326+0935473$ & - & 6174 & - & - & - & $<0.9$ \\
\hline $1351 *$ & $6: 41: 13.3$ & $9: 31: 50.3$ & 0.50 & 333 & $06411329+0931503$ & 3722 & 6175 & - & - & 405 & 19.1 \\
\hline 1352 & $6: 41: 13.3$ & $9: 30: 11.9$ & 0.50 & - & $06411330+0930119$ & - & 6176 & - & - & - & $<1.3$ \\
\hline $1353 *$ & $6: 41: 13.3$ & $9: 28: 07.5$ & 0.50 & 334 & $06411333+0928074$ & 3726 & 6179 & 124 & - & 407 & 19.9 \\
\hline $1354 *$ & $6: 41: 13.4$ & $9: 38: 13.8$ & 0.50 & 335 & $06411336+0938137$ & - & 6181 & - & - & - & 3.0 \\
\hline $1355^{*}$ & $6: 41: 13.4$ & $9: 30: 23.8$ & 0.50 & - & $06411340+0930237$ & 3727 & 6182 & - & - & - & $<1.3$ \\
\hline 1356 & 6:41:13.4 & $9: 33: 33.0$ & 0.50 & - & $06411340+0933329$ & - & - & - & - & - & $<0.9$ \\
\hline $1357 *$ & $6: 41: 13.4$ & $9: 27: 36.2$ & 0.50 & - & $06411341+0927362$ & - & 6183 & $118 \dagger$ & - & - & $<50.0$ \\
\hline 1358 & $6: 41: 13.4$ & $9: 40: 43.7$ & 1.00 & - & - & - & 6184 & - & - & - & $<2.0$ \\
\hline 1359 & $6: 41: 13.5$ & $9: 28: 39.3$ & 0.65 & - & $06411352+0928393$ & - & 6185 & - & - & - & $<2.0$ \\
\hline 1360 & $6: 41: 13.7$ & $9: 25: 42.8$ & 1.00 & - & - & - & 6187 & - & - & - & $<3.5$ \\
\hline $1361 *$ & $6: 41: 13.8$ & $9: 29: 32.1$ & 1.00 & - & - & 3729 & 6190 & - & - & - & $<1.6$ \\
\hline $1362 *$ & $6: 41: 13.8$ & $9: 28: 42.6$ & 1.82 & 337 & - & - & - & - & - & - & 2.8 \\
\hline $1363^{*}$ & 6:41:13.9 & $9: 40: 42.4$ & 4.74 & 336 & - & - & - & - & - & - & 2.3 \\
\hline $1364 *$ & $6: 41: 14.1$ & $9: 26: 40.6$ & 0.50 & 338 & $06411413+0926405$ & 3733 & 6201 & - & - & 408 & 39.2 \\
\hline 1365 & $6: 41: 14.2$ & $9: 38: 20.0$ & 1.00 & - & - & - & 6202 & - & - & - & $<1.4$ \\
\hline $1366^{*}$ & $6: 41: 14.2$ & 9:33:21.6 & 1.00 & - & - & - & 6203 & - & - & - & $<1.0$ \\
\hline $1367 *$ & $6: 41: 14.4$ & $9: 26: 58.3$ & 0.50 & 339 & $06411441+0926582$ & 3737 & 6207 & - & - & 410 & 4.4 \\
\hline $1368 *$ & $6: 41: 14.5$ & $9: 33: 21.4$ & 0.50 & 340 & $06411446+0933214$ & 3738 & 6209 & 126 & 191 & 411 & 67.1 \\
\hline 1369 & $6: 41: 14.5$ & $9: 32: 05.9$ & 0.50 & - & $06411447+0932058$ & - & - & - & - & - & $<1.0$ \\
\hline $1370 *$ & $6: 41: 14.5$ & $9: 37: 14.3$ & 0.50 & 341 & $06411448+0937143$ & 3739 & 6210 & 125 & - & 412 & 77.9 \\
\hline 1371 & $6: 41: 14.6$ & 9:38:06.9 & 0.50 & - & 06411464+0938069 & - & 6212 & - & - & - & $<1.4$ \\
\hline $1372 *$ & $6: 41: 14.8$ & $9: 34: 13.5$ & 0.50 & 342 & $06411475+0934134$ & 3743 & 6216 & - & - & 413 & 1.2 \\
\hline 1373 & $6: 41: 14.8$ & $9: 31: 49.9$ & 0.50 & - & $06411477+0931498$ & - & - & - & - & - & $<1.1$ \\
\hline 1374 & $6: 41: 14.8$ & $9: 37: 32.0$ & 1.00 & - & - & - & 6217 & - & - & - & $<1.3$ \\
\hline $1375^{*}$ & $6: 41: 14.8$ & $9: 32: 35.9$ & 0.50 & 343 & $06411484+0932358$ & 3746 & 6220 & 127 & - & 415 & 36.2 \\
\hline $1376^{*}$ & $6: 41: 14.9$ & $9: 25: 55.1$ & 0.50 & - & $06411485+0925550$ & - & 6219 & - & - & 414 & $<3.9$ \\
\hline 1377 & $6: 41: 14.9$ & $9: 26: 13.5$ & 0.50 & - & $06411488+0926135$ & - & 6222 & - & - & - & $<4.8$ \\
\hline 1378 & $6: 41: 15.0$ & $9: 35: 41.3$ & 0.50 & - & $06411502+0935412$ & - & - & - & - & - & $<1.0$ \\
\hline 1379 & $6: 41: 15.1$ & $9: 39: 47.2$ & 0.50 & - & $06411506+0939471$ & 3752 & 6226 & - & - & - & $<1.9$ \\
\hline $1380 *$ & $6: 41: 15.1$ & $9: 26: 44.3$ & 0.50 & 344 & $06411511+0926443$ & 3755 & 6228 & - & - & 416 & 17.4 \\
\hline
\end{tabular}


Table 3. (Continued)

\begin{tabular}{|c|c|c|c|c|c|c|c|c|c|c|c|}
\hline$\overline{\mathrm{N}}$ & $\begin{array}{c}\mathrm{RA} \\
{[\mathrm{h} \mathrm{m} \mathrm{s}]}\end{array}$ & $\begin{array}{c}\text { Dec. } \\
{[\mathrm{d} \mathrm{m} \mathrm{s}]}\end{array}$ & $\begin{array}{c}\text { Id. rad. } \\
\text { ["] } \\
\end{array}$ & $\overline{\mathrm{ACIS}}$ & 2Mass & Reb.+ & $\overline{\text { Lamm+ }}$ & Flacc+ & $\overline{\text { W56 }}$ & $\overline{\mathrm{Dahm+}}$ & $\begin{array}{l}\text { Ct. Rate. } \\
{\left[10^{-4} s^{-1}\right]}\end{array}$ \\
\hline 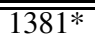 & 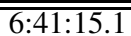 & 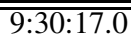 & $\overline{0.50}$ & 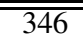 & "06411514+0930170 & (3758 & 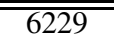 & $\overline{-1}$ & $\overline{-1}$ & $\overline{-1}$ & $\overline{\overline{1.4}}$ \\
\hline $1382 *$ & $6: 41: 15.2$ & $9: 37: 57.7$ & 0.50 & 345 & $06411521+0937576$ & - & 6232 & - & - & 417 & 10.0 \\
\hline 1383 & $6: 41: 15.3$ & $9: 31: 22.2$ & 0.50 & - & $06411528+0931222$ & - & - & - & - & - & $<1.1$ \\
\hline $1384 *$ & $6: 41: 15.3$ & $9: 31: 16.4$ & 0.50 & 347 & $06411530+0931163$ & - & - & - & 193 & - & 6.3 \\
\hline $1385^{*}$ & $6: 41: 15.4$ & $9: 25: 16.8$ & 0.50 & - & $06411537+0925167$ & 3761 & 6236 & - & - & 419 & $<11.8$ \\
\hline 1386 & $6: 41: 15.5$ & $9: 40: 12.6$ & 0.50 & - & $06411547+0940125$ & 3763 & 6240 & - & - & - & $<2.0$ \\
\hline $1387 *$ & $6: 41: 15.5$ & $9: 26: 33.5$ & 1.00 & - & - & - & 6242 & - & - & - & $<25.3$ \\
\hline $1388 *$ & $6: 41: 15.6$ & $9: 26: 33.4$ & 0.50 & 348 & $06411563+0926334$ & 3765 & 6245 & - & - & 420 & 38.9 \\
\hline 1389 & $6: 41: 15.7$ & $9: 27: 52.7$ & 0.50 & - & $06411570+0927526$ & - & - & - & - & - & $<2.4$ \\
\hline $1390 *$ & $6: 41: 15.7$ & $9: 38: 18.3$ & 0.50 & 349 & $06411571+0938182$ & 3768 & 6247 & - & 194 & - & 5.8 \\
\hline $1391 *$ & $6: 41: 15.7$ & $9: 26: 16.8$ & 0.50 & 350 & $06411574+0926168$ & 3769 & 6248 & $129 \dagger$ & - & 421 & 294.0 \\
\hline $1392 *$ & $6: 41: 15.9$ & $9: 30: 37.4$ & 0.50 & 351 & $06411587+0930373$ & - & 6249 & - & - & - & 1.6 \\
\hline $1393 *$ & $6: 41: 16.0$ & $9: 26: 09.5$ & 0.50 & - & $06411599+0926094$ & 3775 & 6251 & $129 \dagger$ & - & 422 & $<199.1$ \\
\hline $1394 *$ & $6: 41: 16.1$ & $9: 26: 43.6$ & 0.50 & - & $06411610+0926435$ & - & 6255 & - & - & - & $<3.9$ \\
\hline 1395 & $6: 41: 16.1$ & $9: 38: 16.7$ & 0.50 & - & $06411613+0938166$ & - & 6257 & - & - & - & $<1.5$ \\
\hline 1396 & $6: 41: 16.2$ & $9: 27: 10.6$ & 0.50 & - & $06411618+0927105$ & - & - & - & - & - & $<3.1$ \\
\hline 1397 & $6: 41: 16.3$ & $9: 26: 32.0$ & 8.10 & - & - & - & - & 130 & - & - & $<11.6$ \\
\hline 1398 & $6: 41: 16.4$ & $9: 39: 54.2$ & 0.50 & - & $06411642+0939542$ & - & - & - & - & - & $<1.9$ \\
\hline 1399 & $6: 41: 16.5$ & $9: 35: 22.4$ & 1.00 & - & - & - & 6264 & - & - & - & $<1.1$ \\
\hline $1400 *$ & $6: 41: 16.5$ & $9: 26: 08.3$ & 0.50 & - & $06411650+0926082$ & - & 6263 & - & - & 423 & $<4.1$ \\
\hline 1401 & $6: 41: 16.5$ & $9: 33: 49.8$ & 0.50 & - & $06411653+0933498$ & - & 6266 & - & - & - & $<1.8$ \\
\hline $1402 *$ & $6: 41: 16.7$ & $9: 29: 52.3$ & 0.50 & 352 & $06411668+0929522$ & 3789 & 6270 & 131 & - & 424 & 41.6 \\
\hline $1403 *$ & $6: 41: 16.8$ & $9: 27: 30.2$ & 0.50 & 353 & $06411678+0927301$ & 3794 & 6273 & - & - & 425 & 26.2 \\
\hline 1404 & $6: 41: 16.8$ & $9: 30: 22.5$ & 0.50 & - & $06411679+0930225$ & - & 6274 & - & - & - & $<1.5$ \\
\hline $1405^{*}$ & $6: 41: 16.8$ & $9: 36: 20.6$ & 0.50 & 354 & $06411684+0936206$ & 3795 & 6276 & 132 & - & - & 25.6 \\
\hline 1406 & $6: 41: 16.9$ & $9: 38: 50.7$ & 1.00 & - & - & - & 6278 & - & - & - & $<1.6$ \\
\hline 1407 & $6: 41: 16.9$ & $9: 27: 32.5$ & 1.00 & - & - & - & 6279 & - & - & - & $<21.7$ \\
\hline $1408 *$ & $6: 41: 17.1$ & $9: 32: 52.3$ & 0.50 & 355 & $06411705+0932523$ & 3802 & 6285 & - & - & 426 & 6.4 \\
\hline 1409 & $6: 41: 17.1$ & $9: 34: 33.5$ & 0.50 & - & $06411706+0934335$ & - & 6287 & - & - & - & $<1.1$ \\
\hline 1410 & $6: 41: 17.1$ & $9: 26: 23.7$ & 0.50 & - & $06411706+0926236$ & - & - & - & - & - & $<3.8$ \\
\hline 1411 & $6: 41: 17.1$ & $9: 26: 19.1$ & 0.50 & - & $06411707+0926191$ & - & - & - & - & - & $<3.9$ \\
\hline $1412 *$ & $6: 41: 17.1$ & 9:29:04.9 & 0.50 & - & $06411714+0929048$ & - & 6291 & - & - & - & $<2.1$ \\
\hline $1413 *$ & $6: 41: 17.1$ & $9: 30: 12.3$ & 0.50 & 356 & $06411714+0930123$ & 3808 & 6293 & - & - & - & 5.2 \\
\hline 1414 & $6: 41: 17.2$ & $9: 36: 23.2$ & 0.50 & - & $06411718+0936231$ & - & 6296 & - & - & - & $<1.2$ \\
\hline 1415 & $6: 41: 17.2$ & $9: 35: 43.1$ & 0.50 & - & $06411723+0935430$ & - & 6297 & - & - & - & $<1.1$ \\
\hline 1416 & $6: 41: 17.3$ & $9: 30: 58.5$ & 0.50 & - & $06411725+0930584$ & - & - & - & - & - & $<1.4$ \\
\hline 1417 & $6: 41: 17.3$ & $9: 26: 59.9$ & 0.50 & - & $06411727+0926598$ & - & - & - & - & - & $<3.4$ \\
\hline 1418 & $6: 41: 17.4$ & $9: 31: 52.0$ & 0.51 & - & $06411736+0931519$ & - & 6302 & - & - & - & $<1.4$ \\
\hline $1419 *$ & $6: 41: 17.4$ & $9: 37: 15.1$ & 0.69 & 358 & $06411737+0937151$ & - & 6307 & - & - & - & 1.1 \\
\hline 1420 & $6: 41: 17.4$ & $9: 35: 55.9$ & 0.50 & - & $06411738+0935559$ & - & 6306 & - & - & - & $<1.2$ \\
\hline 1421 & $6: 41: 17.4$ & 9:35:39.6 & 1.00 & - & - & - & 6305 & - & - & - & $<1.1$ \\
\hline $1422 *$ & $6: 41: 17.4$ & $9: 30: 28.7$ & 0.50 & 357 & $06411744+0930287$ & 3816 & 6308 & - & - & - & 1.6 \\
\hline 1423 & $6: 41: 17.5$ & $9: 35: 48.9$ & 1.00 & - & - & - & 6309 & - & - & - & $<1.1$ \\
\hline $1424 *$ & $6: 41: 17.5$ & $9: 28: 06.3$ & 0.50 & - & $06411750+0928063$ & - & 6312 & - & - & 429 & $<2.4$ \\
\hline 1425 & $6: 41: 17.6$ & $9: 29: 58.9$ & 0.50 & - & $06411760+0929588$ & - & - & - & - & - & $<1.8$ \\
\hline $1426^{*}$ & $6: 41: 17.7$ & $9: 29: 26.2$ & 0.50 & 359 & $06411771+0929261$ & 3820 & 6316 & 133 & - & 430 & 41.0 \\
\hline 1427 & $6: 41: 17.7$ & $9: 40: 39.6$ & 0.50 & - & $06411773+0940396$ & - & - & - & - & - & $<2.1$ \\
\hline 1428 & $6: 41: 17.7$ & $9: 34: 22.0$ & 0.50 & - & $06411774+0934219$ & - & 6317 & - & - & - & $<1.1$ \\
\hline 1429 & $6: 41: 17.9$ & $9: 41: 47.3$ & 1.00 & - & - & - & 6323 & - & - & - & $<2.8$ \\
\hline 1430 & $6: 41: 17.9$ & $9: 38: 20.4$ & 0.50 & - & $06411791+0938204$ & - & 6328 & - & - & - & $<11.4$ \\
\hline $1431 *$ & $6: 41: 17.9$ & 9:29:01.1 & 0.50 & 361 & $06411792+0929011$ & 3826 & 6326 & - & - & 431 & 7.5 \\
\hline $1432 *$ & $6: 41: 17.9$ & $9: 33: 37.0$ & 0.50 & 360 & $06411792+0933370$ & 3827 & 6327 & $134 \dagger$ & 198 & 432 & 62.7 \\
\hline 1433 & $6: 41: 18.0$ & $9: 39: 16.7$ & 0.71 & - & $06411797+0939167$ & - & 6331 & - & - & - & $<1.8$ \\
\hline 1434 & $6: 41: 18.1$ & $9: 36: 41.0$ & 0.50 & - & $06411806+0936409$ & - & 6333 & - & - & - & $<1.3$ \\
\hline $1435^{*}$ & $6: 41: 18.1$ & $9: 38: 25.4$ & 0.50 & 362 & $06411808+0938253$ & 3831 & 6334 & - & - & 433 & 13.1 \\
\hline 1436 & $6: 41: 18.1$ & 9:40:05.6 & 0.50 & - & $06411809+0940056$ & - & - & - & - & - & $<2.1$ \\
\hline 1437 & $6: 41: 18.2$ & 9:39:02.0 & 0.53 & - & $06411817+0939019$ & - & - & - & - & - & $<1.7$ \\
\hline $1438 *$ & $6: 41: 18.2$ & $9: 28: 03.1$ & 0.50 & 363 & $06411820+0928031$ & - & - & - & - & - & 4.0 \\
\hline 1439 & $6: 41: 18.2$ & $9: 33: 44.9$ & 0.50 & - & $06411822+0933448$ & - & 6337 & $134 \dagger$ & - & 434 & $<2.0$ \\
\hline $1440 *$ & $6: 41: 18.3$ & $9: 33: 53.6$ & 0.50 & 364 & $06411827+0933535$ & 3836 & 6339 & 135 & 199 & 435 & 29.3 \\
\hline
\end{tabular}


Table 3. (Continued)

\begin{tabular}{|c|c|c|c|c|c|c|c|c|c|c|c|}
\hline$\overline{\mathrm{N}}$ & $\begin{array}{c}\mathrm{RA} \\
{[\mathrm{h} \mathrm{m} \mathrm{s}]}\end{array}$ & $\begin{array}{c}\text { Dec. } \\
{[\mathrm{d} \mathrm{m} \mathrm{s}]}\end{array}$ & $\begin{array}{c}\text { Id. rad. } \\
\text { ["] }\end{array}$ & $\overline{\mathrm{ACIS}}$ & 2Mass & $\overline{\text { Reb.+ }}$ & $\overline{\text { Lamm+ }}$ & Flacc+ & $\overline{\text { W56 }}$ & $\overline{\mathrm{Dahm+}}$ & $\begin{array}{l}\text { Ct. Rate. } \\
{\left[10^{-4} s^{-1}\right]}\end{array}$ \\
\hline 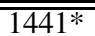 & $6: 41: 18.3$ & $9: 28: 33.0$ & 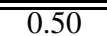 & 365 & "06411829+0928330 & 38377 & $\overline{\overline{6340}}$ & $\overline{-1}$ & $\overline{-1}$ & $\overline{4336}$ & $\overline{13.0}$ \\
\hline $1442 *$ & $6: 41: 18.3$ & $9: 29: 32.4$ & 0.50 & 366 & $06411830+0929323$ & - & - & - & - & - & 8.3 \\
\hline $1443 *$ & $6: 41: 18.4$ & 9:39:41.1 & 0.50 & 367 & $06411837+0939411$ & 3839 & 6345 & $137 \dagger$ & - & 437 & 12.6 \\
\hline $1444 *$ & 6:41:18.4 & $9: 31: 29.6$ & 0.50 & 368 & $06411839+0931296$ & 3840 & 6344 & 136 & - & - & 16.9 \\
\hline 1445 & $6: 41: 18.5$ & $9: 27: 11.6$ & 0.50 & - & $06411849+0927116$ & - & - & - & - & - & $<6.2$ \\
\hline 1446 & $6: 41: 18.7$ & 9:32:51.9 & 0.78 & - & $06411870+0932519$ & - & 6355 & - & - & - & $<1.2$ \\
\hline 1447 & $6: 41: 18.7$ & $9: 34: 25.6$ & 1.00 & - & - & - & 6356 & - & - & - & $<1.2$ \\
\hline 1448 & $6: 41: 18.8$ & $9: 36: 48.2$ & 1.00 & - & - & - & 6357 & - & - & - & $<1.4$ \\
\hline 1449 & $6: 41: 18.8$ & $9: 35: 19.1$ & 1.00 & - & - & - & 6359 & - & - & - & $<1.1$ \\
\hline $1450 *$ & $6: 41: 18.8$ & 9:30:19.9 & 0.50 & 369 & $06411882+0930199$ & 3847 & 6362 & - & - & - & 1.6 \\
\hline 1451 & $6: 41: 18.9$ & $9: 39: 44.0$ & 0.50 & - & $06411889+0939440$ & 3848 & 6365 & $137 \dagger$ & - & - & $<2.0$ \\
\hline $1452 *$ & $6: 41: 18.9$ & $9: 35: 54.2$ & 0.50 & 371 & $06411890+0935541$ & 3849 & 6364 & - & - & - & 1.6 \\
\hline $1453 *$ & $6: 41: 18.9$ & $9: 27: 15.8$ & 0.50 & 370 & $06411893+0927157$ & 3850 & 6366 & - & - & 438 & 14.5 \\
\hline 1454 & $6: 41: 19.0$ & $9: 32: 56.1$ & 0.83 & - & $06411896+0932560$ & - & 6367 & - & - & - & $<1.2$ \\
\hline $1455^{*}$ & 6:41:19.1 & $9: 26: 29.4$ & 0.50 & - & $06411911+0926294$ & 3853 & 6376 & - & - & 439 & $<3.0$ \\
\hline 1456 & $6: 41: 19.1$ & $9: 33: 37.0$ & 0.50 & - & $06411911+0933369$ & - & 6377 & - & - & - & $<2.7$ \\
\hline 1457 & $6: 41: 19.1$ & $9: 33: 26.3$ & 1.00 & - & - & - & 6378 & - & - & - & $<2.3$ \\
\hline 1458 & $6: 41: 19.1$ & $9: 35: 53.5$ & 1.00 & - & - & - & 6379 & - & - & - & $<1.6$ \\
\hline 1459 & $6: 41: 19.2$ & $9: 38: 18.6$ & 1.00 & - & - & - & 6380 & - & - & - & $<1.8$ \\
\hline 1460 & $6: 41: 19.2$ & 9:33:06.6 & 0.69 & - & $06411921+0933066$ & - & 6384 & - & - & - & $<1.2$ \\
\hline 1461 & $6: 41: 19.2$ & 9:34:49.1 & 0.50 & - & $06411921+0934491$ & - & 6381 & - & - & - & $<1.2$ \\
\hline $1462 *$ & $6: 41: 19.3$ & $9: 30: 48.8$ & 0.50 & 372 & $06411926+0930487$ & 3856 & 6383 & - & - & 440 & 1.9 \\
\hline $1463 *$ & $6: 41: 19.3$ & 9:38:07.1 & 0.50 & 373 & $06411928+0938071$ & 3857 & 6387 & - & - & - & 1.6 \\
\hline $1464 *$ & $6: 41: 19.5$ & $9: 30: 28.6$ & 0.50 & 374 & $06411945+0930286$ & 3862 & 6390 & - & - & - & 1.8 \\
\hline 1465 & $6: 41: 19.5$ & 9:34:08.9 & 1.00 & - & - & - & 6392 & - & - & - & $<1.2$ \\
\hline 1466 & $6: 41: 19.5$ & 9:39:38.1 & 0.65 & - & $06411952+0939380$ & - & 6395 & - & - & - & $<2.0$ \\
\hline 1467 & $6: 41: 19.6$ & $9: 37: 50.8$ & 1.00 & - & - & - & 6394 & - & - & - & $<1.6$ \\
\hline 1468 & $6: 41: 19.6$ & $9: 27: 24.8$ & 0.50 & - & $06411957+0927247$ & 3864 & - & - & 203 & - & $<3.1$ \\
\hline $1469 *$ & $6: 41: 19.6$ & $9: 31: 44.4$ & 0.50 & 375 & $06411963+0931443$ & 3865 & 6399 & $138 \dagger$ & - & 441 & 127.6 \\
\hline 1470 & $6: 41: 19.7$ & $9: 31: 54.2$ & 0.52 & - & $06411967+0931542$ & - & 6401 & - & - & - & $<1.6$ \\
\hline 1471 & $6: 41: 19.7$ & $9: 36: 24.0$ & 1.00 & - & - & - & 6402 & - & - & - & $<1.3$ \\
\hline 1472 & 6:41:19.7 & $9: 26: 49.4$ & 0.50 & - & $06411969+0926493$ & - & - & - & - & - & $<3.4$ \\
\hline $1473 *$ & $6: 41: 19.8$ & $9: 31: 38.2$ & 1.98 & 376 & - & - & - & $138 \dagger$ & - & - & 1.9 \\
\hline $1474 *$ & $6: 41: 19.8$ & $9: 27: 04.8$ & 0.50 & 377 & $06411984+0927048$ & 3869 & 6407 & - & - & 442 & 3.5 \\
\hline 1475 & $6: 41: 20.0$ & $9: 28: 45.7$ & 1.00 & - & - & - & 6411 & - & - & - & $<2.3$ \\
\hline $1476^{*}$ & $6: 41: 20.0$ & 9:32:07.4 & 2.75 & 378 & - & - & - & - & - & - & 1.2 \\
\hline 1477 & $6: 41: 20.1$ & $9: 36: 12.0$ & 1.00 & - & - & - & 6414 & - & - & - & $<1.4$ \\
\hline 1478 & $6: 41: 20.1$ & $9: 28: 34.8$ & 0.50 & - & $06412006+0928347$ & - & - & - & - & - & $<2.4$ \\
\hline 1479 & 6:41:20.1 & 9:34:07.3 & 1.00 & - & - & - & 6418 & - & - & - & $<1.3$ \\
\hline 1480 & $6: 41: 20.1$ & $9: 35: 36.1$ & 0.50 & - & $06412012+0935360$ & 3872 & 6420 & - & - & - & $<1.4$ \\
\hline 1481 & 6:41:20.4 & $9: 39: 52.9$ & 1.00 & - & - & - & 6431 & - & - & - & $<2.2$ \\
\hline 1482 & $6: 41: 20.5$ & 9:36:29.7 & 0.60 & - & $06412052+0936297$ & - & 6437 & - & - & - & $<1.4$ \\
\hline $1483^{*}$ & 6:41:20.6 & $9: 38: 10.3$ & 3.55 & 379 & - & - & - & - & - & - & 1.5 \\
\hline 1484 & $6: 41: 20.7$ & $9: 35: 17.0$ & 0.50 & - & $06412067+0935169$ & - & 6450 & - & - & - & $<1.3$ \\
\hline $1485^{*}$ & $6: 41: 20.7$ & $9: 28: 45.3$ & 0.50 & - & $06412070+0928452$ & 3886 & 6451 & - & - & 444 & $<2.3$ \\
\hline 1486 & $6: 41: 20.7$ & $9: 31: 40.7$ & 0.50 & - & $06412072+0931407$ & - & 6452 & - & - & - & $<1.7$ \\
\hline $1487 *$ & $6: 41: 20.7$ & 9:32:00.7 & 2.04 & 380 & - & - & - & - & - & - & 3.0 \\
\hline $1488 *$ & $6: 41: 20.7$ & 9:30:12.0 & 0.50 & 381 & $06412074+0930119$ & 3887 & 6455 & - & - & - & 23.3 \\
\hline 1489 & $6: 41: 20.8$ & $9: 26: 47.7$ & 1.00 & - & - & - & 6458 & - & - & - & $<3.2$ \\
\hline $1490 *$ & 6:41:20.9 & $9: 34: 44.8$ & 0.50 & - & $06412085+0934448$ & 3888 & 6461 & - & - & 446 & $<1.3$ \\
\hline 1491 & $6: 41: 20.9$ & $9: 34: 39.4$ & 0.50 & - & $06412090+0934394$ & - & 6463 & - & - & - & $<1.3$ \\
\hline $1492 *$ & $6: 41: 20.9$ & $9: 34: 18.1$ & 0.59 & - & $06412093+0934181$ & 3891 & 6464 & - & - & 445 & $<1.3$ \\
\hline $1493 *$ & $6: 41: 21.0$ & $9: 33: 36.2$ & 0.50 & 383 & $06412100+0933361$ & 3893 & 6467 & 141 & 204 & 447 & 54.7 \\
\hline 1494 & $6: 41: 21.0$ & 9:36:59.7 & 0.50 & - & $06412102+0936596$ & 3894 & 6469 & - & - & - & $<1.5$ \\
\hline $1495^{*}$ & $6: 41: 21.0$ & $9: 32: 41.7$ & 0.50 & 382 & $06412104+0932417$ & 3895 & 6468 & - & - & - & 1.7 \\
\hline $1496^{*}$ & $6: 41: 21.2$ & $9: 32: 14.6$ & 0.50 & 384 & $06412119+0932146$ & - & 6476 & 142 & - & 448 & 15.5 \\
\hline 1497 & $6: 41: 21.2$ & $9: 28: 31.3$ & 0.50 & - & $06412124+0928312$ & - & - & - & - & - & $<2.4$ \\
\hline 1498 & $6: 41: 21.3$ & 9:39:39.6 & 1.00 & - & - & - & 6479 & - & - & - & $<2.2$ \\
\hline 1499 & $6: 41: 21.3$ & $9: 33: 32.8$ & 1.00 & - & - & - & 6481 & - & - & - & $<2.6$ \\
\hline 1500 & $6: 41: 21.3$ & $9: 35: 24.5$ & 0.50 & - & $06412131+0935244$ & - & 6485 & - & - & - & $<1.3$ \\
\hline
\end{tabular}


Table 3. (Continued)

\begin{tabular}{|c|c|c|c|c|c|c|c|c|c|c|c|}
\hline$\overline{\mathrm{N}}$ & $\begin{array}{c}\mathrm{RA} \\
{[\mathrm{h} \mathrm{m} \mathrm{s}]}\end{array}$ & $\begin{array}{c}\text { Dec. } \\
{[\mathrm{d} \mathrm{m} \mathrm{s}]}\end{array}$ & $\begin{array}{c}\text { Id. rad. } \\
\text { ["] }\end{array}$ & $\overline{\mathrm{ACIS}}$ & 2Mass & Reb.+ & $\overline{\text { Lamm+ }}$ & Flacc+ & $\overline{\text { W56 }}$ & $\overline{\overline{\text { Dahm+ }}}$ & $\begin{array}{l}\text { Ct. Rate. } \\
{\left[10^{-4} s^{-1}\right]}\end{array}$ \\
\hline$\overline{1501}$ & $6: 41: 21.5$ & 9:35:52.2 & $\overline{1.00}$ & - & - & $\overline{-}$ & 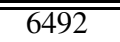 & - & - & $\overline{-}$ & $\overline{<<1.4}$ \\
\hline 1502 & $6: 41: 21.8$ & $9: 34: 59.6$ & 1.00 & - & - & - & 6505 & - & - & - & $<1.4$ \\
\hline $1503 *$ & $6: 41: 21.8$ & $9: 30: 40.0$ & 2.90 & 385 & - & - & - & - & - & - & 1.5 \\
\hline 1504 & $6: 41: 22.0$ & $9: 40: 00.5$ & 0.65 & - & $06412196+0940004$ & - & 6512 & - & - & - & $<2.4$ \\
\hline 1505 & $6: 41: 22.0$ & 9:35:09.0 & 0.50 & - & $06412199+0935089$ & - & 6516 & - & - & - & $<1.4$ \\
\hline 1506 & $6: 41: 22.0$ & 9:32:23.7 & 1.00 & - & - & - & 6514 & - & - & - & $<1.5$ \\
\hline 1507 & $6: 41: 22.0$ & 9:34:29.0 & 1.00 & - & - & - & 6515 & - & - & - & $<1.4$ \\
\hline 1508 & $6: 41: 22.0$ & $9: 33: 49.1$ & 0.58 & - & $06412202+0933491$ & - & 6518 & - & - & - & $<1.5$ \\
\hline 1509 & $6: 41: 22.2$ & $9: 35: 24.2$ & 0.50 & - & $06412215+0935241$ & - & 6526 & - & - & - & $<1.4$ \\
\hline 1510 & $6: 41: 22.2$ & $9: 30: 52.8$ & 1.00 & - & - & - & 6529 & - & - & - & $<1.9$ \\
\hline 1511 & $6: 41: 22.2$ & $9: 37: 30.4$ & 1.00 & - & - & - & 6532 & - & - & - & $<1.8$ \\
\hline 1512 & $6: 41: 22.4$ & $9: 31: 21.5$ & 1.00 & - & - & - & 6539 & - & - & - & $<1.7$ \\
\hline 1513 & $6: 41: 22.5$ & $9: 34: 33.7$ & 1.00 & - & - & - & 6542 & - & - & - & $<1.5$ \\
\hline 1514 & $6: 41: 22.5$ & 9:38:01.3 & 1.00 & - & - & - & 6544 & - & - & - & $<2.0$ \\
\hline 1515 & $6: 41: 22.5$ & $9: 35: 36.2$ & 1.00 & - & - & - & 6546 & - & - & - & $<1.4$ \\
\hline 1516 & $6: 41: 22.5$ & 9:38:12.7 & 1.00 & - & - & - & 6547 & - & - & - & $<2.1$ \\
\hline 1517 & $6: 41: 22.5$ & 9:29:12.9 & 0.50 & - & $06412253+0929129$ & 3923 & 6549 & - & - & - & $<2.2$ \\
\hline 1518 & $6: 41: 22.6$ & $9: 25: 24.4$ & 0.50 & - & $06412259+0925243$ & - & - & - & - & - & $<3.6$ \\
\hline 1519 & $6: 41: 22.7$ & $9: 30: 43.1$ & 1.00 & - & - & - & 6558 & - & - & - & $<2.0$ \\
\hline 1520 & $6: 41: 22.7$ & 9:39:09.7 & 1.00 & - & - & - & 6560 & - & - & - & $<2.2$ \\
\hline 1521 & $6: 41: 22.7$ & $9: 32: 32.6$ & 1.00 & - & - & - & 6563 & - & - & - & $<1.6$ \\
\hline 1522 & $6: 41: 22.7$ & 9:40:15.0 & 1.00 & - & - & - & 6564 & - & - & - & $<2.6$ \\
\hline 1523 & $6: 41: 22.7$ & $9: 40: 54.5$ & 1.00 & - & - & - & 6565 & - & - & - & $<2.9$ \\
\hline 1524 & $6: 41: 22.8$ & 9:32:14.8 & 1.00 & - & - & - & 6571 & - & - & - & $<1.7$ \\
\hline $1525^{*}$ & $6: 41: 22.8$ & 9:32:39.4 & 0.50 & - & $06412277+0932394$ & 3926 & 6572 & - & - & - & $<1.6$ \\
\hline $1526^{*}$ & $6: 41: 22.8$ & 9:29:38.9 & 0.50 & 386 & $06412280+0929389$ & 3927 & 6573 & - & - & - & 19.3 \\
\hline 1527 & $6: 41: 22.8$ & $9: 36: 10.8$ & 0.50 & - & $06412282+0936108$ & 3928 & 6574 & - & - & - & $<1.5$ \\
\hline $1528 *$ & $6: 41: 23.0$ & $9: 29: 30.8$ & 0.50 & 387 & $06412297+0929307$ & - & 6577 & - & - & - & 2.9 \\
\hline $1529 *$ & $6: 41: 23.0$ & $9: 27: 26.7$ & 0.50 & 388 & $06412303+0927266$ & 3935 & 6580 & 146 & 208 & - & 162.3 \\
\hline 1530 & $6: 41: 23.1$ & 9:33:03.9 & 0.50 & - & $06412305+0933039$ & - & 6583 & - & - & - & $<1.7$ \\
\hline 1531 & $6: 41: 23.1$ & $9: 31: 25.3$ & 0.50 & - & $06412313+0931253$ & 3941 & 6588 & - & - & - & $<1.9$ \\
\hline $1532 *$ & $6: 41: 23.1$ & $9: 37: 33.9$ & 0.50 & 389 & $06412313+0937338$ & 3940 & 6589 & - & - & 452 & 13.4 \\
\hline $1533^{*}$ & $6: 41: 23.2$ & $9: 35: 22.7$ & 0.50 & 390 & $06412316+0935226$ & 3942 & 6592 & - & - & - & 1.9 \\
\hline $1534 *$ & $6: 41: 23.2$ & $9: 30: 36.4$ & 0.50 & 391 & $06412320+0930364$ & 3944 & 6595 & - & - & 453 & 37.6 \\
\hline 1535 & $6: 41: 23.2$ & 9:39:46.9 & 0.59 & - & $06412322+0939469$ & - & 6603 & - & - & - & $<2.4$ \\
\hline 1536 & $6: 41: 23.2$ & $9: 38: 46.5$ & 0.50 & - & $06412323+0938464$ & 3945 & 6602 & - & - & - & $<2.1$ \\
\hline 1537 & $6: 41: 23.2$ & $9: 40: 14.8$ & 1.00 & - & - & - & 6600 & - & - & - & $<2.8$ \\
\hline 1538 & $6: 41: 23.2$ & 9:38:39.8 & 1.00 & - & - & - & 6601 & - & - & - & $<2.0$ \\
\hline 1539 & $6: 41: 23.3$ & $9: 33: 31.7$ & 1.00 & - & - & - & 6604 & - & - & - & $<2.2$ \\
\hline 1540 & $6: 41: 23.3$ & $9: 32: 30.3$ & 1.00 & - & - & - & 6607 & - & - & - & $<1.7$ \\
\hline 1541 & $6: 41: 23.3$ & 9:40:01.0 & 0.50 & - & $06412333+0940010$ & - & 6611 & - & - & - & $<2.6$ \\
\hline $1542 *$ & $6: 41: 23.3$ & 9:38:04.4 & 0.50 & 392 & $06412333+0938044$ & 3948 & 6610 & - & - & - & 4.4 \\
\hline 1543 & $6: 41: 23.4$ & $9: 27: 57.0$ & 0.71 & - & $06412338+0927570$ & - & 6620 & - & - & - & $<2.7$ \\
\hline 1544 & $6: 41: 23.4$ & $9: 34: 48.6$ & 1.00 & - & - & - & 6612 & - & - & - & $<1.6$ \\
\hline 1545 & $6: 41: 23.4$ & $9: 37: 40.2$ & 1.00 & - & - & - & 6613 & - & - & - & $<5.3$ \\
\hline 1546 & $6: 41: 23.4$ & $9: 38: 42.6$ & 0.50 & - & $06412343+0938425$ & - & 6619 & - & - & - & $<2.0$ \\
\hline 1547 & 6:41:23.4 & $9: 36: 33.1$ & 1.00 & - & - & - & 6618 & - & - & - & $<1.6$ \\
\hline 1548 & $6: 41: 23.5$ & $9: 37: 25.7$ & 1.00 & - & - & - & 6621 & - & - & - & $<2.0$ \\
\hline $1549 *$ & $6: 41: 23.5$ & $9: 41: 39.1$ & 7.75 & 393 & - & - & - & - & - & - & 4.9 \\
\hline 1550 & $6: 41: 23.5$ & $9: 33: 27.1$ & 1.00 & - & - & - & 6628 & - & - & - & $<2.9$ \\
\hline 1551 & $6: 41: 23.5$ & $9: 41: 10.5$ & 1.00 & - & - & - & 6630 & - & - & - & $<3.0$ \\
\hline 1552 & $6: 41: 23.6$ & $9: 30: 12.5$ & 0.50 & - & $06412355+0930125$ & - & 6632 & - & - & - & $<2.3$ \\
\hline 1553 & $6: 41: 23.6$ & $9: 40: 09.3$ & 1.00 & - & - & - & 6640 & - & - & - & $<2.8$ \\
\hline 1554 & $6: 41: 23.6$ & 9:33:49.7 & 0.50 & - & $06412363+0933497$ & - & 6639 & - & - & 456 & $<1.6$ \\
\hline 1555 & $6: 41: 23.6$ & $9: 32: 40.0$ & 0.50 & - & $06412363+0932399$ & - & 6641 & - & - & - & $<1.7$ \\
\hline $1556^{*}$ & $6: 41: 23.8$ & $9: 33: 56.5$ & 0.50 & 394 & $06412378+0933565$ & - & 6648 & - & 209 & - & 4.0 \\
\hline 1557 & $6: 41: 23.8$ & $9: 40: 21.3$ & 0.50 & - & $06412381+0940212$ & - & 6651 & - & - & - & $<3.0$ \\
\hline 1558 & $6: 41: 23.9$ & $9: 27: 39.3$ & 0.50 & - & $06412390+0927393$ & - & 6654 & - & - & - & $<3.3$ \\
\hline 1559 & $6: 41: 23.9$ & $9: 38: 25.4$ & 1.00 & - & - & - & 6655 & - & - & - & $<2.0$ \\
\hline 1560 & $6: 41: 24.0$ & $9: 37: 50.1$ & 0.50 & - & $06412397+0937501$ & - & 6659 & - & - & - & $<2.3$ \\
\hline
\end{tabular}


Table 3. (Continued)

\begin{tabular}{|c|c|c|c|c|c|c|c|c|c|c|c|}
\hline$\overline{\mathrm{N}}$ & $\begin{array}{c}\mathrm{RA} \\
{[\mathrm{h} \mathrm{m} \mathrm{s}]}\end{array}$ & $\begin{array}{c}\text { Dec. } \\
{[\mathrm{d} \mathrm{m} \mathrm{s}]}\end{array}$ & $\begin{array}{c}\text { Id. rad. } \\
\text { ["] }\end{array}$ & ACIS & 2Mass & $\overline{\text { Reb.+ }}$ & $\overline{\text { Lamm+ }}$ & Flacc+ & $\overline{\text { W56 }}$ & $\overline{\mathrm{Dahm+}}$ & $\begin{array}{l}\text { Ct. Rate. } \\
{\left[10^{-4} s^{-1}\right]}\end{array}$ \\
\hline 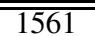 & 6 6:41:24.0 & $9: 36: 44.8$ & $\overline{0.50}$ & $\overline{-1}$ & "06412397+0936447 & 39662 & $\overline{\overline{66661}}$ & $\overline{-1}$ & $\overline{-1}$ & 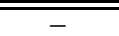 & 安1.6 \\
\hline 1562 & $6: 41: 24.0$ & 9:35:01.7 & 0.50 & - & $06412399+0935016$ & - & 6663 & - & - & - & $<1.7$ \\
\hline $1563 *$ & $6: 41: 24.0$ & $9: 26: 52.5$ & 0.50 & - & $06412402+0926525$ & - & 6668 & - & - & - & $<3.4$ \\
\hline 1564 & $6: 41: 24.2$ & $9: 38: 17.8$ & 0.60 & - & $06412415+0938178$ & - & 6677 & - & - & - & $<2.1$ \\
\hline 1565 & $6: 41: 24.2$ & $9: 37: 13.2$ & 1.00 & - & - & - & 6675 & - & - & - & $<2.1$ \\
\hline 1566 & $6: 41: 24.2$ & 9:39:50.7 & 1.00 & - & - & - & 6676 & - & - & - & $<2.5$ \\
\hline 1567 & $6: 41: 24.2$ & 9:38:35.7 & 0.50 & - & $06412423+0938357$ & - & 6686 & - & - & - & $<2.0$ \\
\hline 1568 & $6: 41: 24.2$ & $9: 32: 29.6$ & 1.00 & - & - & - & 6684 & - & - & - & $<1.7$ \\
\hline $1569 *$ & $6: 41: 24.3$ & $9: 31: 54.2$ & 0.50 & 395 & $06412425+0931541$ & 3969 & 6685 & - & - & - & 40.0 \\
\hline 1570 & $6: 41: 24.3$ & $9: 40: 30.7$ & 1.00 & - & - & - & 6687 & - & - & - & $<3.1$ \\
\hline 1571 & $6: 41: 24.3$ & 9:34:46.1 & 0.50 & - & $06412434+0934461$ & - & 6693 & - & - & - & $<1.7$ \\
\hline $1572 *$ & $6: 41: 24.5$ & 9:32:45.1 & 0.50 & 396 & $06412445+0932451$ & 3977 & 6699 & $148 \dagger$ & - & 459 & 26.9 \\
\hline 1573 & $6: 41: 24.5$ & 9:35:02.0 & 1.00 & - & - & - & 6703 & - & - & - & $<1.7$ \\
\hline $1574 *$ & $6: 41: 24.5$ & $9: 37: 35.5$ & 0.50 & 397 & $06412454+0937355$ & 3980 & 6706 & - & - & 460 & 14.2 \\
\hline 1575 & $6: 41: 24.6$ & 9:34:39.9 & 1.00 & - & - & - & 6707 & - & - & - & $<1.7$ \\
\hline 1576 & $6: 41: 24.6$ & $9: 32: 49.3$ & 0.50 & - & $06412459+0932493$ & - & 6709 & $148 \dagger$ & - & - & $<23.0$ \\
\hline 1577 & $6: 41: 24.6$ & 9:38:28.6 & 1.00 & - & - & - & 6710 & - & - & - & $<2.1$ \\
\hline 1578 & $6: 41: 24.7$ & 9:32:39.0 & 1.00 & - & - & - & 6713 & - & - & - & $<1.7$ \\
\hline $1579 *$ & $6: 41: 24.7$ & $9: 26: 23.4$ & 0.50 & 398 & $06412473+0926233$ & 3982 & 6716 & - & 210 & - & 3.1 \\
\hline 1580 & $6: 41: 24.8$ & $9: 39: 50.2$ & 0.50 & - & $06412475+0939502$ & - & 6720 & - & - & - & $<2.5$ \\
\hline 1581 & $6: 41: 24.8$ & $9: 41: 40.0$ & 1.00 & - & - & - & 6718 & - & - & - & $<14.3$ \\
\hline 1582 & $6: 41: 24.8$ & 9:39:36.4 & 1.00 & - & - & - & 6719 & - & - & - & $<2.5$ \\
\hline 1583 & $6: 41: 24.8$ & $9: 31: 19.1$ & 1.00 & - & - & - & 6721 & - & - & - & $<2.1$ \\
\hline 1584 & $6: 41: 24.8$ & $9: 32: 21.2$ & 0.50 & - & $06412480+0932211$ & - & 6725 & - & - & - & $<1.8$ \\
\hline 1585 & $6: 41: 24.9$ & $9: 38: 39.0$ & 1.00 & - & - & - & 6730 & - & - & - & $<2.2$ \\
\hline 1586 & $6: 41: 24.9$ & 9:36:16.6 & 1.00 & - & - & - & 6732 & - & - & - & $<1.8$ \\
\hline 1587 & $6: 41: 25.0$ & $9: 39: 47.5$ & 0.50 & - & $06412498+0939475$ & 3984 & 6735 & - & - & - & $<2.5$ \\
\hline 1588 & $6: 41: 25.0$ & $9: 31: 58.4$ & 1.00 & - & - & - & 6737 & - & - & - & $<2.0$ \\
\hline 1589 & $6: 41: 25.1$ & $9: 30: 54.0$ & 1.00 & - & - & - & 6740 & - & - & - & $<2.2$ \\
\hline 1590 & $6: 41: 25.1$ & 9:35:07.4 & 1.00 & - & - & - & 6741 & - & - & - & $<1.8$ \\
\hline $1591 *$ & $6: 41: 25.1$ & $9: 36: 00.2$ & 0.50 & 399 & $06412508+0936001$ & 3987 & 6743 & - & - & - & 4.5 \\
\hline 1592 & $6: 41: 25.1$ & $9: 38: 42.6$ & 1.00 & - & - & - & 6745 & - & - & - & $<2.3$ \\
\hline 1593 & $6: 41: 25.2$ & $9: 39: 35.9$ & 1.00 & - & - & - & 6749 & - & - & - & $<2.5$ \\
\hline 1594 & $6: 41: 25.2$ & $9: 40: 28.8$ & 1.00 & - & - & - & 6750 & - & - & - & $<3.1$ \\
\hline 1595 & $6: 41: 25.2$ & 9:32:39.4 & 1.00 & - & - & - & 6755 & - & - & - & $<1.8$ \\
\hline 1596 & $6: 41: 25.3$ & $9: 38: 23.9$ & 1.00 & - & - & - & 6759 & - & - & - & $<2.2$ \\
\hline 1597 & $6: 41: 25.4$ & $9: 32: 25.3$ & 1.00 & - & - & - & 6762 & - & - & - & $<2.0$ \\
\hline 1598 & $6: 41: 25.4$ & $9: 36: 54.3$ & 1.00 & - & - & - & 6763 & - & - & - & $<1.8$ \\
\hline 1599 & $6: 41: 25.4$ & $9: 30: 22.9$ & 1.00 & - & - & - & 6766 & - & - & - & $<2.3$ \\
\hline 1600 & $6: 41: 25.4$ & $9: 32: 50.4$ & 0.50 & - & $06412543+0932503$ & - & 6770 & - & - & - & $<1.9$ \\
\hline 1601 & $6: 41: 25.4$ & $9: 39: 27.7$ & 1.00 & - & - & - & 6773 & - & - & - & $<2.5$ \\
\hline 1602 & $6: 41: 25.5$ & $9: 31: 00.9$ & 0.50 & - & $06412548+0931008$ & 3994 & 6776 & - & - & - & $<2.1$ \\
\hline 1603 & $6: 41: 25.5$ & $9: 26: 20.1$ & 0.50 & - & $06412549+0926200$ & - & 6775 & - & - & - & $<3.6$ \\
\hline 1604 & $6: 41: 25.5$ & $9: 33: 12.7$ & 1.00 & - & - & - & 6777 & - & - & - & $<3.5$ \\
\hline 1605 & $6: 41: 25.5$ & $9: 34: 54.9$ & 1.00 & - & - & - & 6778 & - & - & - & $<1.9$ \\
\hline $1606 *$ & $6: 41: 25.6$ & $9: 34: 43.0$ & 0.50 & 400 & $06412562+0934429$ & 3995 & 6784 & $149 \dagger$ & - & 461 & 10.3 \\
\hline 1607 & $6: 41: 25.6$ & $9: 37: 46.7$ & 1.00 & - & - & - & 6782 & - & - & - & $<2.3$ \\
\hline 1608 & $6: 41: 25.6$ & $9: 31: 23.1$ & 1.00 & - & - & - & 6785 & - & - & - & $<2.2$ \\
\hline 1609 & $6: 41: 25.6$ & $9: 40: 16.6$ & 1.00 & - & - & - & 6786 & - & - & - & $<2.9$ \\
\hline 1610 & $6: 41: 25.7$ & $9: 31: 56.8$ & 1.00 & - & - & - & 6788 & - & - & - & $<2.2$ \\
\hline 1611 & $6: 41: 25.7$ & $9: 36: 40.0$ & 0.50 & - & $06412569+0936400$ & 3999 & 6792 & - & 211 & - & $<1.9$ \\
\hline $1612 *$ & $6: 41: 25.8$ & $9: 40: 36.5$ & 3.09 & 401 & - & - & - & - & - & - & 4.1 \\
\hline 1613 & $6: 41: 25.8$ & 9:33:16.7 & 1.00 & - & - & - & 6795 & - & - & - & $<3.5$ \\
\hline 1614 & $6: 41: 25.8$ & 9:41:16.7 & 1.00 & - & - & - & 6797 & - & - & - & $<3.2$ \\
\hline 1615 & $6: 41: 25.9$ & $9: 41: 00.8$ & 1.00 & - & - & - & 6799 & - & - & - & $<3.2$ \\
\hline 1616 & $6: 41: 25.9$ & $9: 36: 07.5$ & 1.00 & - & - & - & 6803 & - & - & - & $<1.9$ \\
\hline 1617 & $6: 41: 25.9$ & $9: 36: 47.9$ & 1.00 & - & - & - & 6804 & - & - & - & $<1.9$ \\
\hline $1618 *$ & $6: 41: 25.9$ & $9: 30: 26.1$ & 0.50 & - & $06412593+0930260$ & 4001 & 6805 & - & - & - & $<2.3$ \\
\hline 1619 & $6: 41: 26.0$ & $9: 33: 46.3$ & 1.00 & - & - & - & 6812 & - & - & - & $<1.8$ \\
\hline 1620 & $6: 41: 26.1$ & $9: 27: 44.4$ & 0.55 & - & $06412607+0927443$ & - & - & - & - & - & $<3.0$ \\
\hline
\end{tabular}


Table 3. (Continued)

\begin{tabular}{|c|c|c|c|c|c|c|c|c|c|c|c|}
\hline$\overline{\mathrm{N}}$ & $\begin{array}{c}\mathrm{RA} \\
{[\mathrm{h} \mathrm{m} \mathrm{s}]}\end{array}$ & $\begin{array}{c}\text { Dec. } \\
{[\mathrm{d} \mathrm{m} \mathrm{s}]}\end{array}$ & $\begin{array}{c}\text { Id. rad. } \\
\text { ["] }\end{array}$ & $\overline{\mathrm{ACIS}}$ & 2Mass & Reb.+ & $\overline{\text { Lamm+ }}$ & Flacc+ & $\overline{\text { W56 }}$ & $\overline{\overline{\text { Dahm+ }}}$ & $\begin{array}{l}\text { Ct. Rate. } \\
{\left[10^{-4} s^{-1}\right]}\end{array}$ \\
\hline$\overline{c 1621}$ & 6 6:41:26.1 & $9: 32: 44.1$ & $\overline{\overline{1.00}}$ & - & - & $\overline{-1}$ & "6814 & - & $\overline{-1}$ & $\overline{-1}$ & 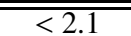 \\
\hline 1622 & $6: 41: 26.1$ & $9: 31: 48.0$ & 1.00 & - & - & - & 6815 & - & - & - & $<2.1$ \\
\hline 1623 & $6: 41: 26.1$ & $9: 29: 59.2$ & 1.00 & - & - & - & 6816 & - & - & - & $<2.5$ \\
\hline 1624 & $6: 41: 26.2$ & $9: 35: 06.9$ & 1.00 & - & - & - & 6818 & - & - & - & $<1.9$ \\
\hline 1625 & $6: 41: 26.2$ & $9: 41: 38.7$ & 1.00 & - & - & - & 6819 & - & - & - & $<15.8$ \\
\hline 1626 & $6: 41: 26.2$ & 9:37:09.1 & 0.50 & - & $06412618+0937090$ & - & 6822 & - & - & - & $<2.0$ \\
\hline 1627 & $6: 41: 26.2$ & 9:38:24.1 & 1.00 & - & - & - & 6820 & - & - & - & $<2.3$ \\
\hline 1628 & $6: 41: 26.2$ & $9: 36: 24.1$ & 1.00 & - & - & - & 6826 & - & - & - & $<2.0$ \\
\hline 1629 & $6: 41: 26.2$ & $9: 31: 34.4$ & 0.50 & - & $06412624+0931343$ & 4009 & 6827 & - & - & - & $<2.0$ \\
\hline 1630 & $6: 41: 26.3$ & $9: 34: 48.3$ & 0.50 & - & $06412628+0934482$ & 4010 & 6834 & $149 \dagger$ & - & - & $<2.0$ \\
\hline 1631 & $6: 41: 26.3$ & 9:32:05.6 & 1.00 & - & - & - & 6833 & - & - & - & $<2.1$ \\
\hline 1632 & $6: 41: 26.3$ & $9: 35: 42.6$ & 1.02 & - & $06412634+0935425$ & - & 6836 & - & - & - & $<1.8$ \\
\hline 1633 & $6: 41: 26.4$ & $9: 40: 17.2$ & 1.00 & - & - & - & 6839 & - & - & - & $<3.0$ \\
\hline 1634 & $6: 41: 26.4$ & 9:38:32.7 & 1.00 & - & - & - & 6841 & - & - & - & $<2.3$ \\
\hline 1635 & $6: 41: 26.5$ & $9: 37: 37.6$ & 0.81 & - & $06412651+0937375$ & - & 6849 & - & - & - & $<2.2$ \\
\hline 1636 & $6: 41: 26.5$ & 9:33:09.5 & 1.00 & - & - & - & 6848 & - & - & - & $<4.0$ \\
\hline 1637 & $6: 41: 26.5$ & $9: 24: 57.0$ & 0.50 & - & $06412653+0924569$ & - & - & - & - & - & $<5.8$ \\
\hline 1638 & $6: 41: 26.5$ & 9:32:14.3 & 1.00 & - & - & - & 6850 & - & - & - & $<2.1$ \\
\hline 1639 & 6:41:26.6 & $9: 39: 24.8$ & 1.00 & - & - & - & 6854 & - & - & - & $<2.7$ \\
\hline 1640 & $6: 41: 26.6$ & $9: 34: 12.5$ & 0.50 & - & $06412658+0934125$ & - & 6860 & - & - & - & $<1.9$ \\
\hline 1641 & 6:41:26.6 & $9: 35: 30.3$ & 1.00 & - & - & - & 6863 & - & - & - & $<1.9$ \\
\hline 1642 & $6: 41: 26.7$ & $9: 31: 24.8$ & 0.76 & - & $06412669+0931248$ & - & 6868 & - & - & - & $<2.1$ \\
\hline 1643 & $6: 41: 26.7$ & 9:32:04.4 & 1.00 & - & - & - & 6869 & - & - & - & $<2.6$ \\
\hline 1644 & $6: 41: 26.8$ & $9: 33: 38.4$ & 1.00 & - & - & - & 6870 & - & - & - & $<1.9$ \\
\hline 1645 & $6: 41: 26.8$ & $9: 27: 54.0$ & 0.50 & - & $06412676+0927540$ & - & 6871 & - & - & - & $<3.1$ \\
\hline 1646 & $6: 41: 26.8$ & $9: 37: 44.3$ & 1.00 & - & - & - & 6875 & - & - & - & $<2.2$ \\
\hline $1647 *$ & $6: 41: 26.8$ & $9: 27: 04.5$ & 0.50 & 404 & $06412682+0927044$ & - & 6876 & - & - & - & 6.3 \\
\hline 1648 & $6: 41: 26.9$ & $9: 35: 23.4$ & 0.50 & - & $06412688+0935234$ & 4022 & 6879 & - & - & - & $<2.0$ \\
\hline 1649 & $6: 41: 26.9$ & $9: 26: 42.2$ & 1.00 & - & - & - & 6878 & - & - & - & $<4.0$ \\
\hline 1650 & $6: 41: 26.9$ & 9:38:32.1 & 1.00 & - & - & - & 6882 & - & - & - & $<2.6$ \\
\hline $1651 *$ & $6: 41: 27.0$ & $9: 38: 40.2$ & 0.50 & 402 & $06412696+0938401$ & 4024 & 6889 & - & - & - & 2.3 \\
\hline 1652 & $6: 41: 27.0$ & 9:38:39.1 & 1.00 & - & - & - & 6885 & - & - & - & $<2.7$ \\
\hline 1653 & $6: 41: 27.0$ & $9: 30: 52.7$ & 0.50 & - & $06412699+0930526$ & 4026 & 6888 & - & - & - & $<2.2$ \\
\hline $1654 *$ & $6: 41: 27.0$ & $9: 30: 13.2$ & 0.50 & 403 & $06412700+0930131$ & 4027 & 6887 & - & - & 462 & 7.8 \\
\hline 1655 & $6: 41: 27.1$ & 9:33:05.2 & 0.50 & - & $06412710+0933052$ & 4030 & 6893 & - & - & - & $<4.3$ \\
\hline 1656 & $6: 41: 27.1$ & $9: 41: 32.7$ & 1.00 & - & - & - & 6894 & - & - & - & $<8.6$ \\
\hline $1657 *$ & $6: 41: 27.2$ & $9: 35: 06.1$ & 0.50 & 405 & $06412715+0935061$ & 4032 & 6899 & 150 & - & - & 94.4 \\
\hline $1658 *$ & $6: 41: 27.2$ & $9: 26: 29.4$ & 0.51 & - & $06412716+0926294$ & 4033 & 6902 & - & - & - & $<3.7$ \\
\hline 1659 & $6: 41: 27.2$ & 9:30:59.0 & 1.00 & - & - & - & 6903 & - & - & - & $<2.2$ \\
\hline 1660 & $6: 41: 27.2$ & $9: 31: 32.6$ & 1.00 & - & - & - & 6905 & - & - & - & $<2.2$ \\
\hline 1661 & $6: 41: 27.3$ & $9: 28: 07.3$ & 0.50 & - & $06412727+0928073$ & - & 6908 & - & - & - & $<3.1$ \\
\hline $1662 *$ & $6: 41: 27.3$ & 9:32:06.5 & 0.50 & 406 & $06412730+0932064$ & 4038 & 6913 & - & - & 463 & 3.8 \\
\hline 1663 & $6: 41: 27.3$ & $9: 26: 07.6$ & 0.50 & - & $06412733+0926076$ & - & 6916 & - & - & - & $<3.9$ \\
\hline 1664 & $6: 41: 27.4$ & $9: 32: 40.2$ & 1.00 & - & - & - & 6918 & - & - & - & $<2.2$ \\
\hline 1665 & $6: 41: 27.4$ & $9: 40: 41.5$ & 1.00 & - & - & - & 6921 & - & - & - & $<3.1$ \\
\hline 1666 & $6: 41: 27.4$ & $9: 30: 13.8$ & 1.00 & - & - & - & 6923 & - & - & - & $<5.1$ \\
\hline 1667 & $6: 41: 27.5$ & $9: 36: 51.8$ & 0.62 & - & $06412747+0936517$ & - & 6926 & - & - & - & $<2.1$ \\
\hline 1668 & $6: 41: 27.5$ & 9:39:46.1 & 1.00 & - & - & - & 6929 & - & - & - & $<3.7$ \\
\hline 1669 & $6: 41: 27.5$ & 9:31:03.6 & 1.00 & - & - & - & 6933 & - & - & - & $<2.2$ \\
\hline 1670 & $6: 41: 27.5$ & $9: 40: 14.0$ & 0.50 & - & $06412754+0940140$ & 4043 & 6935 & - & - & - & $<3.1$ \\
\hline 1671 & $6: 41: 27.5$ & 9:38:05.4 & 1.00 & - & - & - & 6934 & - & - & - & $<2.4$ \\
\hline 1672 & $6: 41: 27.6$ & $9: 31: 55.8$ & 0.50 & - & $06412756+0931558$ & 4045 & 6938 & - & - & - & $<2.2$ \\
\hline 1673 & $6: 41: 27.6$ & 9:41:06.4 & 0.82 & - & $06412756+0941063$ & - & 6940 & - & - & - & $<3.2$ \\
\hline 1674 & $6: 41: 27.6$ & 9:30:58.4 & 1.00 & - & - & - & 6937 & - & - & - & $<2.2$ \\
\hline 1675 & $6: 41: 27.6$ & $9: 25: 39.5$ & 0.50 & - & $06412758+0925395$ & - & 6941 & - & - & - & $<7.5$ \\
\hline 1676 & $6: 41: 27.7$ & $9: 31: 42.2$ & 1.00 & - & - & - & 6947 & - & - & - & $<2.3$ \\
\hline 1677 & $6: 41: 27.7$ & 9:34:22.9 & 0.50 & - & $06412772+0934228$ & 4048 & 6952 & - & - & - & $<2.1$ \\
\hline 1678 & $6: 41: 27.7$ & 9:37:00.6 & 0.89 & - & $06412772+0937006$ & 4050 & 6953 & - & - & - & $<2.2$ \\
\hline 1679 & $6: 41: 27.7$ & $9: 32: 26.4$ & 0.50 & - & $06412773+0932264$ & 4049 & 6951 & - & - & - & $<2.3$ \\
\hline 1680 & $6: 41: 27.8$ & 9:28:07.4 & 1.00 & - & - & - & 6956 & - & - & - & $<3.1$ \\
\hline
\end{tabular}


Table 3. (Continued)

\begin{tabular}{|c|c|c|c|c|c|c|c|c|c|c|c|}
\hline$\overline{\mathrm{N}}$ & $\begin{array}{c}\mathrm{RA} \\
{[\mathrm{h} \mathrm{m} \mathrm{s}]}\end{array}$ & $\begin{array}{c}\text { Dec. } \\
{[\mathrm{d} \mathrm{m} \mathrm{s}]}\end{array}$ & $\begin{array}{c}\text { Id. rad. } \\
\text { ["] }\end{array}$ & ACIS & 2Mass & $\overline{\text { Reb.+ }}$ & $\overline{\text { Lamm+ }}$ & Flacc+ & $\overline{\text { W56 }}$ & $\overline{\mathrm{Dahm+}}$ & $\begin{array}{l}\text { Ct. Rate. } \\
{\left[10^{-4} s^{-1}\right]}\end{array}$ \\
\hline 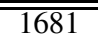 & 6 6:41:27.8 & $99: 31: 32.4$ & $\overline{0.50}$ & $\overline{-1}$ & "06412779+0931323 & $\overline{-1}$ & "6960 & - & $\overline{-1}$ & 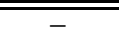 & 穴2.3 \\
\hline 1682 & $6: 41: 27.8$ & $9: 39: 41.5$ & 0.50 & - & $06412780+0939415$ & - & 6962 & - & - & - & $<3.9$ \\
\hline 1683 & $6: 41: 27.8$ & $9: 36: 07.3$ & 1.00 & - & - & - & 6963 & - & - & - & $<2.0$ \\
\hline 1684 & $6: 41: 27.9$ & $9: 31: 45.0$ & 1.00 & - & - & - & 6967 & - & - & - & $<2.3$ \\
\hline 1685 & $6: 41: 27.9$ & $9: 31: 20.8$ & 1.00 & - & - & - & 6968 & - & - & - & $<2.2$ \\
\hline 1686 & $6: 41: 27.9$ & $9: 31: 56.6$ & 0.50 & - & $06412794+0931566$ & - & 6972 & - & - & - & $<2.3$ \\
\hline 1687 & $6: 41: 27.9$ & 9:38:08.9 & 1.00 & - & - & - & 6970 & - & - & - & $<2.6$ \\
\hline 1688 & $6: 41: 28.0$ & $9: 34: 35.8$ & 0.65 & - & $06412795+0934358$ & - & 6975 & - & - & - & $<2.3$ \\
\hline 1689 & $6: 41: 28.0$ & $9: 30: 53.7$ & 1.00 & - & - & - & 6974 & - & - & - & $<2.3$ \\
\hline 1690 & 6:41:28.0 & $9: 40: 52.8$ & 0.64 & - & $06412797+0940527$ & - & 6977 & - & - & - & $<3.2$ \\
\hline 1691 & $6: 41: 28.0$ & $9: 31: 08.5$ & 1.00 & - & - & - & 6979 & - & - & - & $<2.2$ \\
\hline $1692 *$ & $6: 41: 28.1$ & 9:32:49.9 & 0.50 & 407 & $06412808+0932498$ & 4059 & 6987 & - & - & - & 11.4 \\
\hline 1693 & $6: 41: 28.1$ & 9:32:34.1 & 1.00 & - & - & - & 6986 & - & - & - & $<2.4$ \\
\hline 1694 & $6: 41: 28.2$ & $9: 28: 15.0$ & 1.00 & - & - & - & 6991 & - & - & - & $<3.1$ \\
\hline 1695 & $6: 41: 28.2$ & $9: 35: 58.8$ & 1.00 & - & - & - & 6993 & - & - & - & $<2.0$ \\
\hline 1696 & $6: 41: 28.2$ & $9: 37: 26.3$ & 1.00 & - & - & - & 6994 & - & - & - & $<2.3$ \\
\hline $1697 *$ & $6: 41: 28.2$ & $9: 32: 53.3$ & 0.50 & - & $06412819+0932533$ & - & 6996 & - & - & - & $<10.4$ \\
\hline 1698 & $6: 41: 28.2$ & $9: 37: 42.0$ & 0.73 & - & $06412823+0937420$ & - & 7000 & - & - & - & $<2.3$ \\
\hline 1699 & $6: 41: 28.3$ & $9: 34: 28.7$ & 0.50 & - & $06412826+0934286$ & 4060 & 7002 & - & - & - & $<2.2$ \\
\hline 1700 & $6: 41: 28.3$ & $9: 40: 42.2$ & 0.50 & - & $06412826+0940421$ & 4061 & 7003 & - & - & - & $<3.2$ \\
\hline 1701 & $6: 41: 28.3$ & $9: 39: 30.1$ & 0.50 & - & $06412827+0939300$ & - & 7004 & $152 \dagger$ & - & - & $<4.1$ \\
\hline 1702 & $6: 41: 28.3$ & 9:40:03.1 & 0.50 & - & $06412829+0940031$ & 4062 & 7009 & - & - & - & $<3.9$ \\
\hline 1703 & $6: 41: 28.3$ & $9: 34: 60.0$ & 0.50 & - & $06412829+0934599$ & 4063 & 7010 & - & - & - & $<2.4$ \\
\hline 1704 & $6: 41: 28.3$ & $9: 34: 56.5$ & 1.00 & - & - & - & 7008 & - & - & - & $<2.4$ \\
\hline 1705 & $6: 41: 28.3$ & $9: 32: 27.0$ & 1.00 & - & - & - & 7011 & - & - & - & $<2.4$ \\
\hline 1706 & $6: 41: 28.3$ & 9:34:29.9 & 1.00 & - & - & - & 7012 & - & - & - & $<2.1$ \\
\hline 1707 & $6: 41: 28.3$ & 9:30:54.9 & 1.00 & - & - & - & 7013 & - & - & - & $<2.3$ \\
\hline 1708 & $6: 41: 28.3$ & $9: 37: 20.2$ & 1.00 & - & - & - & 7014 & - & - & - & $<2.3$ \\
\hline 1709 & $6: 41: 28.4$ & $9: 31: 21.0$ & 1.00 & - & - & - & 7016 & - & - & - & $<2.3$ \\
\hline 1710 & $6: 41: 28.4$ & 9:33:29.6 & 1.00 & - & - & - & 7019 & - & - & - & $<2.0$ \\
\hline 1711 & $6: 41: 28.4$ & $9: 32: 42.5$ & 1.00 & - & - & - & 7021 & - & - & - & $<6.3$ \\
\hline 1712 & $6: 41: 28.5$ & $9: 33: 17.4$ & 1.00 & - & - & - & 7023 & - & - & - & $<2.4$ \\
\hline 1713 & $6: 41: 28.5$ & 9:32:13.1 & 1.00 & - & - & - & 7024 & - & - & - & $<2.3$ \\
\hline 1714 & $6: 41: 28.5$ & $9: 27: 14.7$ & 1.00 & - & - & - & 7026 & - & - & - & $<11.4$ \\
\hline 1715 & $6: 41: 28.5$ & $9: 33: 37.5$ & 1.00 & - & - & - & 7028 & - & - & - & $<2.1$ \\
\hline 1716 & $6: 41: 28.5$ & $9: 39: 17.6$ & 1.00 & - & - & - & 7029 & - & - & - & $<3.8$ \\
\hline 1717 & $6: 41: 28.5$ & $9: 37: 30.4$ & 1.00 & - & - & - & 7030 & - & - & - & $<2.3$ \\
\hline 1718 & $6: 41: 28.6$ & $9: 40: 53.7$ & 1.00 & - & - & - & 7032 & - & - & - & $<3.3$ \\
\hline 1719 & $6: 41: 28.6$ & $9: 28: 05.8$ & 1.00 & - & - & - & 7036 & - & - & - & $<3.2$ \\
\hline 1720 & $6: 41: 28.7$ & $9: 32: 34.3$ & 1.00 & - & - & - & 7042 & - & - & - & $<2.4$ \\
\hline $1721^{*}$ & $6: 41: 28.7$ & $9: 27: 10.8$ & 0.50 & 409 & $06412874+0927107$ & 4069 & 7044 & - & - & 464 & 20.5 \\
\hline 1722 & $6: 41: 28.8$ & $9: 26: 11.2$ & 0.50 & - & $06412875+0926112$ & 4070 & 7043 & - & - & - & $<23.3$ \\
\hline 1723 & $6: 41: 28.8$ & $9: 28: 15.8$ & 1.02 & - & $06412876+0928158$ & - & - & - & - & - & $<3.1$ \\
\hline 1724 & $6: 41: 28.8$ & 9:38:01.6 & 1.00 & - & - & - & 7046 & - & - & - & $<2.4$ \\
\hline $1725^{*}$ & $6: 41: 28.8$ & $9: 38: 38.8$ & 0.50 & 408 & $06412878+0938388$ & 4072 & 7049 & 153 & 213 & - & 48.5 \\
\hline 1726 & $6: 41: 28.8$ & 9:32:08.8 & 0.50 & - & $06412879+0932087$ & 4073 & 7051 & - & - & - & $<2.3$ \\
\hline $1727 *$ & $6: 41: 28.8$ & 9:34:54.1 & 0.50 & 410 & $06412884+0934540$ & 4075 & 7057 & - & - & - & 2.6 \\
\hline 1728 & $6: 41: 28.8$ & 9:34:00.6 & 1.00 & - & - & - & 7055 & - & - & - & $<2.1$ \\
\hline 1729 & $6: 41: 28.9$ & $9: 31: 44.2$ & 0.57 & - & $06412888+0931441$ & - & 7060 & - & - & - & $<2.3$ \\
\hline 1730 & 6:41:28.9 & $9: 31: 46.1$ & 1.00 & - & - & - & 7058 & - & - & - & $<2.3$ \\
\hline 1731 & $6: 41: 28.9$ & $9: 31: 49.4$ & 0.50 & - & $06412892+0931494$ & - & 7061 & - & - & - & $<2.3$ \\
\hline 1732 & $6: 41: 29.0$ & $9: 34: 21.0$ & 1.00 & - & - & - & 7063 & - & - & - & $<2.1$ \\
\hline 1733 & $6: 41: 29.0$ & $9: 29: 42.1$ & 1.00 & - & - & - & 7064 & - & - & - & $<2.6$ \\
\hline 1734 & $6: 41: 29.0$ & $9: 31: 11.8$ & 1.00 & - & - & - & 7065 & - & - & - & $<2.3$ \\
\hline 1735 & $6: 41: 29.0$ & $9: 33: 56.3$ & 0.50 & - & $06412901+0933563$ & - & 7066 & - & - & - & $<2.1$ \\
\hline 1736 & $6: 41: 29.0$ & $9: 41: 06.4$ & 1.00 & - & - & - & 7069 & - & - & - & $<3.5$ \\
\hline $1737 *$ & $6: 41: 29.1$ & 9:39:50.4 & 2.72 & 411 & - & - & - & - & - & - & 8.9 \\
\hline 1738 & $6: 41: 29.1$ & 9:34:21.9 & 1.00 & - & - & - & 7072 & - & - & - & $<2.1$ \\
\hline 1739 & $6: 41: 29.2$ & $9: 28: 48.2$ & 0.50 & - & $06412915+0928481$ & - & 7075 & - & - & - & $<2.7$ \\
\hline $1740 *$ & $6: 41: 29.2$ & $9: 39: 36.0$ & 0.50 & 412 & $06412918+0939359$ & 4081 & 7076 & $152 \dagger$ & 214 & - & 96.0 \\
\hline
\end{tabular}


Table 3. (Continued)

\begin{tabular}{|c|c|c|c|c|c|c|c|c|c|c|c|}
\hline$\overline{\mathrm{N}}$ & $\begin{array}{c}\mathrm{RA} \\
{[\mathrm{h} \mathrm{m} \mathrm{s}]}\end{array}$ & $\begin{array}{c}\text { Dec. } \\
{[\mathrm{d} \mathrm{m} \mathrm{s}]}\end{array}$ & $\begin{array}{c}\text { Id. rad. } \\
\text { ["] } \\
\end{array}$ & $\overline{\mathrm{ACIS}}$ & 2Mass & Reb.+ & $\overline{\text { Lamm+ }}$ & Flacc+ & $\overline{\text { W56 }}$ & $\overline{\text { Dahm+ }}$ & $\begin{array}{l}\text { Ct. Rate. } \\
{\left[10^{-4} s^{-1}\right]}\end{array}$ \\
\hline 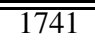 & 6:41:29.2 & 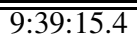 & $\overline{1.00}$ & - & - & $\overline{-}$ & $\overline{7078}$ & - & $\overline{-}$ & $\overline{-}$ & $\overline{<<8.9}$ \\
\hline 1742 & $6: 41: 29.3$ & $9: 30: 12.2$ & 1.00 & - & - & - & 7081 & - & - & - & $<2.6$ \\
\hline 1743 & $6: 41: 29.3$ & $9: 34: 52.3$ & 1.00 & - & - & - & 7082 & - & - & - & $<2.4$ \\
\hline 1744 & $6: 41: 29.3$ & 9:30:19.5 & 1.00 & - & - & - & 7084 & - & - & - & $<2.5$ \\
\hline 1745 & $6: 41: 29.3$ & $9: 28: 27.7$ & 0.62 & - & $06412927+0928276$ & - & 7086 & - & - & - & $<3.1$ \\
\hline 1746 & $6: 41: 29.3$ & $9: 34: 26.8$ & 1.00 & - & - & - & 7087 & - & - & - & $<2.1$ \\
\hline 1747 & $6: 41: 29.3$ & $9: 31: 10.1$ & 1.00 & - & - & - & 7089 & - & - & - & $<2.4$ \\
\hline 1748 & $6: 41: 29.3$ & $9: 29: 53.4$ & 0.50 & - & $06412933+0929534$ & 4082 & 7091 & - & - & - & $<2.6$ \\
\hline 1749 & $6: 41: 29.4$ & $9: 31: 58.3$ & 1.00 & - & - & - & 7093 & - & - & - & $<2.3$ \\
\hline 1750 & 6:41:29.4 & 9:36:22.7 & 1.00 & - & - & - & 7098 & - & - & - & $<2.3$ \\
\hline 1751 & $6: 41: 29.4$ & $9: 32: 03.1$ & 0.59 & - & $06412943+0932030$ & 4083 & 7097 & - & - & - & $<2.3$ \\
\hline $1752 *$ & $6: 41: 29.4$ & 9:39:18.2 & 0.50 & 413 & $06412944+0939181$ & 4084 & 7103 & $152 \dagger$ & - & - & 14.4 \\
\hline 1753 & $6: 41: 29.4$ & $9: 32: 51.3$ & 1.00 & - & - & - & 7100 & - & - & - & $<3.3$ \\
\hline 1754 & $6: 41: 29.4$ & $9: 41: 23.7$ & 1.00 & - & - & - & 7101 & - & - & - & $<4.9$ \\
\hline 1755 & $6: 41: 29.5$ & $9: 39: 48.4$ & 0.69 & - & $06412945+0939483$ & - & 7099 & - & - & - & $<4.9$ \\
\hline 1756 & $6: 41: 29.5$ & $9: 32: 13.3$ & 0.77 & - & $06412952+0932132$ & - & 7109 & - & - & - & $<2.3$ \\
\hline 1757 & $6: 41: 29.5$ & $9: 26: 44.7$ & 0.50 & - & $06412954+0926447$ & - & 7108 & - & - & - & $<6.4$ \\
\hline 1758 & 6:41:29.6 & $9: 40: 22.4$ & 1.00 & - & - & - & 7111 & - & - & - & $<3.2$ \\
\hline 1759 & 6:41:29.6 & $9: 29: 13.0$ & 0.50 & - & $06412957+0929129$ & - & 7114 & - & - & - & $<2.7$ \\
\hline 1760 & $6: 41: 29.7$ & 9:33:58.6 & 1.00 & - & - & - & 7118 & - & - & - & $<2.2$ \\
\hline 1761 & $6: 41: 29.7$ & 9:40:05.6 & 1.00 & - & - & - & 7119 & - & - & - & $<3.7$ \\
\hline 1762 & $6: 41: 29.7$ & 9:33:04.4 & 0.61 & - & $06412969+0933044$ & - & 7123 & - & - & - & $<4.3$ \\
\hline 1763 & $6: 41: 29.8$ & $9: 38: 42.2$ & 1.00 & - & - & - & 7130 & - & - & - & $<3.6$ \\
\hline 1764 & $6: 41: 29.9$ & $9: 31: 11.2$ & 1.00 & - & - & - & 7136 & - & - & - & $<2.4$ \\
\hline 1765 & $6: 41: 29.9$ & $9: 37: 14.5$ & 1.00 & - & - & - & 7137 & - & - & - & $<2.6$ \\
\hline 1766 & $6: 41: 29.9$ & 9:34:38.9 & 1.00 & - & - & - & 7138 & - & - & - & $<2.2$ \\
\hline 1767 & $6: 41: 30.0$ & $9: 39: 13.5$ & 0.50 & - & $06412995+0939135$ & - & 7141 & $152 \dagger$ & - & - & $<8.7$ \\
\hline 1768 & $6: 41: 30.0$ & 9:41:06.7 & 1.00 & - & - & - & 7143 & - & - & - & $<3.4$ \\
\hline 1769 & $6: 41: 30.0$ & $9: 36: 45.5$ & 1.00 & - & - & - & 7145 & - & - & - & $<2.5$ \\
\hline 1770 & $6: 41: 30.0$ & 9:38:30.9 & 1.00 & - & - & - & 7146 & - & - & - & $<3.3$ \\
\hline 1771 & $6: 41: 30.0$ & $9: 34: 39.5$ & 0.50 & - & $06413004+0934394$ & - & 7150 & - & - & - & $<2.3$ \\
\hline 1772 & $6: 41: 30.1$ & 9:33:13.4 & 1.00 & - & - & - & 7148 & - & - & - & $<2.6$ \\
\hline 1773 & $6: 41: 30.1$ & 9:37:02.1 & 0.52 & - & $06413007+0937020$ & 4096 & 7152 & - & - & - & $<2.7$ \\
\hline 1774 & $6: 41: 30.1$ & 9:39:08.4 & 1.00 & - & - & - & 7151 & - & - & - & $<4.0$ \\
\hline 1775 & $6: 41: 30.1$ & 9:31:00.6 & 1.00 & - & - & - & 7155 & - & - & - & $<2.5$ \\
\hline 1776 & $6: 41: 30.1$ & 9:34:11.1 & 1.00 & - & - & - & 7156 & - & - & - & $<2.3$ \\
\hline 1777 & $6: 41: 30.1$ & $9: 31: 45.8$ & 0.50 & - & $06413014+0931458$ & 4097 & 7163 & - & - & - & $<2.5$ \\
\hline 1778 & $6: 41: 30.2$ & $9: 28: 38.8$ & 1.00 & - & - & - & 7165 & - & - & - & $<5.8$ \\
\hline 1779 & $6: 41: 30.3$ & 9:32:06.6 & 1.00 & - & - & - & 7169 & - & - & - & $<2.5$ \\
\hline 1780 & $6: 41: 30.3$ & $9: 32: 42.5$ & 1.00 & - & - & - & 7170 & - & - & - & $<2.5$ \\
\hline 1781 & $6: 41: 30.3$ & $9: 33: 52.2$ & 1.00 & - & - & - & 7173 & - & - & - & $<2.3$ \\
\hline 1782 & $6: 41: 30.4$ & $9: 38: 24.7$ & 1.00 & - & - & - & 7177 & - & - & - & $<3.3$ \\
\hline 1783 & $6: 41: 30.4$ & $9: 38: 30.1$ & 0.50 & - & $06413038+0938300$ & 4104 & 7180 & - & - & - & $<3.4$ \\
\hline 1784 & $6: 41: 30.4$ & $9: 36: 56.6$ & 1.00 & - & - & - & 7179 & - & - & - & $<2.7$ \\
\hline 1785 & $6: 41: 30.4$ & $9: 28: 41.5$ & 1.00 & - & - & - & 7181 & - & - & - & $<5.9$ \\
\hline 1786 & $6: 41: 30.5$ & $9: 38: 05.8$ & 1.00 & - & - & - & 7187 & - & - & - & $<3.1$ \\
\hline 1787 & $6: 41: 30.5$ & $9: 30: 55.8$ & 0.71 & - & $06413050+0930557$ & 4105 & 7184 & - & - & - & $<2.6$ \\
\hline 1788 & $6: 41: 30.5$ & 9:36:07.1 & 1.00 & - & - & - & 7188 & - & - & - & $<2.3$ \\
\hline 1789 & $6: 41: 30.6$ & $9: 28: 18.4$ & 0.50 & - & $06413058+0928184$ & 4106 & 7191 & - & - & - & $<13.7$ \\
\hline 1790 & $6: 41: 30.6$ & $9: 30: 30.3$ & 1.00 & - & - & - & 7192 & - & - & - & $<2.7$ \\
\hline 1791 & 6:41:30.6 & $9: 30: 48.4$ & 1.00 & - & - & - & 7193 & - & - & - & $<2.7$ \\
\hline 1792 & $6: 41: 30.6$ & $9: 40: 40.0$ & 1.00 & - & - & - & 7197 & - & - & - & $<3.3$ \\
\hline 1793 & $6: 41: 30.7$ & $9: 37: 12.9$ & 1.00 & - & - & - & 7199 & - & - & - & $<2.8$ \\
\hline 1794 & $6: 41: 30.7$ & $9: 41: 24.7$ & 1.00 & - & - & - & 7200 & - & - & - & $<6.3$ \\
\hline 1795 & $6: 41: 30.7$ & 9:38:32.0 & 1.00 & - & - & - & 7202 & - & - & - & $<3.5$ \\
\hline 1796 & $6: 41: 30.7$ & 9:31:09.9 & 0.50 & - & $06413070+0931098$ & - & 7203 & - & - & - & $<2.5$ \\
\hline 1797 & 6:41:30.7 & 9:32:14.4 & 1.00 & - & - & - & 7205 & - & - & - & $<2.6$ \\
\hline 1798 & $6: 41: 30.7$ & $9: 32: 16.0$ & 1.00 & - & - & - & 7207 & - & - & - & $<2.6$ \\
\hline 1799 & $6: 41: 30.8$ & $9: 33: 45.1$ & 1.00 & - & - & - & 7210 & - & - & - & $<2.3$ \\
\hline 1800 & $6: 41: 30.8$ & $9: 29: 50.2$ & 1.00 & - & - & - & 7211 & - & - & - & $<2.9$ \\
\hline
\end{tabular}


Table 3. (Continued)

\begin{tabular}{|c|c|c|c|c|c|c|c|c|c|c|c|}
\hline$\overline{\mathrm{N}}$ & $\begin{array}{c}\mathrm{RA} \\
{[\mathrm{h} \mathrm{m} \mathrm{s}]}\end{array}$ & $\begin{array}{c}\text { Dec. } \\
{[\mathrm{d} \mathrm{m} \mathrm{s}]}\end{array}$ & $\begin{array}{c}\text { Id. rad. } \\
\text { ["] }\end{array}$ & ACIS & 2Mass & $\overline{\text { Reb.+ }}$ & $\overline{\text { Lamm+ }}$ & Flacc+ & $\overline{\text { W56 }}$ & $\overline{\mathrm{Dahm+}}$ & $\begin{array}{l}\text { Ct. Rate. } \\
{\left[10^{-4} s^{-1}\right]}\end{array}$ \\
\hline 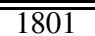 & 6 6:41:30.8 & $99: 32: 47.4$ & $\overline{0.50}$ & $\overline{-1}$ & "06413077+0932474 & 84109 & $\overline{7215}$ & $\overline{-1}$ & $\overline{-1}$ & 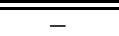 & (<3.2 \\
\hline 1802 & $6: 41: 30.8$ & 9:30:08.4 & 0.50 & - & $06413078+0930083$ & 4110 & 7214 & - & - & - & $<2.8$ \\
\hline $1803 *$ & $6: 41: 30.8$ & 9:39:00.7 & 3.08 & 414 & - & - & - & - & - & - & 13.7 \\
\hline 1804 & $6: 41: 30.9$ & $9: 30: 25.0$ & 0.72 & - & $06413088+0930250$ & - & 7222 & - & - & - & $<2.8$ \\
\hline $1805^{*}$ & $6: 41: 31.0$ & $9: 35: 25.3$ & 2.75 & 415 & - & - & - & - & - & - & 5.8 \\
\hline 1806 & $6: 41: 31.0$ & $9: 41: 22.6$ & 1.00 & - & - & - & 7228 & - & - & - & $<5.9$ \\
\hline 1807 & $6: 41: 31.1$ & 9:33:44.9 & 1.00 & - & - & - & 7232 & - & - & - & $<2.3$ \\
\hline 1808 & $6: 41: 31.1$ & $9: 32: 14.8$ & 0.54 & - & $06413109+0932148$ & - & 7239 & - & - & - & $<2.6$ \\
\hline 1809 & $6: 41: 31.2$ & $9: 29: 39.6$ & 0.50 & - & $06413123+0929396$ & - & 7256 & - & - & - & $<3.6$ \\
\hline 1810 & $6: 41: 31.2$ & $9: 34: 27.6$ & 0.65 & - & $06413123+0934275$ & - & 7250 & - & - & - & $<2.4$ \\
\hline 1811 & $6: 41: 31.3$ & $9: 29: 16.4$ & 1.00 & - & - & - & 7255 & - & - & - & $<3.5$ \\
\hline 1812 & $6: 41: 31.3$ & 9:37:03.2 & 1.00 & - & - & - & 7261 & - & - & - & $<2.9$ \\
\hline $1813 *$ & $6: 41: 31.3$ & $9: 32: 57.3$ & 1.00 & - & - & - & - & - & - & 466 & $<4.6$ \\
\hline 1814 & $6: 41: 31.3$ & $9: 31: 16.4$ & 1.00 & - & - & - & 7264 & - & - & - & $<2.5$ \\
\hline 1815 & $6: 41: 31.3$ & 9:41:05.3 & 1.00 & - & - & - & 7265 & - & - & - & $<3.5$ \\
\hline 1816 & $6: 41: 31.3$ & $9: 40: 54.2$ & 1.00 & - & - & - & 7267 & - & - & - & $<3.4$ \\
\hline 1817 & $6: 41: 31.3$ & $9: 35: 12.0$ & 0.50 & - & $06413132+0935120$ & - & 7272 & - & - & - & $<2.6$ \\
\hline 1818 & $6: 41: 31.3$ & 9:33:14.0 & 1.00 & - & - & - & 7270 & - & - & - & $<2.6$ \\
\hline 1819 & $6: 41: 31.3$ & $9: 40: 35.9$ & 1.00 & - & - & - & 7271 & - & - & - & $<3.3$ \\
\hline 1820 & $6: 41: 31.3$ & 9:40:03.8 & 1.00 & - & - & 4123 & 7274 & - & - & - & $<3.3$ \\
\hline 1821 & $6: 41: 31.3$ & 9:38:43.6 & 1.00 & - & - & - & 7273 & - & - & - & $<3.6$ \\
\hline 1822 & $6: 41: 31.4$ & 9:32:42.9 & 1.00 & - & - & - & 7275 & - & - & - & $<2.9$ \\
\hline 1823 & $6: 41: 31.4$ & $9: 40: 39.5$ & 1.00 & - & - & - & 7276 & - & - & - & $<3.2$ \\
\hline 1824 & $6: 41: 31.4$ & $9: 35: 28.3$ & 0.50 & - & $06413136+0935282$ & 4124 & 7279 & - & - & - & $<4.5$ \\
\hline 1825 & $6: 41: 31.4$ & $9: 38: 50.5$ & 0.50 & - & $06413141+0938504$ & 4126 & 7281 & - & - & - & $<3.7$ \\
\hline 1826 & $6: 41: 31.5$ & $9: 36: 50.5$ & 0.50 & - & $06413146+0936505$ & 4128 & 7288 & - & - & - & $<2.8$ \\
\hline 1827 & $6: 41: 31.5$ & 9:39:44.6 & 1.00 & - & - & - & 7285 & - & - & - & $<3.5$ \\
\hline 1828 & $6: 41: 31.5$ & $9: 32: 22.8$ & 0.74 & - & $06413150+0932228$ & - & 7292 & - & - & - & $<2.5$ \\
\hline 1829 & $6: 41: 31.5$ & 9:34:39.7 & 1.00 & - & - & - & 7291 & - & - & - & $<2.4$ \\
\hline 1830 & $6: 41: 31.6$ & 9:39:29.6 & 0.69 & - & $06413156+0939296$ & 4130 & 7294 & - & - & - & $<3.4$ \\
\hline 1831 & $6: 41: 31.6$ & $9: 36: 12.7$ & 1.00 & - & - & - & 7295 & - & - & - & $<2.4$ \\
\hline 1832 & $6: 41: 31.6$ & $9: 36: 53.2$ & 1.00 & - & - & - & 7296 & - & - & - & $<2.8$ \\
\hline 1833 & $6: 41: 31.6$ & $9: 30: 24.7$ & 1.00 & - & - & - & 7302 & - & - & - & $<2.9$ \\
\hline $1834 *$ & $6: 41: 31.7$ & $9: 31: 54.3$ & 0.50 & 416 & $06413168+0931543$ & 4133 & 7303 & - & - & - & 5.9 \\
\hline 1835 & $6: 41: 31.7$ & 9:30:00.6 & 0.50 & - & $06413171+0930006$ & 4134 & 7305 & - & - & - & $<8.4$ \\
\hline 1836 & $6: 41: 31.7$ & $9: 39: 42.2$ & 1.00 & - & - & - & 7306 & - & - & - & $<3.3$ \\
\hline 1837 & $6: 41: 32.0$ & $9: 36: 46.9$ & 0.50 & - & $06413197+0936468$ & 4141 & 7328 & - & - & - & $<2.9$ \\
\hline 1838 & $6: 41: 32.1$ & $9: 34: 40.3$ & 1.00 & - & - & - & 7337 & - & - & - & $<2.5$ \\
\hline 1839 & $6: 41: 32.1$ & $9: 32: 25.1$ & 0.50 & - & $06413205+0932251$ & - & 7341 & - & - & - & $<2.5$ \\
\hline 1840 & $6: 41: 32.1$ & $9: 36: 15.5$ & 1.00 & - & - & - & 7338 & - & - & - & $<2.6$ \\
\hline 1841 & $6: 41: 32.1$ & $9: 37: 57.0$ & 1.00 & - & - & - & 7342 & - & - & - & $<18.8$ \\
\hline 1842 & $6: 41: 32.1$ & $9: 30: 02.7$ & 1.00 & - & - & - & 7343 & - & - & - & $<17.3$ \\
\hline 1843 & $6: 41: 32.1$ & $9: 34: 30.1$ & 1.00 & - & - & - & 7344 & - & - & - & $<2.5$ \\
\hline 1844 & $6: 41: 32.1$ & $9: 37: 51.0$ & 1.00 & - & - & - & 7346 & - & - & - & $<3.4$ \\
\hline 1845 & $6: 41: 32.1$ & $9: 31: 19.8$ & 0.83 & - & $06413214+0931197$ & - & - & - & - & - & $<2.6$ \\
\hline 1846 & $6: 41: 32.2$ & $9: 37: 42.9$ & 0.65 & - & $06413216+0937428$ & - & - & - & - & - & $<3.2$ \\
\hline 1847 & $6: 41: 32.2$ & $9: 39: 53.1$ & 0.50 & - & $06413220+0939530$ & 4149 & - & - & - & - & $<3.6$ \\
\hline 1848 & $6: 41: 32.2$ & $9: 39: 25.6$ & 0.50 & - & $06413223+0939256$ & 4151 & - & - & - & - & $<3.4$ \\
\hline 1849 & $6: 41: 32.5$ & $9: 37: 16.3$ & 0.50 & - & $06413245+0937163$ & 4153 & - & - & - & - & $<2.9$ \\
\hline $1850 *$ & $6: 41: 32.5$ & 9:38:07.4 & 0.50 & 417 & $06413250+0938074$ & 4155 & - & 156 & - & - & 64.4 \\
\hline 1851 & $6: 41: 32.5$ & $9: 33: 10.5$ & 0.50 & - & $06413251+0933104$ & 4156 & - & - & - & - & $<3.4$ \\
\hline 1852 & $6: 41: 32.7$ & $9: 35: 02.2$ & 0.50 & - & $06413272+0935021$ & - & - & - & - & - & $<2.6$ \\
\hline 1853 & $6: 41: 32.8$ & $9: 40: 32.5$ & 0.54 & - & $06413278+0940324$ & 4165 & - & - & - & - & $<3.5$ \\
\hline 1854 & $6: 41: 32.9$ & 9:32:12.1 & 0.50 & - & $06413289+0932121$ & 4168 & - & - & - & - & $<2.9$ \\
\hline 1855 & $6: 41: 33.2$ & 9:36:07.8 & 0.63 & - & $06413323+0936077$ & - & - & - & - & - & $<2.7$ \\
\hline 1856 & $6: 41: 33.4$ & $9: 40: 12.8$ & 0.50 & - & $06413341+0940128$ & 4174 & - & - & 219 & - & $<3.6$ \\
\hline 1857 & $6: 41: 33.7$ & 9:36:02.2 & 0.50 & - & $06413372+0936022$ & - & - & - & - & - & $<2.8$ \\
\hline 1858 & $6: 41: 33.7$ & $9: 36: 14.4$ & 0.50 & - & $06413372+0936143$ & - & - & - & - & - & $<3.0$ \\
\hline 1859 & $6: 41: 33.8$ & $9: 35: 21.5$ & 0.50 & - & $06413375+0935214$ & - & - & - & - & - & $<3.2$ \\
\hline 1860 & $6: 41: 33.8$ & $9: 41: 12.4$ & 0.50 & - & $06413379+0941123$ & 4181 & - & - & - & - & $<6.7$ \\
\hline
\end{tabular}


Table 3. (Continued)

\begin{tabular}{|c|c|c|c|c|c|c|c|c|c|c|c|}
\hline$\overline{\mathrm{N}}$ & $\begin{array}{c}\mathrm{RA} \\
{[\mathrm{h} \mathrm{m} \mathrm{s}]}\end{array}$ & $\begin{array}{c}\text { Dec. } \\
{[\mathrm{d} \mathrm{m} \mathrm{s}]}\end{array}$ & $\begin{array}{c}\text { Id. rad. } \\
\text { ["] }\end{array}$ & $\overline{\text { ACIS }}$ & 2Mass & Reb.+ & $\overline{\text { Lamm+ }}$ & Flacc+ & $\overline{\text { W56 }}$ & Dahm+ & $\begin{array}{l}\text { Ct. Rate. } \\
{\left[10^{-4} s^{-1}\right]}\end{array}$ \\
\hline$\overline{1861}$ & $6: 41: 33.9$ & $\overline{\overline{9: 32: 45.4}}$ & $\overline{\overline{1.00}}$ & 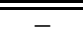 & $\overline{-1}$ & $\overline{-1}$ & $\overline{7364}$ & $\overline{-1}$ & $\overline{-1}$ & $\overline{-1}$ & 安8.7 \\
\hline 1862 & $6: 41: 33.9$ & $9: 38: 48.3$ & 0.50 & - & $06413390+0938482$ & 4184 & - & - & - & - & $<3.6$ \\
\hline 1863 & $6: 41: 34.0$ & $9: 39: 46.8$ & 1.00 & - & - & - & 7372 & - & - & - & $<4.0$ \\
\hline 1864 & $6: 41: 34.0$ & $9: 39: 15.5$ & 1.00 & - & - & 4190 & 7374 & - & - & - & $<3.8$ \\
\hline 1865 & $6: 41: 34.1$ & $9: 34: 24.4$ & 1.00 & - & - & - & 7376 & - & - & - & $<3.1$ \\
\hline 1866 & $6: 41: 34.2$ & 9:36:08.5 & 0.50 & - & $06413416+0936084$ & 4194 & 7382 & - & - & - & $<3.2$ \\
\hline 1867 & $6: 41: 34.2$ & $9: 36: 49.3$ & 1.00 & - & - & - & 7384 & - & - & - & $<3.3$ \\
\hline 1868 & $6: 41: 34.2$ & 9:35:07.1 & 0.50 & - & $06413420+0935071$ & - & 7387 & - & - & - & $<3.4$ \\
\hline 1869 & $6: 41: 34.2$ & $9: 33: 45.8$ & 1.00 & - & - & - & 7391 & - & - & - & $<3.1$ \\
\hline 1870 & $6: 41: 34.3$ & $9: 34: 42.4$ & 0.50 & - & $06413426+0934423$ & 4197 & 7393 & - & - & - & $<3.2$ \\
\hline 1871 & $6: 41: 34.3$ & $9: 38: 52.5$ & 0.62 & - & $06413431+0938525$ & - & - & - & - & - & $<3.6$ \\
\hline 1872 & $6: 41: 34.4$ & 9:39:09.7 & 1.00 & - & - & - & 7402 & - & - & - & $<4.0$ \\
\hline 1873 & $6: 41: 34.4$ & 9:39:51.6 & 1.00 & - & - & - & 7403 & - & - & - & $<4.2$ \\
\hline 1874 & $6: 41: 34.4$ & $9: 41: 04.2$ & 1.00 & - & - & - & 7405 & - & - & - & $<4.6$ \\
\hline 1875 & $6: 41: 34.5$ & $9: 35: 41.5$ & 0.50 & - & $06413447+0935414$ & - & 7411 & - & - & - & $<3.6$ \\
\hline 1876 & $6: 41: 34.5$ & $9: 39: 49.6$ & 1.00 & - & - & - & 7412 & - & - & - & $<4.2$ \\
\hline 1877 & $6: 41: 34.5$ & 9:36:11.7 & 1.00 & - & - & - & 7417 & - & - & - & $<3.7$ \\
\hline $1878^{*}$ & $6: 41: 34.6$ & $9: 36: 32.5$ & 0.50 & 418 & $06413455+0936325$ & 4204 & 7418 & - & - & 470 & 11.5 \\
\hline 1879 & $6: 41: 34.6$ & $9: 34: 46.5$ & 0.50 & - & $06413455+0934465$ & - & 7422 & - & - & - & $<3.2$ \\
\hline 1880 & 6:41:34.6 & $9: 33: 45.6$ & 0.50 & - & $06413456+0933455$ & - & 7421 & - & - & - & $<3.2$ \\
\hline 1881 & $6: 41: 34.6$ & $9: 37: 16.1$ & 1.00 & - & - & - & 7432 & - & - & - & $<3.0$ \\
\hline 1882 & $6: 41: 34.7$ & $9: 40: 38.4$ & 1.00 & - & - & - & 7434 & - & - & - & $<3.9$ \\
\hline 1883 & $6: 41: 34.7$ & $9: 39: 18.2$ & 1.00 & - & - & - & 7438 & - & - & - & $<4.1$ \\
\hline 1884 & $6: 41: 34.8$ & $9: 41: 02.5$ & 1.00 & - & - & - & 7441 & - & - & - & $<9.1$ \\
\hline 1885 & $6: 41: 34.8$ & $9: 33: 59.3$ & 1.00 & - & - & - & 7442 & - & - & - & $<3.2$ \\
\hline 1886 & $6: 41: 34.9$ & $9: 35: 18.7$ & 0.50 & - & $06413485+0935187$ & - & 7452 & - & - & - & $<3.7$ \\
\hline 1887 & $6: 41: 34.9$ & $9: 40: 53.6$ & 1.00 & - & - & - & 7454 & - & - & - & $<4.3$ \\
\hline 1888 & $6: 41: 35.0$ & $9: 40: 50.7$ & 1.00 & - & - & - & 7460 & - & - & - & $<4.2$ \\
\hline 1889 & $6: 41: 35.0$ & $9: 37: 19.8$ & 1.00 & - & - & - & 7464 & - & - & - & $<3.3$ \\
\hline 1890 & $6: 41: 35.1$ & 9:39:06.5 & 0.50 & - & $06413506+0939065$ & 4222 & - & - & - & - & $<4.2$ \\
\hline 1891 & $6: 41: 35.1$ & $9: 40: 38.2$ & 0.50 & - & $06413513+0940382$ & - & 7473 & - & - & - & $<4.1$ \\
\hline 1892 & $6: 41: 35.2$ & 9:38:28.1 & 0.50 & - & $06413518+0938280$ & 4226 & 7478 & - & - & - & $<3.7$ \\
\hline 1893 & $6: 41: 35.2$ & $9: 40: 29.0$ & 1.00 & - & - & - & 7484 & - & - & - & $<4.3$ \\
\hline 1894 & $6: 41: 35.3$ & $9: 36: 55.3$ & 1.00 & - & - & - & 7490 & - & - & - & $<3.8$ \\
\hline 1895 & $6: 41: 35.3$ & $9: 34: 58.6$ & 1.00 & - & - & - & 7494 & - & - & - & $<4.3$ \\
\hline 1896 & $6: 41: 35.5$ & $9: 34: 41.7$ & 1.00 & - & - & - & 7508 & - & - & - & $<27.2$ \\
\hline 1897 & $6: 41: 35.5$ & $9: 40: 54.7$ & 1.00 & - & - & - & 7512 & - & - & - & $<7.0$ \\
\hline 1898 & $6: 41: 35.7$ & 9:38:53.3 & 0.66 & - & $06413570+0938533$ & - & - & - & - & - & $<4.1$ \\
\hline 1899 & $6: 41: 35.8$ & $9: 34: 50.1$ & 0.50 & - & $06413577+0934500$ & 4245 & 7546 & - & - & - & $<37.5$ \\
\hline 1900 & $6: 41: 35.8$ & $9: 40: 14.2$ & 1.00 & - & - & - & 7551 & - & - & - & $<4.2$ \\
\hline 1901 & $6: 41: 35.9$ & $9: 34: 40.4$ & 0.50 & - & $06413585+0934403$ & 4249 & 7554 & - & - & - & $<51.6$ \\
\hline 1902 & $6: 41: 35.9$ & $9: 40: 42.0$ & 0.50 & - & $06413589+0940419$ & - & 7557 & - & - & - & $<4.2$ \\
\hline 1903 & $6: 41: 35.9$ & $9: 40: 53.1$ & 1.00 & - & - & - & 7559 & - & - & - & $<6.6$ \\
\hline 1904 & $6: 41: 35.9$ & $9: 39: 46.3$ & 0.50 & - & $06413594+0939462$ & 4251 & 7564 & - & - & - & $<4.4$ \\
\hline 1905 & $6: 41: 36.0$ & 9:41:00.4 & 1.00 & - & - & - & 7568 & - & - & - & $<18.7$ \\
\hline 1906 & $6: 41: 36.0$ & $9: 37: 37.8$ & 1.00 & - & - & - & 7571 & - & - & - & $<3.9$ \\
\hline 1907 & $6: 41: 36.1$ & $9: 39: 41.5$ & 1.00 & - & - & - & 7580 & - & - & - & $<4.6$ \\
\hline 1908 & $6: 41: 36.1$ & $9: 40: 07.5$ & 1.00 & - & - & - & 7588 & - & - & - & $<4.2$ \\
\hline 1909 & $6: 41: 36.2$ & $9: 40: 45.1$ & 0.50 & - & $06413619+0940451 \dagger$ & 4260 & $7592 \dagger$ & - & - & - & $<4.5$ \\
\hline 1910 & $6: 41: 36.2$ & $9: 40: 45.1$ & 0.50 & - & $06413619+0940451 \dagger$ & 4261 & $7592 \dagger$ & - & - & - & $<4.5$ \\
\hline $1911^{*}$ & $6: 41: 36.2$ & $9: 39: 20.4$ & 0.50 & 419 & $06413623+0939204$ & 4263 & 7597 & - & - & 471 & 14.1 \\
\hline 1912 & $6: 41: 36.3$ & $9: 40: 15.3$ & 0.50 & - & $06413629+0940153$ & - & 7602 & - & - & - & $<4.3$ \\
\hline 1913 & $6: 41: 36.3$ & $9: 38: 48.0$ & 1.00 & - & - & - & 7606 & - & - & - & $<4.1$ \\
\hline 1914 & $6: 41: 36.4$ & 9:39:42.0 & 1.00 & - & - & 4270 & 7618 & - & - & - & $<4.7$ \\
\hline 1915 & $6: 41: 36.5$ & 9:39:07.2 & 1.00 & - & - & - & 7621 & - & - & - & $<10.6$ \\
\hline 1916 & $6: 41: 36.5$ & $9: 39: 17.0$ & 0.50 & - & $06413653+0939169$ & - & 7628 & - & - & - & $<11.7$ \\
\hline 1917 & $6: 41: 36.6$ & $9: 39: 18.7$ & 1.00 & - & - & - & 7630 & - & - & - & $<11.7$ \\
\hline 1918 & $6: 41: 36.6$ & $9: 37: 00.6$ & 0.56 & - & $06413657+0937006$ & - & 7629 & - & - & - & $<4.0$ \\
\hline 1919 & $6: 41: 36.6$ & $9: 37: 56.3$ & 0.50 & - & $06413660+0937563$ & 4275 & 7634 & - & - & - & $<4.1$ \\
\hline 1920 & $6: 41: 36.7$ & $9: 39: 27.5$ & 0.50 & - & $06413668+0939274$ & - & 7640 & - & - & - & $<11.7$ \\
\hline
\end{tabular}


Table 3. (Continued)

\begin{tabular}{|c|c|c|c|c|c|c|c|c|c|c|c|}
\hline$\overline{\mathrm{N}}$ & $\begin{array}{c}\text { RA } \\
{[\mathrm{h} \mathrm{m} \mathrm{s}]}\end{array}$ & $\begin{array}{c}\text { Dec. } \\
{[\mathrm{d} \mathrm{m} \mathrm{s}]}\end{array}$ & $\begin{array}{c}\text { Id. rad. } \\
\text { ["] }\end{array}$ & $\overline{\mathrm{ACIS}}$ & 2Mass & Reb.+ & Lamm+ & Flacc+ & W56 & $\overline{\text { Dahm+ }}$ & $\begin{array}{l}\text { Ct. Rate. } \\
{\left[10^{-4} s^{-1}\right]}\end{array}$ \\
\hline 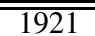 & $\overline{60: 41: 36.7}$ & $\overline{99: 38: 30.3}$ & $\overline{\overline{0.50}}$ & $=$ & $06413668+0938303$ & 424281 & $\overline{7641}$ & $=$ & $\overline{-}$ & $=$ & $\overline{<<4.1}$ \\
\hline 1922 & $6: 41: 36.8$ & $9: 40: 15.2$ & 1.00 & - & - & - & 7655 & - & - & - & $<4.3$ \\
\hline 1923 & $6: 41: 36.8$ & $9: 36: 36.8$ & 1.00 & - & - & - & 7656 & - & - & - & $<4.3$ \\
\hline 1924 & $6: 41: 36.8$ & $9: 40: 59.0$ & 1.00 & - & - & - & 7662 & - & - & - & $<25.4$ \\
\hline 1925 & $6: 41: 36.9$ & $9: 36: 12.0$ & 1.00 & - & - & - & 7671 & - & - & - & $<37.9$ \\
\hline 1926 & $6: 41: 36.9$ & $9: 40: 22.1$ & 1.00 & - & - & - & 7677 & - & - & - & $<4.4$ \\
\hline 1927 & $6: 41: 37.0$ & $9: 40: 27.7$ & 0.50 & - & $06413697+0940277$ & - & 7680 & - & - & - & $<4.3$ \\
\hline 1928 & $6: 41: 37.0$ & $9: 38: 13.7$ & 1.00 & - & - & - & 7687 & - & - & - & $<4.1$ \\
\hline 1929 & $6: 41: 37.1$ & $9: 37: 07.2$ & 0.50 & - & $06413712+0937072$ & 4298 & 7699 & - & - & - & $<4.7$ \\
\hline 1930 & $6: 41: 37.2$ & $9: 38: 14.0$ & 1.00 & - & - & - & 7705 & - & - & - & $<4.1$ \\
\hline 1931 & $6: 41: 37.3$ & $9: 39: 12.4$ & 0.76 & - & $06413727+0939124$ & - & 7710 & - & - & - & $<5.4$ \\
\hline 1932 & $6: 41: 37.3$ & $9: 36: 42.7$ & 1.00 & - & - & - & 7719 & - & - & - & $<5.2$ \\
\hline 1933 & $6: 41: 37.4$ & $9: 37: 28.1$ & 0.50 & - & $06413743+0937280$ & 4305 & 7732 & - & - & - & $<19.4$ \\
\hline 1934 & $6: 41: 37.4$ & $9: 37: 14.0$ & 1.00 & - & - & - & 7733 & - & - & - & $<8.9$ \\
\hline 1935 & $6: 41: 37.5$ & $9: 39: 25.8$ & 0.50 & - & $06413745+0939258$ & 4307 & 7734 & - & - & - & $<5.6$ \\
\hline 1936 & $6: 41: 37.5$ & $9: 40: 26.6$ & 1.00 & - & - & - & 7739 & - & - & - & $<5.0$ \\
\hline 1937 & $6: 41: 37.5$ & $9: 40: 58.2$ & 0.50 & - & $06413752+0940582$ & - & 7742 & - & - & - & $<24.1$ \\
\hline 1938 & $6: 41: 37.5$ & 9:40:40.8 & 0.50 & - & $06413754+0940407$ & 4309 & 7745 & - & - & - & $<5.2$ \\
\hline 1939 & $6: 41: 37.6$ & $9: 40: 29.8$ & 0.71 & - & $06413764+0940298$ & 4313 & 7759 & - & - & - & $<5.2$ \\
\hline 1940 & $6: 41: 37.8$ & $9: 37: 52.2$ & 1.00 & - & - & - & 7773 & - & - & - & $<5.4$ \\
\hline 1941 & $6: 41: 38.0$ & $9: 39: 53.4$ & 0.50 & - & $06413797+0939534$ & 4328 & 7783 & - & - & - & $<4.8$ \\
\hline 1942 & $6: 41: 38.4$ & $9: 39: 15.2$ & 1.00 & - & - & - & 7825 & - & - & - & $<9.4$ \\
\hline 1943 & $6: 41: 38.4$ & $9: 40: 47.6$ & 1.00 & - & - & - & 7826 & - & - & - & $<17.2$ \\
\hline 1944 & $6: 41: 38.4$ & $9: 38: 18.3$ & 1.00 & - & - & - & 7829 & - & - & - & $<5.0$ \\
\hline 1945 & $6: 41: 38.4$ & $9: 40: 59.2$ & 1.00 & - & - & - & 7837 & - & - & - & $<22.2$ \\
\hline 1946 & $6: 41: 38.5$ & $9: 39: 50.0$ & 1.00 & - & - & - & 7844 & - & - & - & $<4.8$ \\
\hline 1947 & $6: 41: 38.5$ & $9: 37: 56.3$ & 0.50 & - & $06413848+0937563$ & 4343 & 7842 & - & - & - & $<9.4$ \\
\hline 1948 & $6: 41: 38.5$ & $9: 39: 20.4$ & 0.50 & - & $06413849+0939203$ & 4344 & 7843 & - & - & - & $<6.9$ \\
\hline 1949 & $6: 41: 38.5$ & $9: 39: 38.6$ & 1.00 & - & - & - & 7846 & - & - & - & $<4.8$ \\
\hline 1950 & $6: 41: 38.5$ & $9: 40: 29.0$ & 0.75 & - & $06413852+0940290$ & - & 7847 & - & - & - & $<32.2$ \\
\hline 1951 & $6: 41: 38.7$ & $9: 38: 35.8$ & 0.54 & - & $06413866+0938358$ & 4346 & 7865 & - & - & - & $<22.2$ \\
\hline 1952 & $6: 41: 38.7$ & $9: 40: 10.3$ & 0.50 & - & $06413874+0940102$ & - & 7870 & - & - & - & $<9.7$ \\
\hline 1953 & $6: 41: 38.9$ & $9: 38: 52.1$ & 0.50 & - & $06413894+0938520$ & 4352 & - & - & - & - & $<4.6$ \\
\hline 1954 & $6: 41: 39.0$ & $9: 40: 20.7$ & 1.00 & - & - & - & 7895 & - & - & - & $<32.2$ \\
\hline 1955 & $6: 41: 39.1$ & $9: 39: 54.8$ & 1.00 & - & - & - & 7902 & - & - & - & $<6.1$ \\
\hline 1956 & $6: 41: 39.2$ & $9: 40: 13.2$ & 1.00 & - & - & - & 7922 & - & - & - & $<32.2$ \\
\hline 1957 & $6: 41: 39.4$ & $9: 39: 10.9$ & 1.00 & - & - & - & 7942 & - & - & - & $<35.8$ \\
\hline 1958 & $6: 41: 39.5$ & $9: 39: 22.9$ & 0.72 & - & $06413949+0939228$ & 4374 & 7952 & - & - & - & $<25.9$ \\
\hline 1959 & $6: 41: 39.5$ & $9: 39: 44.5$ & 1.00 & - & - & - & 7949 & - & - & - & $<5.4$ \\
\hline 1960 & $6: 41: 39.5$ & $9: 40: 09.5$ & 1.00 & - & - & - & 7950 & - & - & - & $<40.2$ \\
\hline 1961 & $6: 41: 39.6$ & $9: 40: 12.1$ & 1.00 & - & - & - & 7962 & - & - & - & $<44.2$ \\
\hline $1962 *$ & $6: 41: 39.8$ & $9: 40: 27.9$ & 0.50 & 420 & $06413974+0940279$ & 4382 & 7974 & 159 & - & 475 & 38.5 \\
\hline 1963 & $6: 41: 40.0$ & $9: 40: 55.5$ & 1.00 & - & - & - & 8001 & - & - & - & $<25.2$ \\
\hline 1964 & $6: 41: 40.1$ & $9: 40: 15.5$ & 1.00 & - & - & - & 8012 & - & - & - & $<32.2$ \\
\hline 1965 & $6: 41: 40.2$ & $9: 40: 39.0$ & 0.64 & - & $06414024+0940389$ & - & 8027 & - & - & - & $<32.2$ \\
\hline 1966 & $6: 41: 40.4$ & $9: 40: 46.9$ & 1.00 & - & - & - & 8036 & - & - & - & $<14.9$ \\
\hline 1967 & $6: 41: 40.7$ & $9: 40: 43.6$ & 0.79 & - & $06414070+0940435$ & - & 8070 & - & - & - & $<24.4$ \\
\hline
\end{tabular}

*: A likely NGC 2264 member;

$\dagger$ : Entry also present in another row. 
Table 4. Optical/NIR properties of X-ray sources.

\begin{tabular}{|c|c|c|c|c|c|c|c|c|c|c|c|c|c|}
\hline$\overline{\mathrm{N}}$ & 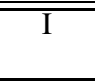 & "R-I & $\overline{\bar{J}}$ & $\overline{\mathrm{H}}$ & $\overline{\mathrm{K}}$ & Sp.T. & $\overline{\overline{\mathrm{Av}}}$ & $\overline{\mathrm{Av}(\mathrm{JHK})}$ & $\begin{array}{c}\log T_{e f f} \\
{[\mathrm{~K}]} \\
\end{array}$ & $\begin{array}{c}\log L_{b o l} \\
{\left[L_{\odot}\right]} \\
\end{array}$ & $\begin{array}{l}\text { Mass } \\
{\left[M_{\odot}\right]} \\
\end{array}$ & $\begin{array}{c}\begin{array}{c}\text { LogAge } \\
\text { [yr.] }\end{array} \\
\end{array}$ & $\begin{array}{l}P_{\text {rot }} \\
{[\mathrm{d}]} \\
\end{array}$ \\
\hline 1 & $\begin{array}{l}17.97 \\
\end{array}$ & 2.10 & 15.92 & 14.17 & 13.37 & - & - & 9.96 & - & - & - & - & - \\
\hline 2 & 15.68 & 1.25 & 14.27 & 13.54 & 13.29 & M2.5 & 0.00 & 0.62 & 3.54 & -0.80 & 0.34 & 6.63 & 1.23 \\
\hline 3 & 15.88 & 1.33 & 14.44 & 13.70 & 13.50 & M3 & 0.00 & 0.00 & 3.53 & -0.87 & 0.30 & 6.63 & 12.09 \\
\hline 4 & 14.14 & 1.03 & 12.79 & 12.07 & 11.85 & M0 & 0.62 & 0.41 & 3.59 & -0.06 & 0.57 & 6.11 & 4.55 \\
\hline 5 & 13.26 & 0.86 & 11.81 & 11.30 & 10.75 & K4 & 1.61 & 0.00 & 3.66 & 0.51 & 1.57 & 6.19 & 7.49 \\
\hline 6 & 15.15 & 1.33 & 13.66 & 12.97 & 12.78 & M3 & 0.00 & 0.00 & 3.53 & -0.57 & 0.32 & 6.33 & 0.76 \\
\hline 7 & 19.86 & 1.74 & - & - & - & - & - & - & - & - & - & - & - \\
\hline 8 & 14.39 & 0.86 & 12.69 & 11.51 & 10.67 & K1 & 1.92 & 2.39 & 3.71 & 0.15 & 1.30 & 7.10 & 12.09 \\
\hline 9 & 13.82 & 0.83 & 12.57 & 11.90 & 11.62 & K6 & 0.58 & 0.00 & 3.62 & 0.01 & 0.92 & 6.38 & 1.98 \\
\hline 10 & 17.06 & 1.70 & 15.42 & 14.85 & 14.60 & - & - & 0.00 & - & - & - & - & - \\
\hline 11 & 14.74 & 1.30 & 13.15 & 12.41 & 12.17 & - & - & 0.63 & - & - & - & - & 12.58 \\
\hline 12 & - & - & - & - & - & - & - & - & - & - & - & - & - \\
\hline 13 & 21.35 & - & - & - & - & - & - & - & - & - & - & - & - \\
\hline 14 & 15.92 & 1.27 & 14.51 & 13.79 & 13.57 & M3 & 0.00 & 0.00 & 3.53 & -0.88 & 0.30 & 6.65 & 0.76 \\
\hline 15 & 16.02 & 1.48 & 14.41 & 13.73 & 13.47 & - & - & 0.00 & - & - & - & - & 16.69 \\
\hline 16 & - & - & - & - & - & - & - & - & - & - & - & - & - \\
\hline 17 & 15.00 & 1.26 & 13.55 & 12.82 & 12.53 & M2 & 0.45 & 0.85 & 3.55 & -0.43 & 0.39 & 6.28 & 3.88 \\
\hline 18 & - & - & - & - & - & - & - & - & - & - & - & - & - \\
\hline 19 & 16.25 & 1.55 & 14.57 & - & 13.64 & - & - & - & - & - & - & - & - \\
\hline 20 & 16.10 & 1.76 & 14.51 & 13.98 & 13.67 & - & - & 0.00 & - & - & - & - & - \\
\hline 21 & - & - & - & - & - & - & - & - & - & - & - & - & - \\
\hline 22 & - & - & - & - & - & - & - & - & - & - & - & - & - \\
\hline 23 & - & - & - & - & - & - & - & - & - & - & - & - & - \\
\hline 24 & - & - & - & - & - & - & - & - & - & - & - & - & - \\
\hline 25 & 15.76 & 1.21 & 13.88 & 13.02 & 12.59 & M1 & 0.85 & 2.45 & 3.57 & -0.65 & 0.47 & 6.69 & - \\
\hline 26 & 14.81 & 1.35 & 13.33 & 12.55 & 12.33 & - & - & 1.00 & - & - & - & - & 1.15 \\
\hline 27 & 15.07 & 0.86 & 14.00 & 13.27 & 13.14 & K7 & 0.45 & 0.00 & 3.61 & -0.53 & 0.81 & 7.07 & 5.82 \\
\hline 28 & - & - & - & - & - & - & - & - & - & - & - & - & - \\
\hline 29 & 15.09 & 1.12 & - & - & - & - & - & - & - & - & - & - & - \\
\hline 30 & 14.45 & 1.18 & 12.91 & 12.19 & 11.83 & M3 & 0.00 & 0.00 & 3.53 & -0.29 & 0.32 & 6.12 & - \\
\hline 31 & - & - & - & - & - & - & - & - & - & - & - & - & - \\
\hline 32 & 15.23 & 1.11 & 13.93 & 13.19 & 12.89 & M1 & 0.40 & 0.98 & 3.57 & -0.54 & 0.48 & 6.57 & - \\
\hline 33 & 14.96 & 1.35 & 13.43 & 12.81 & 12.55 & M3 & 0.00 & 0.00 & 3.53 & -0.50 & 0.32 & 6.27 & 1.32 \\
\hline 34 & 16.60 & 2.00 & 14.63 & 14.01 & 13.58 & - & - & 0.00 & - & - & - & - & 1.18 \\
\hline 35 & - & - & - & - & - & - & - & - & - & - & - & - & - \\
\hline 36 & 14.56 & 0.92 & - & 12.56 & - & M0 & 0.13 & - & 3.59 & -0.35 & 0.58 & 6.47 & 4.55 \\
\hline 37 & - & - & - & - & - & - & - & - & - & - & - & - & - \\
\hline 38 & 14.50 & 1.26 & 12.65 & 11.14 & 9.95 & K6 & 2.50 & 4.26 & 3.62 & 0.21 & 0.91 & 6.11 & - \\
\hline 39 & 13.28 & 0.61 & 12.24 & 11.58 & 11.25 & K4 & 0.49 & 0.00 & 3.66 & 0.23 & 1.41 & 6.55 & 4.50 \\
\hline 40 & 17.28 & 1.75 & 15.61 & 15.02 & 14.75 & - & - & 0.00 & - & - & - & - & 1.89 \\
\hline 41 & 15.94 & 1.41 & - & - & - & - & - & - & - & - & - & - & 2.55 \\
\hline 42 & - & - & - & - & - & - & - & - & - & - & - & - & - \\
\hline 43 & 15.62 & 1.89 & - & 12.78 & - & - & - & - & - & - & - & - & 2.46 \\
\hline 44 & 17.70 & 2.03 & 15.61 & 15.13 & 14.84 & - & - & 0.00 & - & - & - & - & 0.62 \\
\hline 45 & 15.23 & 1.55 & 13.74 & 13.05 & 12.87 & M5 & 0.00 & 0.00 & 3.49 & -0.47 & 0.22 & 5.85 & 1.22 \\
\hline 46 & 14.66 & 0.83 & 13.12 & 12.25 & 11.45 & K7: & - & 0.00 & - & - & - & - & - \\
\hline 47 & 18.60 & 2.50 & - & - & - & - & - & - & - & - & - & - & - \\
\hline 48 & - & - & - & - & - & - & - & - & - & - & - & - & - \\
\hline 49 & 17.54 & 2.05 & 15.67 & 15.08 & 14.69 & M5 & 0.67 & 0.00 & 3.49 & -1.23 & 0.15 & 6.72 & 0.60 \\
\hline 50 & 15.90 & 1.43 & 14.35 & 13.66 & 13.46 & - & - & 0.00 & - & - & - & - & - \\
\hline 51 & 20.30 & 2.19 & 16.64 & 15.57 & 14.91 & - & - & 0.00 & - & - & - & - & - \\
\hline 52 & 11.43 & 0.31 & 10.76 & 10.28 & 9.84 & G0 & 0.00 & 0.00 & 3.78 & 0.88 & 1.67 & 6.97 & - \\
\hline 53 & 14.19 & 0.85 & 12.86 & 12.14 & 11.80 & M0 & 0.00 & 1.08 & 3.59 & -0.23 & 0.57 & 6.31 & 6.97 \\
\hline 54 & - & - & 8.25 & 8.34 & 8.37 & - & - & 0.00 & - & - & - & - & - \\
\hline 55 & 15.74 & 1.41 & 14.27 & 13.48 & 13.35 & - & - & 0.00 & - & - & - & - & 7.09 \\
\hline 56 & 14.17 & 0.63 & 13.23 & 12.64 & 12.52 & K4 & 0.58 & 0.00 & 3.66 & -0.10 & 1.17 & 7.02 & 14.81 \\
\hline 57 & 15.13 & 1.05 & - & 13.00 & 12.67 & K7 & 1.29 & - & 3.61 & -0.34 & 0.79 & 6.77 & 2.50 \\
\hline 58 & - & - & - & - & - & - & - & - & - & - & - & - & - \\
\hline 59 & 15.00 & 1.50 & 13.49 & 12.83 & 12.57 & M3 & 0.58 & 0.00 & 3.53 & -0.37 & 0.32 & 6.17 & 8.46 \\
\hline 60 & 15.55 & 1.29 & 13.97 & 13.11 & 12.39 & M0 & 1.78 & 0.00 & 3.59 & -0.34 & 0.58 & 6.45 & - \\
\hline
\end{tabular}


Table 4. (Continued)

\begin{tabular}{|c|c|c|c|c|c|c|c|c|c|c|c|c|c|}
\hline$\overline{\mathrm{N}}$ & 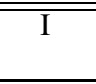 & R-I & $\overline{\bar{J}}$ & $\overline{\overline{\mathrm{H}}}$ & $\overline{\bar{K}}$ & $\overline{\text { Sp.T. }}$ & 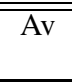 & 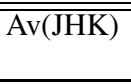 & $\begin{array}{c}\log T_{e f f} \\
{[\mathrm{~K}]}\end{array}$ & $\begin{array}{c}\log L_{b o l} \\
{\left[L_{\odot}\right]} \\
\end{array}$ & $\begin{array}{l}\text { Mass } \\
{\left[M_{\odot}\right]} \\
\end{array}$ & $\begin{array}{c}\begin{array}{c}\text { LogAge } \\
\text { [yr.] }\end{array} \\
\end{array}$ & $\begin{array}{l}P_{\text {rot }} \\
\text { [d] }\end{array}$ \\
\hline$\overline{61}$ & 14.54 & 1.10 & 13.23 & 12.50 & 12.36 & $\overline{\mathrm{M} 0}$ & 0.94 & $\overline{0.00}$ & 3.59 & -0.14 & 0.57 & $\overline{c 6.20}$ & 9.25 \\
\hline 62 & 16.51 & 1.86 & 14.65 & 14.03 & 13.80 & - & - & 0.00 & - & - & - & - & 0.55 \\
\hline 63 & - & - & - & - & - & - & - & - & - & - & - & - & - \\
\hline 64 & 16.84 & 1.86 & 14.82 & 14.02 & 13.88 & - & - & 0.00 & - & - & - & - & 1.96 \\
\hline 65 & 16.15 & 1.50 & 14.57 & 13.94 & 13.71 & - & - & 0.00 & - & - & - & - & 1.29 \\
\hline 66 & 16.32 & 1.80 & 14.47 & 13.89 & 13.50 & - & - & 0.00 & - & - & - & - & - \\
\hline 67 & - & - & - & - & - & - & - & - & - & - & - & - & - \\
\hline 68 & 15.62 & 1.42 & 14.05 & 13.34 & 12.98 & M3 & 0.22 & 0.00 & 3.53 & -0.71 & 0.31 & 6.46 & - \\
\hline 69 & - & - & - & - & - & - & - & - & - & - & - & - & - \\
\hline 70 & 17.43 & 2.44 & 14.90 & - & - & - & - & - & - & - & - & - & - \\
\hline 71 & 13.30 & 0.62 & 12.39 & 11.67 & 11.35 & K2 & 0.67 & 0.97 & 3.69 & 0.27 & 1.50 & 6.80 & - \\
\hline 72 & 15.17 & 1.94 & 13.22 & 12.61 & 12.34 & - & - & 0.00 & - & - & - & - & - \\
\hline 73 & - & - & - & - & - & - & - & - & - & - & - & - & - \\
\hline 74 & - & - & - & - & - & - & - & - & - & - & - & - & - \\
\hline 75 & 15.23 & 1.49 & 13.73 & 13.02 & 12.77 & M3.5 & 0.00 & 0.00 & 3.52 & -0.58 & 0.29 & 6.31 & 13.68 \\
\hline 76 & 20.45 & - & 16.28 & 14.41 & 13.50 & - & - & 11.24 & - & - & - & - & - \\
\hline 77 & 17.70 & 2.14 & 15.66 & 15.20 & 14.88 & - & - & 0.00 & - & - & - & - & 1.59 \\
\hline 78 & 14.10 & 0.94 & 12.77 & 12.00 & 11.69 & M1 & 0.00 & 1.19 & 3.57 & -0.19 & 0.47 & 6.13 & - \\
\hline 79 & 15.24 & 1.17 & 13.86 & 13.07 & 12.84 & M2 & 0.04 & 1.00 & 3.55 & -0.62 & 0.38 & 6.49 & 5.51 \\
\hline 80 & 16.35 & 1.74 & 14.53 & 13.38 & 12.61 & M4.5 & 0.00 & 2.60 & 3.50 & -0.96 & 0.20 & 6.55 & 9.71 \\
\hline 81 & 17.02 & 1.77 & 15.21 & 14.46 & 14.45 & - & - & 0.00 & - & - & - & - & 1.57 \\
\hline 82 & - & - & - & - & - & - & - & - & - & - & - & - & - \\
\hline 83 & 15.88 & 1.29 & 14.41 & 13.73 & 13.47 & - & - & 0.00 & - & - & - & - & 2.57 \\
\hline 84 & 17.37 & 2.04 & 15.32 & 14.82 & 14.37 & - & - & 0.00 & - & - & - & - & - \\
\hline 85 & 17.51 & 1.83 & 15.86 & 15.20 & 14.77 & - & - & 0.00 & - & - & - & - & - \\
\hline 86 & 15.30 & 1.47 & 13.88 & 13.14 & 12.93 & M5 & 0.00 & 0.51 & 3.49 & -0.50 & 0.22 & 5.99 & 3.09 \\
\hline 87 & 14.34 & 1.13 & 12.92 & 12.17 & 11.95 & M1 & 0.49 & 0.64 & 3.57 & -0.17 & 0.47 & 6.10 & 1.67 \\
\hline 88 & 16.45 & 1.51 & 14.95 & 14.23 & 13.94 & - & - & 0.00 & - & - & - & - & - \\
\hline 89 & 16.84 & 1.84 & 14.38 & 12.20 & 10.60 & - & - & 10.30 & - & - & - & - & - \\
\hline 90 & 14.99 & 0.97 & 13.69 & 13.04 & 12.80 & K8 & 0.65 & 0.00 & 3.60 & -0.42 & 0.70 & 6.74 & - \\
\hline 91 & 14.48 & 0.88 & 13.28 & 12.60 & 12.40 & K4 & 1.70 & 0.00 & 3.66 & 0.05 & 1.30 & 6.81 & 6.77 \\
\hline 92 & - & - & - & - & - & - & - & - & - & - & - & - & - \\
\hline 93 & 15.06 & 1.39 & 13.69 & 12.79 & 12.26 & - & - & 1.06 & - & - & - & - & - \\
\hline 94 & - & - & - & - & - & - & - & - & - & - & - & - & - \\
\hline 95 & 13.81 & 0.77 & 12.40 & 11.61 & 11.16 & K4 & 1.20 & 0.00 & 3.66 & 0.19 & 1.40 & 6.61 & 7.64 \\
\hline 96 & - & - & - & - & - & - & - & - & - & - & - & - & - \\
\hline 97 & 16.38 & 1.79 & 14.31 & 13.26 & 12.88 & M5 & 0.00 & 3.61 & 3.49 & -0.93 & 0.18 & 6.53 & 2.16 \\
\hline 98 & - & - & - & - & - & - & - & - & - & - & - & - & - \\
\hline 99 & 16.04 & 1.94 & 14.15 & 13.57 & 13.20 & - & - & 0.00 & - & - & - & - & - \\
\hline 100 & 16.15 & 1.65 & 14.35 & 13.78 & 13.56 & - & - & 0.00 & - & - & - & - & - \\
\hline 101 & - & - & - & - & - & - & - & - & - & - & - & - & - \\
\hline 102 & 13.20 & 0.63 & 12.36 & 11.62 & 11.16 & K4 & 0.58 & 0.00 & 3.66 & 0.28 & 1.48 & 6.49 & - \\
\hline 103 & 15.43 & 1.50 & 13.87 & 13.19 & 12.97 & M5 & 0.00 & 0.00 & 3.49 & -0.55 & 0.21 & 6.23 & - \\
\hline 104 & 14.97 & 0.94 & 13.72 & 12.99 & 12.87 & M1 & 0.00 & 0.00 & 3.57 & -0.54 & 0.48 & 6.56 & 9.04 \\
\hline 105 & 14.31 & 0.82 & 12.99 & 12.20 & 11.79 & K4 & 1.43 & 0.00 & 3.66 & 0.05 & 1.30 & 6.81 & - \\
\hline 106 & 16.21 & 1.63 & 14.15 & 13.34 & 12.85 & M4 & 0.00 & 0.00 & 3.51 & -0.94 & 0.23 & 6.60 & 7.22 \\
\hline 107 & - & - & - & - & - & - & - & - & - & - & - & - & - \\
\hline 108 & - & - & - & - & 13.61 & - & - & - & - & - & - & - & - \\
\hline 109 & 15.95 & 1.43 & 14.50 & 13.78 & 13.54 & - & - & 0.00 & - & - & - & - & - \\
\hline 110 & 17.81 & 1.80 & 15.94 & 15.24 & 14.92 & - & - & 0.00 & - & - & - & - & - \\
\hline 111 & 13.47 & 0.60 & 12.35 & 11.68 & 11.32 & K1 & 0.76 & 0.00 & 3.71 & 0.24 & 1.41 & 7.00 & 2.38 \\
\hline 112 & 11.14 & 0.31 & 10.61 & 10.34 & 10.25 & F5 & 0.18 & 1.00 & 3.81 & 1.09 & 1.81 & 6.90 & - \\
\hline 113 & - & - & - & - & - & - & - & - & - & - & - & - & - \\
\hline 114 & 15.22 & 1.13 & 13.81 & 13.12 & 12.95 & - & - & 0.00 & - & - & - & - & 9.95 \\
\hline 115 & - & - & - & - & - & - & - & - & - & - & - & - & - \\
\hline 116 & 16.92 & 1.60 & 15.35 & 14.59 & 14.49 & M5 & 0.00 & 0.00 & 3.49 & -1.15 & 0.15 & 6.67 & 1.47 \\
\hline 117 & - & - & - & - & - & - & - & - & - & - & - & - & - \\
\hline 118 & 16.88 & 1.75 & 15.11 & 14.50 & 14.45 & - & - & 0.00 & - & - & - & - & 1.55 \\
\hline 119 & 15.67 & 1.65 & 13.68 & 12.96 & 12.59 & M3 & 1.25 & 0.00 & 3.53 & -0.48 & 0.32 & 6.25 & 7.64 \\
\hline 120 & 13.97 & 0.87 & 12.70 & 12.02 & 11.80 & K6 & 0.76 & 0.00 & 3.62 & -0.00 & 0.93 & 6.41 & - \\
\hline
\end{tabular}


Table 4. (Continued)

\begin{tabular}{|c|c|c|c|c|c|c|c|c|c|c|c|c|c|}
\hline$\overline{\mathrm{N}}$ & 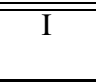 & "R-I & $\overline{\mathrm{J}}$ & $\overline{\mathrm{H}}$ & $\overline{\bar{K}}$ & $\overline{\text { Sp.T. }}$ & $\overline{\mathrm{Anv}}$ & $\overline{\mathrm{Av}(\mathrm{JHK})}$ & $\begin{array}{c}\log T_{\text {eff }} \\
{[\mathrm{K}]} \\
\end{array}$ & $\begin{array}{c}\log L_{b o l} \\
{\left[L_{\odot}\right]} \\
\end{array}$ & $\begin{array}{l}\text { Mass } \\
{\left[M_{\odot}\right]} \\
\end{array}$ & $\begin{array}{c}\text { LogAge } \\
\text { [yr.] }\end{array}$ & $\begin{array}{l}P_{r o t} \\
{[\mathrm{~d}]} \\
\end{array}$ \\
\hline 121 & 12.55 & 0.84 & 11.50 & 10.80 & 10.66 & - & - & $\overline{0.00}$ & - & - & - & - & - \\
\hline 122 & 17.60 & 1.87 & 15.68 & 15.13 & 14.67 & - & - & 0.00 & - & - & - & - & - \\
\hline 123 & 15.95 & 1.53 & 14.12 & 13.39 & 13.04 & M3.5 & 0.13 & 0.00 & 3.52 & -0.83 & 0.27 & 6.55 & 5.22 \\
\hline 124 & 15.56 & 1.26 & 13.92 & 13.22 & 12.95 & M2.5 & 0.00 & 0.57 & 3.54 & -0.75 & 0.34 & 6.58 & 0.51 \\
\hline 125 & 19.25 & 2.37 & 16.60 & 14.83 & 14.36 & - & - & - & - & - & - & - & - \\
\hline 126 & 16.35 & 1.85 & 14.46 & 13.87 & 13.56 & M5 & 0.00 & 0.00 & 3.49 & -0.92 & 0.18 & 6.52 & - \\
\hline 127 & 14.06 & 1.13 & 11.89 & 10.82 & 10.05 & K3 & 2.81 & 1.56 & 3.67 & 0.50 & 1.74 & 6.37 & - \\
\hline 128 & 17.31 & 1.35 & 15.89 & 15.29 & 14.78 & - & - & 0.00 & - & - & - & - & - \\
\hline 129 & 15.16 & 1.12 & 13.22 & 12.32 & 11.76 & M1 & 0.45 & 0.00 & 3.57 & -0.50 & 0.47 & 6.50 & 6.33 \\
\hline 130 & 15.85 & 0.94 & 14.67 & 14.00 & 13.71 & - & - & 0.00 & - & - & - & - & 0.46 \\
\hline 131 & 14.75 & 1.11 & 12.77 & 11.79 & 11.02 & K3 & 2.72 & 0.00 & 3.67 & 0.20 & 1.40 & 6.73 & - \\
\hline 132 & 15.39 & 1.80 & 13.62 & 12.94 & 12.72 & M5 & 0.00 & 0.00 & 3.49 & -0.54 & 0.22 & 6.19 & - \\
\hline 133 & 13.65 & 1.30 & 12.12 & 11.44 & 11.17 & - & - & 0.48 & - & - & - & - & - \\
\hline 134 & 16.75 & 2.05 & - & - & - & M5 & 0.67 & - & 3.49 & -0.92 & 0.18 & 6.52 & 1.21 \\
\hline 135 & 13.46 & 0.79 & 12.22 & 11.50 & 11.25 & K5 & 0.71 & 0.59 & 3.64 & 0.20 & 1.12 & 6.31 & 11.73 \\
\hline 136 & 16.49 & 1.91 & 13.80 & 12.50 & 11.98 & M3.5 & 1.83 & 5.72 & 3.52 & -0.63 & 0.29 & 6.36 & - \\
\hline 137 & 15.41 & 1.19 & 14.11 & 13.45 & 13.19 & - & - & 0.00 & - & - & - & - & 8.64 \\
\hline 138 & 17.00 & 1.50 & 15.50 & 15.02 & 14.56 & - & - & 0.00 & - & - & - & - & 3.92 \\
\hline 139 & 17.03 & 1.86 & 14.98 & 14.32 & 14.05 & M5 & 0.00 & 0.00 & 3.49 & -1.19 & 0.15 & 6.70 & 1.13 \\
\hline 140 & 16.11 & 1.63 & 14.48 & 13.88 & 13.64 & M5 & 0.00 & 0.00 & 3.49 & -0.83 & 0.19 & 6.27 & - \\
\hline 141 & - & - & - & 15.05 & 12.88 & - & - & - & - & - & - & - & - \\
\hline 142 & 14.43 & 1.05 & 12.95 & 12.11 & 11.82 & K7 & 1.29 & 1.54 & 3.61 & -0.06 & 0.77 & 6.32 & 3.65 \\
\hline 143 & 14.23 & 1.03 & 12.87 & 11.91 & 11.27 & M0 & 0.62 & 0.97 & 3.59 & -0.10 & 0.56 & 6.13 & - \\
\hline 144 & - & - & - & - & - & - & - & - & - & - & - & - & - \\
\hline 145 & - & - & - & - & - & - & - & - & - & - & - & - & - \\
\hline 146 & 15.21 & 2.01 & 13.31 & 12.48 & 12.08 & - & - & 2.05 & - & - & - & - & - \\
\hline 147 & - & - & 14.84 & 14.07 & 13.71 & - & - & 0.00 & - & - & - & - & - \\
\hline 148 & 15.43 & 1.49 & 13.62 & 12.91 & 12.68 & $>\mathrm{M} 4$ & - & 0.00 & - & - & - & - & - \\
\hline 149 & - & - & 10.22 & 10.23 & 10.17 & - & - & 0.00 & - & - & - & - & - \\
\hline 150 & - & - & - & 16.48 & 14.78 & - & - & - & - & - & - & - & - \\
\hline 151 & 14.29 & 1.68 & 12.41 & 11.58 & 11.13 & M4 & 0.22 & 0.00 & 3.51 & -0.12 & 0.27 & 5.60 & - \\
\hline 152 & - & - & - & - & - & - & - & - & - & - & - & - & - \\
\hline 153 & 16.30 & 1.26 & 14.88 & 14.31 & 13.99 & - & - & 0.00 & - & - & - & - & - \\
\hline 154 & 12.49 & 0.61 & 11.72 & 10.95 & 10.51 & K2.5 & 0.56 & 0.00 & 3.68 & 0.57 & 1.90 & 6.38 & - \\
\hline 155 & 14.95 & 1.06 & 13.66 & 12.98 & 12.72 & M0 & 0.76 & 0.43 & 3.59 & -0.35 & 0.58 & 6.47 & 6.98 \\
\hline 156 & - & - & - & - & 14.05 & - & - & - & - & - & - & - & - \\
\hline 157 & 14.43 & - & 13.00 & 12.30 & 12.05 & M1 & - & 0.47 & 3.57 & - & - & - & - \\
\hline 158 & 15.26 & - & - & - & - & - & - & - & - & - & - & - & - \\
\hline 159 & 17.08 & - & 14.69 & 13.59 & 13.10 & - & - & 4.21 & - & - & - & - & - \\
\hline 160 & 15.49 & - & - & - & - & - & - & - & - & - & - & - & - \\
\hline 161 & 16.50 & - & 11.78 & 9.32 & 7.77 & - & - & 14.79 & - & - & - & - & - \\
\hline 162 & - & - & - & - & - & - & - & - & - & - & - & - & - \\
\hline 163 & - & - & 12.91 & 11.87 & 11.19 & - & - & 1.88 & - & - & - & - & - \\
\hline 164 & 14.69 & 1.26 & 13.26 & 12.45 & 12.09 & M3 & 0.00 & 1.77 & 3.53 & -0.39 & 0.32 & 6.18 & - \\
\hline 165 & - & - & - & - & 12.86 & - & - & - & - & - & - & - & - \\
\hline 166 & 16.52 & - & 13.69 & 12.79 & 12.27 & - & - & 1.17 & - & - & - & - & - \\
\hline 167 & 13.34 & 1.12 & 11.59 & 10.70 & 10.31 & K6 & 1.87 & 2.40 & 3.62 & 0.52 & 0.92 & 5.77 & 3.35 \\
\hline 168 & - & - & - & - & - & - & - & - & - & - & - & - & - \\
\hline 169 & 17.53 & 2.31 & 14.90 & 14.11 & 13.63 & M6 & 0.04 & 0.00 & 3.48 & -1.32 & - & - & 5.43 \\
\hline 170 & 14.34 & 0.90 & 13.19 & 12.41 & 12.08 & - & - & 1.35 & - & - & - & - & 6.84 \\
\hline 171 & 19.60 & 2.07 & - & - & 13.43 & - & - & - & - & - & - & - & - \\
\hline 172 & 14.21 & 0.83 & 13.09 & 12.41 & 12.20 & K7 & 0.31 & 0.00 & 3.61 & -0.22 & 0.78 & 6.56 & 4.17 \\
\hline 173 & 20.32 & - & 16.33 & 14.27 & 13.15 & - & - & 13.54 & - & - & - & - & - \\
\hline 174 & - & - & - & 13.44 & 11.43 & - & - & - & - & - & - & - & - \\
\hline 175 & 16.75 & 1.63 & 14.93 & 14.21 & 13.89 & - & - & 0.00 & - & - & - & - & - \\
\hline 176 & 13.20 & 0.68 & 12.44 & 11.65 & 11.22 & K4 & 0.80 & 0.00 & 3.66 & 0.34 & 1.50 & 6.42 & 5.22 \\
\hline 177 & 17.27 & 2.25 & 14.22 & 12.65 & 11.85 & - & - & 8.80 & - & - & - & - & 6.61 \\
\hline 178 & 17.78 & 2.24 & - & - & - & - & - & - & - & - & - & - & 2.82 \\
\hline 179 & 11.87 & 0.81 & 10.58 & 9.71 & 9.21 & K1: & - & 0.84 & - & - & - & - & - \\
\hline 180 & - & - & - & - & - & - & - & - & - & - & - & - & - \\
\hline
\end{tabular}


Table 4. (Continued)

\begin{tabular}{|c|c|c|c|c|c|c|c|c|c|c|c|c|c|}
\hline$\overline{\mathrm{N}}$ & $\overline{\mathrm{II}}$ & $\overline{\overline{R-I}}$ & $\overline{\mathrm{J}}$ & $\overline{\overline{\mathrm{H}}}$ & $\overline{\overline{\mathrm{K}}}$ & $\overline{\text { Sp.T. }}$ & 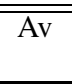 & 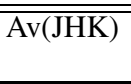 & $\begin{array}{c}\log T_{e f f} \\
{[\mathrm{~K}]}\end{array}$ & $\begin{array}{c}\log L_{b o l} \\
{\left[L_{\odot}\right]}\end{array}$ & $\begin{array}{l}\text { Mass } \\
{\left[M_{\odot}\right]}\end{array}$ & $\begin{array}{c}\text { LogAge } \\
\text { [yr.] }\end{array}$ & $\begin{array}{l}P_{\text {rot }} \\
{[\mathrm{d}]}\end{array}$ \\
\hline$\overline{181}$ & 14.71 & $\overline{-}$ & - & - & $\overline{-1}$ & - & $\overline{-}$ & $\overline{-}$ & $\overline{-}$ & $\overline{-}$ & $\overline{-}$ & $\overline{-}$ & $\overline{-1}$ \\
\hline 182 & - & - & - & 14.53 & 13.01 & - & - & - & - & - & - & - & - \\
\hline 183 & 15.25 & 1.03 & - & - & - & - & - & - & - & - & - & - & - \\
\hline 184 & 13.36 & 0.69 & 12.35 & 11.71 & 11.55 & K5 & 0.27 & 0.00 & 3.64 & 0.13 & 1.12 & 6.41 & 0.91 \\
\hline 185 & 10.56 & 0.11 & 10.45 & 10.40 & 10.36 & A0 & 0.49 & 0.00 & 3.98 & 1.68 & 2.50 & 6.64 & - \\
\hline 186 & - & - & - & - & - & - & - & - & - & - & - & - & - \\
\hline 187 & 13.30 & 0.71 & 12.24 & 11.61 & 11.38 & K4 & 0.94 & 0.00 & 3.66 & 0.33 & 1.49 & 6.42 & - \\
\hline 188 & 17.20 & 2.30 & - & - & 13.63 & - & - & - & - & - & - & - & - \\
\hline 189 & 14.15 & 0.73 & - & - & - & - & - & - & - & - & - & - & 5.15 \\
\hline 190 & 13.30 & 0.60 & 12.36 & 11.82 & 11.65 & K0 & 0.94 & 1.86 & 3.72 & 0.36 & 1.47 & 6.99 & - \\
\hline 191 & 16.42 & 1.85 & 14.34 & 13.53 & 13.14 & - & - & 0.00 & - & - & - & - & - \\
\hline 192 & 18.10 & 2.77 & - & - & - & - & - & - & - & - & - & - & - \\
\hline 193 & 13.90 & 0.71 & 12.85 & 12.23 & 12.05 & K5 & 0.36 & 0.00 & 3.64 & -0.07 & 1.09 & 6.70 & 4.26 \\
\hline 194 & 19.89 & - & - & - & - & - & - & - & - & - & - & - & - \\
\hline 195 & - & - & - & 15.07 & 13.66 & - & - & - & - & - & - & - & - \\
\hline 196 & - & - & 9.28 & 9.27 & 9.28 & - & - & 0.00 & - & - & - & - & - \\
\hline 197 & 15.77 & 1.70 & 13.28 & 11.89 & 11.16 & M1 & 3.03 & 7.33 & 3.57 & -0.11 & 0.48 & 6.08 & - \\
\hline 198 & - & - & - & - & - & - & - & - & - & - & - & - & - \\
\hline 199 & - & - & 16.88 & 14.14 & 12.63 & - & - & 19.84 & - & - & - & - & - \\
\hline 200 & 15.53 & 1.18 & - & - & 13.31 & M0 & 1.29 & - & 3.59 & -0.45 & 0.59 & 6.62 & 3.63 \\
\hline 201 & 16.99 & 2.34 & 14.44 & 13.76 & 13.36 & - & - & 0.00 & - & - & - & - & 3.11 \\
\hline 202 & 15.75 & 1.68 & - & 12.26 & - & M3 & 1.38 & - & 3.53 & -0.48 & 0.32 & 6.25 & - \\
\hline 203 & 14.17 & 0.87 & 12.93 & 12.24 & 12.07 & M1 & 0.00 & 0.00 & 3.57 & -0.22 & 0.47 & 6.16 & 0.91 \\
\hline 204 & 14.50 & 1.37 & 12.97 & 12.27 & 12.02 & M2: & - & 0.00 & - & - & - & - & 1.31 \\
\hline 205 & 16.76 & 2.11 & 14.54 & 13.75 & 13.38 & - & - & 0.00 & - & - & - & - & 1.13 \\
\hline 206 & 11.95 & 0.42 & 11.39 & 11.04 & 10.95 & G3 & 0.27 & 0.00 & 3.77 & 0.73 & 1.57 & 7.01 & 2.16 \\
\hline 207 & 14.68 & 1.75 & 12.81 & 12.03 & 11.69 & - & - & 1.43 & - & - & - & - & 9.71 \\
\hline 208 & 16.80 & 1.74 & - & - & - & - & - & - & - & - & - & - & - \\
\hline 209 & - & - & - & 14.79 & 13.30 & - & - & - & - & - & - & - & - \\
\hline 210 & 15.24 & 1.30 & 13.86 & 13.03 & 12.76 & M1 & 1.25 & 1.41 & 3.57 & -0.34 & 0.47 & 6.29 & - \\
\hline 211 & 16.22 & 1.23 & - & - & - & - & - & - & - & - & - & - & - \\
\hline 212 & 14.45 & 0.84 & 13.34 & 12.67 & 12.49 & K4 & 1.52 & 0.00 & 3.66 & 0.01 & 1.28 & 6.86 & 4.35 \\
\hline 213 & 17.18 & 1.97 & 14.00 & 12.37 & 11.65 & - & - & 8.92 & - & - & - & - & 8.84 \\
\hline 214 & 16.21 & 1.79 & 14.52 & 13.87 & 13.58 & - & - & 0.00 & - & - & - & - & - \\
\hline 215 & 18.74 & 2.67 & 15.20 & 13.81 & - & - & - & - & - & - & - & - & 4.95 \\
\hline 216 & 14.41 & 1.17 & 13.02 & 12.34 & 12.10 & M2.5 & 0.00 & 0.00 & 3.54 & -0.29 & 0.36 & 6.13 & 7.49 \\
\hline 217 & 17.29 & 2.37 & 14.78 & 14.20 & 13.70 & - & - & 0.00 & - & - & - & - & 1.12 \\
\hline 218 & 17.33 & 1.81 & 15.60 & 14.93 & 14.55 & - & - & 0.00 & - & - & - & - & 1.71 \\
\hline 219 & 16.43 & 1.72 & 14.67 & 13.98 & 13.84 & - & - & 0.00 & - & - & - & - & - \\
\hline 220 & 18.77 & 2.27 & 16.19 & 15.02 & 14.40 & M & - & 5.30 & - & - & - & - & 1.66 \\
\hline 221 & 15.87 & 1.56 & 14.25 & 13.60 & 13.30 & M3 & 0.85 & 0.00 & 3.53 & -0.65 & 0.32 & 6.41 & 1.30 \\
\hline 222 & - & - & - & - & - & - & - & - & - & - & - & - & - \\
\hline 223 & 15.84 & 1.40 & 14.41 & 13.71 & 13.49 & - & - & 0.00 & - & - & - & - & 1.11 \\
\hline 224 & 17.00 & 0.95 & - & - & - & - & - & - & - & - & - & - & - \\
\hline 225 & 15.58 & 1.24 & 13.93 & 13.11 & 12.64 & - & - & 0.00 & - & - & - & - & - \\
\hline 226 & 15.48 & 1.45 & 13.97 & 13.21 & 13.03 & M & - & 0.00 & - & - & - & - & 0.90 \\
\hline 227 & 16.05 & 1.42 & 14.62 & 13.91 & 13.71 & M3 & 0.22 & 0.00 & 3.53 & -0.88 & 0.30 & 6.65 & 5.66 \\
\hline 228 & - & - & - & - & - & - & - & - & - & - & - & - & - \\
\hline 229 & 10.89 & 0.09 & 10.64 & 10.51 & 10.44 & A0 & 0.40 & 0.00 & 3.98 & 1.52 & 2.46 & 6.75 & - \\
\hline 230 & - & - & - & - & - & - & - & - & - & - & - & - & - \\
\hline 231 & 15.60 & 1.27 & 14.13 & 13.37 & 13.16 & M2 & 0.49 & 0.72 & 3.55 & -0.66 & 0.38 & 6.53 & 7.49 \\
\hline 232 & - & - & - & - & - & - & - & - & - & - & - & - & - \\
\hline 233 & - & - & - & - & - & - & - & - & - & - & - & - & - \\
\hline 234 & 13.72 & 0.88 & 12.48 & 11.85 & 11.64 & K5 & 1.12 & 0.00 & 3.64 & 0.19 & 1.11 & 6.31 & - \\
\hline 235 & - & - & - & - & - & - & - & - & - & - & - & - & - \\
\hline 236 & - & - & - & - & - & - & - & - & - & - & - & - & - \\
\hline 237 & - & - & - & - & - & - & - & - & - & - & - & - & - \\
\hline 238 & 15.19 & 1.53 & 13.13 & 12.36 & 12.07 & M3 & 0.71 & 1.07 & 3.53 & -0.42 & 0.32 & 6.20 & 1.90 \\
\hline 239 & - & - & - & - & 14.12 & - & - & - & - & - & - & - & - \\
\hline 240 & 13.55 & 0.96 & 12.37 & 11.59 & 11.30 & K5 & 1.47 & 1.13 & 3.64 & 0.35 & 1.12 & 6.11 & 12.88 \\
\hline
\end{tabular}


Table 4. (Continued)

\begin{tabular}{|c|c|c|c|c|c|c|c|c|c|c|c|c|c|}
\hline$\overline{\mathrm{N}}$ & $\overline{\bar{I}}$ & $\overline{\bar{R}-I}$ & $\overline{\mathrm{J}}$ & $\overline{\overline{\mathrm{H}}}$ & $\overline{\bar{K}}$ & $\overline{\text { Sp.T. }}$ & $\overline{\overline{\mathrm{Av}}}$ & $\overline{\mathrm{Av}(\mathrm{JHK})}$ & $\begin{array}{c}\log T_{e f f} \\
{[\mathrm{~K}]}\end{array}$ & $\begin{array}{c}\log L_{b o l} \\
{\left[L_{\odot}\right]}\end{array}$ & $\begin{array}{l}\text { Mass } \\
{\left[M_{\odot}\right]}\end{array}$ & $\begin{array}{c}\text { LogAge } \\
\text { [yr.] }\end{array}$ & $\begin{array}{l}P_{r o t} \\
{[\mathrm{~d}]}\end{array}$ \\
\hline 241 & $\bar{~} 16.51$ & 1.58 & 14.86 & 14.18 & - & $\overline{-}$ & $\overline{-1}$ & $\overline{-1}$ & $\overline{-1}$ & $\overline{-1}$ & - & - & 4.35 \\
\hline 242 & - & - & 16.26 & 12.73 & 10.56 & - & - & 24.90 & - & - & - & - & - \\
\hline 243 & 17.25 & 2.20 & - & - & - & - & - & - & - & - & - & - & 1.50 \\
\hline 244 & - & - & - & - & - & - & - & - & - & - & - & _- & - \\
\hline 245 & 13.85 & 1.50 & 12.27 & 11.54 & 11.31 & M3 & 0.58 & 0.56 & 3.53 & 0.09 & 0.32 & 5.17 & - \\
\hline 246 & 16.22 & 1.43 & - & 13.42 & 13.06 & - & - & - & - & - & - & - & 11.73 \\
\hline 247 & 13.01 & 0.44 & 12.37 & 11.90 & 11.79 & - & - & 0.00 & - & - & - & - & - \\
\hline 248 & 20.41 & -1.09 & - & - & - & - & - & - & - & - & - & - & - \\
\hline 249 & 12.52 & 0.48 & 11.64 & 11.02 & 10.55 & G5 & 0.49 & 0.00 & 3.76 & 0.55 & 1.40 & 7.14 & 3.00 \\
\hline 250 & 16.16 & 1.86 & 14.11 & 13.35 & 13.05 & M5 & 0.00 & 1.10 & 3.49 & -0.85 & 0.19 & 6.42 & 2.55 \\
\hline 251 & 18.56 & 3.06 & 14.78 & 13.27 & 12.43 & - & - & 8.40 & - & - & - & - & - \\
\hline 252 & 15.10 & 1.67 & 12.93 & 12.09 & 11.68 & M3 & 1.34 & 0.00 & 3.53 & -0.23 & 0.32 & 6.07 & 3.16 \\
\hline 253 & - & - & - & - & 14.91 & - & - & - & - & - & - & - & - \\
\hline 254 & 15.15 & 1.12 & 13.85 & 13.12 & 12.98 & M1.5 & 0.13 & 0.00 & 3.56 & -0.57 & 0.42 & 6.49 & - \\
\hline 255 & 17.73 & 2.48 & 14.12 & 12.36 & 11.53 & - & - & 10.15 & - & - & - & - & - \\
\hline 256 & - & - & - & - & 15.04 & - & - & - & - & - & - & - & - \\
\hline 257 & - & - & - & - & 14.33 & - & - & - & - & - & - & _- & - \\
\hline 258 & 13.39 & 0.37 & 12.81 & 12.50 & 12.44 & G5 & 0.00 & 0.00 & 3.76 & 0.09 & 1.14 & 7.82 & - \\
\hline 259 & - & - & - & - & - & - & - & - & - & - & - & - & - \\
\hline 260 & 13.59 & 1.22 & 11.76 & 10.28 & 9.23 & G6: & - & 5.05 & - & - & - & - & - \\
\hline 261 & 11.64 & 0.72 & 10.43 & 9.53 & 8.86 & G & - & 0.00 & - & - & - & - & - \\
\hline 262 & 15.33 & 1.62 & 13.57 & 12.84 & 12.59 & - & - & 0.65 & - & - & - & - & 1.07 \\
\hline 263 & 15.47 & 1.18 & 13.41 & 12.55 & 11.93 & M0 & 1.29 & 0.00 & 3.59 & -0.43 & 0.59 & 6.59 & 3.14 \\
\hline 264 & - & - & - & - & - & - & - & - & - & - & - & - & - \\
\hline 265 & 13.33 & 0.89 & 12.20 & 11.54 & 11.36 & M0 & 0.00 & 0.00 & 3.59 & 0.11 & 0.56 & 5.92 & 9.48 \\
\hline 266 & 16.93 & 1.65 & 15.30 & 14.65 & 14.54 & - & - & 0.00 & - & - & - & - & 1.73 \\
\hline 267 & 19.57 & 2.22 & - & - & - & - & - & - & - & - & - & - & - \\
\hline 268 & 15.36 & 1.32 & 13.96 & 13.39 & 13.13 & - & - & 0.00 & - & - & - & - & - \\
\hline 269 & 13.71 & 0.84 & 12.59 & 11.95 & 11.68 & K4 & 1.52 & 0.00 & 3.66 & 0.31 & 1.50 & 6.46 & 0.88 \\
\hline 270 & - & - & - & 14.30 & 12.10 & - & - & - & - & - & - & - & - \\
\hline 271 & 15.46 & 1.36 & 14.03 & 13.42 & 13.21 & M2.5 & 0.42 & 0.00 & 3.54 & -0.61 & 0.35 & 6.42 & - \\
\hline 272 & 16.88 & 1.65 & 15.15 & 14.47 & - & M4 & 0.09 & - & 3.51 & -1.19 & 0.21 & 6.81 & 1.81 \\
\hline 273 & 18.08 & 2.50 & 14.18 & 12.39 & 11.16 & - & - & 7.83 & - & - & - & - & - \\
\hline 274 & - & - & - & - & - & - & - & - & - & - & - & - & - \\
\hline 275 & 14.04 & 0.94 & 12.81 & 12.04 & 11.85 & M0 & 0.22 & 0.00 & 3.59 & -0.12 & 0.57 & 6.17 & 1.97 \\
\hline 276 & 14.35 & 1.12 & 13.11 & 12.34 & 12.18 & M1.5 & 0.13 & 0.00 & 3.56 & -0.25 & 0.43 & 6.14 & 2.67 \\
\hline 277 & 16.30 & 1.59 & 13.98 & 12.69 & 12.12 & - & - & 5.77 & - & - & - & - & - \\
\hline 278 & 14.23 & 0.70 & 13.26 & 12.64 & 12.48 & K4 & 0.89 & 0.00 & 3.66 & -0.05 & 1.20 & 6.94 & 3.88 \\
\hline 279 & - & - & - & - & - & - & - & - & - & - & - & - & - \\
\hline 280 & 16.91 & 2.06 & 13.60 & 12.09 & 11.31 & - & - & 8.33 & - & - & - & - & 6.01 \\
\hline 281 & 15.16 & 1.69 & 12.97 & 11.90 & 11.50 & - & - & 3.64 & - & - & - & - & - \\
\hline 282 & 16.56 & 1.93 & 13.63 & 12.31 & 11.55 & - & - & 5.18 & - & - & - & - & - \\
\hline 283 & - & - & - & - & - & - & - & - & - & - & - & - & - \\
\hline 284 & 16.10 & 1.48 & 14.54 & 13.86 & 13.65 & - & - & 0.00 & - & - & - & - & - \\
\hline 285 & 16.70 & 2.03 & 14.62 & 13.98 & 13.69 & - & - & 0.00 & - & - & - & - & 1.32 \\
\hline 286 & - & - & - & - & - & - & - & - & - & - & - & - & - \\
\hline 287 & - & - & - & - & 11.82 & - & - & - & - & - & - & - & - \\
\hline 288 & 17.20 & 1.97 & 15.44 & 14.40 & - & M6 & 0.00 & - & 3.48 & -1.20 & 0.11 & 6.61 & 1.59 \\
\hline 289 & - & - & - & - & - & - & - & - & - & - & - & - & - \\
\hline 290 & 13.85 & 0.63 & 12.90 & 12.28 & 12.17 & K5 & 0.00 & 0.00 & 3.64 & -0.13 & 1.11 & 6.82 & 3.92 \\
\hline 291 & 14.72 & 1.57 & 12.96 & 12.14 & - & M3 & 0.89 & - & 3.53 & -0.18 & 0.33 & 6.04 & 0.80 \\
\hline 292 & 17.07 & 2.49 & 13.72 & 12.25 & 11.23 & - & - & 4.97 & - & - & - & - & - \\
\hline 293 & 14.44 & 1.05 & 12.94 & 11.96 & 11.54 & K7 & 1.29 & 3.12 & 3.61 & -0.07 & 0.77 & 6.33 & 5.83 \\
\hline 294 & 13.83 & 1.11 & 12.44 & 11.67 & 11.52 & M2 & 0.00 & 0.00 & 3.55 & -0.07 & 0.39 & 5.92 & 0.86 \\
\hline 295 & - & - & - & - & - & - & - & - & - & - & - & - & - \\
\hline 296 & - & - & 15.10 & - & - & - & - & - & - & - & - & - & - \\
\hline 297 & - & - & 7.66 & 7.81 & 7.87 & - & - & 0.00 & - & - & - & - & - \\
\hline 298 & 19.66 & - & 15.62 & 13.72 & 12.88 & - & - & 11.63 & - & - & - & - & - \\
\hline 299 & - & - & - & - & - & - & - & - & - & - & - & - & - \\
\hline 300 & 12.78 & 0.89 & 11.54 & 10.88 & 10.64 & K5 & 1.16 & 0.00 & 3.64 & 0.58 & 1.15 & 5.85 & 2.93 \\
\hline
\end{tabular}


Table 4. (Continued)

\begin{tabular}{|c|c|c|c|c|c|c|c|c|c|c|c|c|c|}
\hline$\overline{\mathrm{N}}$ & 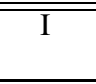 & R-I & $\overline{\bar{J}}$ & $\overline{\overline{\mathrm{H}}}$ & $\overline{\overline{\mathrm{K}}}$ & $\overline{\text { Sp.T. }}$ & 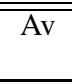 & 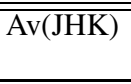 & $\begin{array}{c}\log T_{e f f} \\
{[\mathrm{~K}]}\end{array}$ & $\begin{array}{c}\log L_{b o l} \\
{\left[L_{\odot}\right]} \\
\end{array}$ & $\begin{array}{l}\text { Mass } \\
{\left[M_{\odot}\right]} \\
\end{array}$ & $\begin{array}{c}\begin{array}{c}\text { LogAge } \\
\text { [yr.] }\end{array} \\
\end{array}$ & $\begin{array}{l}P_{\text {rot }} \\
{[\mathrm{d}]}\end{array}$ \\
\hline 301 & 15.36 & 1.16 & 13.98 & 13.30 & - & - & - & - & - & - & - & - & - \\
\hline 302 & - & - & 9.61 & 9.40 & 9.32 & - & - & 0.00 & - & - & - & - & - \\
\hline 303 & - & - & - & - & - & - & - & - & - & - & - & - & - \\
\hline 304 & 15.12 & 1.52 & 13.48 & 12.79 & 12.61 & M3.5 & 0.09 & 0.00 & 3.52 & -0.51 & 0.29 & 6.25 & - \\
\hline 305 & 17.28 & 0.98 & - & 7.64 & 4.92 & - & - & - & - & - & - & - & - \\
\hline 306 & 15.83 & 1.89 & - & - & - & - & - & - & - & - & - & - & 5.22 \\
\hline 307 & 15.07 & 1.38 & 13.52 & 12.80 & 12.56 & M3 & 0.04 & 0.49 & 3.53 & -0.53 & 0.32 & 6.29 & 0.68 \\
\hline 308 & - & - & - & - & - & - & - & - & - & - & - & - & - \\
\hline 309 & 16.45 & 0.76 & - & - & - & K5 & 0.58 & - & 3.64 & -1.03 & - & - & - \\
\hline 310 & - & - & - & 14.23 & - & - & - & - & - & - & - & - & - \\
\hline 311 & 15.15 & 1.25 & 13.36 & 12.23 & 11.50 & K7 & 2.19 & 2.56 & 3.61 & -0.13 & 0.80 & 6.44 & 9.04 \\
\hline 312 & 17.25 & 1.90 & 15.34 & 14.66 & 14.16 & M4 & 1.20 & 0.00 & 3.51 & -1.06 & 0.22 & 6.70 & - \\
\hline 313 & - & - & - & - & - & - & - & - & - & - & - & - & - \\
\hline 314 & - & - & - & - & - & - & - & - & - & - & - & - & - \\
\hline 315 & - & - & - & - & - & - & - & - & - & - & - & - & - \\
\hline 316 & 18.47 & 2.55 & 15.54 & 14.84 & 14.54 & - & - & 0.00 & - & - & - & - & 3.60 \\
\hline 317 & - & - & - & 15.15 & 12.22 & - & - & - & - & - & - & - & - \\
\hline 318 & 13.43 & 0.93 & 12.21 & 11.52 & 11.26 & K7 & 0.76 & 0.48 & 3.61 & 0.20 & 0.74 & 6.04 & - \\
\hline 319 & 16.91 & 1.68 & 14.66 & 13.36 & 12.57 & - & - & 4.70 & - & - & - & - & - \\
\hline 320 & 15.29 & 1.55 & - & - & - & - & - & - & - & - & - & - & 2.25 \\
\hline 321 & - & - & - & - & - & - & - & - & - & - & - & - & - \\
\hline 322 & 16.47 & 1.74 & 14.74 & 14.10 & 13.70 & - & - & 0.00 & - & - & - & - & 1.61 \\
\hline 323 & 15.88 & 1.78 & 14.11 & 13.56 & 13.25 & - & - & 0.00 & - & - & - & - & 1.05 \\
\hline 324 & - & - & - & 14.79 & - & - & - & - & - & - & - & - & - \\
\hline 325 & - & - & 9.37 & 9.40 & 9.38 & - & - & 0.00 & - & - & - & - & - \\
\hline 326 & 14.62 & 1.16 & - & 12.43 & 12.28 & - & - & - & - & - & - & - & 1.43 \\
\hline 327 & - & - & - & - & - & - & - & - & - & - & - & - & - \\
\hline 328 & 14.65 & 1.26 & - & - & - & - & - & - & - & - & - & - & 2.09 \\
\hline 329 & 10.71 & 0.34 & 10.30 & 9.98 & 9.90 & - & - & 0.00 & - & - & - & - & - \\
\hline 330 & 16.21 & 1.76 & 14.39 & 13.67 & 13.39 & M6 & 0.00 & 0.72 & 3.48 & -0.80 & 0.14 & 5.70 & 1.09 \\
\hline 331 & 16.57 & 1.71 & - & - & - & M4 & 0.36 & - & 3.51 & -1.00 & 0.23 & 6.65 & - \\
\hline 332 & 12.86 & 0.81 & 11.61 & 10.81 & 10.34 & K5 & 0.80 & 0.00 & 3.64 & 0.46 & 1.13 & 5.98 & 1.76 \\
\hline 333 & 14.64 & 1.69 & 12.74 & 12.03 & 11.68 & M4 & 0.27 & 0.00 & 3.51 & -0.25 & 0.27 & 5.67 & 4.17 \\
\hline 334 & 14.59 & 0.94 & 13.42 & 12.73 & 12.55 & K5 & 1.38 & 0.00 & 3.64 & -0.09 & 1.11 & 6.74 & 5.75 \\
\hline 335 & 15.76 & 1.69 & 13.48 & 12.31 & 11.77 & - & - & 4.90 & - & - & - & - & 2.38 \\
\hline 336 & - & - & - & - & - & - & - & - & - & - & - & - & - \\
\hline 337 & - & - & - & - & - & - & - & - & - & - & - & - & - \\
\hline 338 & 14.50 & 1.02 & 13.22 & 12.52 & 12.33 & M1 & 0.00 & 0.00 & 3.57 & -0.35 & 0.47 & 6.31 & 1.44 \\
\hline 339 & 14.58 & 1.58 & 13.03 & 12.33 & 12.11 & M3.5 & 0.36 & 0.00 & 3.52 & -0.23 & 0.29 & 5.98 & 3.06 \\
\hline 340 & 13.74 & 0.82 & 12.60 & 11.94 & 11.72 & K5 & 0.85 & 0.00 & 3.64 & 0.12 & 1.12 & 6.42 & 11.07 \\
\hline 341 & 15.19 & 1.06 & 13.88 & 13.19 & 12.95 & - & - & 0.00 & - & - & - & - & 0.84 \\
\hline 342 & 15.30 & 1.14 & 13.87 & 12.70 & 11.80 & K8.5 & 1.26 & 1.92 & 3.59 & -0.38 & 0.64 & 6.59 & - \\
\hline 343 & 14.26 & 1.49 & 12.52 & 11.69 & 11.43 & M3 & 0.54 & 1.35 & 3.53 & -0.09 & 0.32 & 5.79 & 1.37 \\
\hline 344 & 14.44 & 1.12 & 13.00 & 12.06 & 11.60 & M0.5 & 0.74 & 3.08 & 3.58 & -0.15 & 0.53 & 6.16 & 8.28 \\
\hline 345 & 15.02 & 1.05 & 13.72 & 12.99 & 12.70 & M1 & 0.13 & 0.81 & 3.57 & -0.53 & 0.47 & 6.52 & - \\
\hline 346 & 16.30 & 1.44 & 14.96 & 14.13 & 13.73 & - & - & 0.00 & - & - & - & - & - \\
\hline 347 & - & - & 9.39 & 9.28 & 9.23 & A7 & - & 0.00 & 3.89 & - & - & - & - \\
\hline 348 & 14.43 & 1.24 & 12.71 & 11.93 & 11.61 & M3.5 & 0.00 & 1.32 & 3.52 & -0.26 & 0.29 & 6.03 & 0.61 \\
\hline 349 & 12.39 & 0.33 & 11.88 & 11.61 & 11.56 & - & - & 0.00 & - & - & - & - & - \\
\hline 350 & 12.31 & 0.79 & 11.31 & 10.67 & 10.44 & K5 & 0.71 & 0.00 & 3.64 & 0.66 & 1.16 & 5.77 & 6.30 \\
\hline 351 & 19.82 & - & 16.26 & 14.57 & 13.79 & - & - & 9.46 & - & - & - & - & - \\
\hline 352 & 13.34 & 1.06 & 11.27 & 10.50 & 10.07 & K5 & 1.92 & 0.00 & 3.64 & 0.54 & 1.14 & 5.89 & - \\
\hline 353 & 13.64 & 0.69 & 12.65 & 11.88 & 11.50 & K4 & 0.85 & 0.00 & 3.66 & 0.17 & 1.40 & 6.64 & 5.20 \\
\hline 354 & 15.07 & 1.45 & 13.53 & 12.86 & 12.62 & M3.5 & 0.00 & 0.00 & 3.52 & -0.51 & 0.29 & 6.26 & 0.44 \\
\hline 355 & 15.82 & 1.32 & 14.27 & 13.59 & 13.36 & M2.5 & 0.25 & 0.00 & 3.54 & -0.79 & 0.34 & 6.63 & - \\
\hline 356 & 16.60 & 1.87 & 13.74 & 11.99 & 11.36 & - & - & 10.13 & - & - & - & - & 2.71 \\
\hline 357 & 16.88 & 1.62 & 14.36 & 12.75 & 11.87 & - & - & 9.28 & - & - & - & - & 0.97 \\
\hline 358 & 19.17 & 2.55 & 16.08 & 15.65 & 15.27 & - & - & 0.00 & - & - & - & - & 1.38 \\
\hline 359 & 13.53 & 1.18 & 12.12 & 11.35 & 11.14 & M3 & 0.00 & 0.95 & 3.53 & 0.07 & 0.32 & 5.20 & 2.43 \\
\hline 360 & 13.77 & 0.87 & 12.63 & 11.97 & 11.78 & M0 & 0.00 & 0.00 & 3.59 & -0.07 & 0.56 & 6.10 & 6.51 \\
\hline
\end{tabular}


Table 4. (Continued)

\begin{tabular}{|c|c|c|c|c|c|c|c|c|c|c|c|c|c|}
\hline$\overline{\mathrm{N}}$ & 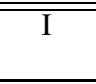 & "R-I & $\overline{\mathrm{J}}$ & $\overline{\mathrm{H}}$ & $\overline{\bar{K}}$ & $\overline{\text { Sp.T. }}$ & $\overline{\overline{\mathrm{Av}}}$ & 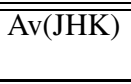 & $\begin{array}{c}\log T_{e f f} \\
{[\mathrm{~K}]} \\
\end{array}$ & $\begin{array}{c}\log L_{b o l} \\
{\left[L_{\odot}\right]} \\
\end{array}$ & $\begin{array}{l}\text { Mass } \\
{\left[M_{\odot}\right]} \\
\end{array}$ & $\begin{array}{c}\text { LogAge } \\
\text { [yr.] }\end{array}$ & $\begin{array}{l}P_{r o t} \\
{[\mathrm{~d}]}\end{array}$ \\
\hline 361 & 15.43 & 1.62 & 13.33 & 12.30 & 11.85 & $\overline{4}$ M2.5 & 1.58 & 3.58 & 3.54 & $\begin{array}{c}-0.31 \\
\end{array}$ & 0.36 & 6.14 & 2.91 \\
\hline 362 & 15.65 & 1.70 & 13.92 & 13.31 & 13.07 & M3 & 1.47 & 0.00 & 3.53 & -0.41 & 0.32 & 6.20 & 0.96 \\
\hline 363 & - & - & - & 16.31 & 13.15 & - & - & - & - & - & - & - & - \\
\hline 364 & 13.57 & 0.68 & 12.49 & 11.84 & 11.61 & K4 & 0.80 & 0.00 & 3.66 & 0.19 & 1.40 & 6.61 & - \\
\hline 365 & 15.75 & 1.35 & 14.33 & 13.63 & 13.38 & M0 & 2.05 & 0.00 & 3.59 & -0.35 & 0.58 & 6.47 & 1.45 \\
\hline 366 & - & - & - & - & - & - & - & - & - & - & - & - & - \\
\hline 367 & 14.69 & 1.01 & 13.41 & 12.65 & 12.46 & M1 & 0.00 & 0.00 & 3.57 & -0.43 & 0.47 & 6.40 & 11.32 \\
\hline 368 & 15.79 & 1.48 & 14.31 & 13.74 & 13.45 & - & - & 0.00 & - & - & - & - & 0.71 \\
\hline 369 & 16.65 & 1.65 & 15.04 & 14.41 & 14.03 & - & - & 0.00 & - & - & - & - & 5.15 \\
\hline 370 & 14.75 & 1.63 & 13.12 & 12.47 & 12.19 & M5 & 0.00 & 0.00 & 3.49 & -0.28 & 0.23 & 5.28 & - \\
\hline 371 & 16.20 & 1.47 & 14.54 & 13.76 & 13.51 & - & - & 0.00 & - & - & - & - & - \\
\hline 372 & 16.58 & 1.87 & 14.83 & 14.14 & 13.84 & M5 & 0.00 & 0.00 & 3.49 & -1.01 & 0.17 & 6.58 & 0.91 \\
\hline 373 & 17.25 & 2.32 & 14.16 & 12.82 & 12.08 & - & - & 6.87 & - & - & - & - & 2.65 \\
\hline 374 & 12.94 & 0.45 & 12.29 & 11.84 & 11.79 & K5 & 0.00 & 0.00 & 3.64 & 0.23 & 1.12 & 6.27 & - \\
\hline 375 & 13.01 & 0.76 & 11.93 & 11.28 & 11.11 & K5 & 0.58 & 0.00 & 3.64 & 0.34 & 1.12 & 6.12 & 2.50 \\
\hline 376 & - & - & - & - & - & - & - & - & - & - & - & - & - \\
\hline 377 & 15.39 & 1.48 & 13.92 & 13.21 & 13.01 & M5 & 0.00 & 0.00 & 3.49 & -0.54 & 0.22 & 6.19 & 1.18 \\
\hline 378 & - & - & - & - & - & - & - & - & - & - & - & - & - \\
\hline 379 & - & - & - & - & - & - & - & - & - & - & - & - & - \\
\hline 380 & - & - & - & - & - & - & - & - & - & - & - & - & - \\
\hline 381 & 14.95 & 1.17 & 13.54 & 12.90 & 12.64 & - & - & 0.00 & - & - & - & - & 8.53 \\
\hline 382 & 17.36 & 2.14 & 15.25 & 14.72 & 14.43 & - & - & 0.00 & - & - & - & - & - \\
\hline 383 & 13.56 & 0.82 & 12.43 & 11.63 & 11.19 & K6 & 0.54 & 0.00 & 3.62 & 0.10 & 0.91 & 6.25 & - \\
\hline 384 & 15.48 & 1.27 & 14.08 & 13.40 & 13.19 & M2.5 & 0.02 & 0.00 & 3.54 & -0.71 & 0.34 & 6.53 & 8.46 \\
\hline 385 & - & - & - & - & - & - & - & - & - & - & - & - & - \\
\hline 386 & 14.53 & 0.62 & 13.72 & 13.12 & 12.94 & K5 & 0.00 & 1.93 & 3.64 & -0.41 & 0.90 & 7.20 & 1.07 \\
\hline 387 & 16.47 & 1.22 & 15.17 & 14.57 & - & - & - & - & - & - & - & - & 5.08 \\
\hline 388 & 11.69 & 0.46 & 11.11 & 10.70 & 10.64 & - & - & 0.00 & - & - & - & - & - \\
\hline 389 & 15.48 & 1.27 & 13.86 & 13.04 & 12.60 & M1 & 1.12 & 0.00 & 3.57 & -0.47 & 0.47 & 6.45 & - \\
\hline 390 & 17.04 & 1.96 & 15.03 & 14.46 & 14.18 & - & - & 0.00 & - & - & - & - & 0.67 \\
\hline 391 & 15.32 & 1.19 & 13.92 & 13.20 & 13.03 & M1 & 0.76 & 0.00 & 3.57 & -0.49 & 0.48 & 6.50 & 4.30 \\
\hline 392 & 17.04 & 1.68 & 15.35 & 14.72 & 14.71 & - & - & 0.00 & - & - & - & - & 1.89 \\
\hline 393 & - & - & - & - & - & - & - & - & - & - & - & - & - \\
\hline 394 & 10.92 & 0.21 & 10.60 & 10.47 & 10.47 & - & - & 0.00 & - & - & - & - & - \\
\hline 395 & 14.01 & 0.71 & 13.05 & 12.40 & 12.25 & - & - & 0.00 & - & - & - & - & 7.95 \\
\hline 396 & 14.43 & 1.27 & - & 12.27 & - & M5 & 0.00 & - & 3.49 & -0.15 & 0.23 & 5.13 & 0.78 \\
\hline 397 & 14.95 & 1.32 & 13.47 & 12.72 & 12.56 & M3 & 0.00 & 0.00 & 3.53 & -0.49 & 0.32 & 6.26 & - \\
\hline 398 & 12.53 & 0.42 & 12.04 & 11.69 & 11.65 & - & - & 0.00 & - & - & - & - & - \\
\hline 399 & 15.63 & 1.59 & 14.03 & 13.38 & 13.08 & - & - & 0.00 & - & - & - & - & - \\
\hline 400 & 14.33 & 0.92 & 13.06 & 12.39 & 12.10 & M0 & 0.13 & 0.49 & 3.59 & -0.26 & 0.58 & 6.35 & 9.48 \\
\hline 401 & - & - & - & - & - & - & - & - & - & - & - & - & - \\
\hline 402 & 17.07 & 1.71 & 14.83 & 14.18 & 13.81 & - & - & 0.00 & - & - & - & - & - \\
\hline 403 & 15.20 & 1.23 & 13.81 & 13.07 & 12.88 & M1 & 0.94 & 0.55 & 3.57 & -0.40 & 0.48 & 6.38 & 5.08 \\
\hline 404 & 21.05 & - & - & 15.70 & 15.01 & - & - & - & - & - & - & - & - \\
\hline 405 & 13.15 & 0.58 & 12.32 & 11.73 & 11.59 & K2 & 0.49 & 0.00 & 3.69 & 0.29 & 1.54 & 6.79 & 1.54 \\
\hline 406 & 15.72 & 1.72 & 14.02 & 13.35 & 13.15 & M3: & - & 0.00 & - & - & - & - & 0.61 \\
\hline 407 & 16.58 & 1.75 & 14.32 & 13.67 & 13.58 & - & - & 0.00 & - & - & - & - & 3.22 \\
\hline 408 & 12.87 & 0.55 & 12.10 & 11.55 & 11.39 & - & - & 1.61 & - & - & - & - & 5.92 \\
\hline 409 & 15.08 & 1.36 & 13.63 & 12.90 & 12.69 & - & - & 0.47 & - & - & - & - & 1.17 \\
\hline 410 & 15.23 & 1.22 & 13.92 & 13.17 & 12.90 & - & - & 0.88 & - & - & - & - & - \\
\hline 411 & - & - & - & - & - & - & - & - & - & - & - & - & - \\
\hline 412 & 12.19 & 0.52 & 11.41 & 10.98 & 10.85 & G5 & 0.67 & 1.24 & 3.76 & 0.73 & 1.60 & 6.99 & - \\
\hline 413 & 15.06 & 1.02 & 13.78 & 13.09 & 12.90 & M0 & 0.58 & 0.00 & 3.59 & -0.44 & 0.58 & 6.60 & 4.13 \\
\hline 414 & - & - & - & - & - & - & - & - & - & - & - & - & - \\
\hline 415 & - & - & - & - & - & - & - & - & - & - & - & - & - \\
\hline 416 & 15.27 & 1.14 & 13.97 & 13.21 & 13.00 & M2 & 0.00 & 0.69 & 3.55 & -0.65 & 0.38 & 6.52 & 2.79 \\
\hline 417 & 13.26 & 0.74 & 12.27 & 11.63 & 11.47 & K6 & 0.18 & 0.00 & 3.62 & 0.14 & 0.91 & 6.21 & - \\
\hline 418 & 15.13 & 1.05 & 13.71 & 13.00 & 12.81 & M1 & 0.13 & 0.00 & 3.57 & -0.57 & 0.47 & 6.58 & 15.32 \\
\hline 419 & 14.82 & 0.82 & 13.66 & 12.91 & 12.82 & K7 & 0.27 & 0.00 & 3.61 & -0.47 & 0.79 & 6.97 & 6.57 \\
\hline 420 & 12.57 & 0.66 & 11.34 & 10.57 & 10.07 & K4 & 0.71 & 0.00 & 3.66 & 0.57 & 1.59 & 6.12 & 0.88 \\
\hline
\end{tabular}


Table 6. Spectral properties of ACIS sources with more than 50 counts.

\begin{tabular}{|c|c|c|c|c|c|c|c|c|c|c|}
\hline$\overline{\mathrm{N}}$ & $\overline{\overline{\text { Mod }}}$ & $\overline{\overline{n_{H}(\mathrm{Ref})}}$ & $\overline{P P_{\text {null }}}$ & $\begin{array}{c}n_{H} \\
{\left[10^{22} \mathrm{~cm}^{-2}\right]} \\
\end{array}$ & $\begin{array}{c}k T_{1} \\
{[\mathrm{keV}]}\end{array}$ & $\overline{n_{1}}$ & $\begin{array}{c}k T_{2} \\
{[\mathrm{keV}]}\end{array}$ & $\overline{n_{2}}$ & $\begin{array}{c}F_{X}[\mathrm{a}] \\
{\left[\operatorname{ergs} / \mathrm{s} / \mathrm{cm}^{2}\right]}\end{array}$ & $\begin{array}{c}F_{X}[\mathrm{u}] \\
{\left[\mathrm{ergs} / \mathrm{s} / \mathrm{cm}^{2}\right]}\end{array}$ \\
\hline$\overline{2}$ & $\overline{1 \mathrm{~T}}$ & $\overline{\mathrm{Av}}$ & 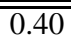 & $\overline{0.00}$ & $0.86_{0.72}^{1.07}$ & $0-5.23_{-5.35}^{-5.14}$ & $\overline{-1-}$ & $\overline{-1-}$ & -14.35 & -14.35 \\
\hline 4 & $1 \mathrm{~T}$ & $\mathrm{Av}$ & 0.62 & 0.10 & $1.29_{1.12}^{1.63}$ & $-4.58_{-4.65}^{-4.53}$ & -- & -- & -13.78 & -13.69 \\
\hline 5 & $2 \mathrm{~T}$ & $\mathrm{Av}$ & 0.14 & 0.26 & $0.26_{0.22}^{0.30}$ & $\begin{array}{l}-3.63_{-3.51}^{-3.59} \\
-\end{array}$ & $1.23_{1.5}^{1.34}$ & $-3.88_{-3.92}^{-3.84}$ & -13.07 & -12.74 \\
\hline 6 & $1 \mathrm{~T}$ & Av & 0.85 & 0.00 & $1.13^{1.39}$ & $-5.00_{-5.99}^{-4.93}$ & -0 & --3.92 & -14.12 & -14.12 \\
\hline 8 & $1 \mathrm{~T}$ & $X$ & 0.41 & $0.62_{0.99}^{0.99}$ & $9.64_{3.36}^{64.00}$ & $-4.67_{-4.79}^{-5.49}$ & -- & -- & -13.62 & -13.48 \\
\hline 9 & $2 \mathrm{~T}$ & Av & 0.92 & 0.09 & $0.31_{0.5}^{0.46}$ & $-4.32^{-4.19}$ & $1.26_{112}^{1.53}$ & $-4.40_{-448}^{-4.34}$ & -13.43 & -13.31 \\
\hline 11 & $1 \mathrm{~T}$ & JHK & 0.17 & 0.10 & $0.98_{0.13}^{1.135}$ & $-4.63_{-477}^{-4.51}$ & -- & --4.48 & -13.86 & -13.75 \\
\hline 13 & $1 \mathrm{~T}$ & X & 0.99 & $1.66_{082}^{2.96}$ & $1.63_{085}^{5.86}$ & $-4.38^{-4.38}$ & -- & -- & -13.98 & -13.45 \\
\hline 14 & $1 \mathrm{~T}$ & Av & 0.51 & 0.00 & $0.80_{063}^{1.85}$ & $-5.30_{-543}^{-5.21}$ & -- & -- & -14.43 & -14.43 \\
\hline 17 & $2 \mathrm{~T}$ & Av & 0.14 & 0.07 & $0.09_{0.02}^{0.63}$ & $-3.48_{-4.5}^{-5.53}$ & $0.95_{080}^{1.21}$ & $-4.93_{-508}^{-4.86}$ & -13.97 & -13.82 \\
\hline 19 & $1 \mathrm{~T}$ & $X$ & 0.37 & $0.00_{0.00}^{0.25}$ & $0.99_{0.74}^{1.20}$ & $-5.20_{-5.31}^{-4.26}$ & -- & -- & -14.31 & -14.31 \\
\hline 25 & $1 \mathrm{~T}$ & Av & 0.48 & 0.14 & $1.52_{113}^{2.09}$ & $\begin{array}{r}-5.31 \\
-4.69_{-475}^{-4.63}\end{array}$ & -- & -- & -13.88 & -13.77 \\
\hline 26 & $2 \mathrm{~T}$ & JHK & 0.19 & 0.16 & $0.38_{024}^{0.13}$ & $\begin{array}{l}-4.75_{-5.08}^{-4.55} \\
-\end{array}$ & $1.36_{100}^{64.00}$ & $-5.02_{-5.34}^{-4.87}$ & -14.01 & -13.80 \\
\hline 27 & $1 \mathrm{~T}$ & $\mathrm{Av}$ & 0.75 & 0.07 & $1.32_{0.95}^{2.24}$ & $\begin{array}{r}-5.08 \\
-5.08_{-5.15}^{-5.00}\end{array}$ & - & -- & -14.25 & -14.18 \\
\hline 29 & $2 \mathrm{~T}$ & $X$ & 0.35 & $0.00_{0.00}^{0.08}$ & $1.09_{0.89}^{1.95}$ & $-4.85_{-5.04}^{-5.18}$ & $64.00_{5.26}^{64.00}$ & $-4.80_{-5.12}^{-4.68}$ & -13.51 & -13.51 \\
\hline 30 & $1 \mathrm{~T}$ & Av & 0.22 & 0.00 & $1.62_{146}^{1.89}$ & $-4.26_{-4.29}^{-4.24}$ & -- & --5.12 & -13.33 & -13.33 \\
\hline 33 & $1 \mathrm{~T}$ & Av & 0.77 & 0.00 & $0.84_{0.05}^{1.05}$ & $-5.10_{-5.49}^{-5.09}$ & -- & -- & -14.22 & -14.22 \\
\hline 36 & $1 \mathrm{~T}$ & Av & 0.71 & 0.02 & $0.79^{1.01}$ & $-4.93^{-4.89}$ & -- & -- & -14.09 & -14.06 \\
\hline 39 & $1 \mathrm{~T}$ & $X$ & 0.23 & $0.00_{0.00}^{0.11}$ & $0.95_{0.76}^{0.69}$ & $-4.68_{-4.53}^{-5.01}$ & -- & -- & -13.80 & -13.80 \\
\hline 45 & $1 \mathrm{~T}$ & $\mathrm{Av}$ & 0.90 & 0.00 & $\begin{array}{r}0.46 \\
1.47_{1.19}^{1.95}\end{array}$ & $-4.96_{-5.94}^{-4.93}$ & -- & -- & -14.05 & -14.05 \\
\hline 52 & $1 \mathrm{~T}$ & $\mathrm{Av}$ & 0.21 & 0.00 & $0.77_{0.66}^{0.86}$ & $-4.86_{-4.93}^{-4.84}$ & -- & -- & -13.99 & -13.99 \\
\hline 53 & $1 \mathrm{~T}$ & $\mathrm{Av}$ & 0.34 & 0.00 & $1.33_{123}^{1.62}$ & $-4.41_{-4.45}^{-4.93}$ & -- & -- & -13.51 & -13.51 \\
\hline 54 & $2 \mathrm{~T}$ & JHK & 0.39 & 0.00 & $0.79_{0.64}^{1.05}$ & $-4.73_{-4.95}^{-4.59}$ & $2.88_{1.96}^{3.84}$ & $-4.49_{-462}^{-4.37}$ & -13.30 & -13.30 \\
\hline 55 & $1 \mathrm{~T}$ & JHK & 0.84 & 0.00 & $1.00_{0.77}^{1.65}$ & $-5.16_{-5.26}^{-5.08}$ & -- & $--^{-4.02}$ & -14.28 & -14.28 \\
\hline 56 & $2 \mathrm{~T}$ & $\mathrm{Av}$ & 0.95 & 0.09 & $0.34_{0.26}^{0.58}$ & $-4.52_{-4.76}^{-4.30}$ & $1.29_{1.03}^{2.00}$ & $-4.81_{-4.91}^{-4.70}$ & -13.74 & -13.61 \\
\hline 59 & $2 \mathrm{~T}$ & $\mathrm{Av}$ & 0.88 & 0.09 & $0.32_{0.26}^{0.41}$ & $-4.38_{-4.52}^{-4.20}$ & $2.90_{1.55}^{64.00}$ & $\begin{array}{l}-4.85_{-5.70}^{-4.71} \\
\end{array}$ & -13.60 & -13.49 \\
\hline 61 & $1 \mathrm{~T}$ & $X$ & 0.24 & $0.00_{0.00}^{0.11}$ & $1.16_{0.96}^{1.360}$ & $-4.92_{-4.99}^{-4.82}$ & -- & ---0.02 & -14.03 & -14.03 \\
\hline 65 & $2 \mathrm{~T}$ & JHK & 0.34 & 0.00 & $1.03_{0.87}^{1.30}$ & $-4.89_{-5.33}^{-4.69}$ & $4.32_{1.99}^{64.00}$ & $-4.91_{-5.31}^{-4.66}$ & -13.58 & -13.58 \\
\hline 68 & $1 \mathrm{~T}$ & $\mathrm{Av}$ & 0.39 & 0.04 & $1.00_{0.81}^{1.49}$ & $-5.31_{-542}^{-5.21}$ & -1.99 & -- & -14.46 & -14.42 \\
\hline 71 & $1 \mathrm{~T}$ & $X$ & 1.00 & $1.93_{143}^{2.57}$ & $6.38_{324}^{23.58}$ & $\begin{array}{l}-4.17^{-3.427} \\
-4.99\end{array}$ & -- & -- & -13.28 & -13.00 \\
\hline 75 & $1 \mathrm{~T}$ & Av & 0.39 & 0.00 & $0.83_{0.70}^{1.24}$ & $\begin{array}{r}-5.17_{-5.29}^{-4.29} \\
-5\end{array}$ & -- & -- & -14.30 & -14.30 \\
\hline 78 & $2 \mathrm{~T}$ & Av & 0.27 & 0.00 & $0.30_{0.23}^{0.70}$ & $\begin{array}{r}-5.27 \\
-4.70_{-5.00}^{-4.53}\end{array}$ & $1.48_{126}^{1.69}$ & $-4.41_{-4.46}^{-4.36}$ & -13.40 & -13.40 \\
\hline 83 & $1 \mathrm{~T}$ & JHK & 0.43 & 0.00 & $2.51_{168}^{5.33}$ & $-5.18^{-5.09}$ & -1.26 & --4.46 & -14.16 & -14.16 \\
\hline 87 & $1 \mathrm{~T}$ & $X$ & 0.47 & $0.00_{0.00}^{0.03}$ & $1.55_{1.38}^{1.71}$ & $-4.34_{-4.37}^{-4.38}$ & -- & -- & -13.42 & -13.42 \\
\hline 89 & $1 \mathrm{~T}$ & JHK & 0.29 & 1.65 & $2.22_{159}^{3.24}$ & $-4.35_{-4.48}^{-4.22}$ & -- & -- & -13.77 & -13.35 \\
\hline 90 & $2 \mathrm{~T}$ & Av & 0.56 & 0.10 & $\begin{array}{r}1.59 \\
0.34_{0.23}^{0.85}\end{array}$ & $\begin{array}{r}-4.41_{-5.03}^{-4.53} \\
-\end{array}$ & $1.26_{101}^{2.07}$ & $-4.77_{-5.58}^{-4.67}$ & -13.80 & -13.67 \\
\hline 91 & $1 \mathrm{~T}$ & Av & 0.28 & 0.27 & $0.70_{0.59}^{0.83}$ & $-4.60_{-4.68}^{-4.03}$ & -- & $--^{-5.58}$ & -14.07 & -13.74 \\
\hline 95 & $1 \mathrm{~T}$ & Av & 0.25 & 0.19 & $1.04_{0.89}^{1.39}$ & $-4.91_{-4.99}^{-4.68}$ & -- & -- & -14.21 & -14.02 \\
\hline 97 & $1 \mathrm{~T}$ & $X$ & 0.97 & $0.22^{0.47}$ & $4.74_{2}^{27.08}$ & $\begin{array}{l}-4.94_{-5.49}^{-4.99} \\
\end{array}$ & -- & -- & -13.90 & -13.81 \\
\hline 98 & $1 \mathrm{~T}$ & $X$ & 0.92 & $2.68_{171}^{4.513}$ & $10.84_{278}^{64.00}$ & $-4.63^{-4.24}$ & -- & -- & -13.72 & -13.43 \\
\hline 102 & $1 \mathrm{~T}$ & Av & 0.91 & 0.09 & $1.04_{0.86}^{1.38}$ & $-4.90_{-5.82}^{-4.86}$ & -- & -- & -14.11 & -14.01 \\
\hline 104 & $2 \mathrm{~T}$ & $\mathrm{Av}$ & 0.32 & 0.00 & $0.46_{0.30}^{0.76}$ & $\begin{array}{l}-5.35_{-5.13}^{-5.00} \\
\end{array}$ & $1.36_{0.01}^{64.00}$ & $-5.49^{-5.27}$ & -14.30 & -14.30 \\
\hline 105 & $1 \mathrm{~T}$ & Av & 0.33 & 0.23 & $0.62_{056}^{0.71}$ & $-4.35^{-5.29}$ & 0.91 & --6.16 & -13.82 & -13.52 \\
\hline 106 & $2 \mathrm{~T}$ & $\mathrm{Av}$ & 0.10 & 0.00 & $0.94_{0.74}^{1.38}$ & $\begin{array}{l}-5.52_{-5.25}^{-4.43} \\
-5\end{array}$ & $64.00_{4.71}^{64.00}$ & $-5.27_{-5.73}^{-5.09}$ & -14.03 & -14.03 \\
\hline 107 & $1 \mathrm{~T}$ & X & 0.27 & $2.86_{2.18}^{4.54}$ & $64.00_{3.93}^{64.00}$ & $-4.51_{-466}^{-4.42}$ & -- & --5 & -13.64 & -13.40 \\
\hline 108 & $1 \mathrm{~T}$ & $\mathrm{X}$ & 0.76 & $2.80_{189}^{4.184}$ & $2.56^{6.68}$ & $-4.35^{-4.62}$ & -- & -- & -13.81 & -13.33 \\
\hline 111 & $2 \mathrm{~T}$ & $\mathrm{Av}$ & 0.15 & $0.12^{189}$ & $0.12_{0.01}^{0.20}$ & $-3.76_{-4.51}^{-1.65}$ & $0.87_{0.83}^{0.97}$ & $-4.48_{-455}^{-4.44}$ & -13.62 & -13.40 \\
\hline 112 & $2 \mathrm{~T}$ & $\mathrm{Av}$ & 0.21 & 0.03 & $0.76_{0.65}^{0.81}$ & $-4.92_{-509}^{-4.81}$ & $4.96_{1.42}^{64.00}$ & $\begin{array}{r}-5.45_{-5.84}^{-5.03} \\
-.03\end{array}$ & -13.89 & -13.86 \\
\hline 114 & $2 \mathrm{~T}$ & JHK & 0.91 & 0.00 & $0.72_{0.59}^{0.83}$ & $\begin{array}{l}-4.70_{-4.89}^{-4.57} \\
\end{array}$ & $2.06_{1.69}^{2.47}$ & $-4.40_{-4.51}^{-4.31}$ & -13.28 & -13.28 \\
\hline 119 & $1 \mathrm{~T}$ & $\mathrm{Av}$ & 0.80 & 0.20 & $1.00_{0.85}^{1.27}$ & $-4.86_{-4.94}^{-4.99}$ & -- & $--^{-4}$ & -14.17 & -13.97 \\
\hline 120 & $2 \mathrm{~T}$ & $\mathrm{Av}$ & 0.23 & 0.12 & $0.28_{0.21}^{0.40}$ & $-4.48_{-4.72}^{-4.34}$ & $1.26_{1.09}^{1.87}$ & $-4.56_{-4.61}^{-4.49}$ & -13.64 & -13.48 \\
\hline 124 & $2 \mathrm{~T}$ & $\mathrm{Av}$ & 0.49 & 0.00 & $0.42_{0.26}^{0.66}$ & $-5.09_{-5.47}^{-4.84}$ & $32.94_{975}^{64.00}$ & $-4.53_{-4.61}^{-4.41}$ & -13.33 & -13.33 \\
\hline 127 & $1 \mathrm{~T}$ & $X$ & 0.56 & $0.90_{0.59}^{1.30}$ & $3.46_{2.00}^{10.11}$ & $\begin{array}{l}-4.47_{-4.26}^{-4.41} \\
-\end{array}$ & -- & $---^{-4}$ & -13.63 & -13.39 \\
\hline 133 & $1 \mathrm{~T}$ & $X$ & 0.23 & $0.00_{0.00}^{0.06}$ & $1.44_{1.13}^{1.73}$ & $\begin{array}{l}-4.82_{-4.78}^{-4.06} \\
-7\end{array}$ & -- & -- & -13.91 & -13.91 \\
\hline 135 & $2 \mathrm{~T}$ & Av & 0.12 & 0.11 & $0.81_{0.73}^{0.96}$ & $\begin{array}{r}-4.37_{-4.29}^{-4.88} \\
\end{array}$ & $3.74_{2}^{7.28}$ & $-4.48_{-461}^{-4.34}$ & -13.22 & -13.13 \\
\hline 136 & $1 \mathrm{~T}$ & Av & 0.07 & 0.29 & $1.81_{151}^{2.39}$ & $\begin{array}{r}-4.77_{-4.81}^{-4.49} \\
\end{array}$ & -2.5 & --4 & -14.01 & -13.82 \\
\hline 141 & $1 \mathrm{~T}$ & $X$ & 0.72 & $2.62_{1.51}^{4.34}$ & $2.04_{1.01}^{6.51}$ & $-4.42^{-4.86}$ & -- & -- & -13.99 & -13.44 \\
\hline 142 & $1 \mathrm{~T}$ & $\mathrm{Av}$ & 0.78 & 0.21 & $1.24_{109}^{1.49}$ & $-4.32_{-4.37}^{-4.27}$ & -- & -- & -13.60 & -13.42 \\
\hline 149 & $2 \mathrm{~T}$ & JHK & 0.38 & 0.00 & $1.90_{117}^{2.59}$ & $-3.86_{-445}^{-3.78}$ & $34.45_{912}^{64.00}$ & $-3.96_{-410}^{-3.85}$ & -12.55 & -12.55 \\
\hline 151 & $1 \mathrm{~T}$ & $\mathrm{Av}$ & 0.80 & 0.04 & $1.98_{1.72}^{2.17}$ & $\begin{array}{r}-4.45 \\
-4.19_{-4.23}^{-4.16}\end{array}$ & -- & --4.10 & -13.25 & -13.22 \\
\hline
\end{tabular}


Table 6. (Continued)

\begin{tabular}{|c|c|c|c|c|c|c|c|c|c|c|}
\hline$\overline{\mathrm{N}}$ & Mod & $\overline{n_{H}(\text { Ref })}$ & $\overline{\bar{P} P_{\text {null }}}$ & $\begin{array}{c}n_{H} \\
{\left[10^{22} \mathrm{~cm}^{-2}\right]} \\
\end{array}$ & $\begin{array}{c}k T_{1} \\
{[\mathrm{keV}]}\end{array}$ & $\overline{\overline{n_{1}}}$ & $\begin{array}{c}k T_{2} \\
{[\mathrm{keV}]}\end{array}$ & $\overline{\overline{n_{2}}}$ & $\begin{array}{c}F_{X}[\mathrm{a}] \\
{\left[\mathrm{ergs} / \mathrm{s} / \mathrm{cm}^{2}\right]}\end{array}$ & $\begin{array}{c}F_{X}[\mathrm{u}] \\
{\left[\mathrm{ergs} / \mathrm{s} / \mathrm{cm}^{2}\right]}\end{array}$ \\
\hline$\overline{\overline{154}}$ & $\overline{2 \mathrm{~T}}$ & $\overline{\mathrm{Av}}$ & 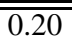 & 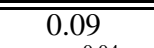 & $\overline{0.75_{0.60}^{0.91}}$ & $=0.57_{-4.74}^{-4.47}$ & $64.00_{1.99}^{64.00}$ & $\begin{array}{l}-4.71_{-5.12}^{-4.53} \\
\end{array}$ & $\overline{-13.41}$ & -13.35 \\
\hline 155 & $1 \mathrm{~T}$ & $\mathrm{X}$ & 0.56 & $0.00_{0.00}^{0.04}$ & $1.48_{123}^{1.80}$ & $-4.77_{-4.83}^{-4.74}$ & -- & $--^{-5.12}$ & -13.85 & -13.85 \\
\hline 161 & $1 \mathrm{~T}$ & JHK & 0.48 & 2.37 & $8.35_{454}^{34.16}$ & $-4.48_{-4.39}^{-4.39}$ & -- & -- & -13.58 & -13.29 \\
\hline 162 & $1 \mathrm{~T}$ & $X$ & 0.58 & $2.73_{161}^{4.43}$ & $1.27^{3.63}$ & $-4.29^{-4.31}$ & -- & -- & -14.19 & -13.39 \\
\hline 163 & $1 \mathrm{~T}$ & JHK & 0.48 & 0.30 & $1.80_{143}^{2.38}$ & $-4.60^{-4.54}$ & -- & -- & -13.84 & -13.65 \\
\hline 165 & $1 \mathrm{~T}$ & $X$ & 0.73 & $3.02_{2.09}^{4.30}$ & $2.09_{1.11}^{1.65}$ & $-4.14^{-3.01}$ & -- & -- & -13.72 & -13.15 \\
\hline 167 & $2 \mathrm{~T}$ & $\mathrm{Av}$ & 0.49 & 0.30 & $0.97_{0.77}^{1.21}$ & $-4.28_{-453}^{-4.16}$ & $3.48_{248}^{6.19}$ & $\begin{array}{l}-4.17_{-4.39}^{-4.03} \\
\end{array}$ & -13.08 & -12.91 \\
\hline 172 & $2 \mathrm{~T}$ & $\mathrm{Av}$ & 0.45 & 0.05 & $0.95_{083}^{0.04}$ & $\begin{array}{l}-4.29_{-4.49}^{-4.33} \\
-\end{array}$ & $3.57_{2}^{6.078}$ & $-4.35_{-4.21}^{-4.29}$ & -13.06 & -13.02 \\
\hline 176 & $1 \mathrm{~T}$ & $X$ & 0.33 & $0.23^{0.29}$ & $2.54_{2.24}^{3.24}$ & $-4.03_{-4.4}^{-3.94}$ & --.56 & --4.51 & -13.13 & -13.00 \\
\hline 178 & $1 \mathrm{~T}$ & X & 0.85 & $0.35_{0.12}^{0.74}$ & $10.84_{3.01}^{64.00}$ & $-4.96_{-5.07}^{-4.19}$ & -- & -- & -13.85 & -13.75 \\
\hline 179 & $2 \mathrm{~T}$ & JHK & 0.82 & $0.13^{12}$ & $0.97_{0.80}^{1.20}$ & $-4.50_{-475}^{-4.36}$ & $4.47_{2.84}^{7.49}$ & $-4.39_{-453}^{-4.25}$ & -13.19 & -13.10 \\
\hline 181 & $1 \mathrm{~T}$ & $\mathrm{X}$ & 0.64 & $0.00_{000}^{0.11}$ & $1.01_{087}^{1.26}$ & $-4.68_{-4.75}^{-4.57}$ & -- & -- & -13.80 & -13.80 \\
\hline 182 & $1 \mathrm{~T}$ & X & 0.89 & $2.97_{171}^{4.60}$ & $1.11_{059}^{2.36}$ & $-4.09^{-4.24}$ & -- & -- & -14.14 & -13.20 \\
\hline 183 & $2 \mathrm{~T}$ & $X$ & 0.31 & $0.04_{0.00}^{0.61}$ & $0.14_{0.02}^{0.24}$ & $-4.08_{-484}^{-2.70}$ & $0.90_{0.20}^{7.42}$ & $-4.93_{-5.13}^{-3.72}$ & -13.79 & -13.70 \\
\hline 184 & $2 \mathrm{~T}$ & Av & 0.32 & 0.04 & $0.74_{0.61}^{0.85}$ & $-4.46_{-468}^{-4.33}$ & $1.85_{112}^{6.100}$ & $-4.68_{-513}^{-4.47}$ & -13.40 & -13.35 \\
\hline 185 & $2 \mathrm{~T}$ & Av & 0.40 & 0.08 & $0.31_{023}^{0.42}$ & $-4.68_{-499}^{-4.59}$ & $1.28_{1.07}^{1.48}$ & $-4.81_{-499}^{-4.71}$ & -13.80 & -13.70 \\
\hline 187 & $2 \mathrm{Tab}^{a}$ & $\mathrm{X}$ & 0.09 & $0.00_{0.00}^{0.12}$ & $0.64_{0.51}^{0.78}$ & $-5.50_{-5.91}^{-5.91}$ & $1.69_{1.31}^{2.78}$ & $-4.92_{-5.33}^{-4.68}$ & -13.62 & -13.62 \\
\hline 189 & $1 \mathrm{~T}$ & X & 0.41 & $0.19_{0.06}^{0.00}$ & $0.67_{0.77}^{0.79}$ & $-4.58_{-482}^{-4.36}$ & -- & $--^{-0.33}$ & -13.94 & -13.70 \\
\hline 190 & $1 \mathrm{~T}$ & $X$ & 0.35 & $0.00_{0.00}^{0.03}$ & $1.30_{1.21}^{1.44}$ & $-4.27_{-4.30}^{-4.23}$ & -- & -- & -13.37 & -13.37 \\
\hline 192 & $1 \mathrm{~T}$ & $X$ & 0.61 & $2.17_{1.48}^{0.00}$ & $1.51_{1.08}^{2.36}$ & $-3.55^{-3.20}$ & -- & -- & -13.26 & -12.63 \\
\hline 193 & $2 \mathrm{~T}$ & Av & 0.85 & 0.06 & $0.76_{0.63}^{0.085}$ & $-4.36_{-451}^{-4.26}$ & $3.34_{2.33}^{5.83}$ & $-4.28_{-441}^{-4.17}$ & -13.07 & -13.02 \\
\hline 194 & $1 \mathrm{~T}$ & $X$ & 0.92 & $2.57_{1.69}^{3.69}$ & $2.39_{152}^{4.759}$ & $-3.74^{-3.39}$ & $--\frac{2.35}{4}$ & $--^{-4.41}$ & -13.21 & -12.72 \\
\hline 196 & $1 \mathrm{~T}$ & JHK & 0.95 & $0.00^{2}$ & $1.22_{090}^{1.826}$ & $-5.17^{-5.09}$ & -- & -- & -14.24 & -14.24 \\
\hline 197 & $1 \mathrm{~T}$ & $\mathrm{Av}$ & 0.18 & 0.49 & $10.07_{-10.04}^{64.00}$ & $-5.09_{-5.27}^{-5.87}$ & -- & -- & -14.02 & -13.89 \\
\hline 199 & $1 \mathrm{~T}$ & JHK & 0.59 & 3.17 & $2.04^{2.68}$ & $-3.83_{-3.96}^{-5.23}$ & -- & -- & -13.44 & -12.85 \\
\hline 200 & $1 \mathrm{~T}$ & $\mathrm{Av}$ & 0.32 & 0.21 & $1.23^{1.63}$ & $-4.78_{-481}^{-4.96}$ & -- & -- & -14.07 & -13.89 \\
\hline 202 & $1 \mathrm{~T}$ & $\mathrm{Av}$ & 0.87 & 0.22 & $2.57_{168}^{6.100}$ & $-4.96_{-508}^{-4.86}$ & -- & -- & -14.05 & -13.93 \\
\hline 203 & $1 \mathrm{~T}$ & $\mathrm{Av}$ & 0.25 & 0.00 & $2.46_{1.32}^{5.08}$ & $\begin{array}{r}-5.08 \\
-5.07_{-517}^{-4.98}\end{array}$ & -- & -- & -14.05 & -14.05 \\
\hline 204 & $1 \mathrm{~T}$ & JHK & 0.74 & 0.00 & $1.46_{139}^{1.32}$ & $\begin{array}{r}-5.17 \\
-4.70_{-4.76}^{-4.66}\end{array}$ & -- & -- & -13.79 & -13.79 \\
\hline 205 & $1 \mathrm{~T}$ & JHK & 0.71 & 0.00 & $5.43_{2.09}^{64.00}$ & $\begin{array}{l}-5.40_{-5.51}^{-5.38} \\
\end{array}$ & -- & -- & -14.24 & -14.24 \\
\hline 206 & $2 \mathrm{~T}$ & $\mathrm{Av}$ & 0.41 & 0.04 & $0.64_{0.55}^{0.76}$ & $-4.31_{-4.46}^{-4.31}$ & $1.44_{1.32}^{1.58}$ & $-3.87_{-3.93}^{-3.82}$ & -12.89 & -12.84 \\
\hline 207 & $1 \mathrm{~T}$ & JHK & 0.75 & 0.23 & $0.68_{058}^{0.85}$ & $-4.67_{-4.78}^{-4.56}$ & -- & $--^{-3.93}$ & -14.11 & -13.82 \\
\hline 208 & $1 \mathrm{~T}$ & $X$ & 0.85 & $1.18_{0.66}^{1.75}$ & $0.68_{0.41}^{1.28}$ & $-4.42^{-3.78}$ & -- & -- & -14.49 & -13.56 \\
\hline 212 & $2 \mathrm{~T}$ & $\mathrm{Av}$ & 0.28 & $0.24^{0.66}$ & $0.19^{0.262}$ & $-4.12_{-455}^{-3.90}$ & $0.95_{0.77}^{1.36}$ & $-4.65_{-482}^{-4.48}$ & -13.85 & -13.45 \\
\hline 216 & $2 \mathrm{~T}$ & $\mathrm{Av}$ & 0.98 & 0.00 & $0.64_{0.26}^{0.78}$ & $-4.87_{-4.97}^{-4.35}$ & $1.70_{1.22}^{2.60}$ & $-4.97_{-5.29}^{-4.80}$ & -13.73 & -13.73 \\
\hline 226 & $1 \mathrm{~T}$ & JHK & 0.81 & 0.00 & $0.98_{0.79}^{1.50}$ & $\begin{array}{r}-5.13_{-5.22}^{-5.91} \\
\end{array}$ & -- & $--^{-5.29}$ & -14.24 & -14.24 \\
\hline 227 & $1 \mathrm{~T}$ & $\mathrm{Av}$ & 0.95 & 0.04 & $1.65_{131}^{2.41}$ & $-4.99_{-507}^{-4.92}$ & -- & -- & -14.08 & -14.05 \\
\hline 228 & $1 \mathrm{~T}$ & $X$ & 0.42 & $6.18_{4.48}^{8.14}$ & $4.64_{2.63}^{27.03}$ & $-3.86_{-4.10}^{-3.56}$ & -- & -- & -13.24 & -12.72 \\
\hline 229 & $2 \mathrm{~T}$ & $\mathrm{Av}$ & 0.13 & 0.06 & $0.43_{0.31}^{0.538}$ & $-4.61_{-4.82}^{-4.40}$ & $1.13_{1.01}^{1.28}$ & $-4.51_{-4.61}^{-4.44}$ & -13.52 & -13.44 \\
\hline 231 & $1 \mathrm{~T}$ & $\mathrm{Av}$ & 0.35 & 0.08 & $1.25^{1.46}$ & $-4.75_{-4.61}^{-4.82}$ & -- & $--^{-4}$ & -13.93 & -13.85 \\
\hline 234 & $2 \mathrm{Tab}^{b}$ & $X$ & 0.02 & $0.06_{0.02}^{0.08}$ & $0.80_{066}^{1.60}$ & $-4.86_{-5.14}^{-4.51}$ & $2.98_{2.64}^{3.34}$ & $-3.76_{-3.82}^{-3.70}$ & -12.64 & -12.60 \\
\hline 235 & $1 \mathrm{~T}$ & $\mathrm{X}$ & 0.45 & $4.79^{7.4 .42}$ & $1.99_{1.10}^{6.68}$ & $-3.98^{-3.35}$ & -- & --3.82 & -13.71 & -13.00 \\
\hline 238 & $1 \mathrm{~T}$ & $\mathrm{Av}$ & 0.35 & $0.11^{2.96}$ & $1.53^{1.97}$ & $-4.66_{-471}^{-4.61}$ & -- & -- & -13.83 & -13.74 \\
\hline 240 & $2 \mathrm{~T}$ & $\mathrm{Av}$ & 0.23 & 0.24 & $0.24^{0.30}$ & $\begin{array}{r}-3.69_{-3.87}^{-3.51} \\
-\end{array}$ & $1.64_{1.37}^{2.40}$ & $-4.43_{-4.54}^{-4.35}$ & -13.39 & -13.01 \\
\hline 241 & $1 \mathrm{~T}$ & X & 0.17 & $0.33_{0.00}^{0.65}$ & $0.60_{0.38}^{0.84}$ & $-4.95^{-4.34}$ & -- & -- & -14.54 & -14.12 \\
\hline 242 & $1 \mathrm{~T}$ & JHK & 0.48 & 3.98 & $1.33_{111}^{1.68}$ & $-3.61_{-375}^{-3.48}$ & -- & -- & -13.60 & -12.71 \\
\hline 244 & $1 \mathrm{~T}$ & $X$ & 0.76 & $2.22^{2.59}$ & $4.61_{3.19}^{7.117}$ & $-3.71_{-3.81}^{-3.60}$ & -- & -- & -12.90 & -12.57 \\
\hline 245 & $1 \mathrm{~T}$ & Av & 0.87 & 0.09 & $0.73_{0.61}^{0.85}$ & $-4.89_{-4.98}^{-4.81}$ & -- & -- & -14.15 & -14.03 \\
\hline 246 & $1 \mathrm{~T}$ & $X$ & 0.86 & $0.17^{0.45}$ & $1.56_{113}^{2.89}$ & $-4.70_{-4.82}^{-4.58}$ & -- & -- & -13.91 & -13.78 \\
\hline 248 & $1 \mathrm{~T}$ & $\mathrm{X}$ & 0.94 & $2.30_{159}^{3.356}$ & $1.82_{1.70}^{2.73}$ & $-4.20^{-3.77}$ & -- & -- & -13.80 & -13.24 \\
\hline 249 & $2 \mathrm{~T}$ & Av & 0.75 & 0.08 & $1.03_{091}^{1.111}$ & $-4.10_{-426}^{-3.86}$ & $3.80_{2.86}^{13.95}$ & $-3.99_{-42.89}^{-3.89}$ & -12.77 & -12.72 \\
\hline 250 & $1 \mathrm{~T}$ & Av & 0.44 & 0.00 & $1.26^{2.110}$ & $-5.15^{-5.07}$ & -- & --4.29 & -14.26 & -14.26 \\
\hline 252 & $1 \mathrm{~T}$ & Av & 0.49 & 0.21 & $1.01^{1.29}$ & $-4.88^{-5.25}$ & -- & -- & -14.20 & -13.99 \\
\hline 254 & $1 \mathrm{~T}$ & Av & 0.65 & 0.02 & $1.12^{1.40}$ & $-5.21^{-5.12}$ & -- & -- & -14.35 & -14.32 \\
\hline 255 & $1 \mathrm{~T}$ & $X$ & 0.89 & $0.95^{1.11}$ & $5.57^{7.89}$ & $\begin{array}{r}-5.32 \\
-3.67^{-3.60}\end{array}$ & -- & -- & -12.71 & -12.51 \\
\hline 257 & $1 \mathrm{~T}$ & $\mathrm{X}$ & 0.02 & $3.82^{4.79}$ & $4.82^{40.94}$ & $\begin{array}{r}-3.72 \\
-3.89^{-3.65}\end{array}$ & - & - & -13.16 & -12.75 \\
\hline 260 & $1 \mathrm{~T}$ & JHK & 0.35 & $\begin{array}{c}0.023 .15 \\
0.81\end{array}$ & $4.21_{2.76 .23}^{16.23}$ & $-5.01_{-51}^{-4.85}$ & -- & -- & -14.10 & -13.89 \\
\hline 261 & $2 \mathrm{~T}$ & JHK & 0.67 & 0.00 & $0.94_{0.83}^{1.03}$ & $-4.23_{-4.38}^{-4.11}$ & $2.75_{2.12}^{4.18}$ & $-4.21_{-4.36}^{-4.09}$ & -12.94 & -12.94 \\
\hline 263 & $1 \mathrm{~T}$ & $\mathrm{Av}$ & 0.25 & 0.21 & $1.30_{1.04}^{3.83}$ & $-5.13_{-5.25}^{-5.38}$ & -2.1 & --4 & -14.41 & -14.24 \\
\hline 265 & $1 \mathrm{~T}$ & Av & 0.44 & 0.00 & $1.24_{1.35}^{1.34}$ & $-4.35_{-4.39}^{-4.32}$ & -- & -- & -13.46 & -13.46 \\
\hline
\end{tabular}


Table 6. (Continued)

\begin{tabular}{|c|c|c|c|c|c|c|c|c|c|c|}
\hline$\overline{\mathrm{N}}$ & $\overline{\text { Mod }}$ & $\overline{n_{H}(\mathrm{Ref})}$ & $\overline{P P_{\text {null }}}$ & $\begin{array}{c}n_{H} \\
{\left[10^{22} \mathrm{~cm}^{-2}\right]} \\
\end{array}$ & $\begin{array}{c}k T_{1} \\
{[\mathrm{keV}]} \\
\end{array}$ & $\overline{n_{1}}$ & $\begin{array}{c}k T_{2} \\
{[\mathrm{keV}]} \\
\end{array}$ & $\overline{n_{2}}$ & $\begin{array}{c}F_{X}[\mathrm{a}] \\
{\left[\mathrm{ergs} / \mathrm{s} / \mathrm{cm}^{2}\right]} \\
\end{array}$ & $\begin{array}{c}F_{X}[\mathrm{u}] \\
{\left[\operatorname{ergs} / \mathrm{s} / \mathrm{cm}^{2}\right]}\end{array}$ \\
\hline 268 & $\overline{1 \mathrm{~T}}$ & $\overline{\text { JHK }}$ & 0.38 & $\overline{0.00}$ & $0.79_{0.58}^{1.07}$ & $-5.15_{-5.24}^{-5.07}$ & 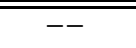 & ב-- & -14.28 & -14.28 \\
\hline 269 & $1 \mathrm{~T}$ & Av & 0.60 & 0.24 & $0.97_{0.90}^{1.04}$ & $-4.22_{-4.26}^{-4.18}$ & -- & -- & -13.58 & -13.34 \\
\hline 273 & $1 \mathrm{~T}$ & JHK & 0.87 & 1.25 & $1.32_{1.04}^{1.77}$ & $-4.50_{-4.63}^{-4.48}$ & -- & -- & -14.15 & -13.60 \\
\hline 274 & $1 \mathrm{~T}$ & $X$ & 0.94 & $8.92_{7.33}^{11.55}$ & $63.98_{-63.96}^{64.00}$ & $\begin{array}{l}-3.79_{-3.89}^{-3.09} \\
\end{array}$ & -- & -- & -13.10 & -12.68 \\
\hline 275 & $1 \mathrm{~T}$ & $\mathrm{Av}$ & 0.12 & 0.04 & $1.62_{1.26}^{2.29}$ & $-5.26_{-5.39}^{-5.16}$ & -- & -- & -14.36 & -14.33 \\
\hline 276 & $1 \mathrm{~T}$ & $\mathrm{Av}$ & 0.91 & 0.02 & $1.23_{1.12}^{1.39}$ & $-4.50_{-4.55}^{-4.46}$ & -- & -- & -13.63 & -13.61 \\
\hline 278 & $2 \mathrm{~T}$ & $\mathrm{Av}$ & 0.78 & 0.14 & $0.24_{0.18}^{0.34}$ & $-4.35_{-4.60}^{-4.14}$ & $1.29_{1.07}^{1.68}$ & $-4.78_{-4.90}^{-4.69}$ & -13.79 & -13.56 \\
\hline 279 & $1 \mathrm{~T}$ & $\mathrm{X}$ & 0.63 & $2.66_{1.62}^{4.36}$ & $2.02_{0.98}^{5.59}$ & $-4.41^{-3.75}$ & -- & -- & -13.99 & -13.43 \\
\hline 280 & $1 \mathrm{~T}$ & JHK & 0.15 & $1.33^{2.02}$ & $2.24_{178}^{2.89}$ & $-4.10_{-41}^{-4.02}$ & -- & -- & -13.49 & -13.11 \\
\hline 282 & $1 \mathrm{~T}$ & JHK & 0.66 & 0.83 & $3.63_{2.84}^{4.70}$ & $-4.17_{-4.23}^{-4.11}$ & -- & -- & -13.31 & -13.07 \\
\hline 285 & $1 \mathrm{~T}$ & JHK & 0.68 & 0.00 & $1.45_{0.97}^{2.45}$ & $-5.32_{-5.44}^{-5.23}$ & -- & -- & -14.41 & -14.41 \\
\hline 287 & $1 \mathrm{~T}$ & $X$ & 0.71 & $2.79_{1.52}^{4.55}$ & $1.13_{0.58}^{2.76}$ & $-3.99^{-3.08}$ & -- & -- & -14.00 & -13.10 \\
\hline 290 & $1 \mathrm{~T}$ & Av & 0.40 & 0.00 & $1.87_{1.54}^{2.29}$ & $-4.44_{-449}^{-4.40}$ & -- & -- & -13.49 & -13.49 \\
\hline 292 & $1 \mathrm{~T}$ & JHK & 0.74 & 0.80 & $2.13_{1.57}^{3.34}$ & $-4.63_{-4.76}^{-4.59}$ & -- & -- & -13.95 & -13.64 \\
\hline 293 & $1 \mathrm{~T}$ & $\mathrm{Av}$ & 0.90 & 0.21 & $2.13_{1.44}^{5.18}$ & $\begin{array}{l}-5.14_{-5.28}^{-5.60} \\
-\end{array}$ & -- & -- & -14.28 & -14.15 \\
\hline 294 & $1 \mathrm{~T}$ & Av & 0.62 & 0.00 & $0.96_{0.74}^{1.91}$ & $-5.40_{-5.52}^{-5.30}$ & -- & -- & -14.52 & -14.52 \\
\hline 296 & $1 \mathrm{~T}$ & $X$ & 0.87 & $1.92_{1.55}^{2.36}$ & $4.80_{3.02}^{9.62}$ & $-3.94_{-4.07}^{-3.78}$ & -- & -- & -13.11 & -12.80 \\
\hline 297 & $1 \mathrm{~T}$ & JHK & 0.95 & 0.00 & $0.39_{0.32}^{0.56}$ & $-4.89_{-5.12}^{-4.76}$ & -- & -- & -14.22 & -14.22 \\
\hline 300 & $2 \mathrm{Tab}^{c}$ & $\mathrm{Av}$ & 0.10 & 0.19 & $0.32_{0.26}^{0.40}$ & $-4.67_{-5.05}^{-4.32}$ & $1.46_{1.27}^{1.68}$ & $-4.47_{-4.58}^{-4.28}$ & -13.40 & -13.20 \\
\hline 301 & $1 \mathrm{~T}$ & $X$ & 0.74 & $0.00_{0.00}^{0.13}$ & $0.74_{0.62}^{0.80}$ & $-4.82_{-4.88}^{-4.03}$ & -- & $--^{-4.50}$ & -13.95 & -13.95 \\
\hline 302 & $2 \mathrm{~T}$ & JHK & 0.80 & 0.00 & $0.81_{0.72}^{0.94}$ & $-4.16_{-4.30}^{-3.98}$ & $1.61_{1.46}^{1.73}$ & $-3.82_{-3.98}^{-3.76}$ & -12.75 & -12.75 \\
\hline 305 & $1 \mathrm{~T}$ & $\mathrm{X}$ & 0.65 & $4.84_{326}^{8.95}$ & $64.00_{3.94}^{64.00}$ & $-4.64_{-4.44}^{-4.49}$ & $--^{1.40}$ & $--^{-3.98}$ & -13.82 & -13.51 \\
\hline 307 & $1 \mathrm{~T}$ & Av & 0.87 & $0.01^{5.20}$ & $0.95_{0.77}^{1.24}$ & $-5.36_{-5.48}^{-5.26}$ & -- & -- & -14.48 & -14.47 \\
\hline 311 & $1 \mathrm{~T}$ & $\mathrm{Av}$ & 0.33 & 0.35 & $3.03_{2,14}^{4: 76}$ & $-4.67_{-4.76}^{-3.68}$ & -- & -- & -13.77 & -13.61 \\
\hline 318 & $1 \mathrm{~T}$ & $X$ & 0.57 & $0.00_{0.00}^{0.04}$ & $2.35_{1.84}^{3.31}$ & $-4.60_{-4.66}^{-4.56}$ & -- & -- & -13.59 & -13.59 \\
\hline 324 & $1 \mathrm{~T}$ & $X$ & 0.44 & $3.49_{2.50}^{5.50}$ & $21.64_{2.97}^{64.00}$ & $-4.53_{-4.64}^{-4.15}$ & -- & -- & -13.59 & -13.30 \\
\hline 326 & $1 \mathrm{~T}$ & $X$ & 0.78 & $0.00_{0.00}^{2.79}$ & $0.94_{0.23}^{1.15}$ & $\begin{array}{l}-5.21_{-5.31}^{-3.64} \\
\end{array}$ & -- & -- & -14.33 & -14.33 \\
\hline 327 & $1 \mathrm{~T}$ & $\mathrm{X}$ & 0.38 & $5.78_{4.63}^{0.00}$ & $3.22_{2.20}^{6.23}$ & $\begin{array}{r}-5.31 \\
-3.53_{-3.78}^{-3.29}\end{array}$ & -- & -- & -13.00 & -12.42 \\
\hline 328 & $2 \mathrm{~T}$ & $\mathrm{X}$ & 0.41 & $0.00_{0.00}^{4.68}$ & $0.95_{0.57}^{1.18}$ & $-4.81_{-5.17}^{-4.61}$ & $10.85_{2.12}^{64.00}$ & $-4.94_{-5.23}^{-4.63}$ & -13.44 & -13.44 \\
\hline 329 & $1 \mathrm{~T}$ & $\mathrm{X}$ & 0.32 & $0.15_{0.08}^{0.21}$ & $0.74_{0.64}^{0.79}$ & $-4.03_{-4.12}^{-3.88}$ & --.12 & $--^{-5.23}$ & -13.27 & -13.08 \\
\hline 332 & $1 \mathrm{~T}$ & $X$ & 0.28 & $0.05_{0.02}^{0.08}$ & $1.87_{1.55}^{2.04}$ & $-3.86_{-3.90}^{-3.82}$ & -- & -- & -12.94 & -12.90 \\
\hline 333 & $1 \mathrm{~T}$ & $\mathrm{Av}$ & 0.37 & 0.04 & $1.30_{1.11}^{1.75}$ & $-4.84_{-4.91}^{-3.70}$ & -- & -- & -13.98 & -13.94 \\
\hline 334 & $1 \mathrm{~T}$ & $X$ & 0.40 & $0.00_{0.00}^{0.09}$ & $1.15_{0.97}^{1.31}$ & $-4.87_{-4.94}^{-4.80}$ & -- & -- & -13.98 & -13.98 \\
\hline 338 & $2 \mathrm{~T}$ & $\mathrm{Av}$ & 0.63 & 0.00 & $0.99_{0.84}^{1.117}$ & $-4.78_{-5.06}^{-4.94}$ & $5.12_{2.34}^{31.58}$ & $-4.97_{-5.22}^{-4.72}$ & -13.56 & -13.56 \\
\hline 340 & $1 \mathrm{~T}$ & $X$ & 0.21 & $0.00_{0.00}^{0.03}$ & $1.47_{1.29}^{1.64}$ & $-4.30_{-4.33}^{-4.06}$ & -- & $--^{-5.22}$ & -13.38 & -13.38 \\
\hline 341 & $2 \mathrm{~T}$ & JHK & 0.44 & 0.00 & $0.89_{0.78}^{1.09}$ & $-4.70_{-4.98}^{-4.48}$ & $2.19_{1.67}^{3.84}$ & $-4.47_{-4.72}^{-4.35}$ & -13.31 & -13.31 \\
\hline 343 & $1 \mathrm{~T}$ & $\mathrm{Av}$ & 0.87 & 0.09 & $1.74_{1,54}^{2: 18}$ & $-4.48_{-4.53}^{-4.48}$ & -- & $--^{-4.12}$ & -13.61 & -13.54 \\
\hline 344 & $1 \mathrm{~T}$ & $\mathrm{Av}$ & 0.25 & 0.12 & $6.21_{2.99}^{26.94}$ & $-4.99_{-5.07}^{-4.91}$ & -- & -- & -13.85 & -13.80 \\
\hline 345 & $1 \mathrm{~T}$ & $\mathrm{Av}$ & 0.89 & 0.02 & $0.93_{0.70}^{1.1 .12}$ & $-5.16_{-5.26}^{-5.08}$ & -- & -- & -14.30 & -14.28 \\
\hline 348 & $1 \mathrm{~T}$ & $X$ & 0.31 & $0.11_{0.04}^{0.22}$ & $1.47_{1.27}^{1: 71}$ & $-4.43_{-4.51}^{-4.39}$ & -- & -- & -13.60 & -13.50 \\
\hline 350 & $2 \mathrm{~T}$ & $\mathrm{Av}$ & 0.48 & 0.11 & $0.80_{0.70}^{0.98}$ & $-4.10_{-4.25}^{-3.98}$ & $1.64_{1.49}^{1.90}$ & $-3.75_{-3.90}^{-3.68}$ & -12.78 & -12.67 \\
\hline 352 & $1 \mathrm{~T}$ & $X$ & 0.46 & $0.02_{0.00}^{0.10}$ & $1.91_{1.53}^{2.51}$ & $-4.51_{-4.57}^{-4.43}$ & --- & -- & -13.56 & -13.55 \\
\hline 353 & $2 \mathrm{~T}$ & $\mathrm{Av}$ & 0.10 & 0.14 & $0.27_{0.19}^{0.39}$ & $-4.66_{-5.00}^{-4.39}$ & $3.00_{2.09}^{4.76}$ & $-4.79_{-4.90}^{-4.69}$ & -13.71 & -13.58 \\
\hline 354 & $1 \mathrm{~T}$ & $\mathrm{Av}$ & 0.41 & 0.00 & $1.66_{1.41}^{2.07}$ & $-4.68_{-4.73}^{-4.60}$ & --.09 & $--^{-4.9}$ & -13.75 & -13.75 \\
\hline 359 & $2 \mathrm{~T}$ & $\mathrm{Av}$ & 0.24 & 0.00 & $0.40_{0.05}^{0.61}$ & $-5.24_{-608}^{-4.85}$ & $1.50_{133}^{1.84}$ & $-4.61_{-466}^{-4.54}$ & -13.64 & -13.64 \\
\hline 360 & $1 \mathrm{~T}$ & $\mathrm{Av}$ & 0.37 & 0.00 & $1.32_{1.18}^{1.68}$ & $-4.05_{-4.10}^{-4.01}$ & -- & -- & -13.16 & -13.16 \\
\hline 361 & $1 \mathrm{~T}$ & $\mathrm{Av}$ & 0.32 & 0.25 & $1.97_{0.91}^{7.59}$ & $-5.15_{-5.35}^{-5.02}$ & -- & -- & -14.34 & -14.18 \\
\hline 362 & $1 \mathrm{~T}$ & $X$ & 0.25 & $0.00_{0.00}^{0.09}$ & $1.24_{1.02}^{1.58}$ & $-5.02_{-5.10}^{-4.93}$ & -- & -- & -14.12 & -14.12 \\
\hline 364 & $1 \mathrm{~T}$ & $\mathrm{Av}$ & 0.60 & $0.13^{\circ}$ & $0.81_{0.74}^{0.91}$ & $-4.49_{-4.55}^{-4.45}$ & -- & -- & -13.78 & -13.62 \\
\hline 365 & $2 \mathrm{~T}$ & $\mathrm{Av}$ & 0.25 & 0.33 & $0.80_{0.26}^{0.99}$ & $-4.89_{-5.07}^{-4.77}$ & $64.00_{2.54}^{64.00}$ & $-5.35_{-5.92}^{-5.06}$ & -14.04 & -13.81 \\
\hline 366 & $1 \mathrm{~T}$ & $X$ & 0.74 & $4.15_{2.71}^{6.44}$ & $2.00_{1.10}^{4.99}$ & $-4.16^{-3.56}$ & -- & $--^{-5}$ & -13.85 & -13.19 \\
\hline 367 & $2 \mathrm{~T}$ & $\mathrm{Av}$ & 0.18 & 0.00 & $0.29_{0.18}^{0.73}$ & $-5.05_{-553}^{-4.85}$ & $1.54_{105}^{12.24}$ & $-5.23_{-5.53}^{-5.10}$ & -14.09 & -14.09 \\
\hline 368 & $1 \mathrm{~T}$ & JHK & 0.37 & 0.00 & $0.97_{0.78}^{1.27}$ & $-4.93_{-500}^{-5.85}$ & --1.05 & $--^{-5}$ & -14.05 & -14.05 \\
\hline 370 & $2 \mathrm{~T}$ & $\mathrm{Av}$ & 0.17 & 0.00 & $0.65_{0.25}^{0.38}$ & $\begin{array}{r}-5.00 \\
-5.41_{-5.99}^{-5.19}\end{array}$ & $8.38_{1.10}^{64.00}$ & $-5.24_{-5.47}^{-5.04}$ & -13.94 & -13.94 \\
\hline 375 & $1 \mathrm{~T}$ & $X$ & 0.24 & $0.00_{0.00}^{0.02}$ & $\begin{array}{l}1.30_{1.24}^{1.325} \\
0\end{array}$ & $\begin{array}{r}-5.99 \\
-4.03_{-4.05}^{-4.00}\end{array}$ & -- & --5.47 & -13.10 & -13.10 \\
\hline 381 & $1 \mathrm{~T}$ & JHK & 0.77 & 0.00 & $1.17_{1.03}^{1.34}$ & $\begin{array}{r}-4.75_{-4.80}^{-4.65} \\
-\end{array}$ & -- & -- & -13.85 & -13.85 \\
\hline 383 & $2 \mathrm{~T}$ & $\mathrm{Av}$ & 0.34 & 0.09 & $0.40_{0.28}^{0.93}$ & $-4.44_{-4.68}^{-4.80}$ & $1.55_{1.18}^{5.22}$ & $-4.48_{-4.94}^{-4.37}$ & -13.44 & -13.34 \\
\hline 384 & $1 \mathrm{~T}$ & Av & 0.27 & 0.00 & $0.99_{0.80}^{1.38}$ & $-4.96_{-5.03}^{-4.90}$ & -- & --4 & -14.08 & -14.07 \\
\hline 386 & $1 \mathrm{~T}$ & $\mathrm{Av}$ & 0.22 & 0.00 & $1.52_{1.11}^{2.00}$ & $-4.94_{-501}^{-4.85}$ & -- & -- & -13.97 & -13.97 \\
\hline 388 & $2 \mathrm{~T}$ & JHK & 0.10 & 0.00 & $0.99_{0.85}^{1.06}$ & $-4.14_{-432}^{-4.06}$ & $2.72_{1.77}^{3.81}$ & $\begin{array}{l}-4.44_{-469}^{-4.19}\end{array}$ & -13.02 & -13.02 \\
\hline
\end{tabular}


Table 6. (Continued)

\begin{tabular}{|c|c|c|c|c|c|c|c|c|c|c|}
\hline$\overline{\mathrm{N}}$ & $\overline{\mathrm{Mod}}$ & $\overline{n_{H}(\mathrm{Ref})}$ & 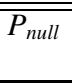 & $\begin{array}{c}n_{H} \\
{\left[10^{22} \mathrm{~cm}^{-2}\right]}\end{array}$ & $\begin{array}{c}k T_{1} \\
{[\mathrm{keV}]}\end{array}$ & $n_{1}$ & $\begin{array}{c}k T_{2} \\
{[\mathrm{keV}]}\end{array}$ & $n_{2}$ & $\begin{array}{c}F_{X}[\mathrm{a}] \\
{\left[\mathrm{ergs} / \mathrm{s} / \mathrm{cm}^{2}\right]}\end{array}$ & $\begin{array}{c}F_{X}[\mathrm{u}] \\
{\left[\mathrm{ergs} / \mathrm{s} / \mathrm{cm}^{2}\right]}\end{array}$ \\
\hline$\overline{389}$ & $\overline{\overline{1 T}}$ & $\overline{\overline{\mathrm{Av}}}$ & 0.95 & $\overline{0.18}$ & $6.84_{3.62}^{25.05}$ & $\begin{array}{l}-5.02_{-5.95}^{-4.95} \\
\end{array}$ & 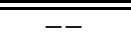 & $\overline{-1-}$ & $\overline{-13.91}$ & $\overline{-13.84}$ \\
\hline 391 & $1 \mathrm{~T}$ & $X$ & 0.26 & $0.00^{0.03}$ & $1.92^{2.50}$ & $-4.57^{-4.52}$ & -- & -- & -13.60 & -13.60 \\
\hline 395 & $1 \mathrm{~T}$ & JHK & 0.79 & 0.00 & $1.25^{1.38}$ & $-4.51_{-456}^{-4.42}$ & -- & -- & -13.62 & -13.62 \\
\hline 396 & $1 \mathrm{~T}$ & Av & 0.50 & 0.00 & $1.29_{1.58}^{1.58}$ & $-4.69_{-475}^{-4.64}$ & -- & -- & -13.79 & -13.79 \\
\hline 397 & $1 \mathrm{~T}$ & Av & 0.52 & 0.00 & $1.03^{1.29}$ & $-5.01_{-5.95}^{-4.95}$ & -- & -- & -14.13 & -14.13 \\
\hline 400 & $1 \mathrm{~T}$ & Av & 0.27 & 0.02 & $1.09_{0.90}^{1.31}$ & $\begin{array}{l}-5.20_{-5}^{-5.10} \\
-11\end{array}$ & -- & -- & -14.34 & -14.31 \\
\hline 403 & $1 \mathrm{~T}$ & Av & 0.90 & 0.15 & $0.46_{032}^{0.964}$ & $\begin{array}{r}-5.99^{-4.75} \\
-525\end{array}$ & -- & -- & -14.49 & -14.25 \\
\hline 405 & $1 \mathrm{~T}$ & Av & 0.41 & 0.08 & $1.33_{1.26}^{1.52}$ & $-4.10_{-4.13}^{-4.07}$ & -- & -- & -13.28 & -13.20 \\
\hline 407 & $1 \mathrm{~T}$ & JHK & 0.64 & 0.00 & $1.27_{1.03}^{\frac{1.20}{2.07}}$ & $\begin{array}{l}-5.15_{-5.46}^{-5.07} \\
-\end{array}$ & -- & -- & -14.26 & -14.26 \\
\hline 408 & $2 \mathrm{~T}$ & JHK & 0.09 & 0.26 & $0.91_{078}^{1.01}$ & $-4.31_{-4.38}^{-4.26}$ & $64.00_{3}^{64.00}$ & $-4.97_{-5.46}^{-4.75}$ & -13.49 & -13.29 \\
\hline 409 & $1 \mathrm{~T}$ & JHK & 0.28 & 0.08 & $1.00_{076}^{1.38}$ & $-4.84_{-4.92}^{-4.78}$ & -- & $--^{-5.46}$ & -14.04 & -13.96 \\
\hline 411 & $1 \mathrm{~T}$ & $X$ & 0.52 & $0.73_{0.34}^{1.86}$ & $34.26_{2.50}^{64.00}$ & $-4.80_{-4.97}^{-4.92}$ & -- & -- & -13.75 & -13.62 \\
\hline 412 & $1 \mathrm{~T}$ & $\mathrm{Av}$ & 0.71 & 0.11 & $0.96_{0.91}^{1.01}$ & $-4.05_{-4.08}^{-4.03}$ & -- & -- & -13.27 & -13.15 \\
\hline 413 & $1 \mathrm{~T}$ & $\mathrm{Av}$ & 0.82 & 0.09 & $0.98_{081}^{1.91}$ & $-4.91_{-5.80}^{-4.08}$ & -- & -- & -14.13 & -14.03 \\
\hline 414 & $1 \mathrm{~T}$ & $X$ & 0.77 & $1.49_{0.97}^{2.33}$ & $13.66_{3.63}^{64.00}$ & $\begin{array}{r}-59_{-4.30}^{-4.30} \\
-4\end{array}$ & -- & -- & -13.61 & -13.40 \\
\hline 417 & $1 \mathrm{~T}$ & Av & 0.53 & $0.03^{\prime \prime}$ & $1.14_{1.02}^{1.23}$ & $-4.28_{-4.35}^{-4.20}$ & -- & -- & -13.42 & -13.39 \\
\hline 418 & $1 \mathrm{~T}$ & Av & 0.38 & 0.02 & $1.18_{0.99}^{1.02}$ & $\begin{array}{l}-5.19_{-5.31}^{-5.31} \\
-\end{array}$ & -- & -- & -14.32 & -14.30 \\
\hline 419 & $1 \mathrm{~T}$ & $\mathrm{Av}$ & 0.68 & 0.04 & $1.00_{0.84}^{1.20}$ & $-5.03_{-51}^{-4.95}$ & -- & -- & -14.20 & -14.15 \\
\hline 420 & $1 \mathrm{~T}$ & $\mathrm{Av}$ & 0.22 & 0.11 & $1.97_{142}^{3.94}$ & $-4.48_{-4.55}^{-4.42}$ & -- & -- & -13.59 & -13.51 \\
\hline
\end{tabular}

a) Abund. $=1.43_{0.42}^{5.00}$; b) Abund. $=0.84_{0.50}^{1.26}$; c) Abund. $=0.94_{0.38}^{2.69}$; 